\title{
NITI Aayog
}

\section{Analysis of key nutrition indicators based on National Family Health Survey (NFHS)}

\section{Estimates from NFHS 4 (2015-16) and NFHS 5 (2019-2021)}

\author{
Authors: Dr. Neena Bhatia ${ }^{1}$, Komal Rathi' ${ }^{2}$ Charu Arora ${ }^{2}$, Tashi Choedon ${ }^{3}$, Pratik Rajurkar ${ }^{4}$, Dr. Rinky
} Thakur ${ }^{1}$, Vedeika Shekhar ${ }^{1}$, Archa Misra ${ }^{3}$, Surendra Kumar Patel ${ }^{3}$ and Dr. Rakesh Sarwal ${ }^{1}$

${ }^{1}$ NITI Aayog, Government of India, ${ }^{2}$ TATA Trusts, ${ }^{3}$ Institute of Economic Growth, ${ }^{4}$ International Innovation Corps 


\section{Acknowledgement}

This presentation has benefited greatly from the valuable suggestions and feedback of Dr. V.K Paul (Member) Health, NITI Aayog and Mr. Amitabh Kant (CEO, NITI Aayog). The authors are grateful to Institute of Economic Growth (IEG), who prepared the spatial maps used in this slide deck. The authors are thankful to interns Ms. Anjali Rauthan and Ms. Anushka Pandey for their tireless efforts in creating this presentation 


\section{Abstract}

This analysis on key nutrition indicators from successive rounds of the NFHS (4 and 5) is based on all-India and State/UT level factsheet released by Ministry of Health and Family Welfare (MoHFW). The analysis includes:

1. National level trends from NFHS-3 (2005-06), NFHS-4 (2015-16) and NFHS-5 (2019-21) data for 44 indicators including outcomes, service delivery and key determinants of nutrition.

2. State level trends of same indicators- number of states showing improvement / worsening ( \pm 0.1 percent point change), /no change for these indicators, spatial maps based on change in prevalence for these indicators; and charting State performance with respect to achieving Sustainable Development Goals (SDG 2030) targets.

This presentation will be useful for policy makers, State administrators, public health experts, research scholars and research institutes in the area of maternal and child health \& Nutrition. 


\section{Contents}

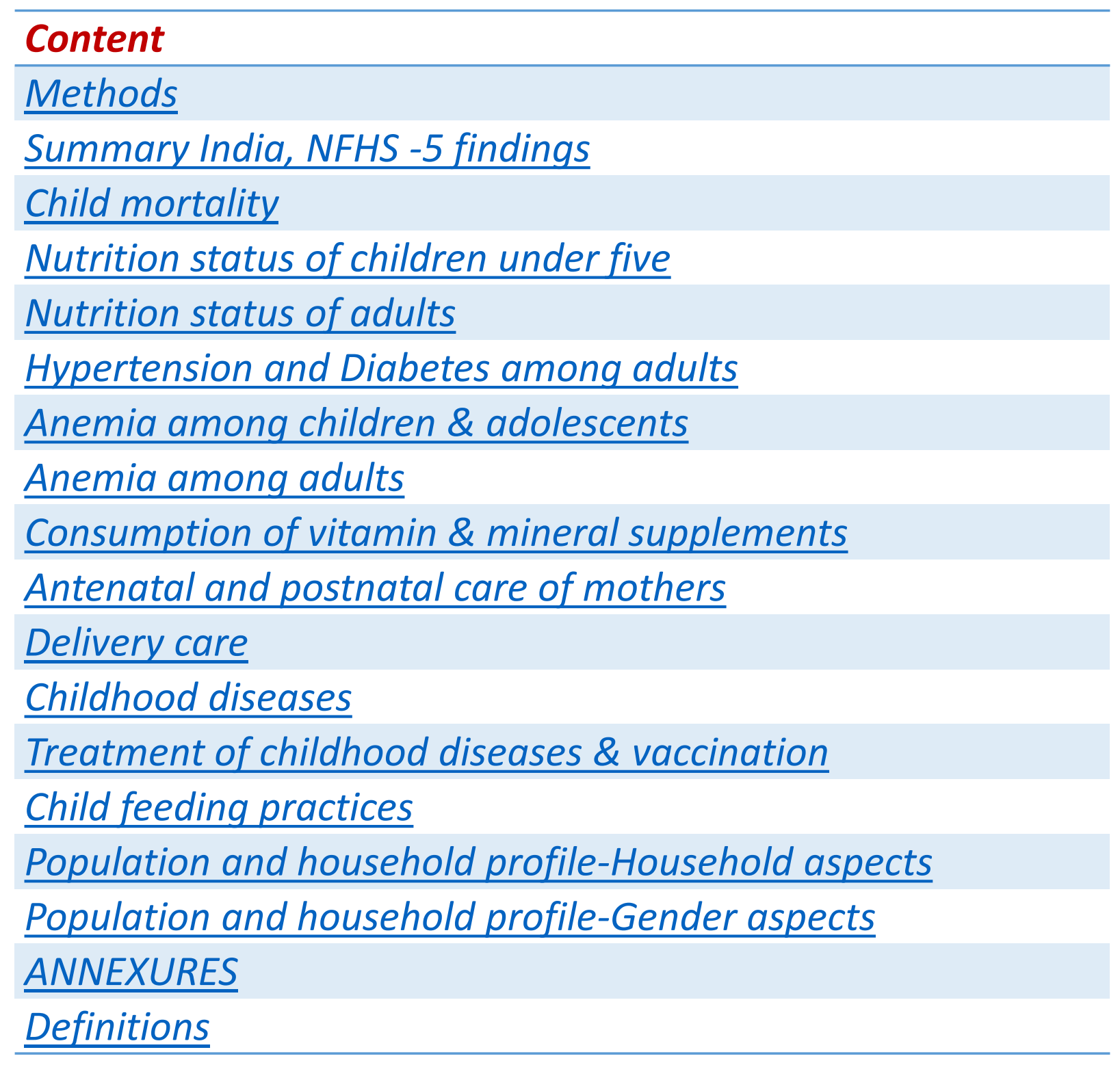




\section{Methods}

Objective: To conduct preliminary, National and State level trend analysis of key nutrition related outcomes, determinants and intervention coverage indicators.

Target audience: Policy and programme officials, Public health experts, Research Scholars \& Research institutes in the area of Maternal and Child health \& Nutrition.

\section{Data Analysis}

Indicators: Select nutrition and health related indicators were analysed (List of indicators and definitions)

Following analysis were done:

1. National level trends from NFHS-3, 2005-06, NFHS-4, 2015-16 and NFHS-5, 2019-21 data for 44 indicators

2. State level trends of these indicators - number of states showing improvement, decline and no change for these indicators based on change percent point ; spatial maps based on change in prevalence for these indicators; State performance chart with respect to achieving Sustainable Development Goals (SDG 2030) targets for these indicators as well as based on change percent point (from NFHS 4 to NFHS 5).

Data sources for trends analysis for India : NFHS-3, 2005-06, NFHS-4, 2015-16 and NFHS-5, 2019-21 factsheets.

$>$ Data sources for State findings: NFHS-4, 2015-16 and NFHS-5, 2019-21 factsheets were used and change in percent point was calculated (NFHS 5- NFHS 4).

$>$ Spatial maps for India for the indicators were prepared by Institute of Economic Growth (IEG), Delhi, using NFHS 5 (2019-21) State factsheets.

$>$ States showing \pm 0.1 percent point change were considered as improving or worsening.

For NFHS 4, only if values for indicators were available in the NFHS 5 factsheets, they were included in the analysis. Back to contents 


\section{Summary of India, NFHS - 5 Findings}

\begin{tabular}{|c|c|}
\hline Indicator & Trend as compared to NFHS-4 \\
\hline \multicolumn{2}{|l|}{ 1. Child Mortality } \\
\hline 1a. Neonatal Mortality & $(-4.5)$ \\
\hline 1b. Infant mortality & $(-5.5)$ \\
\hline 1c. Under 5 mortality & $(-7.8)$ \\
\hline \multicolumn{2}{|c|}{ 2. Nutrition Status of children under 5} \\
\hline 2a. Stunting & $(-2.9 \%)$ \\
\hline 2b. Wasting & $(-1.7 \%)$ \\
\hline 2c. Severe Wasting & $(+0.2 \%)$ \\
\hline 2d. Underweight & $(-3.7 \%)$ \\
\hline 2e. Overweight & $(+1.3 \%)$ \\
\hline \multicolumn{2}{|l|}{ 3. Nutritional Status of adults } \\
\hline 3a. Underweight among women & $(-4.2 \%)$ \\
\hline 3b. Underweight among men & $(-4 \%)$ \\
\hline 3c. Overweight among women & $(+3.4 \%)$ \\
\hline 3d. Overweight among men & $(+4 \%)$ \\
\hline
\end{tabular}

Indicator

Trend as compared to NFHS-4

4. Anemia

4a. Anemia in children (6-59 months)

4b. Anemia in adolescent girls

4c. Anemia in adolescent boys

4d. Anemia in pregnant women

4e. Anemia in all women

4f. Anemia in all men

$(+8.5 \%)$

$(+5 \%)$

$(+1.9 \%)$

$(+1.8 \%)$

$(+3.9 \%)$

$(+2.3 \%)$

5. Consumption of supplements

5a. Iron Folic acid supplement for $100+$ days

5b. Iron Folic acid supplement for 180 days

5c. Vitamin A supplementation among children

$(+11.6 \%)$

6. Antenatal care (ANC) and postnatal care (PNC)

6a. ANC check-up in first trimester

$(+11.4 \%)$

6b. Mothers who received PNC

$(+15.6 \%)$

7. Delivery care

7a. Institutional births

$(+9.7 \%)$

7b. Caesarean delivery 


\section{Summary of India, NFHS - 5 Findings}

\begin{tabular}{|c|c|}
\hline Indicator & $\begin{array}{l}\text { Trend as compared to } \\
\text { NFHS-4 }\end{array}$ \\
\hline \multicolumn{2}{|l|}{ 8. Childhood diseases } \\
\hline 8a. Prevalence of Diarrhoea & $(-1.9 \%)$ \\
\hline 8b. Prevalence of ARI & $(+0.1 \%)$ \\
\hline \multicolumn{2}{|c|}{ 9. Treatment of childhood diseases $\&$ vaccination } \\
\hline $\begin{array}{l}\text { 9a. Children with diarrhoea who } \\
\text { received ORS }\end{array}$ & $(+10 \%)$ \\
\hline $\begin{array}{l}\text { 9b. Children with diarrhoea who } \\
\text { received Zinc }\end{array}$ & $+10.2 \%)$ \\
\hline $\begin{array}{l}\text { 9c. Children with fever or symptoms of } \\
\text { ARI taken to a health facility }\end{array}$ & $(-4.2 \%)$ \\
\hline 9e. Full vaccination & $-14.4 \%)$ \\
\hline \multicolumn{2}{|l|}{ 10. Child feeding practices } \\
\hline $\begin{array}{l}\text { 10a. Breastfeeding within one hour of } \\
\text { birth }\end{array}$ & \\
\hline 10b. Exclusively breastfeeding & $(+8.8 \%)$ \\
\hline 10c. Introduction of semisolids & $(+3.2 \%)$ \\
\hline 10d. Adequate diet among children & $7 \%)$ \\
\hline
\end{tabular}

Indicator

Trend as compared to NFHS-4

11.Population and household profile: household aspect

11a. HHs with improved drinking water $+1.5 \%$

11b. HHs with electricity $+8.8 \%$

11c. HHs with improved sanitation $+21.7$ facility

12. Population and household profile: gender aspect

12a. Sex ratio of total population $+29 \%$

$12 \mathrm{~b}$. Women with 10 or more years of schooling

12c. Women married before 18 years $-3.5 \%$ 


\section{Child Mortality}

\section{A secular decline was observed in neonatal, infant and under-five mortality rates nationally.}

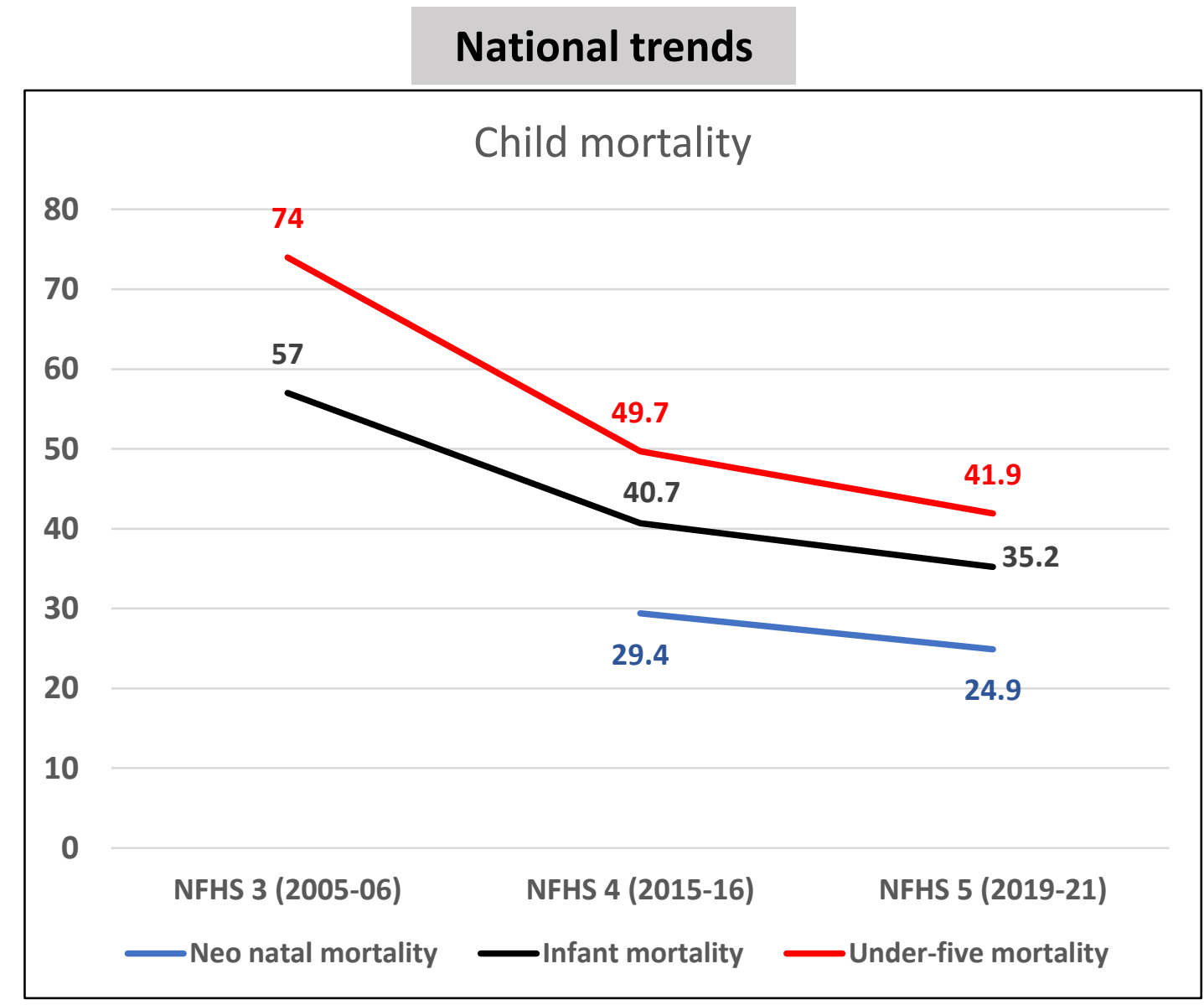

Click here for

State findings

\begin{tabular}{|l|l|l|l|}
\hline $\begin{array}{l}\text { Change over } \\
\text { NFHS 4 }\end{array}$ & $\begin{array}{l}\text { Number } \\
\text { of States } \\
\text { improving }\end{array}$ & $\begin{array}{l}\text { Number of } \\
\text { States with } \\
\text { no change }\end{array}$ & $\begin{array}{l}\text { Number of } \\
\text { States } \\
\text { worsening }\end{array}$ \\
\hline $\begin{array}{l}\text { Neo natal } \\
\text { mortality }\end{array}$ & 26 & 0 & 9 \\
\hline \hline Infant mortality & 30 & 0 & 5 \\
\hline \hline Under-five & 31 & 0 & 4 \\
\hline mortality & & & \\
\hline
\end{tabular}

NFHS 3 for neonatal mortality not available 


\section{Child Mortality}

Change in Prevalence of neonatal mortality rate (NNMR), Infant mortality rate (IMR), and under-five mortality rate (U5MR) in India across NFHS-4\&5 (2015-16 \& 2019-21)

\section{Neonatal mortality rate (NNMR) (-4.5)}

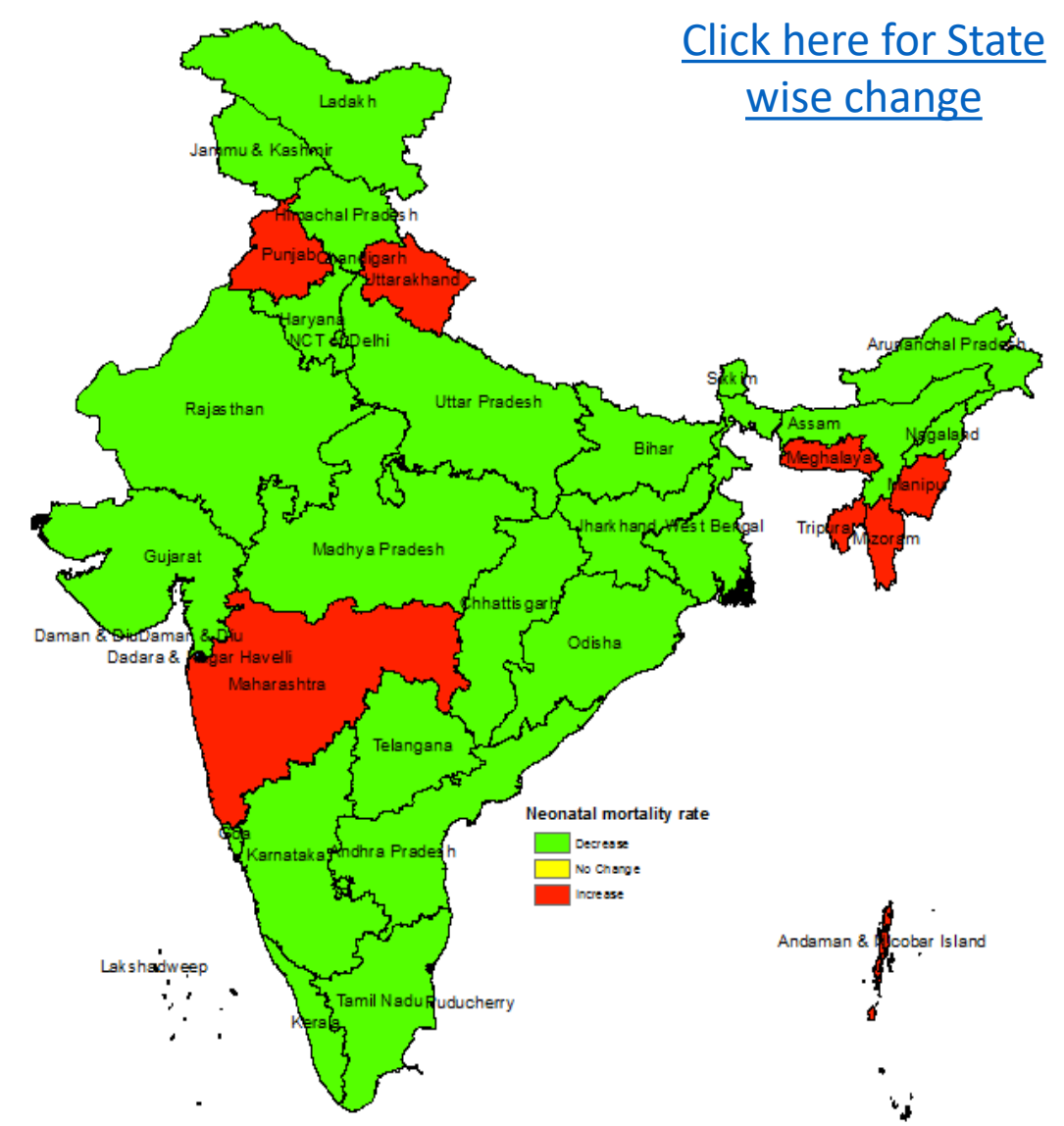

\section{Top 5 states (based on change percent point)}

Lakshadweep (-23.3), Sikkim(-15.8), Ladakh (-14.3), Jammu \& Kashmir (-13.3), Assam (-10.3)

\section{Infant mortality rate (IMR) (-5.5)}

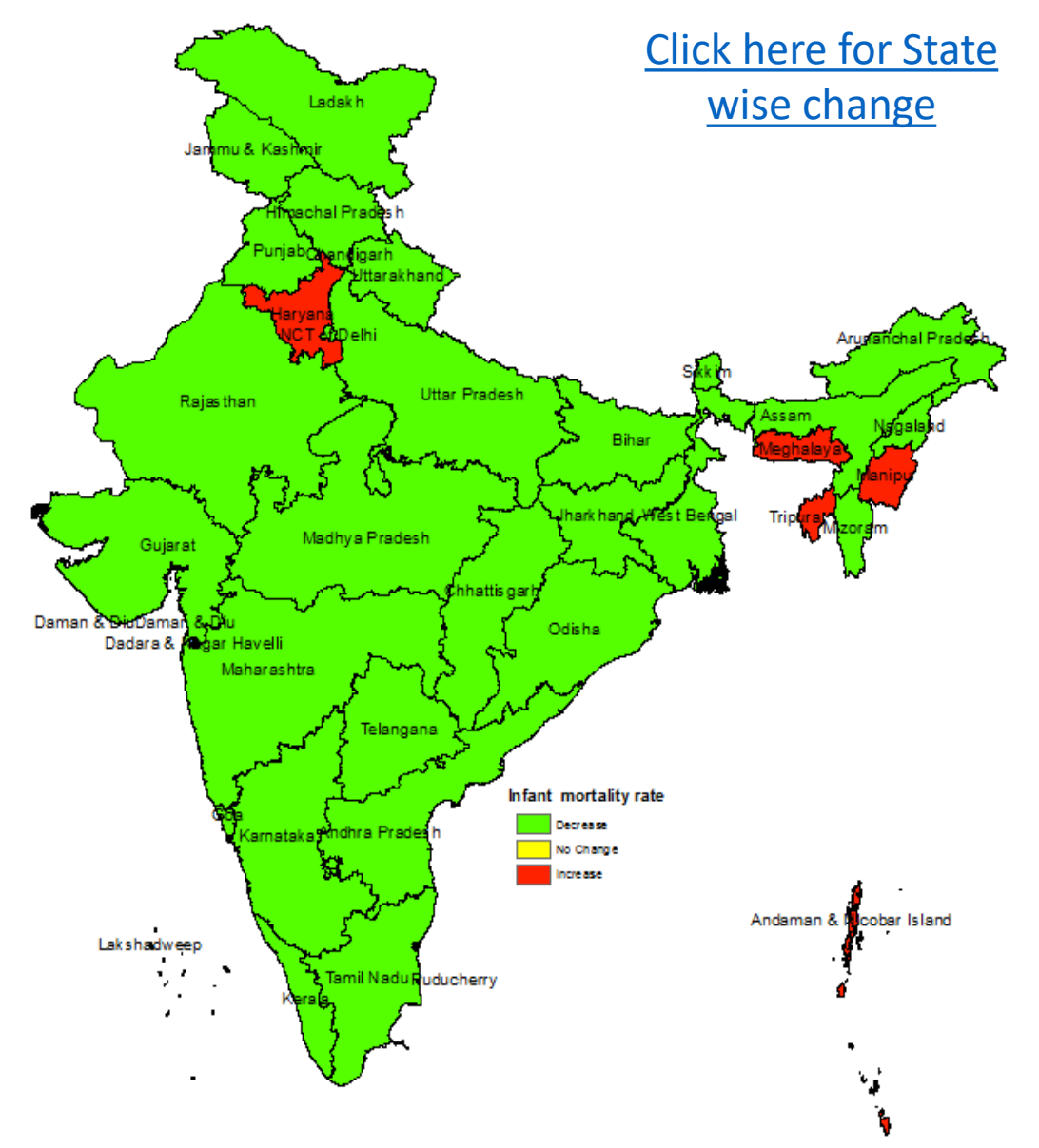

Lakshadweep (-27), Mizoram(-18.8), Sikkim (-18.3), Jammu \& Kashmir(-16.1), Assam(-15.7)

\section{Under-5 mortality rate (U5MR) (-7.8)}

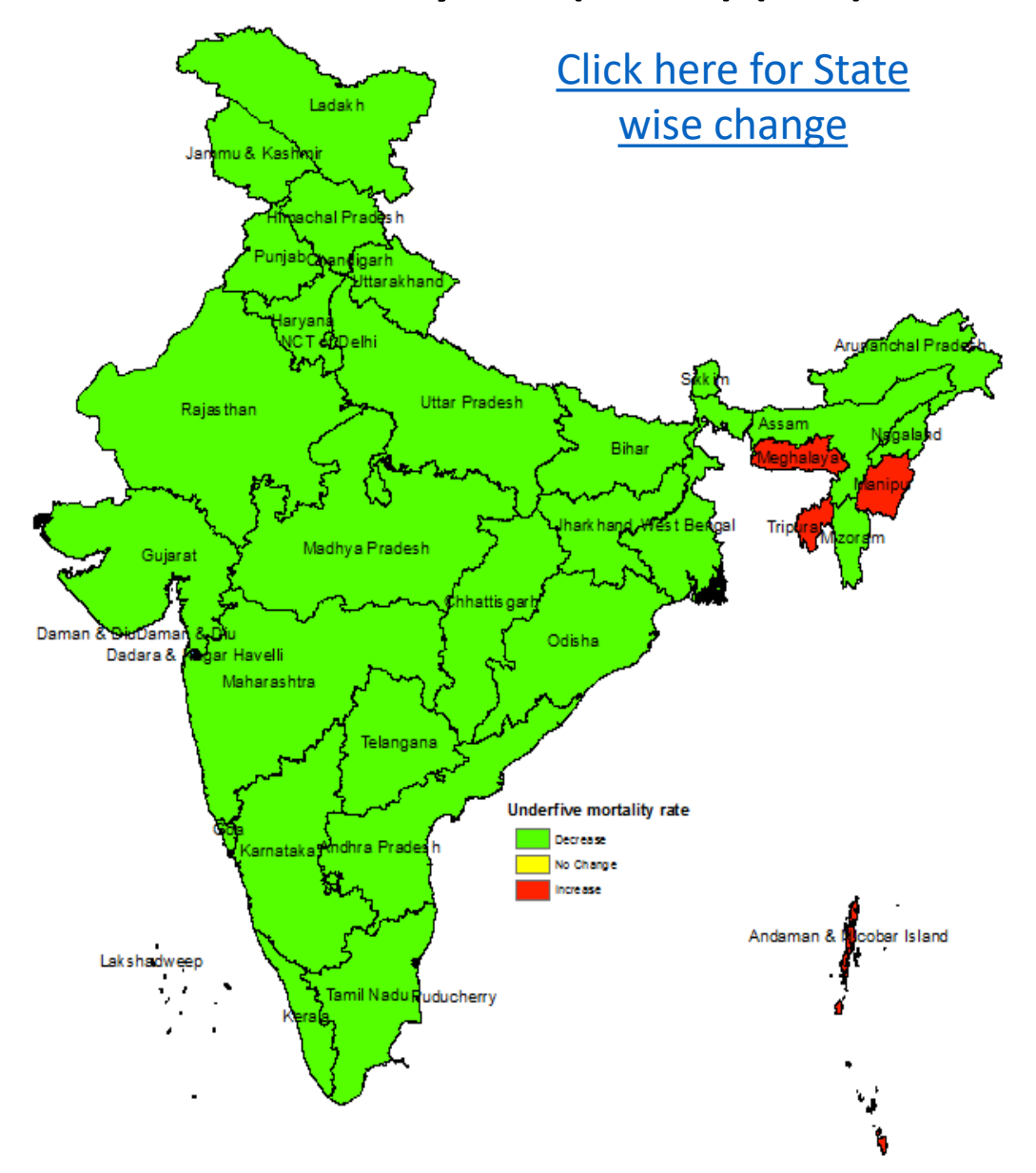

Lakshadweep (30.2), Mizoram (-22), Sikkim(-21), Jammu \& Kashmir(-19.1), Uttar Pradesh(-18.3 ) 
- A secular decline was observed in stunting, wasting and underweight among children nationally

- Severe wasting and overweight has increased

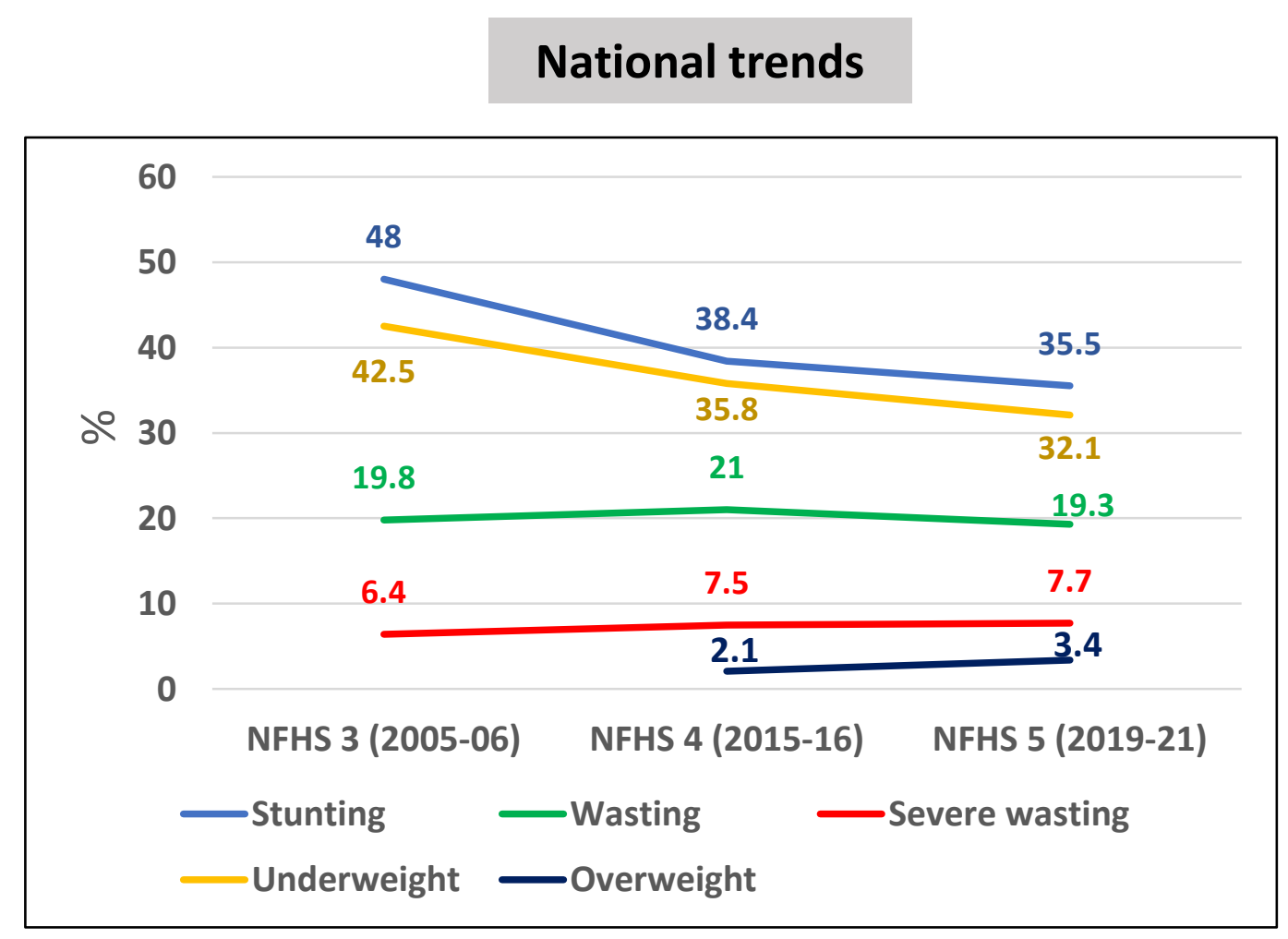

Click here for State findings

\begin{tabular}{|l|l|l|l|}
\hline $\begin{array}{l}\text { Change over } \\
\text { NFHS 4 }\end{array}$ & $\begin{array}{l}\text { Number } \\
\text { of States } \\
\text { improving }\end{array}$ & $\begin{array}{l}\text { Number of } \\
\text { States with } \\
\text { no change }\end{array}$ & $\begin{array}{l}\text { Number of } \\
\text { States } \\
\text { worsening }\end{array}$ \\
\hline Stunting & 23 & 0 & 13 \\
\hline Wasting & 22 & 2 & 12 \\
\hline Severe wasting & 18 & 0 & 18 \\
\hline Underweight & 20 & 0 & 16 \\
\hline Overweight & 3 & 0 & 33 \\
\hline
\end{tabular}

Note: NFHS 3 for overweight not available 


\section{Child Nutritional Status}

Change in prevalence of children under 5 years who are underweight (weight-for-age) overweight (weight-for-height) in India as per NFHS -4 \&5 (2015-16 \& 2019-21)

\section{Underweight children (-3.7\%)}
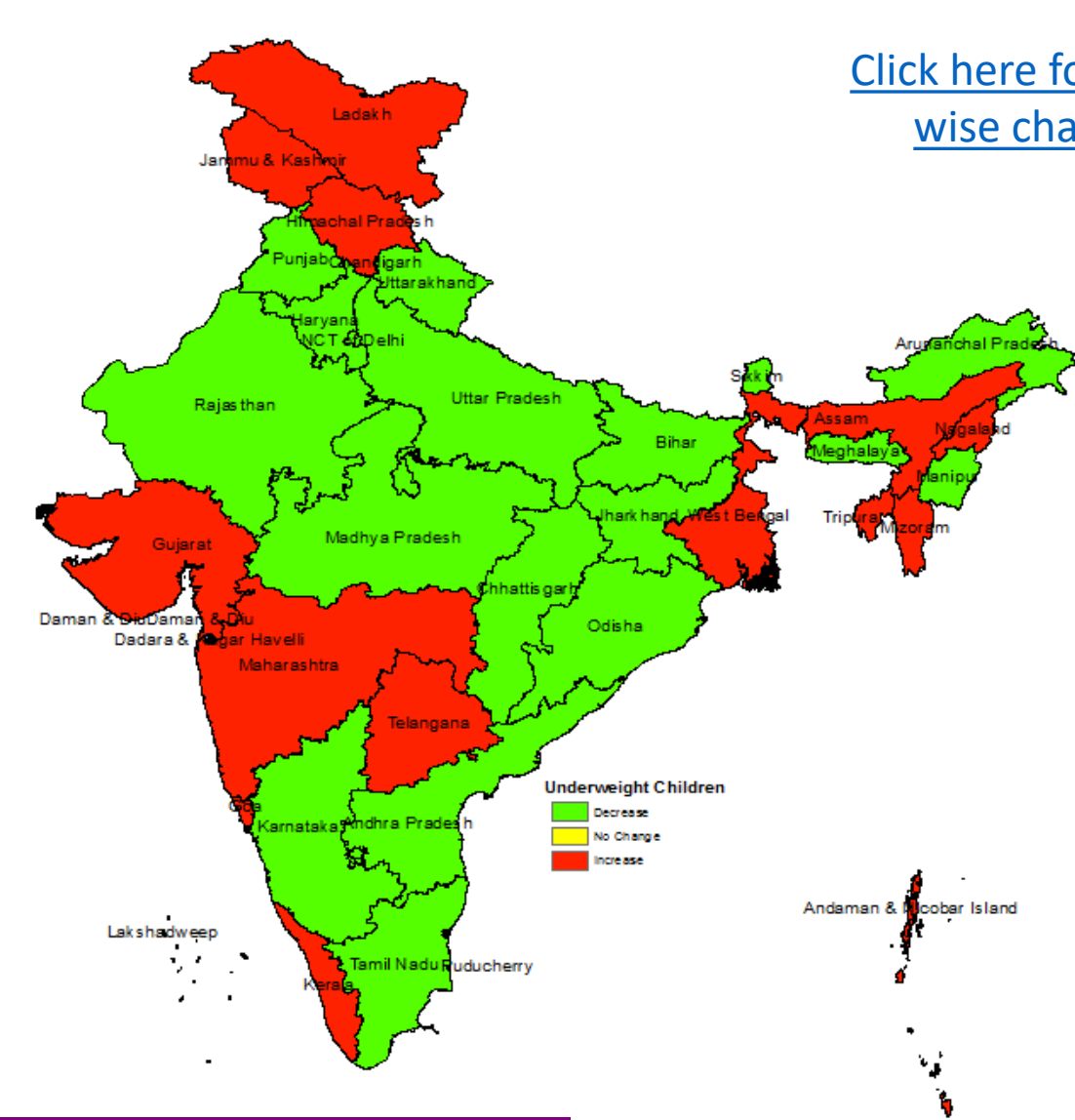

\section{Top 5 states (based on change percent point)}

Madhya Pradesh (-9.8\%), Rajasthan (-9.1\%) Jharkhand (-8.4\%),

Haryana (-7.9\%), Uttar Pradesh (-7.4\%)

\section{Overweight children (+1.3\%)}

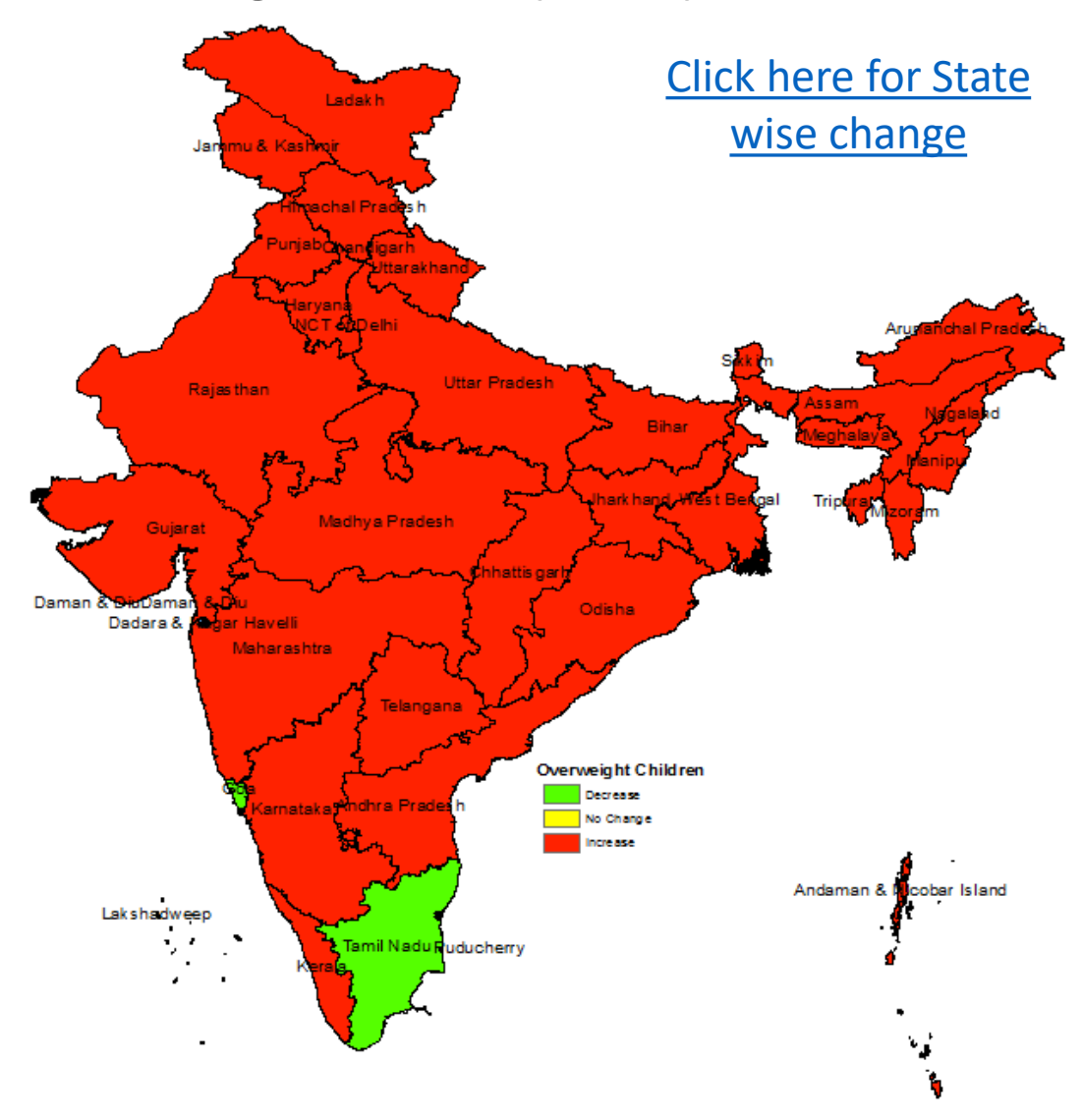

Dadra and Nagar Haveli \&Daman and Diu (-2.0\%), Goa (-0.9\%), Tamil Nadu(-0.7\%) 


\section{Nutritional Status of Adults}

- A secular decline was observed in underweight among adults nationally

- Overweight among adults has increased nationally

National trends

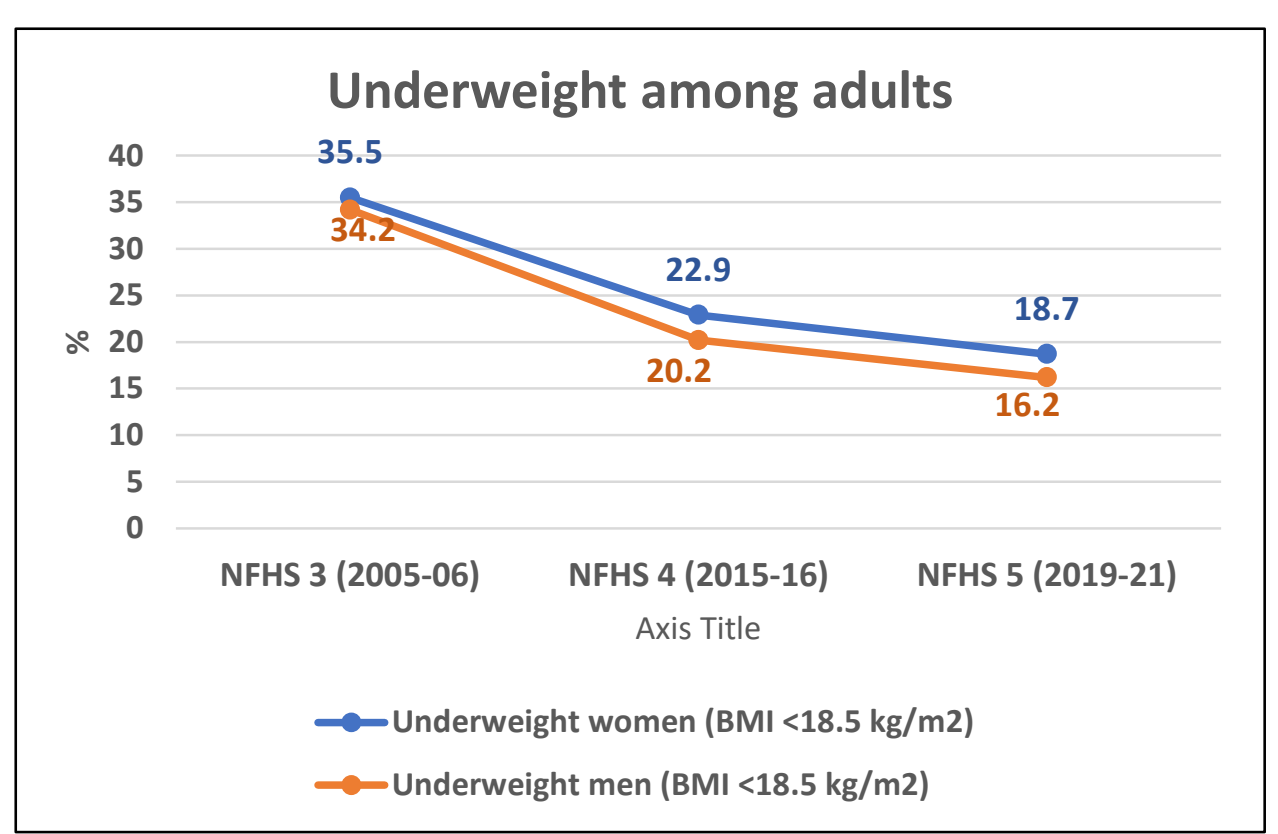

National trends

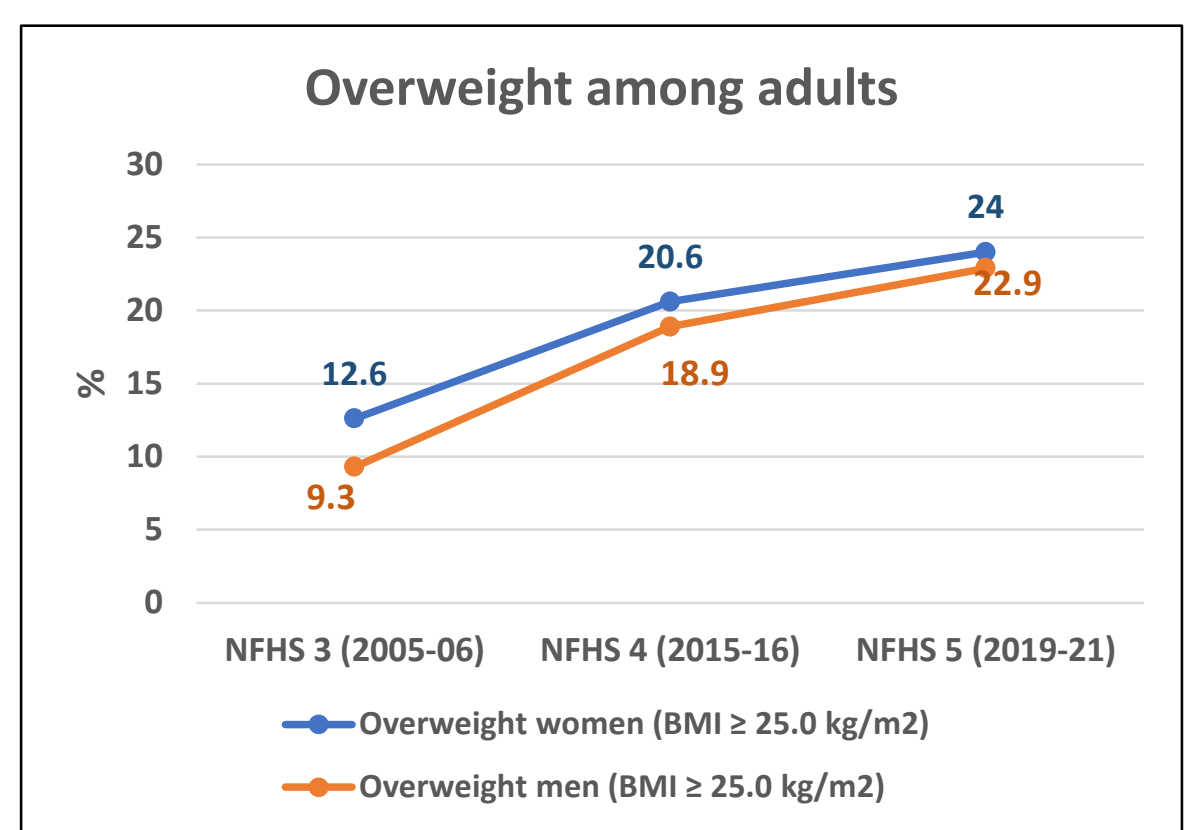

Click here for State findings

\begin{tabular}{|l|l|l|l|}
\hline Change over NFHS 4 & $\begin{array}{l}\text { Number of } \\
\text { States } \\
\text { improving }\end{array}$ & $\begin{array}{l}\text { Number of } \\
\text { States with } \\
\text { no change }\end{array}$ & $\begin{array}{l}\text { Number of } \\
\text { States } \\
\text { worsening }\end{array}$ \\
\hline Underweight women & 33 & 0 & 3 \\
\hline$\underline{\text { Underweight men }}$ & 27 & 0 & 9 \\
\hline Overweight women & 5 & 2 & 29 \\
\hline Overweight men & 2 & 1 & 33 \\
\hline
\end{tabular}




\section{Nutritional Status of adult women}

Change in prevalence of women who are underweight $(\mathrm{BMI}<18.5 \mathrm{~kg} / \mathrm{m} 2)$ and overweight or obese $(\mathrm{BMI} \geq 25.0 \mathrm{~kg} / \mathrm{m} 2)$ in India as per NFHS- 4 \&5 (2015-16 \& 2019-21)

\section{Underweight women (-4.2\%)}

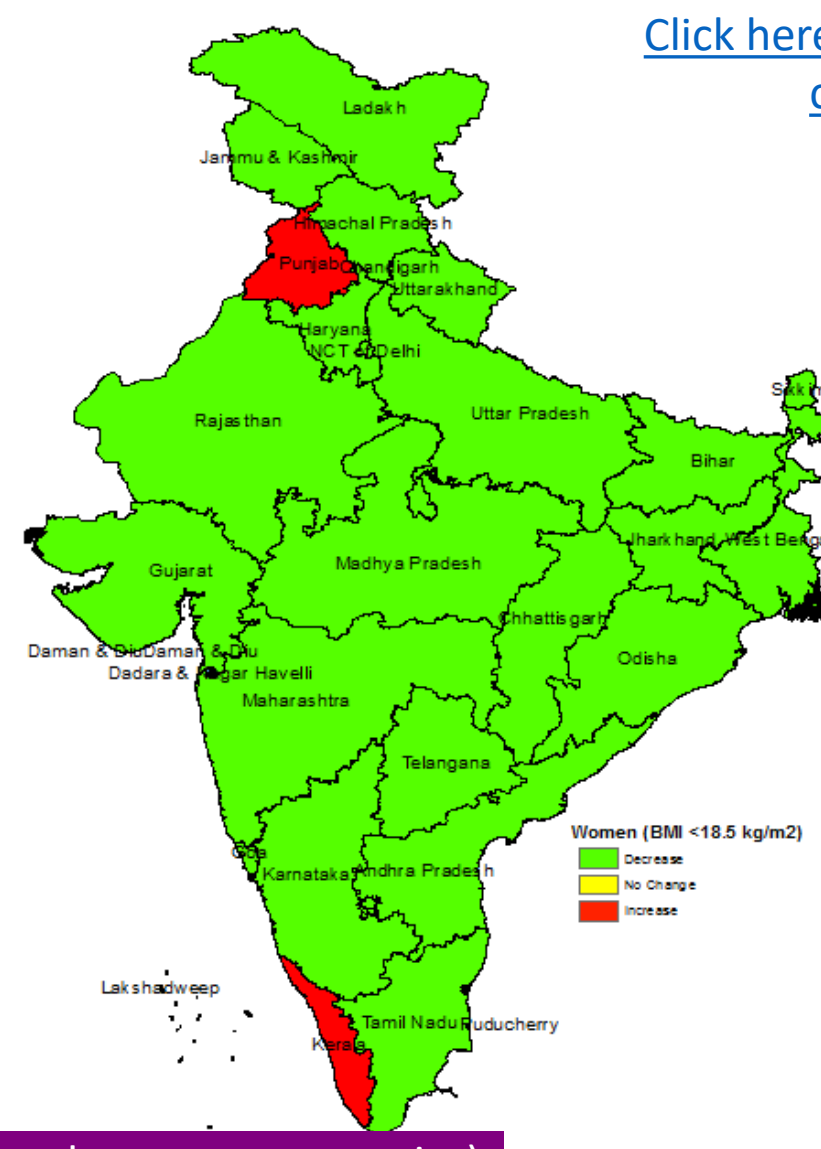

Top 5 states (based on change percent point)

Assam (-8.1 \%), Rajasthan (-7.3\%), Jammu \& Kashmir (-7\%), West Bengal (-6.5), Uttar Pradesh (-6.3\%)
Overweight women (+3.4\%)

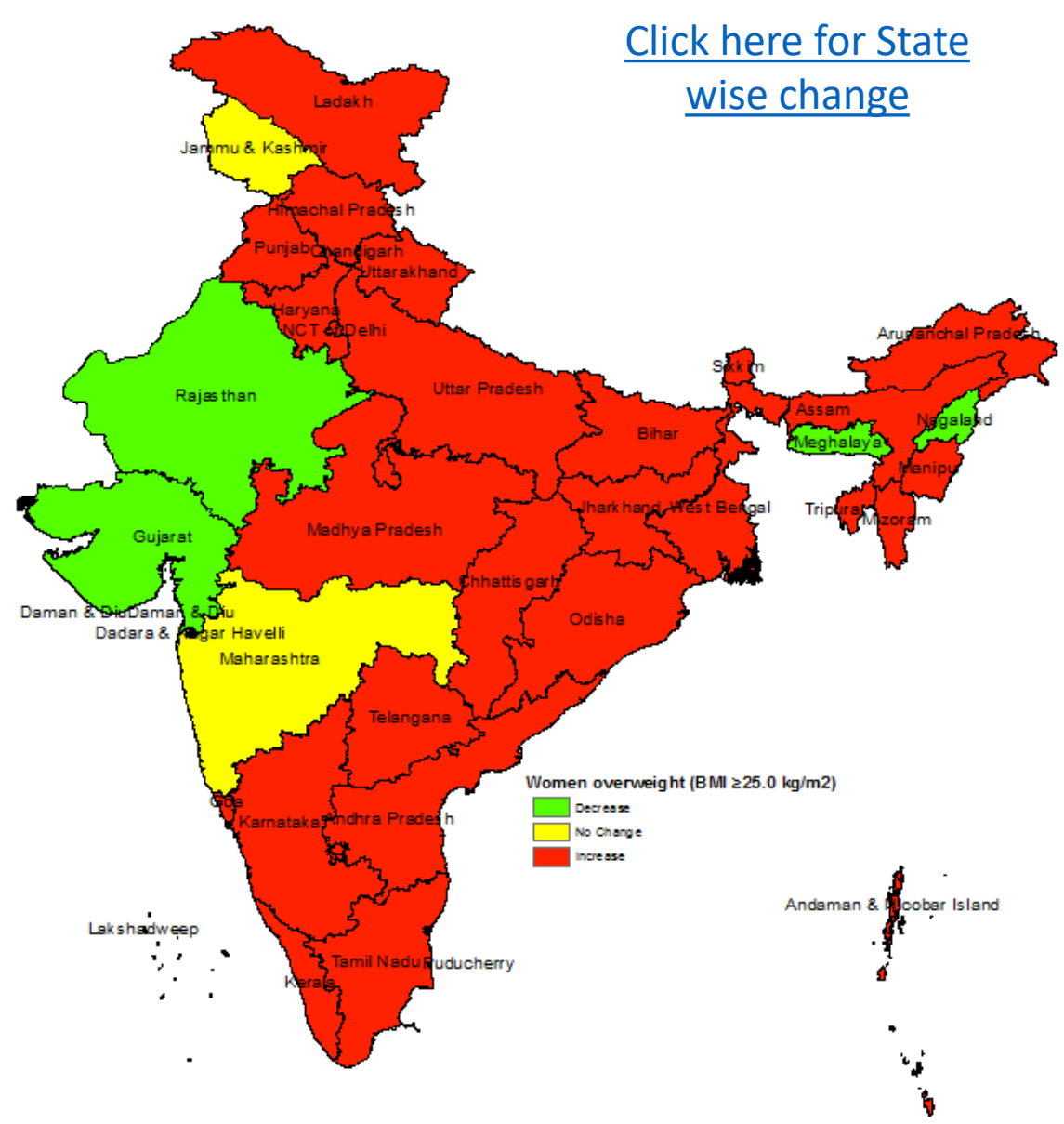

Lakshadweep (-7.1\%), Nagaland (-1.8\%), Rajasthan (-1.2\%), Gujarat (-1.1\%) Meghalaya (-0.7\%) 


\section{Nutritional Status of adult men}

Change in prevalence of men who are underweight (BMI $<18.5 \mathrm{~kg} / \mathrm{m} 2)$ and overweight or obese $(\mathrm{BMI} \geq 25.0 \mathrm{~kg} / \mathrm{m} 2)$ in India as per NFHS -4 \&5 (2015-16 \& 2019-21)

Underweight Men (-4\%)

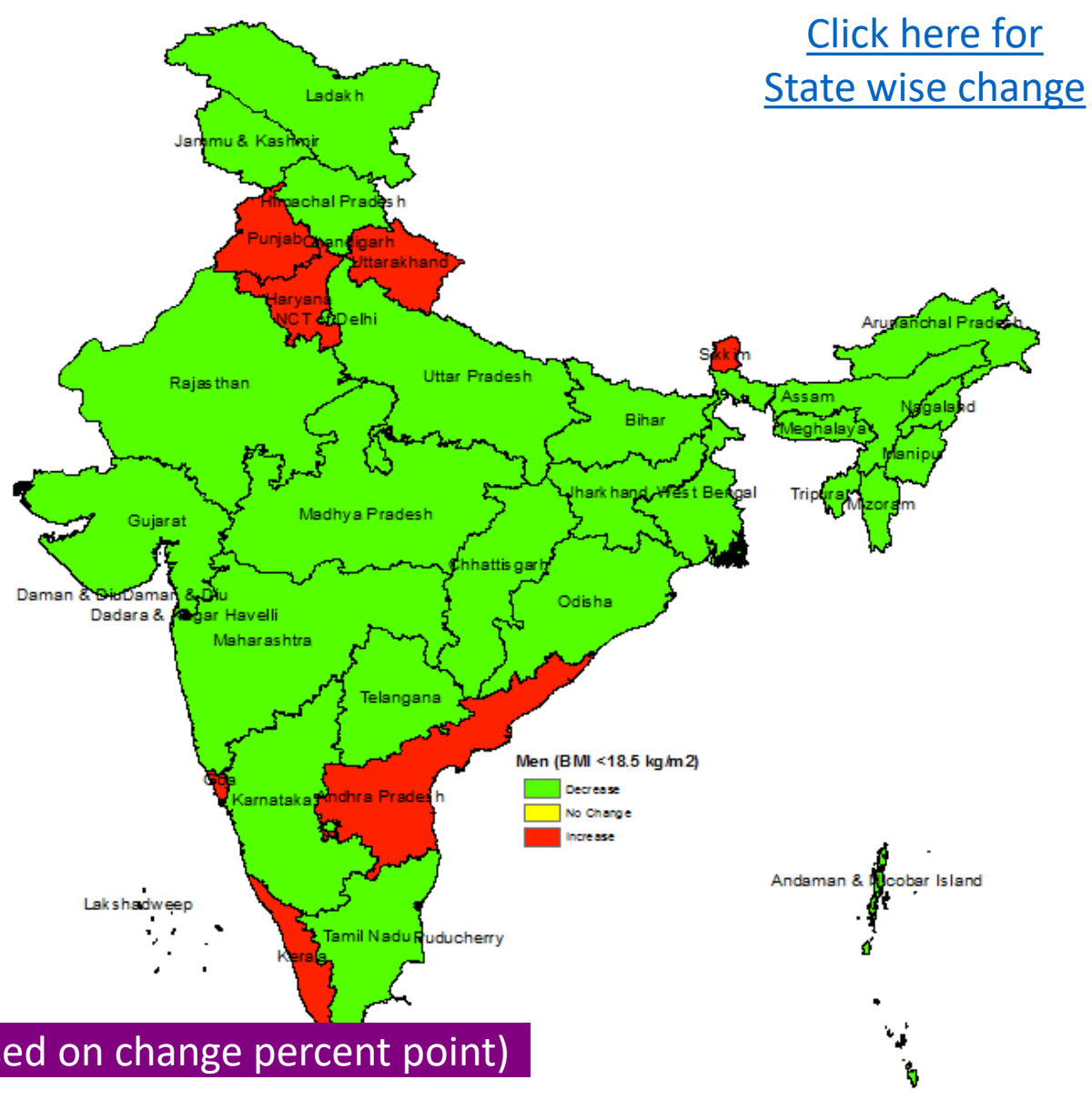

Ladakh (-9.1\%), Rajasthan (-8.7\%), NCT Delhi (-8.6\%),

Uttar Pradesh (-8\%), Madhya Pradesh (-7.6\%)

\section{Overweight Men (+4\%)}

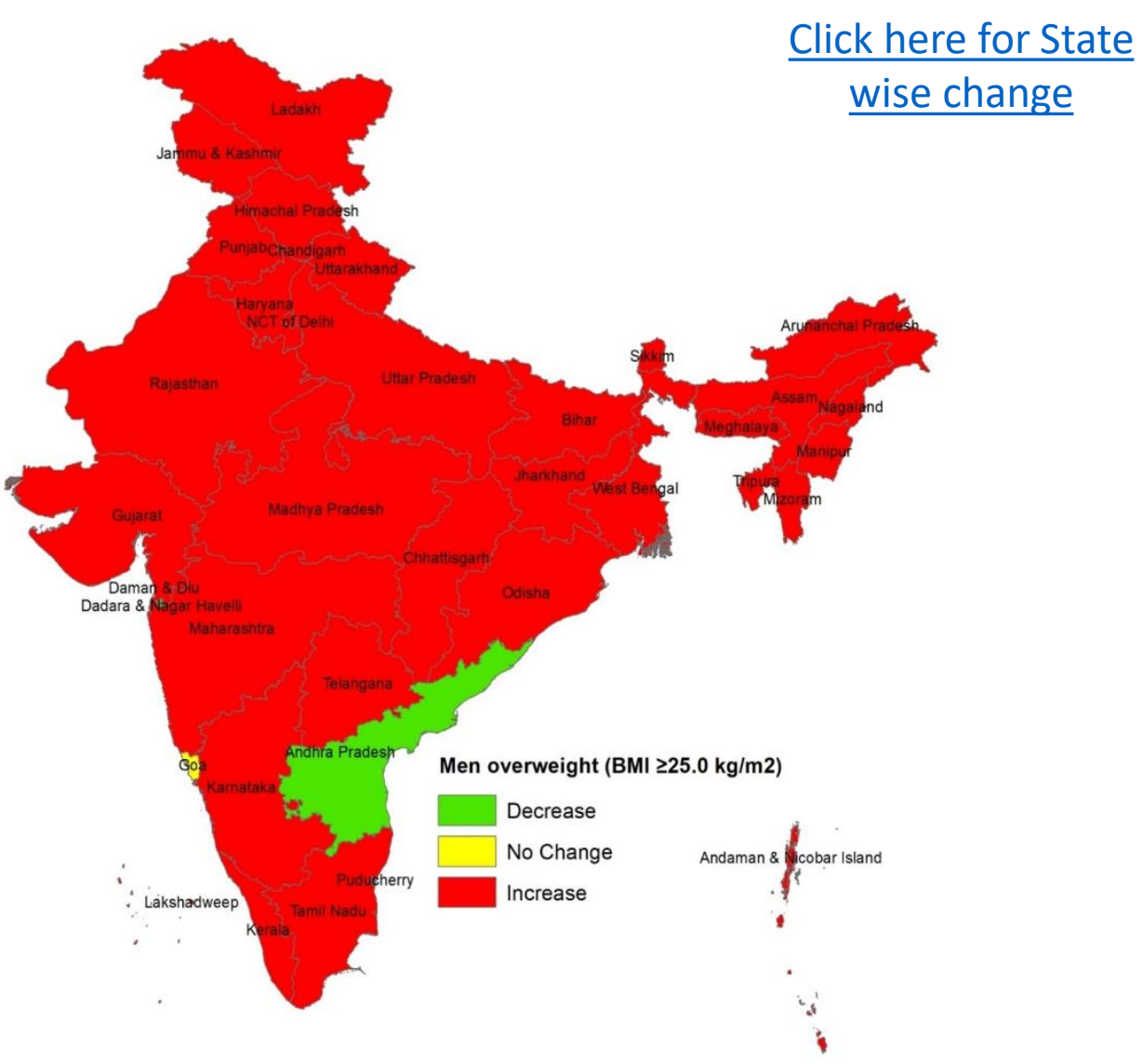

Dadra and Nagar Haveli \& Daman and Diu (-4.9\%), Andhra Pradesh (-2.4\%) 
Hypertension and Diabetes among adults

The prevalence of hypertension and diabetes is high nationally

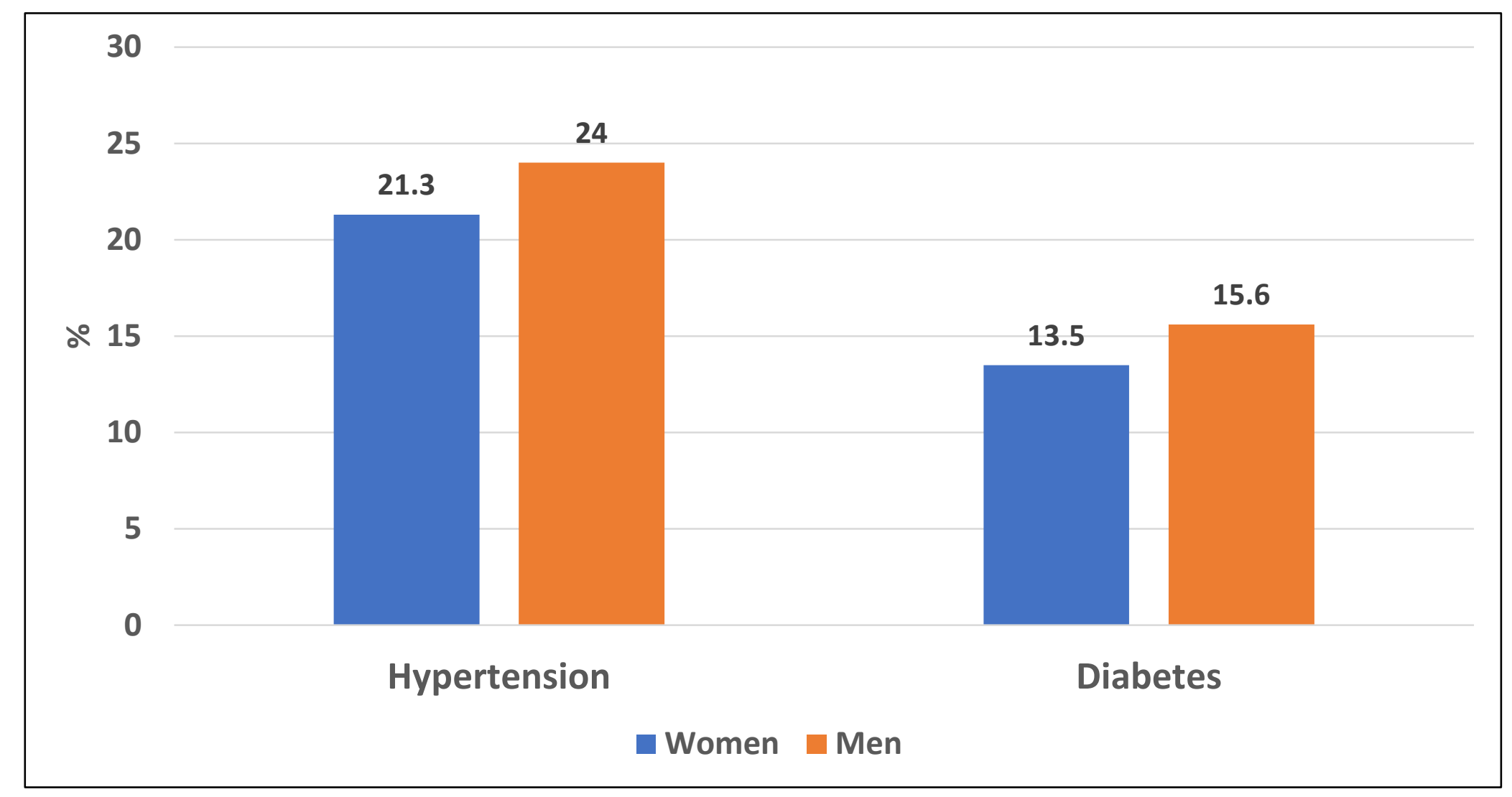

Click here for State findings 


\section{Anemia among children and adolescents}

\section{Worrying trends of anemia among children (6-59 months) and adolescents nationally.}

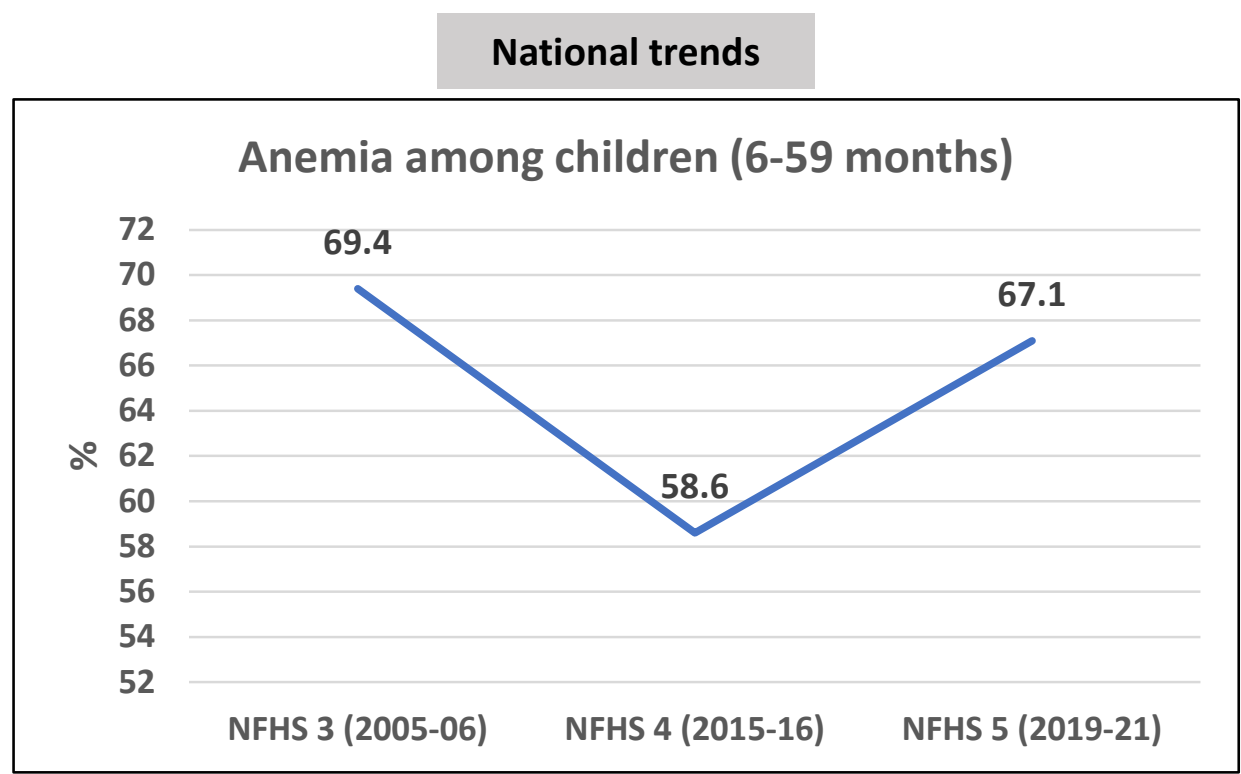

Anemia in children age 6-59 months defined as hemoglobin $<11.0 \mathrm{~g} / \mathrm{dl}$ )

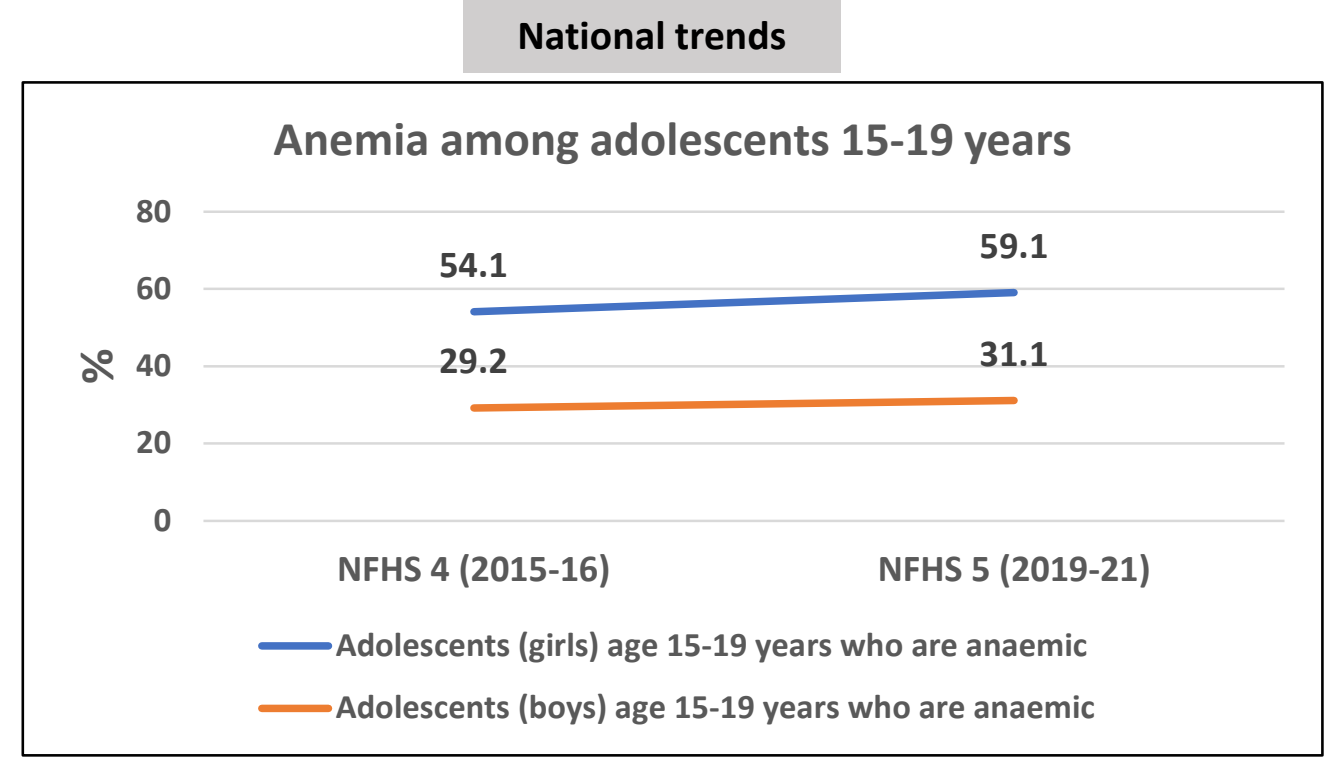

Anemia in adolescents age 15-19 years defined as hemoglobin $<12.0 \mathrm{~g} / \mathrm{dl}$ )

Click here for State findings

\begin{tabular}{|l|l|l|l|}
\hline Change over NFHS 4 & $\begin{array}{l}\text { Number of } \\
\text { States } \\
\text { improving }\end{array}$ & $\begin{array}{l}\text { Number of } \\
\text { States with } \\
\text { no change }\end{array}$ & $\begin{array}{l}\text { Number of } \\
\text { States } \\
\text { worsening }\end{array}$ \\
\hline Anemia in children (6-59 months) & 8 & 0 & 28 \\
\hline Anemia in adolescents (girls) 15-19 years & 12 & 0 & 24 \\
\hline Anemia in adolescents (boys) 15-19 years & 11 & 0 & 23 \\
\hline
\end{tabular}




\section{Anemia in children $\&$ adolescents}

Change in prevalence of anemia in children and adolescents in India as per NFHS - 4 \& 5 (2015-16 \& 2019-21)

\section{Anemia in children (+8.5\%)}

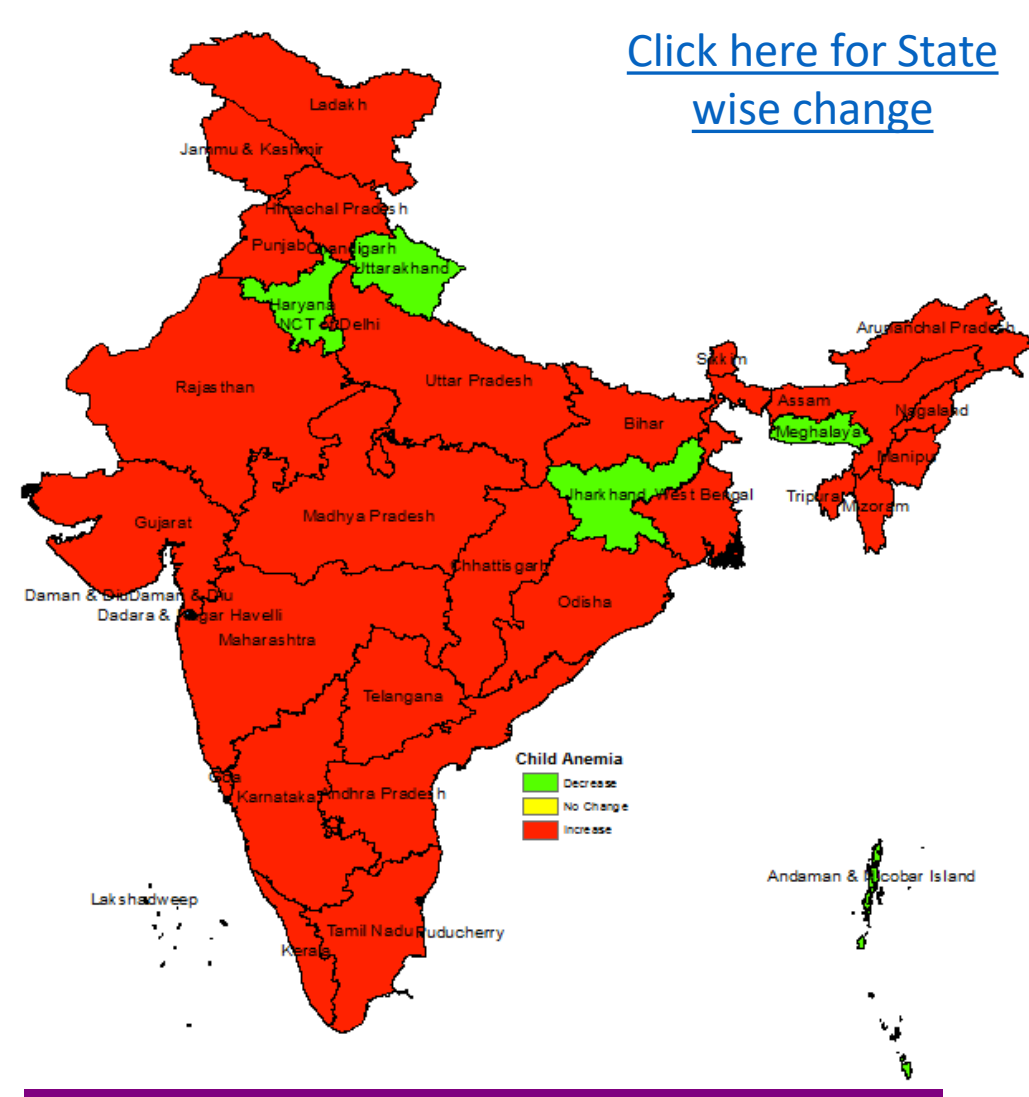

Top 5 states (based on change percent point)

Chandigarh (-18.5\%), Lakshadweep (-10.5\%), Andaman and Nicobar Island (-9\%), Dadra and Nagar Haveli \& Daman and Diu (-6.2\%), Meghalaya $(-2.9 \%)$

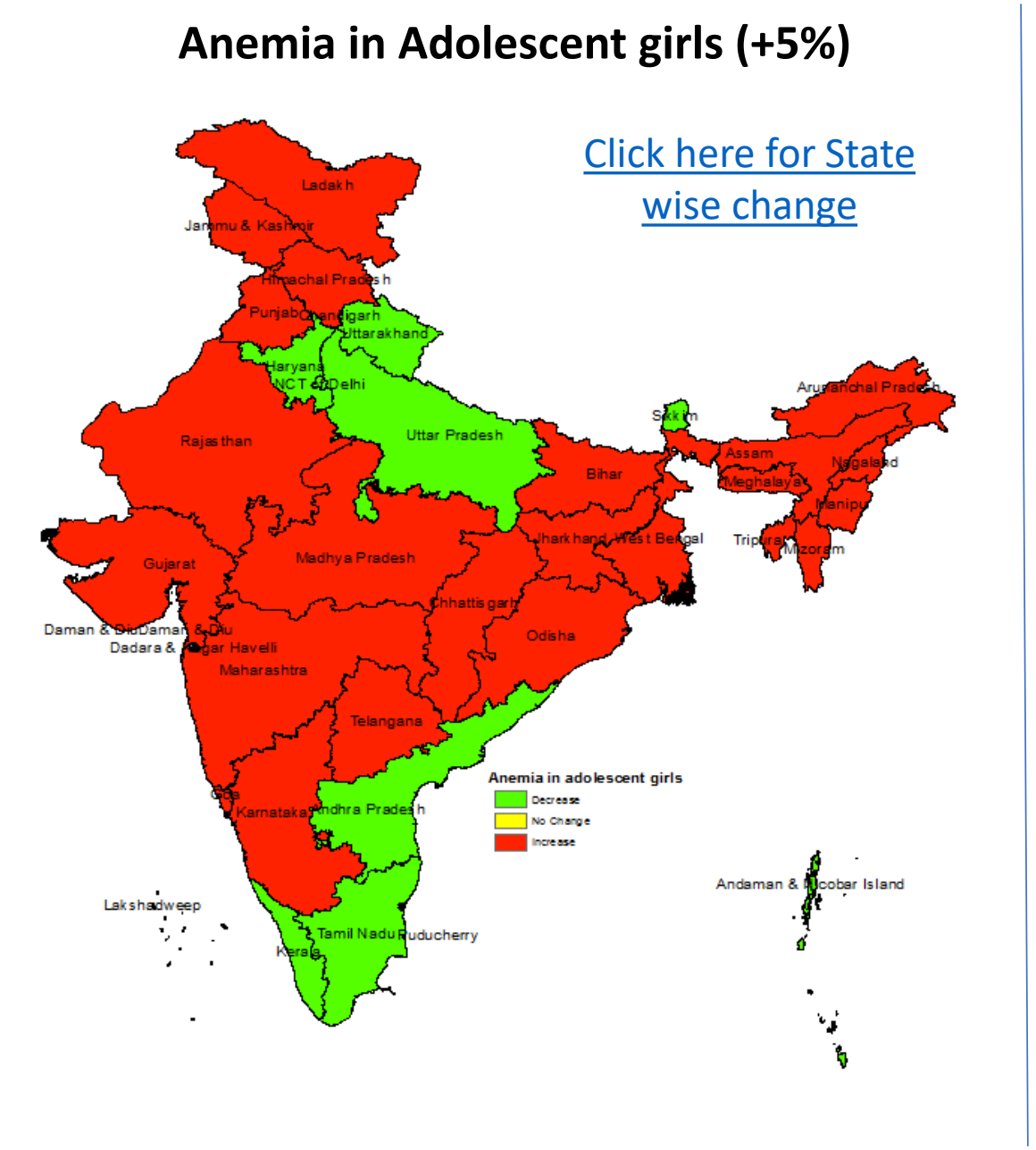

Lakshadweep (-27.6\%), Andaman \& Nicobar Island (-23.2\%), Chandigarh (-17\%), Dadra and Nagar Haveli \& Daman and Diu (-12\%), Uttarakhand (-5.5\%)
Anemia in Adolescent boys (+1.9\%)

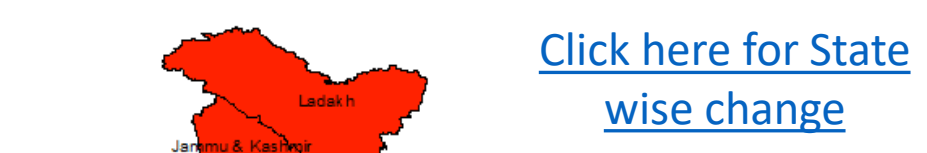

\section{wise change}

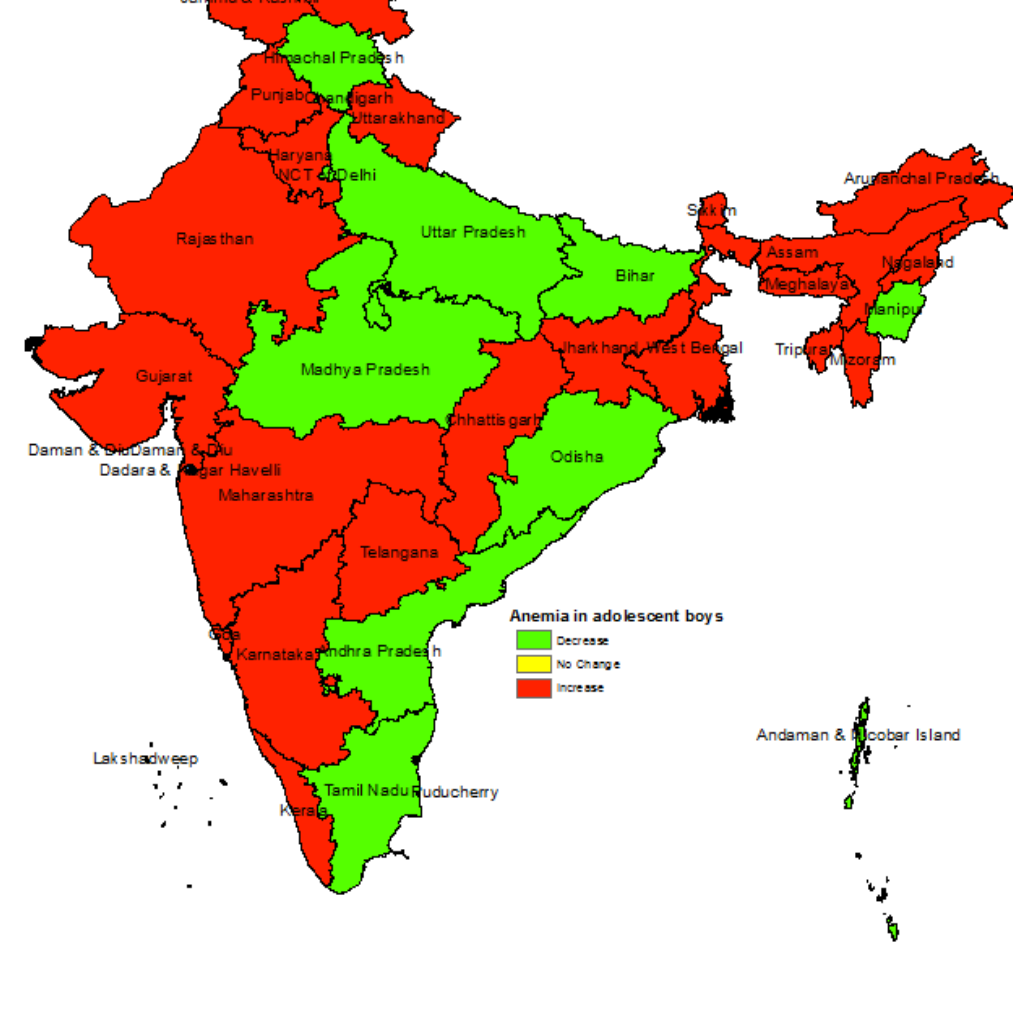

Andaman \& Nicobar Island (-15.9\%), Andhra Pradesh (-10.6\%), Puducherry (-9.9\%), NCT Delhi (-7\%), Madhya Pradesh (-6\%) 


\section{Anemia among men and women}

\section{An increase was observed in anemia among adult women and men nationally.}

\section{National trends}

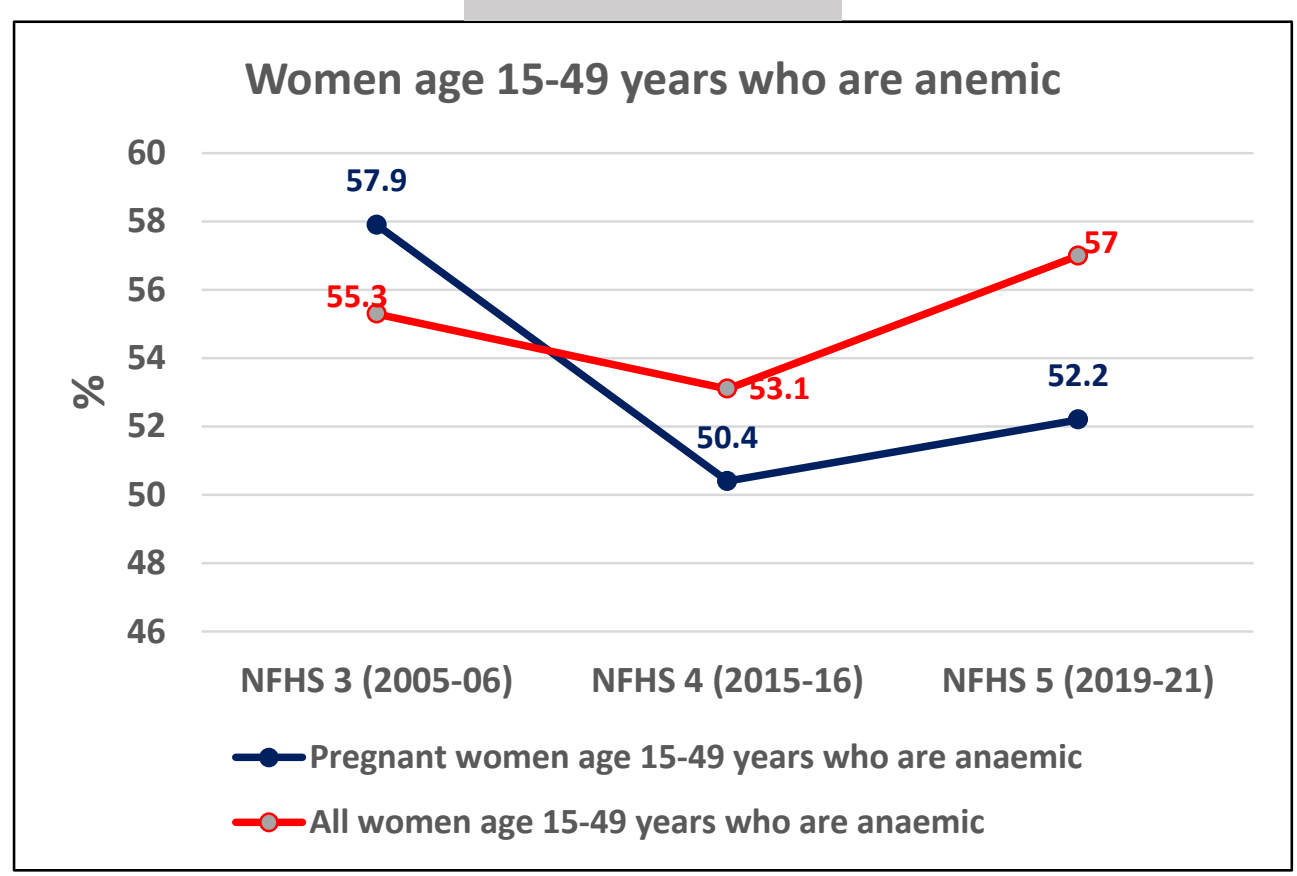

Anemia in pregnant women defined as hemoglobin $<11.0 \mathrm{~g} / \mathrm{dl}$ Anemia in adult women defined as hemoglobin $<12.0 \mathrm{~g} / \mathrm{dl}$

\section{National trends}

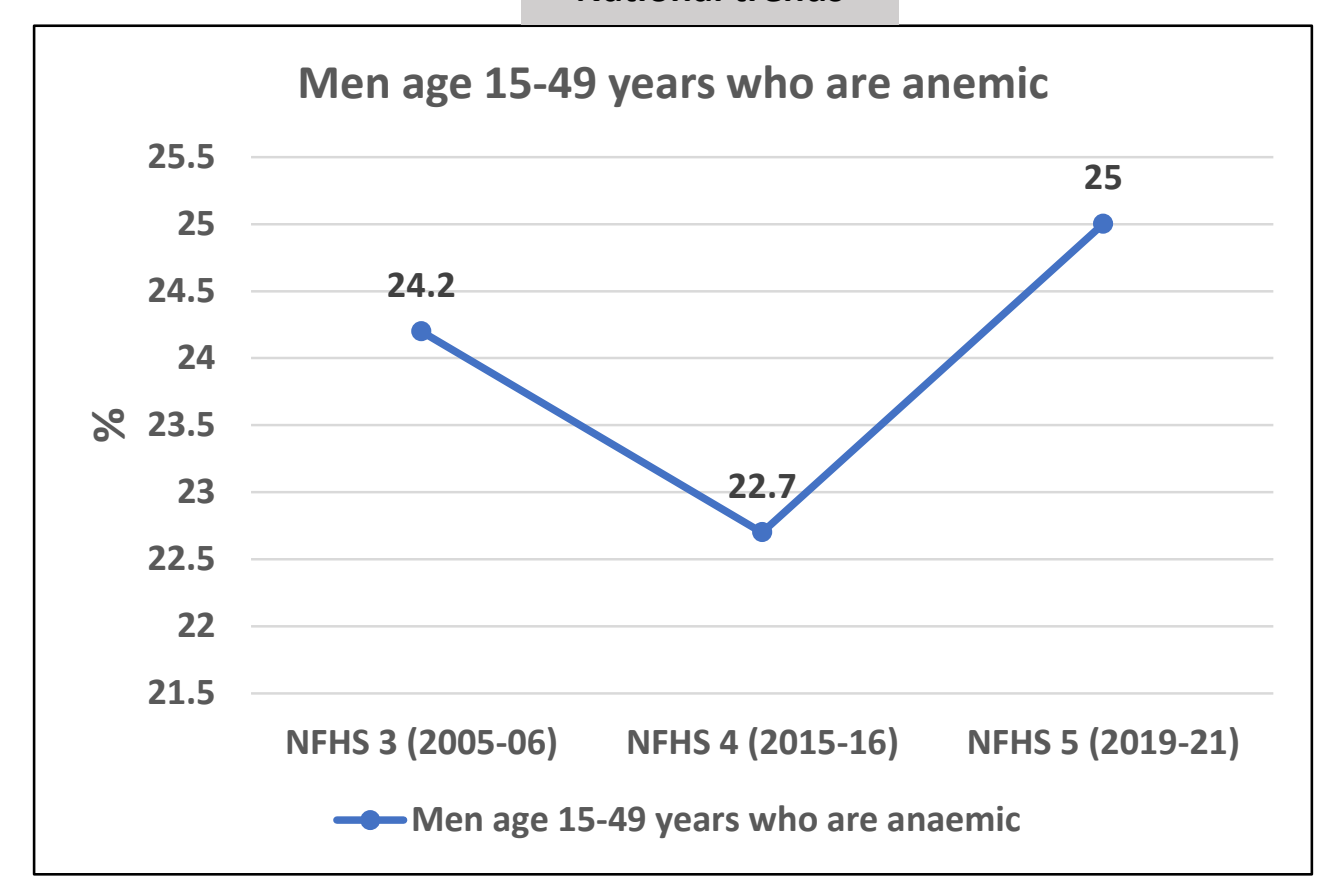

Anemia in adult men age $15-49$ years defined as hemoglobin $<13.0 \mathrm{~g} / \mathrm{dl}$

Click here for State findings

\begin{tabular}{|l|l|l|l|}
\hline Change over NFHS 4 & $\begin{array}{l}\text { Number of } \\
\text { States } \\
\text { improving }\end{array}$ & $\begin{array}{l}\text { Number of } \\
\text { States with } \\
\text { no change }\end{array}$ & $\begin{array}{l}\text { Number of } \\
\text { States } \\
\text { worsening }\end{array}$ \\
\hline Anemia in pregnant women age 15-49 years & 16 & 0 & 19 \\
\hline Anemia in all women age 15-49 years & 13 & 0 & 23 \\
\hline Anemia in men age 15-49 vears & 18 & 1 & 17 \\
\hline
\end{tabular}




\section{Anemia in adults}

Change in prevalence of anemia in all women aged 15-49 years, pregnant women and men aged 15-

49 years in India as per NFHS - 4 \& 5 (2015-16 \& 2019-21)

\section{Anemia in all women $(+3.9 \%)$}

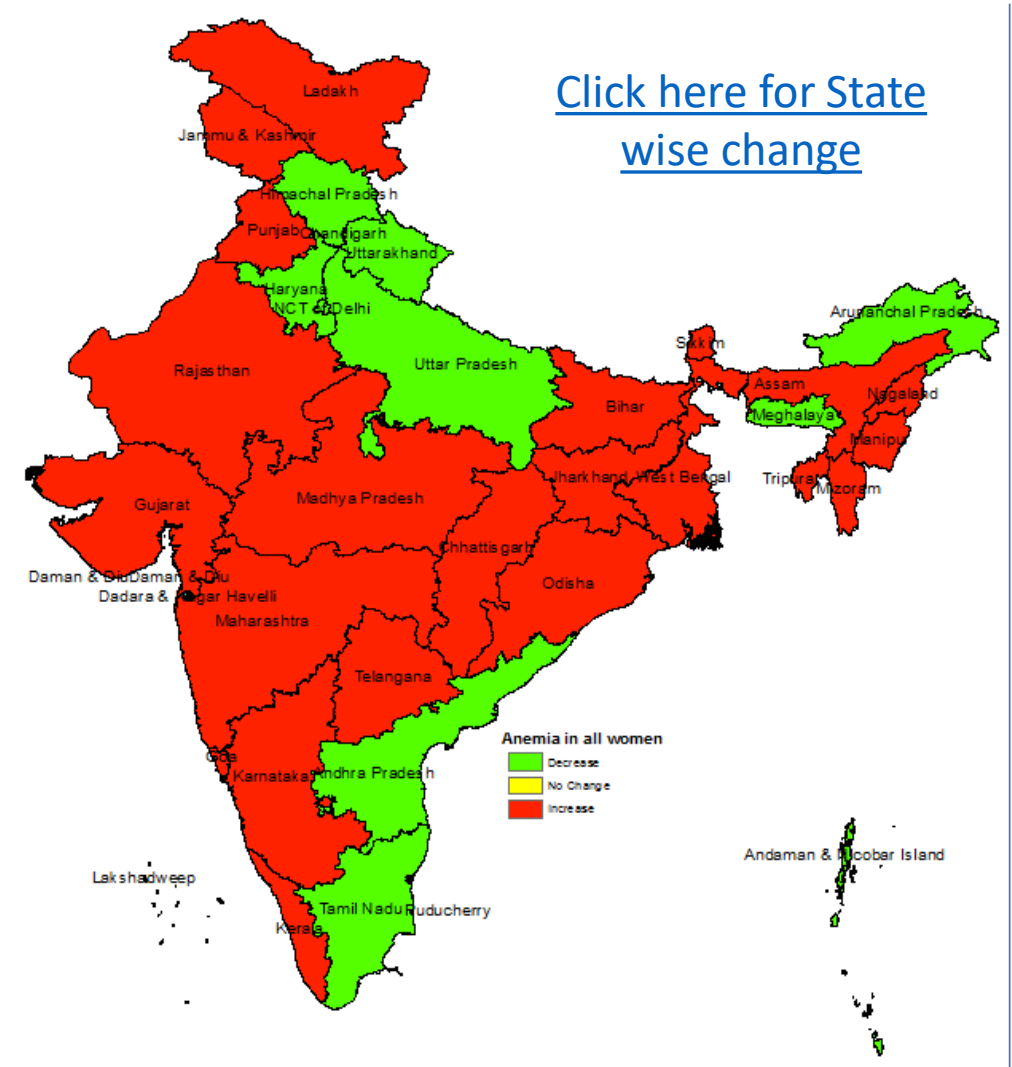

Top 5 states (based on change percent point)

Lakshadweep (-20.2\%), Chandigarh (-15.6\%), Dadra and Nagar Haveli \& Daman and Diu

$(-10.4 \%)$, Andaman \& Nicobar Island (-8.2), NCT Delhi (-4.4\%)

Back to contents
Anemia in Pregnant women (+1.8\%)

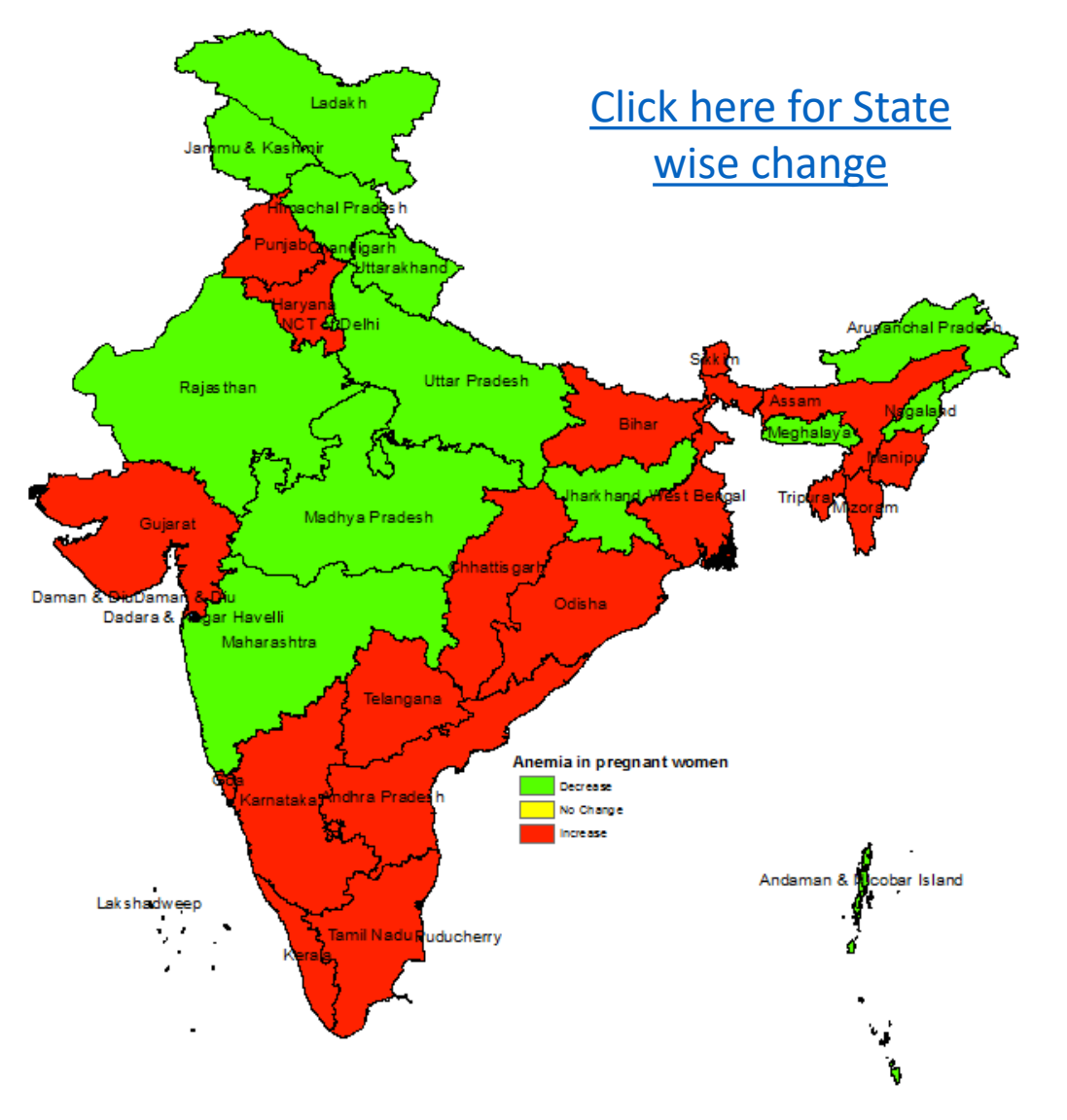

Lakshadweep (-18.1\%), Nagaland (-10.5\%),

Arunachal Pradesh (-9.9\%), Meghalaya (-8.3\%),

Himachal Pradesh (-8.2\%)
Anemia in men (+2.3\%)

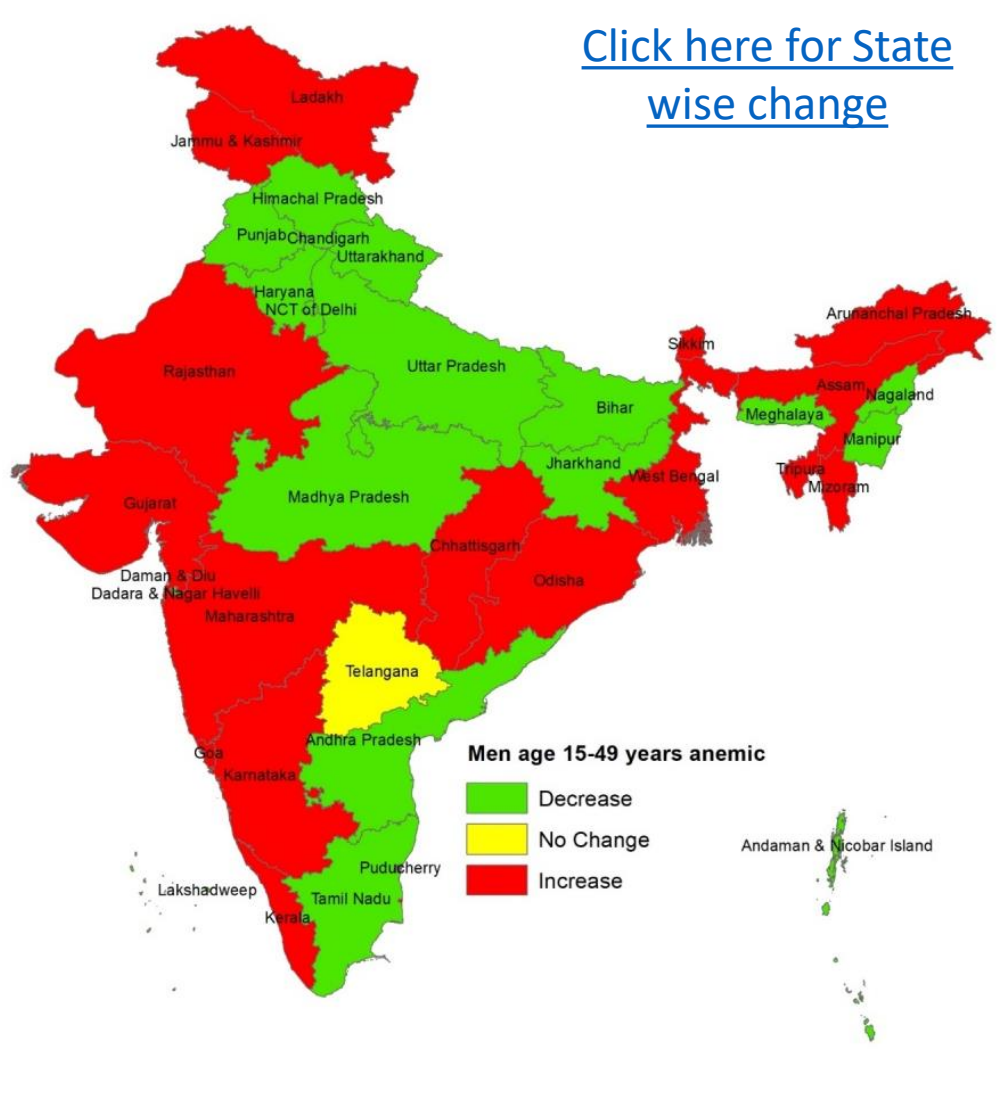

Andaman and Nicobar (-14.7\%), Chandigarh (-11.2\%), Andhra Pradesh (-10.8\%), NCT Delhi (-9.1), Meghalaya (-6.9) 


\section{Consumption of vitamin \& mineral supplements}

Iron Folic acid(IFA) supplements consumption among pregnant women and vitamin A supplementation among children has increased nationally.
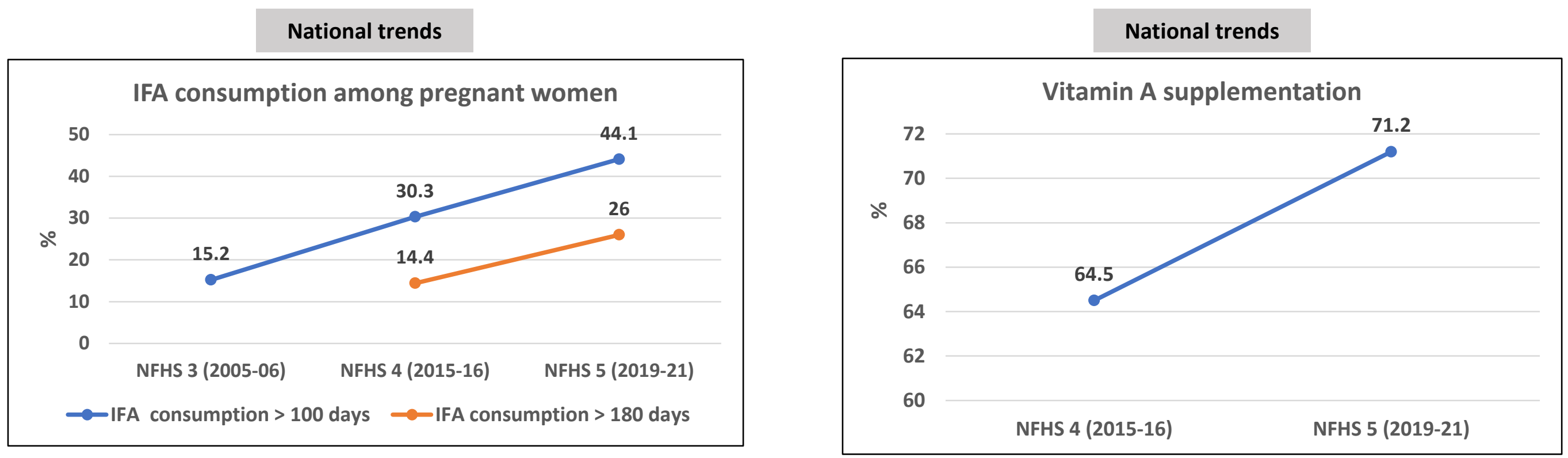

\begin{tabular}{|c|c|c|c|}
\hline \multicolumn{4}{|c|}{ Click here for State findings } \\
\hline Change over NFHS 4 & $\begin{array}{l}\text { Number of States } \\
\text { improving }\end{array}$ & $\begin{array}{l}\text { Number of } \\
\text { States with no } \\
\text { change }\end{array}$ & $\begin{array}{l}\text { Number of } \\
\text { States } \\
\text { worsening }\end{array}$ \\
\hline $\begin{array}{l}\text { Mothers who consumed IFA for } 100 \text { days } \\
\text { or more when they were pregnant }\end{array}$ & 32 & 0 & 4 \\
\hline $\begin{array}{l}\text { Mothers who consumed iron folic acid for } \\
180 \text { days or more when they were } \\
\text { pregnant }\end{array}$ & 33 & 0 & 3 \\
\hline $\begin{array}{l}\text { Vitamin A supplementation among } \\
\text { children }\end{array}$ & 26 & 0 & 10 \\
\hline
\end{tabular}




\section{Consumption of vitamin \& mineral supplements}

Change in prevalence of consumption of IFA for 100 days and 180 days among pregnant women and vitamin A dose among children in India as per NFHS - 4 \&5 (2015-16 \& 2019-21)

\section{IFA for 100 days (+13.8\%)}

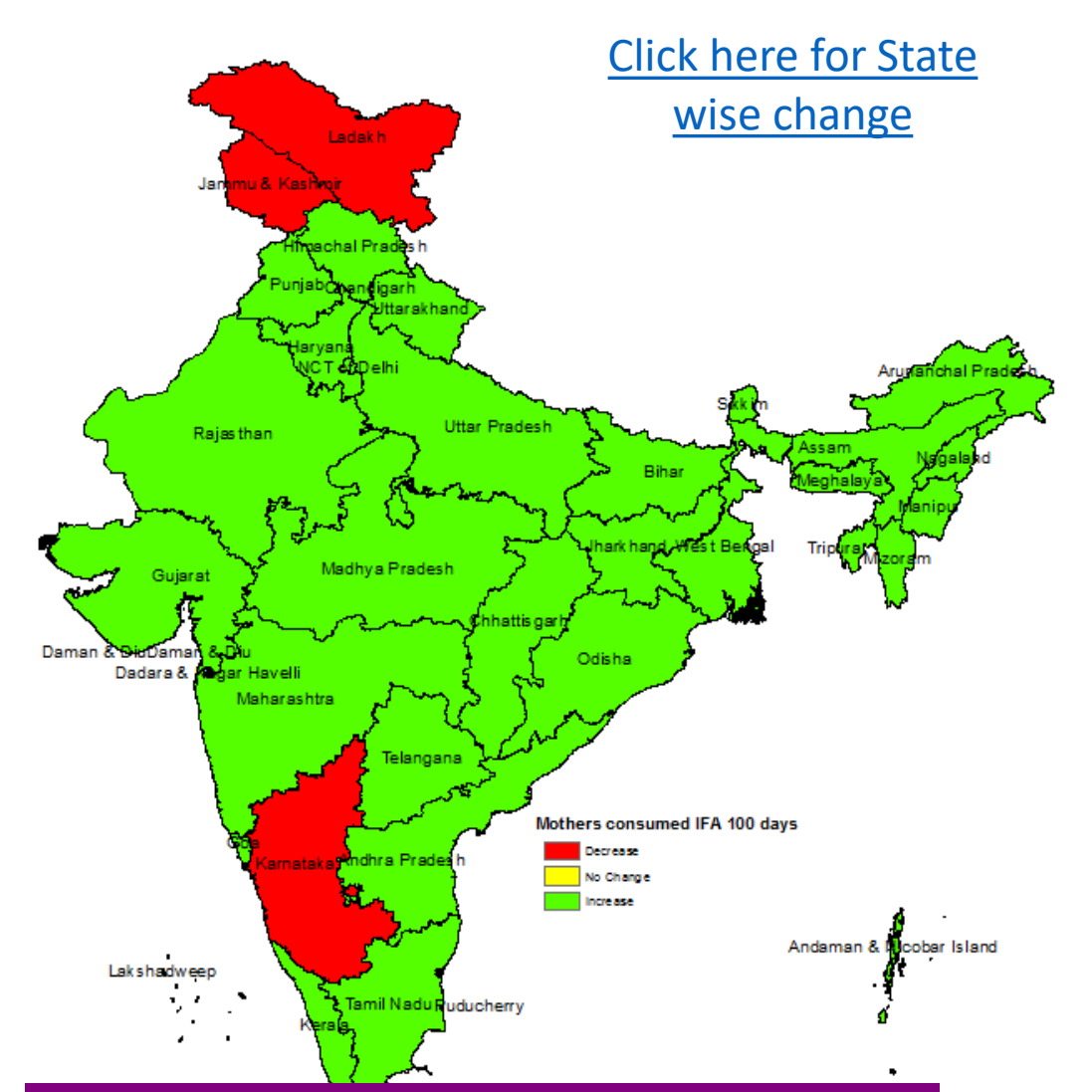

\section{Top 5 states (based on change percent point)}

West Bengal (+34.5\%), Chandigarh (29\%),

Madhya Pradesh (+27.9\%), Odisha $(+24.3 \%)$,

Gujarat (+23.2\%)

Back to contents

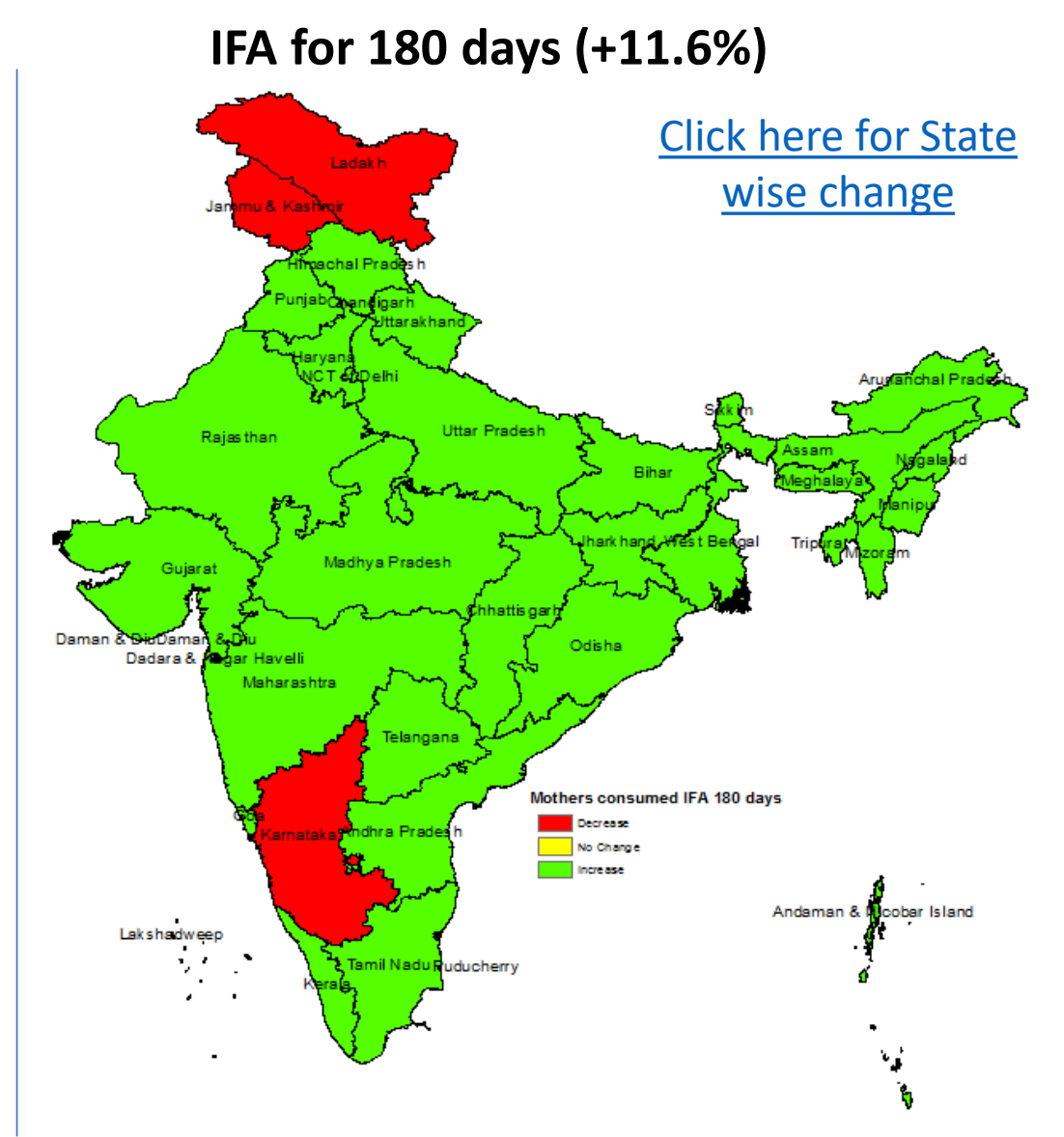

Chandigarh (+34.7\%), Odisha (+30.2\%)

Puducherry $(+28.6 \%)$, West Bengal $(+24.8 \%)$,

Gujarat (+24.7\%)

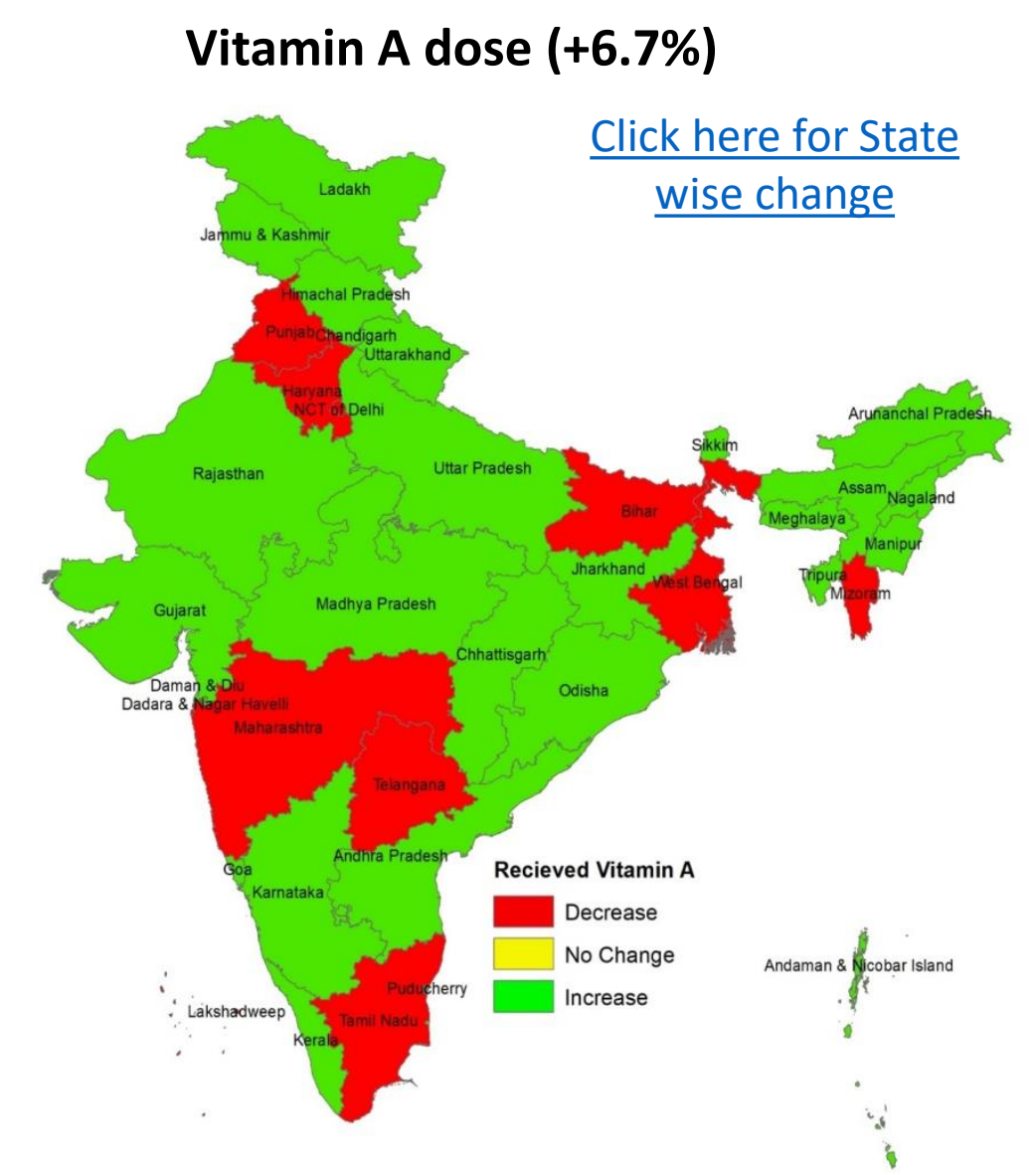

Uttar Pradesh (+ 30.1\%), Arunachal Pradesh (+24.4\%), Rajasthan (+20.5\%), Dadra \& Nagar Haveli \& Daman \& Diu (+ 19\%) 


\section{India observed an improvement in antenatal and postnatal care of mothers.}
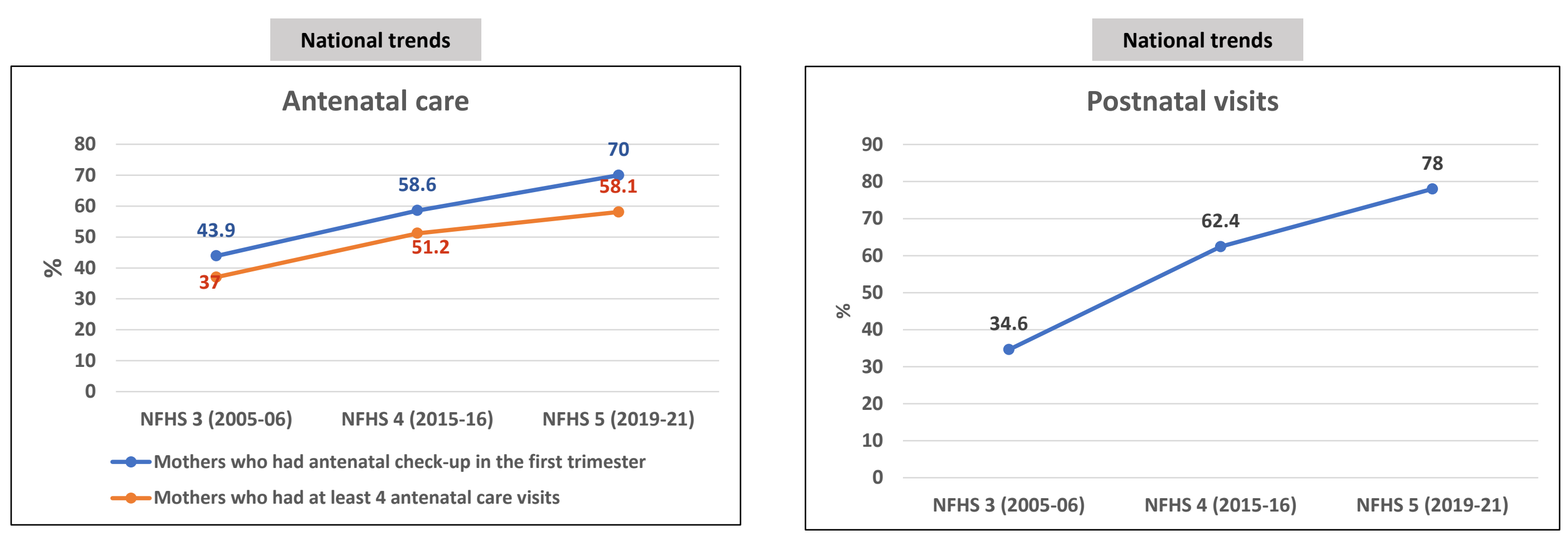

Click here for State findings

\begin{tabular}{|l|l|l|l|}
\hline Change over NFHS 4 & $\begin{array}{l}\text { Number of States } \\
\text { improving }\end{array}$ & $\begin{array}{l}\text { Number of States } \\
\text { with no change }\end{array}$ & $\begin{array}{l}\text { Number of } \\
\text { States } \\
\text { worsening }\end{array}$ \\
\hline $\begin{array}{l}\text { Mothers who had an antenatal check-up in } \\
\text { the first trimester }\end{array}$ & 29 & 0 & 7 \\
\hline $\begin{array}{l}\text { Mothers who had at least 4 antenatal care } \\
\text { visits }\end{array}$ & 23 & 0 & 13 \\
\hline $\begin{array}{l}\text { Mothers who received postnatal care } \\
\text { within 2 days of delivery }\end{array}$ & 31 & 0 & 5 \\
\hline
\end{tabular}




\section{Antenatal and postnatal care of mothers}

Change in Prevalence of mothers who had an antenatal checkup in the first trimester, had at least 4 antenatal care visits \& mothers who received post natal care in India as per NFHS - 4\&5 (2015-16 \& 2019-21)

\section{Antenatal checkup in $1^{\text {st }}$ trimester $(+11.4 \%)$}

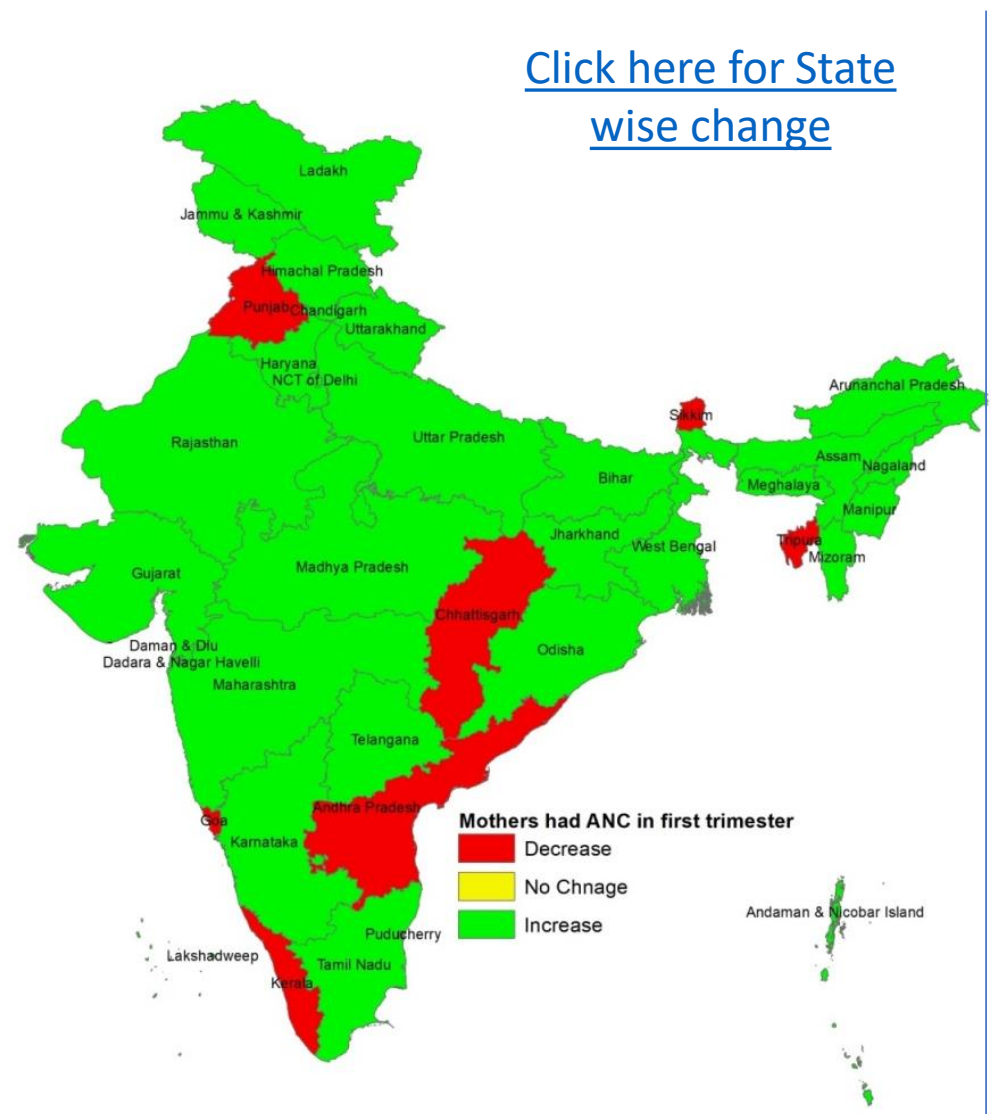

Top 5 states (based on change percent point)

Nagaland (+24.8\%), Madhya Pradesh (+22.4\%), Haryana $(+22 \%)$, Bihar $(+18.3 \%)$, West Bengal $(+17.7 \%)$

\section{At least 4 Antenatal care visits (+6.9\%)}

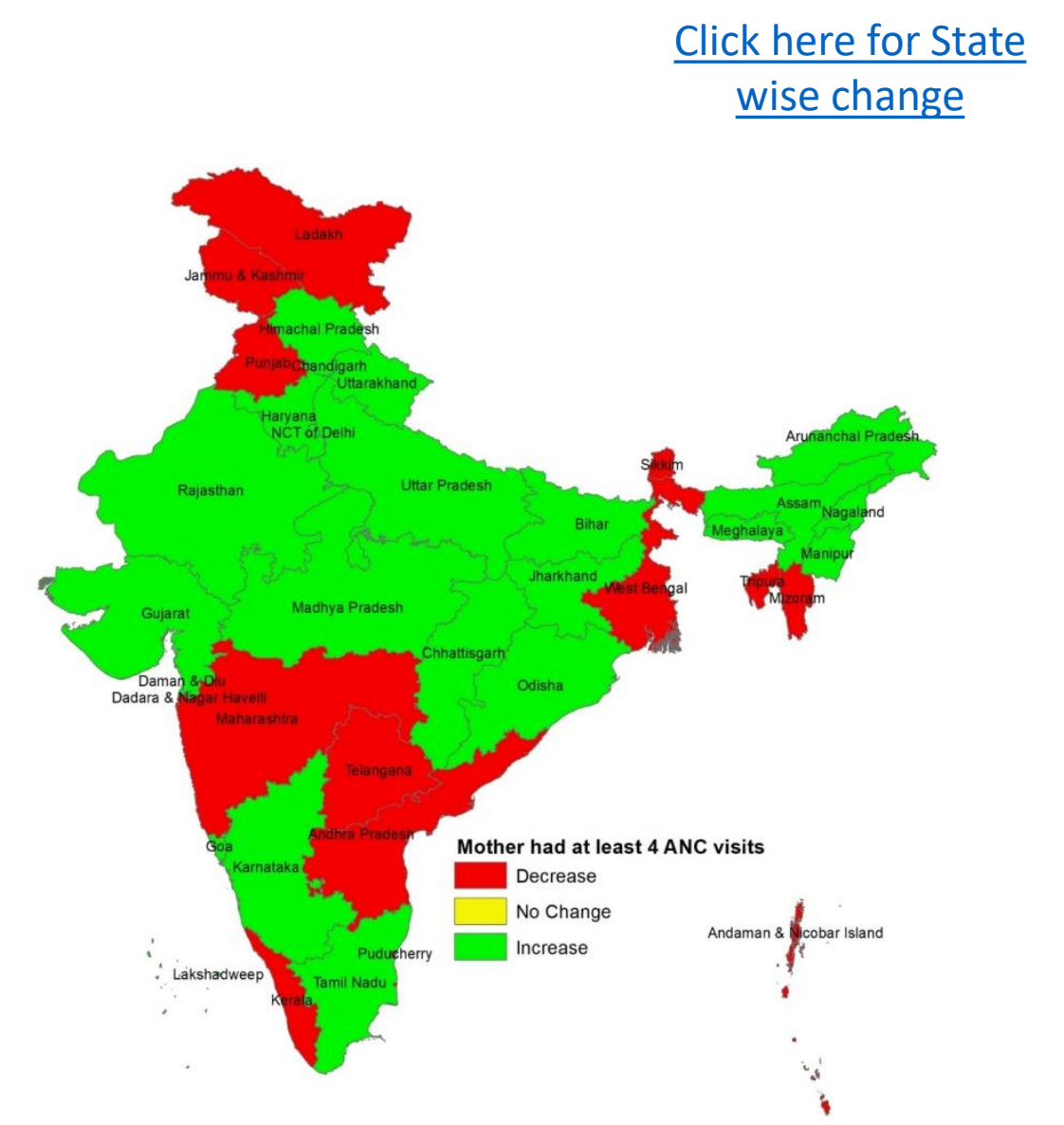

Uttarakhand (+ $30.9 \%)$, Madhya Pradesh (+ $21.8 \%)$, Rajasthan (+16.8\%), Odisha (+16.2\%), Uttar Pradesh $(+16.0 \%)$
Mothers receiving postnatal care within 2 days of delivery (+ $15.6 \%$ )

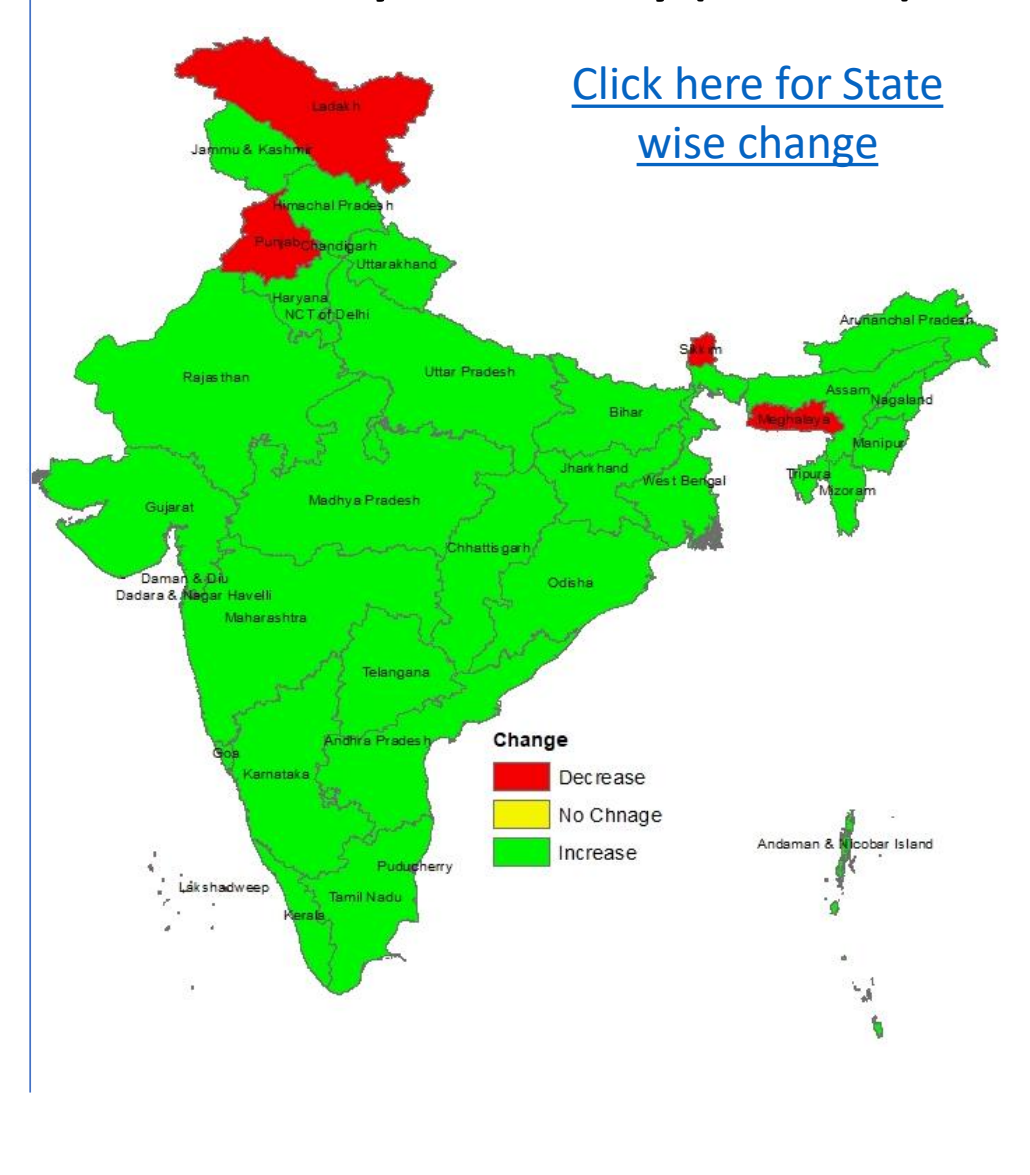

Madhya Pradesh (+28.6\%), Arunachal Pradesh (+27.6\%), Dadra and Nagar Haveli \& Daman and Diu (+26.8\%), Gujarat (+26.4\%), Jharkhand $(+24.7 \%)$ 


\section{Delivery care}

\section{India observed an increase in institutional and caesarian deliveries.}

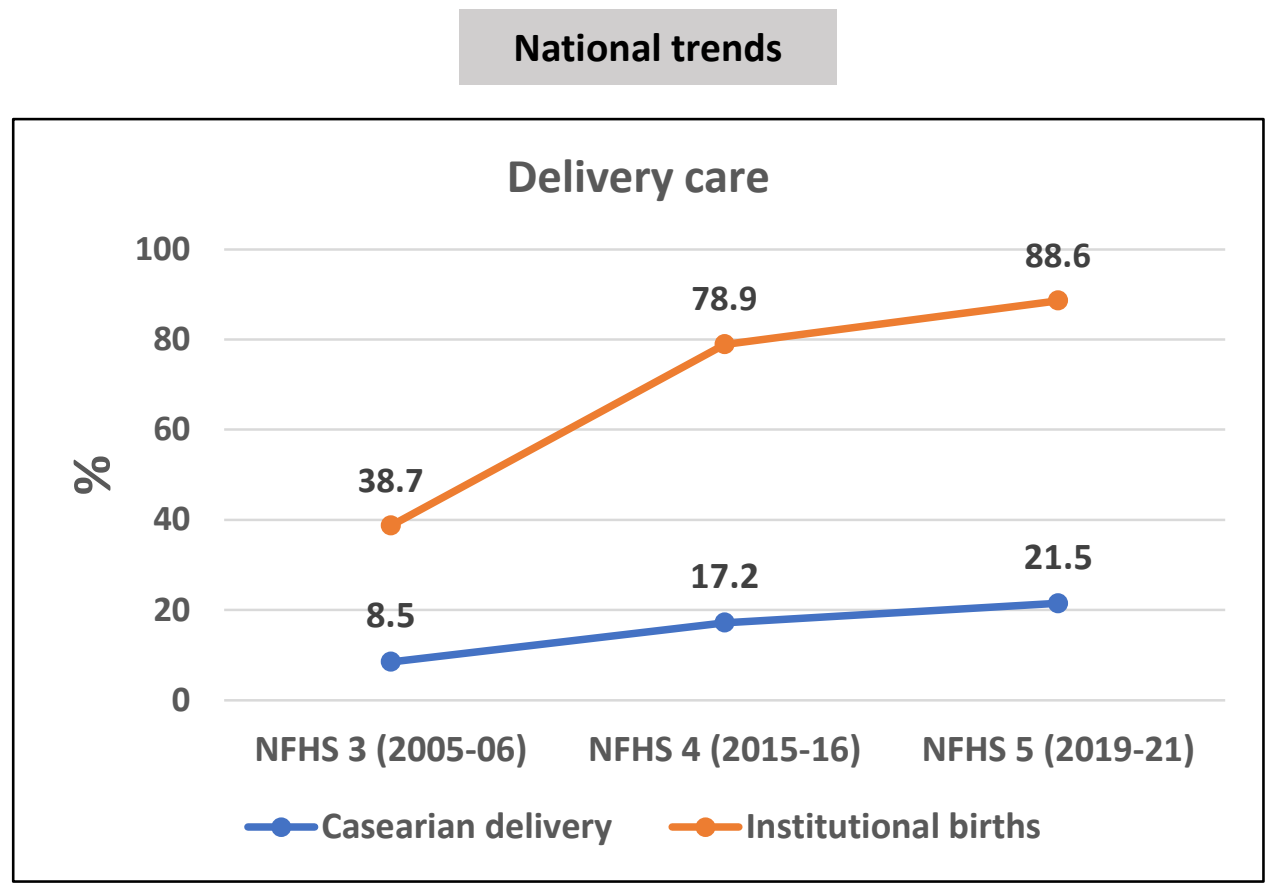

\begin{tabular}{|l|l|l|l|}
\hline Click here for State findings \\
\hline Change over NFHS 4 & $\begin{array}{l}\text { Number of } \\
\text { States } \\
\text { improving }\end{array}$ & $\begin{array}{l}\text { Number } \\
\text { of States } \\
\text { with no } \\
\text { change }\end{array}$ & $\begin{array}{l}\text { Number of } \\
\text { States } \\
\text { worsening }\end{array}$ \\
\hline Institutional births & 33 & 2 & 1 \\
\hline Caesarean delivery & 4 & 0 & 32 \\
\hline
\end{tabular}




\section{Delivery care}

Change in Prevalence of institutional births and caesarean delivery

India NFHS - 4 \&5 (2015-16 \& 2019-21)

Institutional births (+9.7\%)

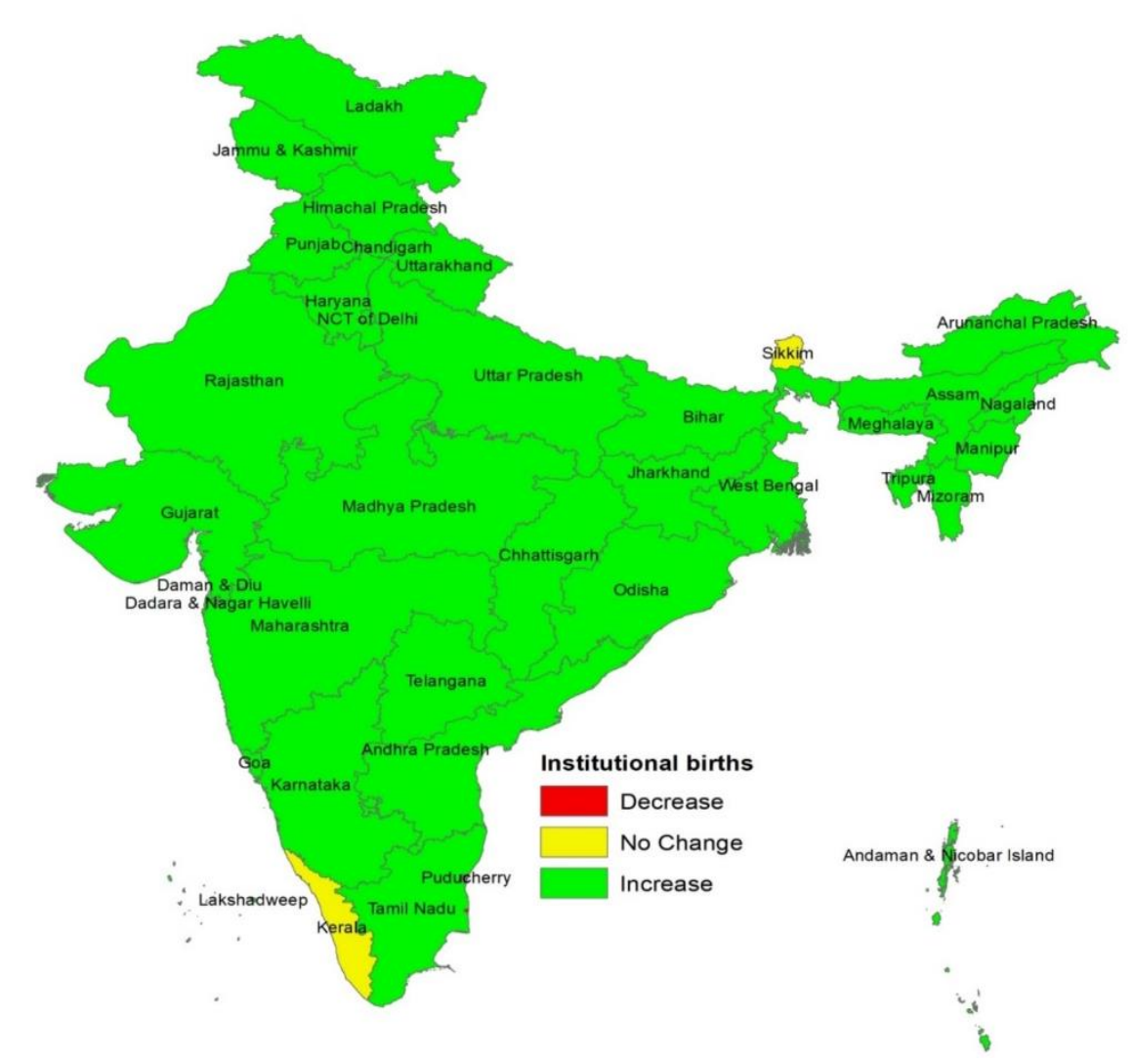

Top 5 states (based on change percent point)

Arunachal Pradesh (+27\%), West Bengal (+16.5\%), Uttar Pradesh $(+15.6 \%)$, Chhattisgarh (+15.5), Uttarakhand (+14.6\%) $\underline{\text { Click here for State }}$ wise change
Caesarean delivery (+4.3\%)

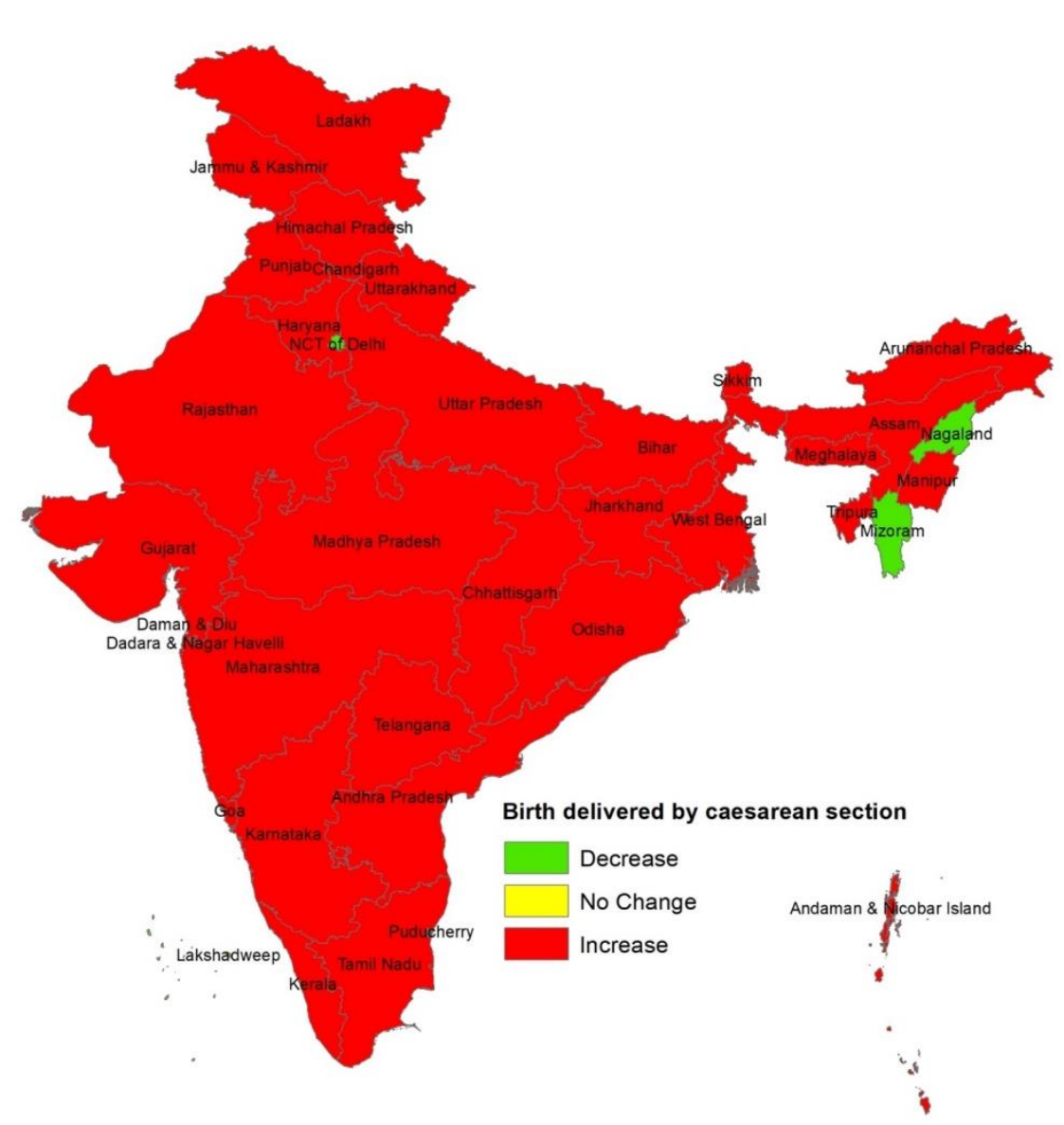

Lakshadweep (-7.1\%), NCT of Delhi (-3.1\%), Mizoram (-1.9\%), Nagaland (-0.6\%)
Click here for State wise change 
- A decrease was observed in the prevalence of diarrhoea among children under 5 years, nationally.

- A small increase in the prevalence of symptoms of acute respiratory infection (ARI) among children under 5 years was observed.

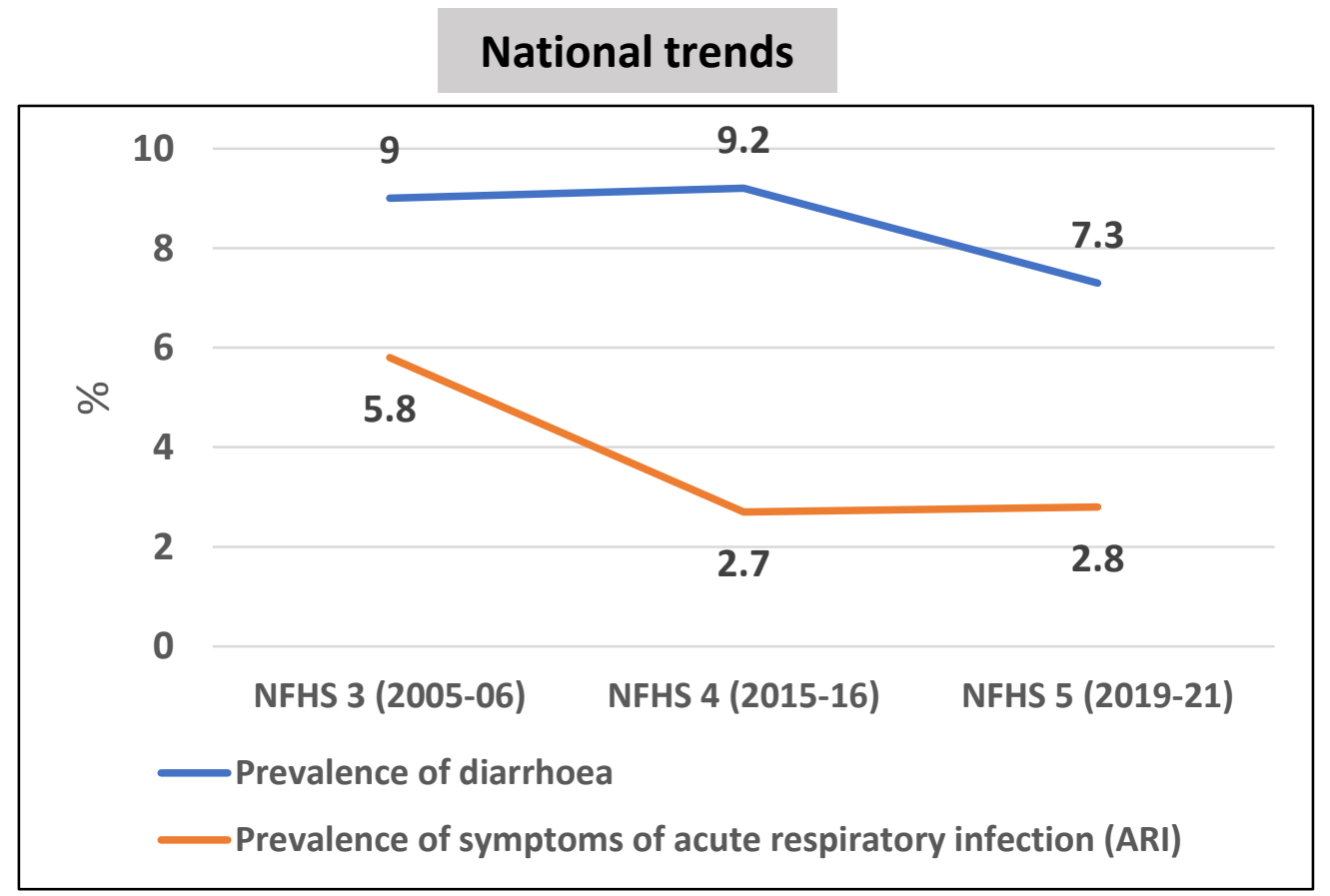

\begin{tabular}{|c|c|c|c|}
\hline Change over NFHS 4 & $\begin{array}{l}\text { Number } \\
\text { of States } \\
\text { improving }\end{array}$ & $\begin{array}{l}\text { Number of } \\
\text { States with } \\
\text { no change }\end{array}$ & $\begin{array}{l}\text { Number of } \\
\text { States } \\
\text { worsening }\end{array}$ \\
\hline Prevalence of diarrhoea & 23 & 0 & 13 \\
\hline $\begin{array}{l}\text { Prevalence of symptoms } \\
\text { of acute respiratory } \\
\text { infection (ARI) }\end{array}$ & 18 & 1 & 17 \\
\hline
\end{tabular}




\section{Childhood diseases}

Change in Prevalence of diarrhoea \& symptoms of acute respiratory infection (ARI) in the 2 weeks preceding the survey in India as per NFHS - 4\& 5 (2015-16 \& 2019-21)

\section{Prevalence of diarrhoea (-1.9\%)}

Prevalence of ARI (+0.1\%)

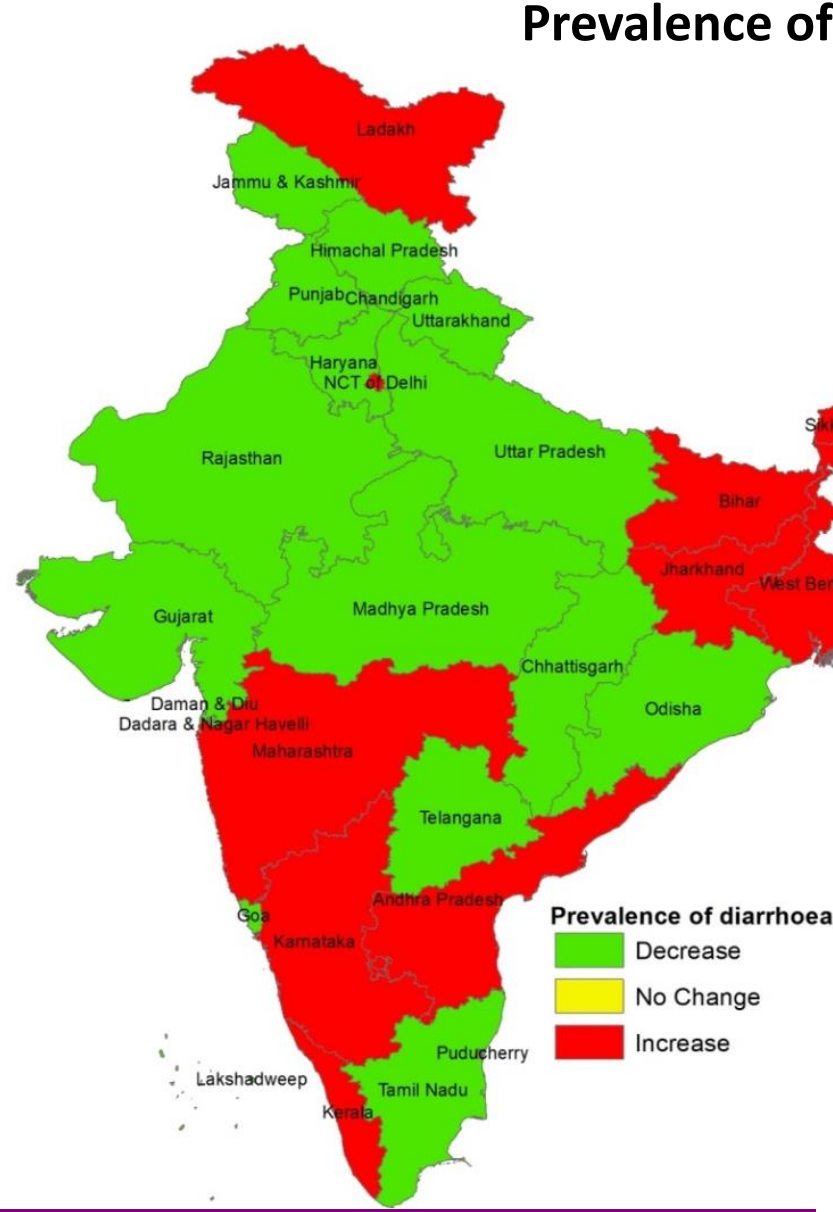

\section{Click here for State} wise change

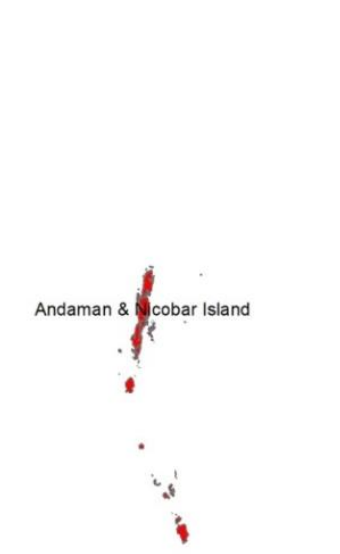

Uttarakhand(-12.6\%), Uttar Pradesh(-9.4\%), Puducherry(-7.6\%), Chhattisgarh (-5.5\%), Tamil Nadu(-4.3\%)
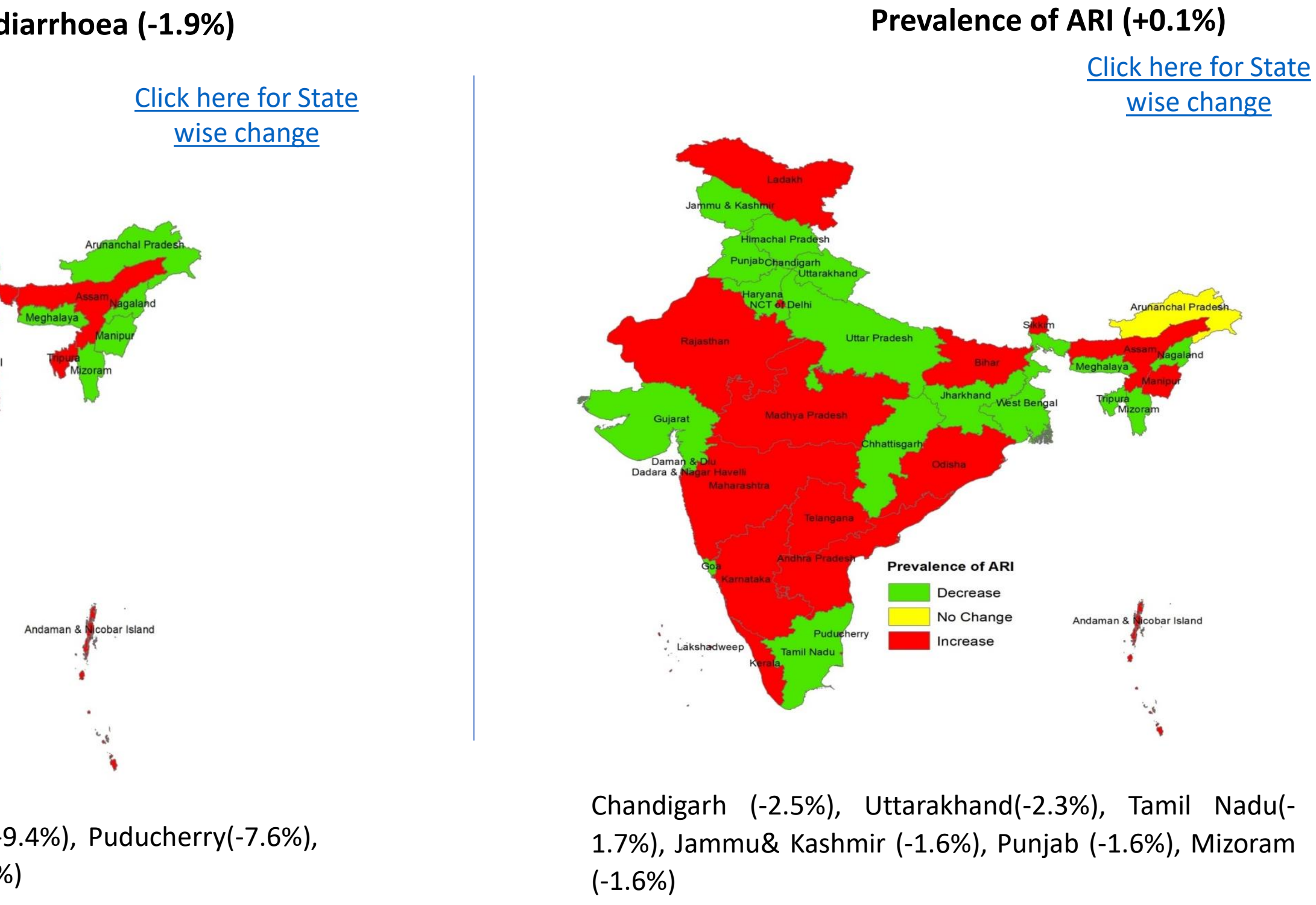


\section{Treatment of childhood diseases $\&$ vaccination}

- An increase was observed in the percentage of children with diarrhoea, who received ORS and Zinc, full immunization nationally.

- The percentage of children with symptoms of acute respiratory infection (ARI) who were taken to health facility has declined nationally.

\section{National trends}

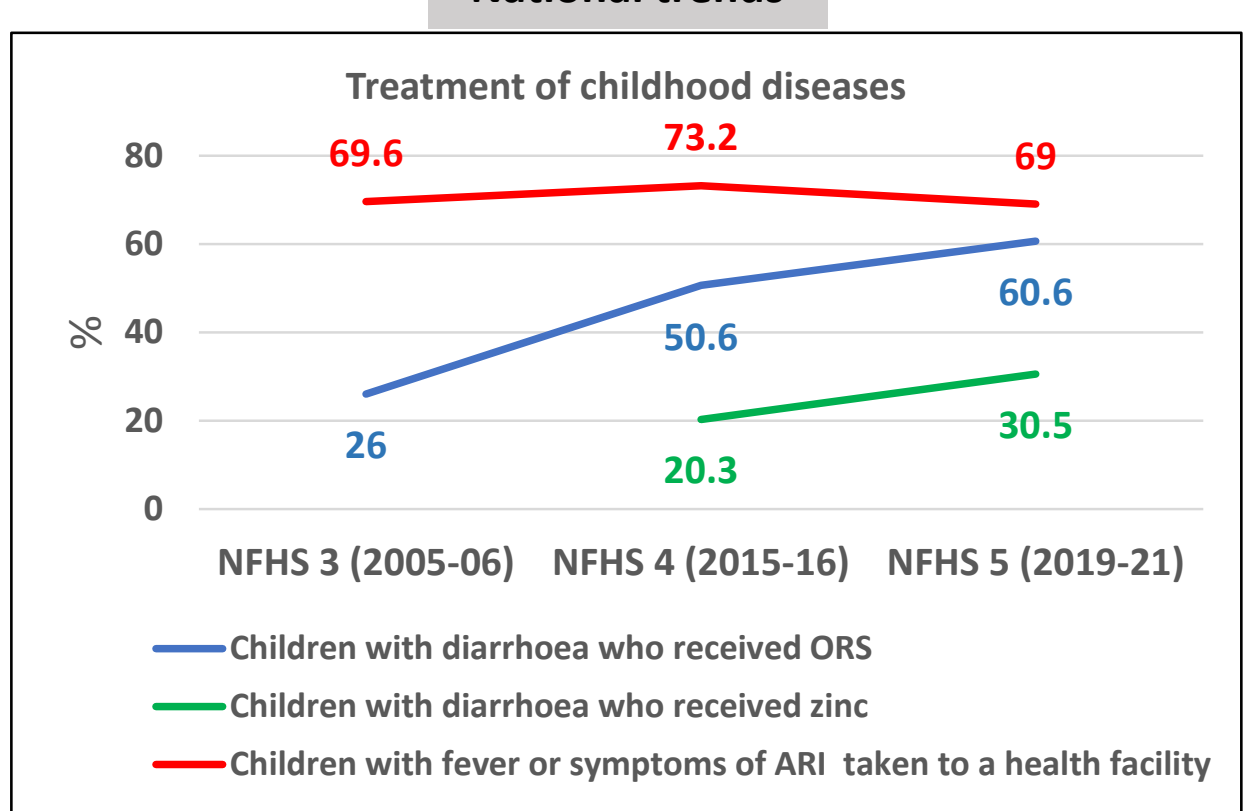

National trends

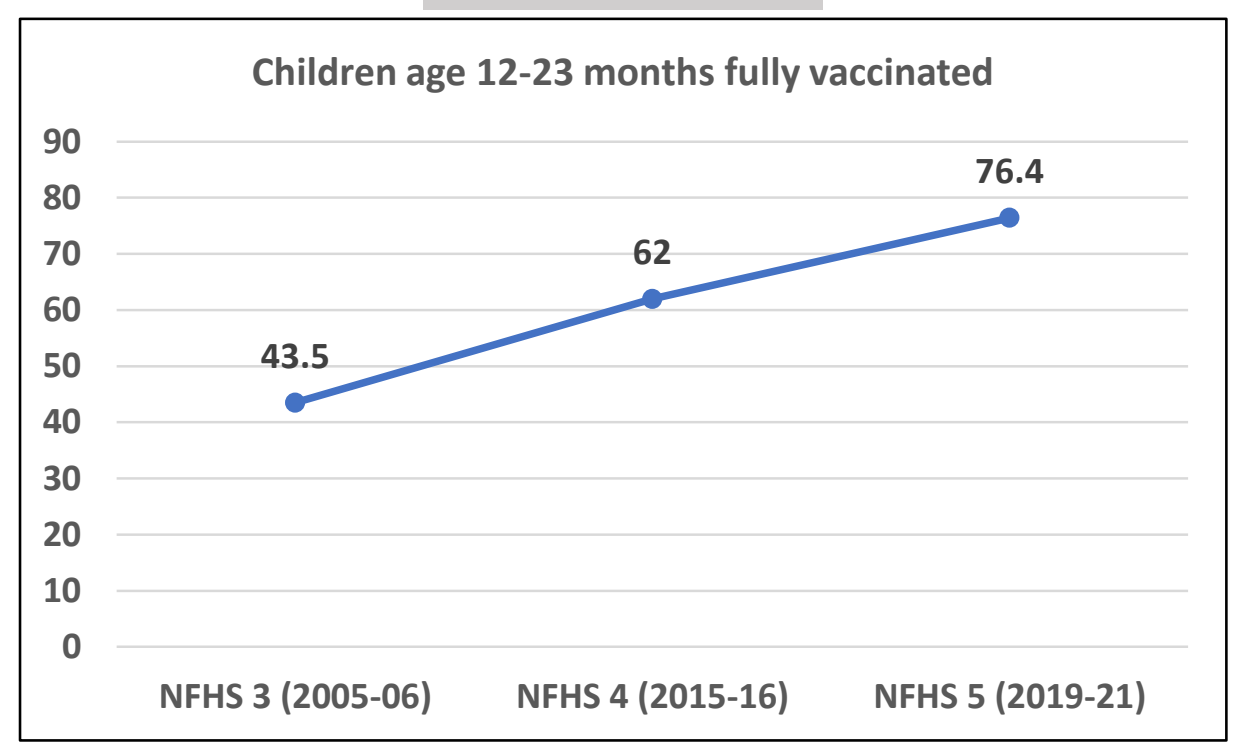

Click here for State findings

\begin{tabular}{|l|l|l|l|}
\hline Change over NFHS 4 & $\begin{array}{l}\text { Number of States } \\
\text { improving }\end{array}$ & $\begin{array}{l}\text { Number of States } \\
\text { with no change }\end{array}$ & $\begin{array}{l}\text { Number of States } \\
\text { worsening }\end{array}$ \\
\hline $\begin{array}{l}\text { Children with diarrhoea who } \\
\text { received ORS }\end{array}$ & 18 & 1 & 10 \\
\hline $\begin{array}{l}\text { Children with diarrhoea who } \\
\text { received zinc }\end{array}$ & 23 & 0 & 6 \\
\hline \begin{tabular}{l} 
Children with fever or symptoms \\
\hline ARI taken to a health facility
\end{tabular} & 7 & 0 & 27 \\
\hline Children fully immunized & 30 & 0 & 6 \\
\hline
\end{tabular}




\section{Treatment of childhood diseases $\&$ vaccination}

Change in Prevalence of Children with diarrhoea who received ORS and Zinc the survey in India as per NFHS $-4 \& 5$ (2015-16 \& 2019-21)

\section{Children with diarrhoea who received ORS (+10\%)}

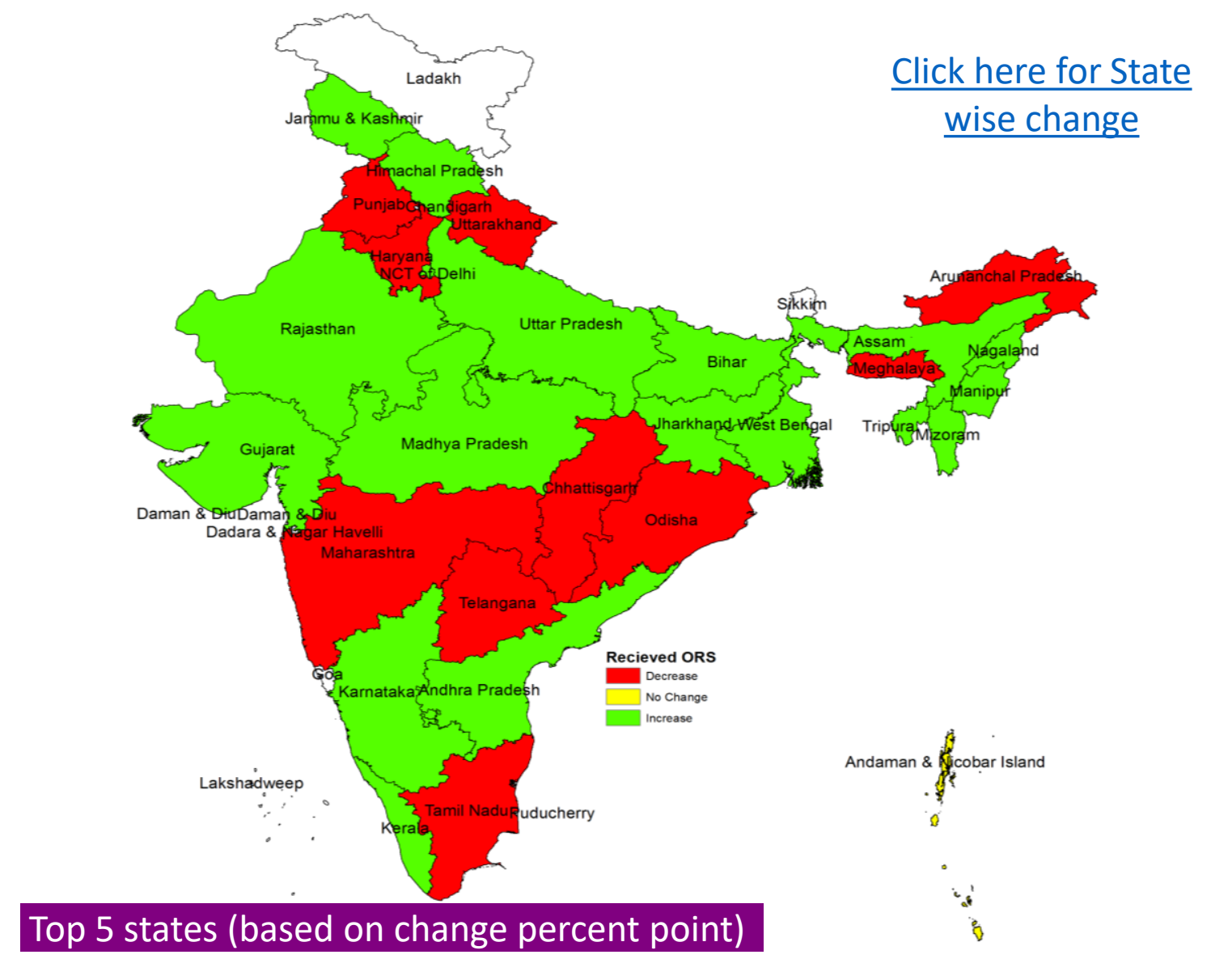

Tripura(+ 20.9), Gujarat(+ 20.3\%), Karnataka(+ 18.5\%), Assam(+ 17.2\%), Andhra Pradesh (+14.9\%)
Children with diarrhoea who received zinc $(+10.2 \%)$

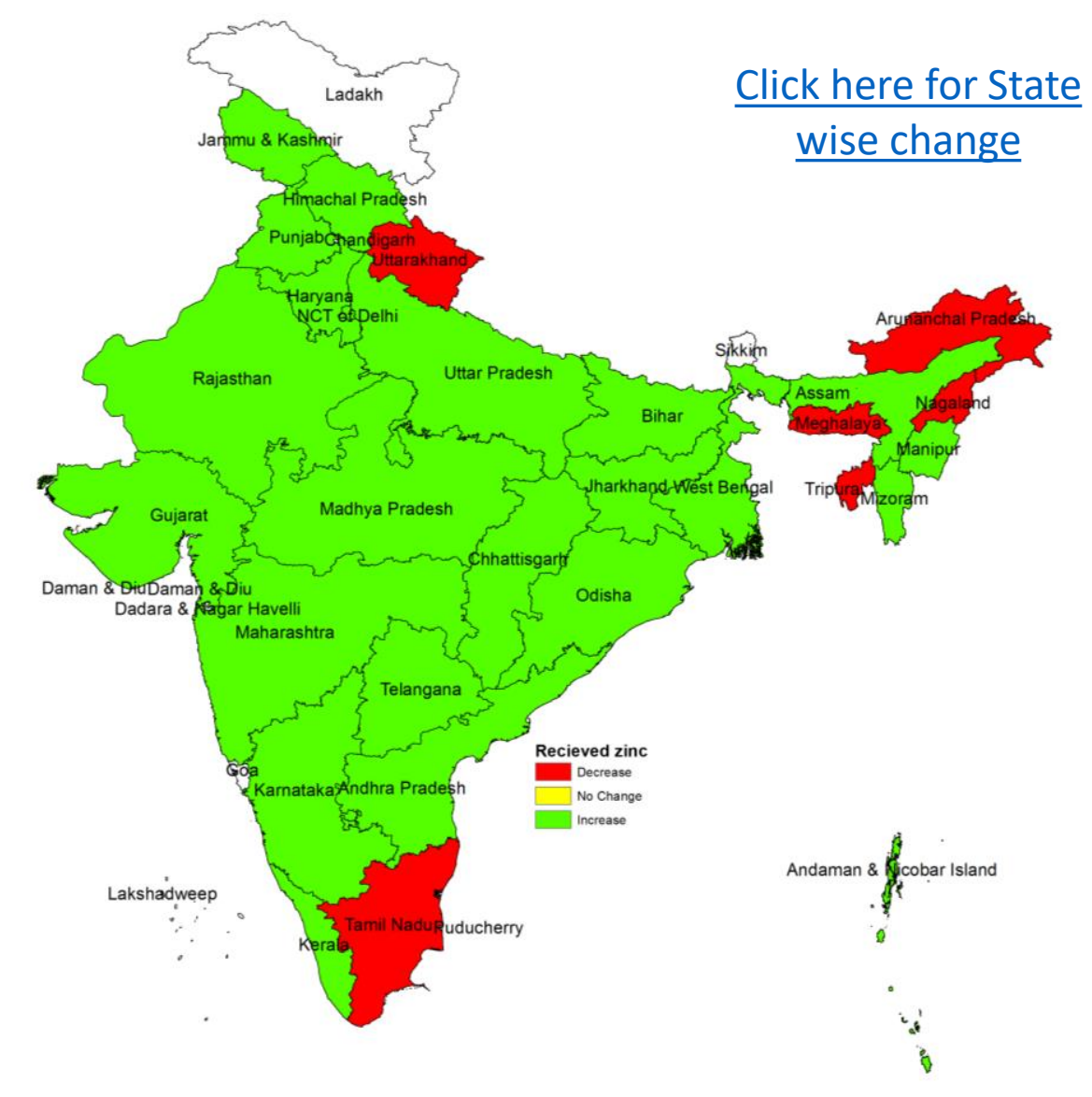

Andaman \&Nicobar (+ 35.8\%), Odisha(+ 19.9), Gujarat (+18.0\%), Uttar Pradesh (+15.9\%), West Bengal (+15.2\%) 
Change in Prevalence of Children with fever or symptoms of ARI taken and to a health facility and children age 12-23 months fully immunized in India as per NFHS - 4\& 5 (2015-16 \& 2019-21)

Children with fever or symptoms ARI taken to a health facility $(-4.2 \%)$

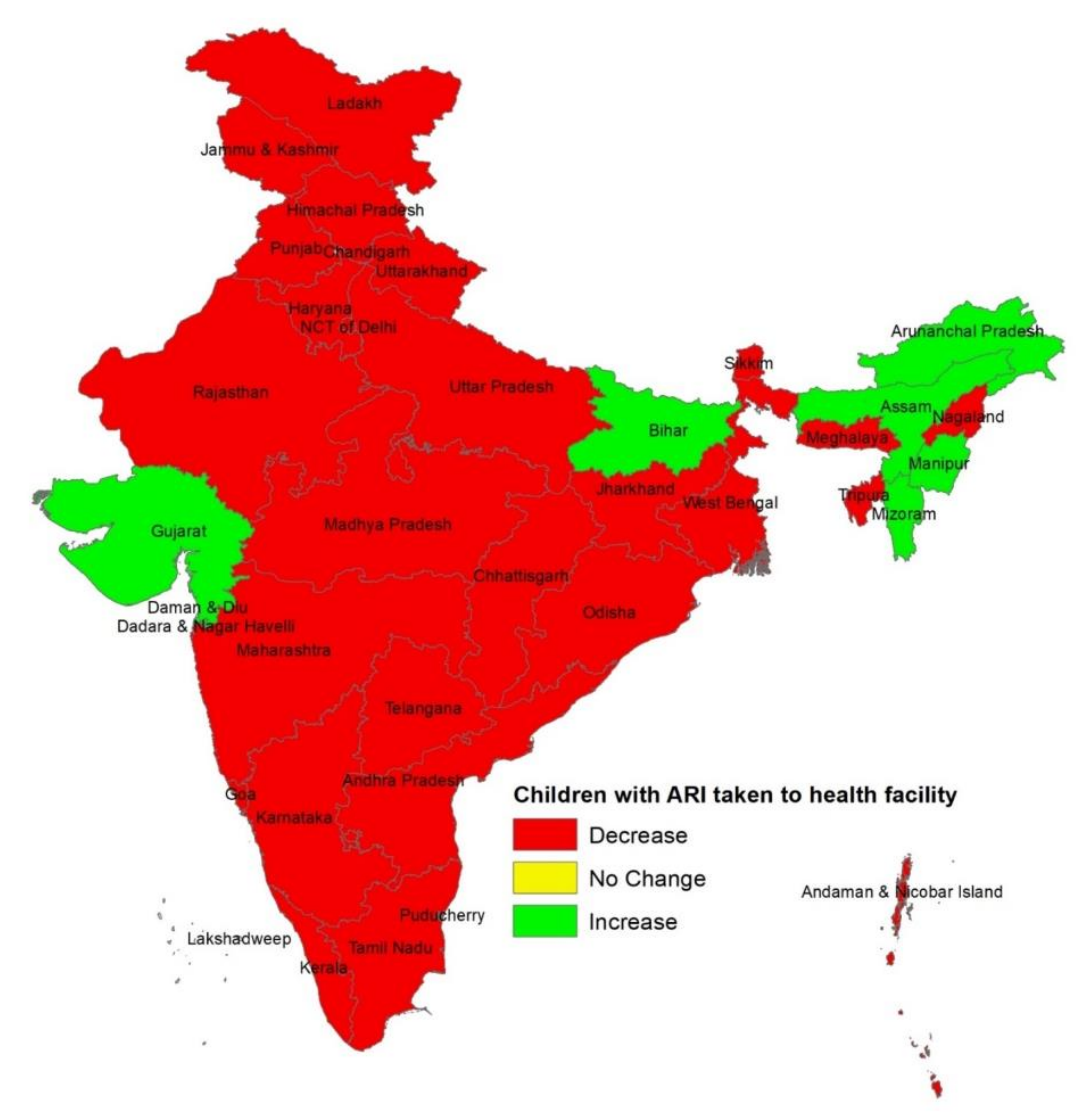

$\underline{\text { State findings }}$

Top 5 states (based on change percent point)

Dadar and Nagar haveli \& Daman and Diu (+13.2\%), Bihar (+9.6\%), Arunachal Pradesh(+9.6), Gujarat(5\%), Assam(+4.4\%), Mizoram(+2.9)
Children fully immunized (+ $14.4 \%)$

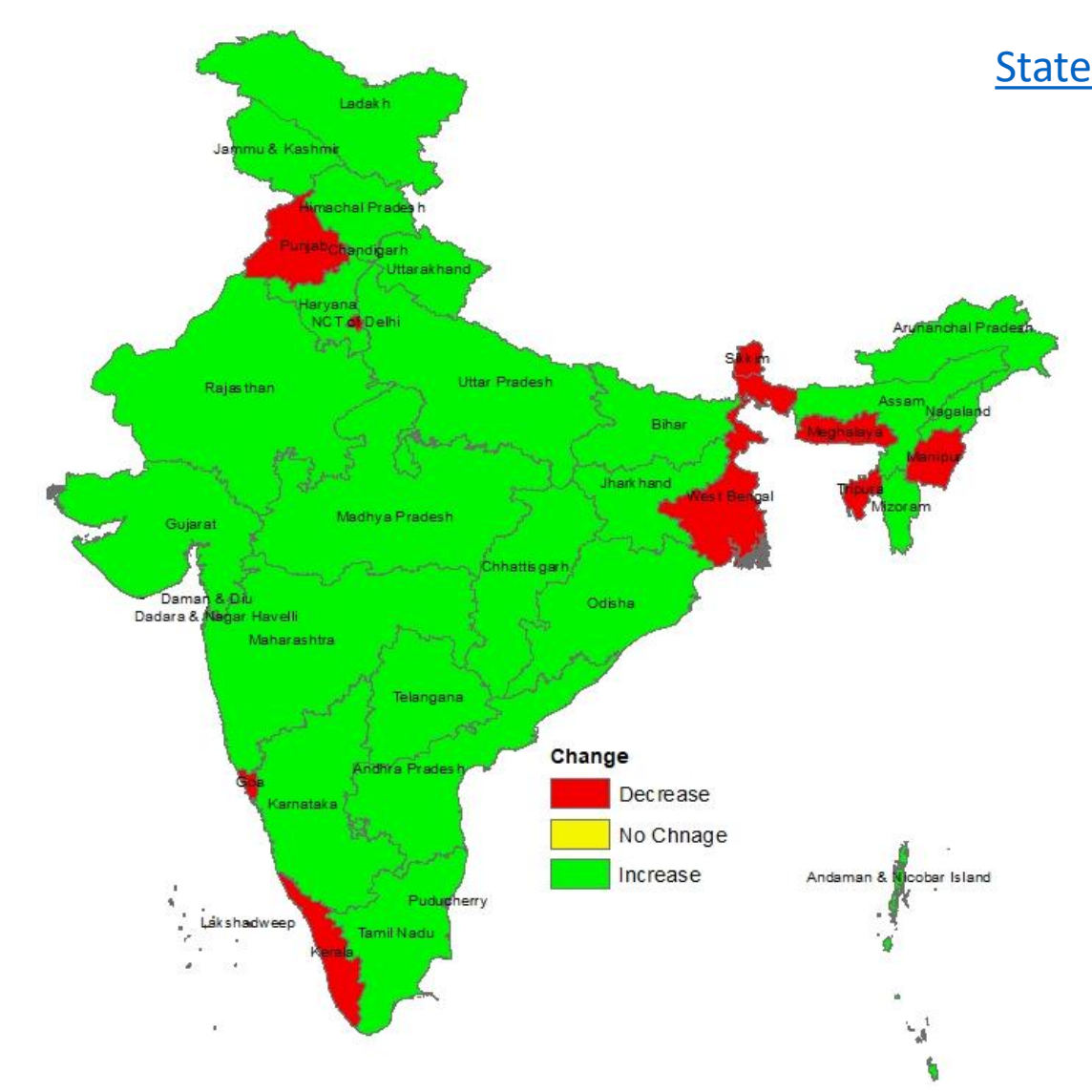

Dadra \& Nagar Haveli \& Daman \& Diu (+ 44.4\%), Arunachal Pradesh (+ $26.7 \%)$, Gujarat (+ 25.9\%), Rajasthan (+ 25.6\%), Madhya Pradesh (+ 23.5\%) 


\section{Child feeding practices}

- A secular increase was observed in early initiation of breastfeeding \& exclusive breastfeeding rates nationally.

- Complementary feeding practices have improved but the change is not satisfactory/substantial.
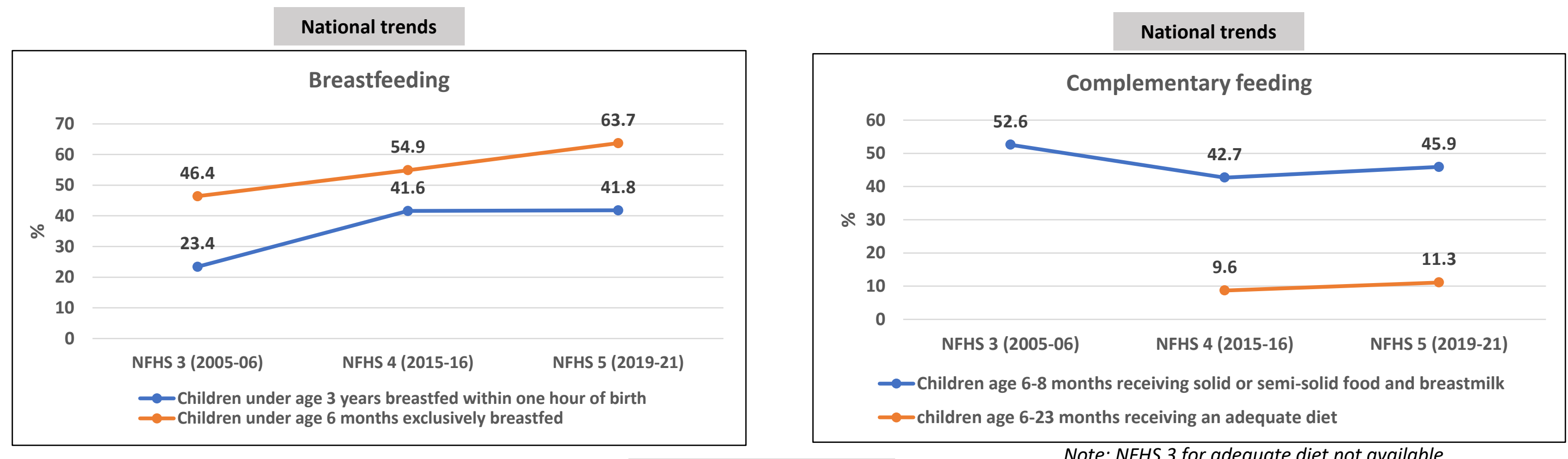

Click here for State findings

\begin{tabular}{|c|c|c|c|}
\hline Change over NFHS 4 for India & $\begin{array}{l}\text { Number of } \\
\text { States } \\
\text { improving }\end{array}$ & $\begin{array}{l}\text { Number of } \\
\text { States with no } \\
\text { change }\end{array}$ & $\begin{array}{l}\text { Number of States } \\
\text { worsening }\end{array}$ \\
\hline Children under age 3 years breastfed within one hour of birth & 17 & 1 & 18 \\
\hline Children under age 6 months exclusively breastfed & 28 & 0 & 6 \\
\hline Children age 6-8 months receiving solid or semi-solid food \& breastmilk & 16 & 0 & 15 \\
\hline Children age 6-23 months receiving an adequate diet & 28 & 0 & 8 \\
\hline
\end{tabular}




\section{Child feeding practices}

Change in Prevalence of children under age 3 years breastfed within one hour of birth and children under age 6 months exclusively breastfeed in India as per NFHS - 4\&5 (2015-16 \& 2019-21)

Breastfed within one hour of birth $(+0.2 \%)$

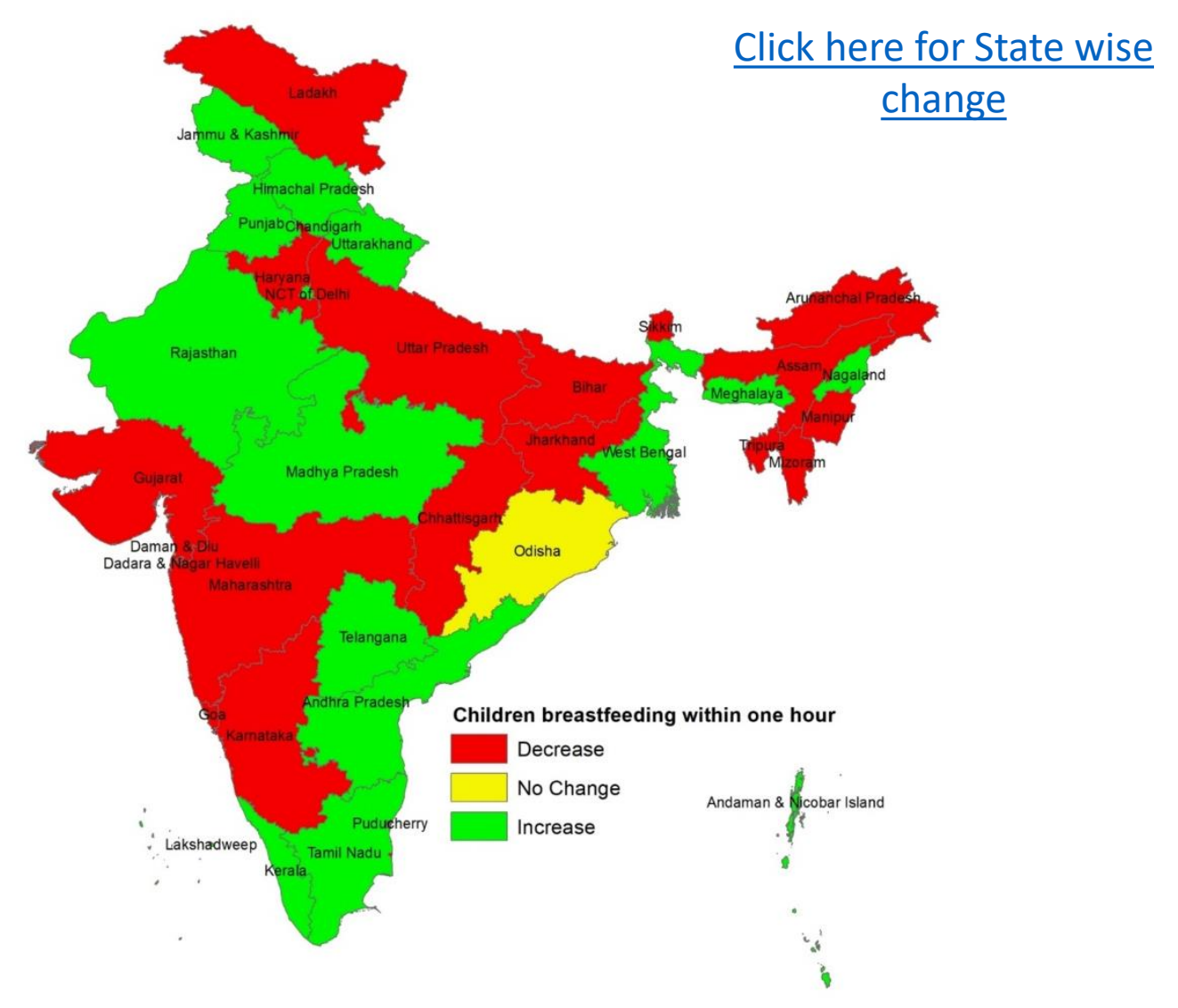

Top 5 states (based on change percent point)

Chandigarh (+30.2\%), NCT Delhi (23.2\%), Punjab

(+22.4\%), Lakshadweep (+18.6 \%), Meghalaya

$(+18.2 \%)$

\section{Exclusively breastfeed (+8.8\%)}

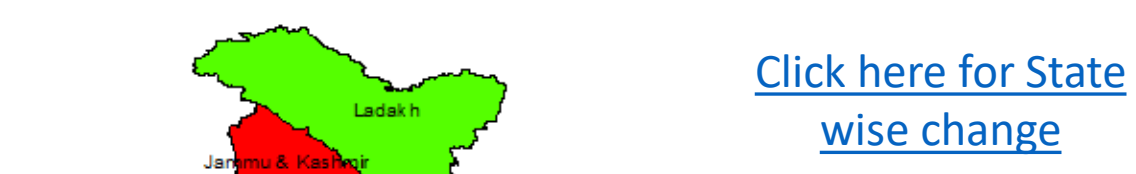

\footnotetext{
Puducherry (+19.3\%), Haryana $(+19.25 \%)$, Uttar Pradesh (+18.1\%), Madhya Pradesh(+15.8\%), NCT Delhi(+14.7\%)
} 


\section{Child feeding practices}

Change in prevalence of children under age 6-8 months receiving solid or semisolid food and breastmilk and total children age 6-23 months receiving an adequate diet in India as per NFHS - 4 \&5 (2015-16 \& 2019-21)

\section{Children receiving solid or semi solid food and} breastmilk (+3.2\%)

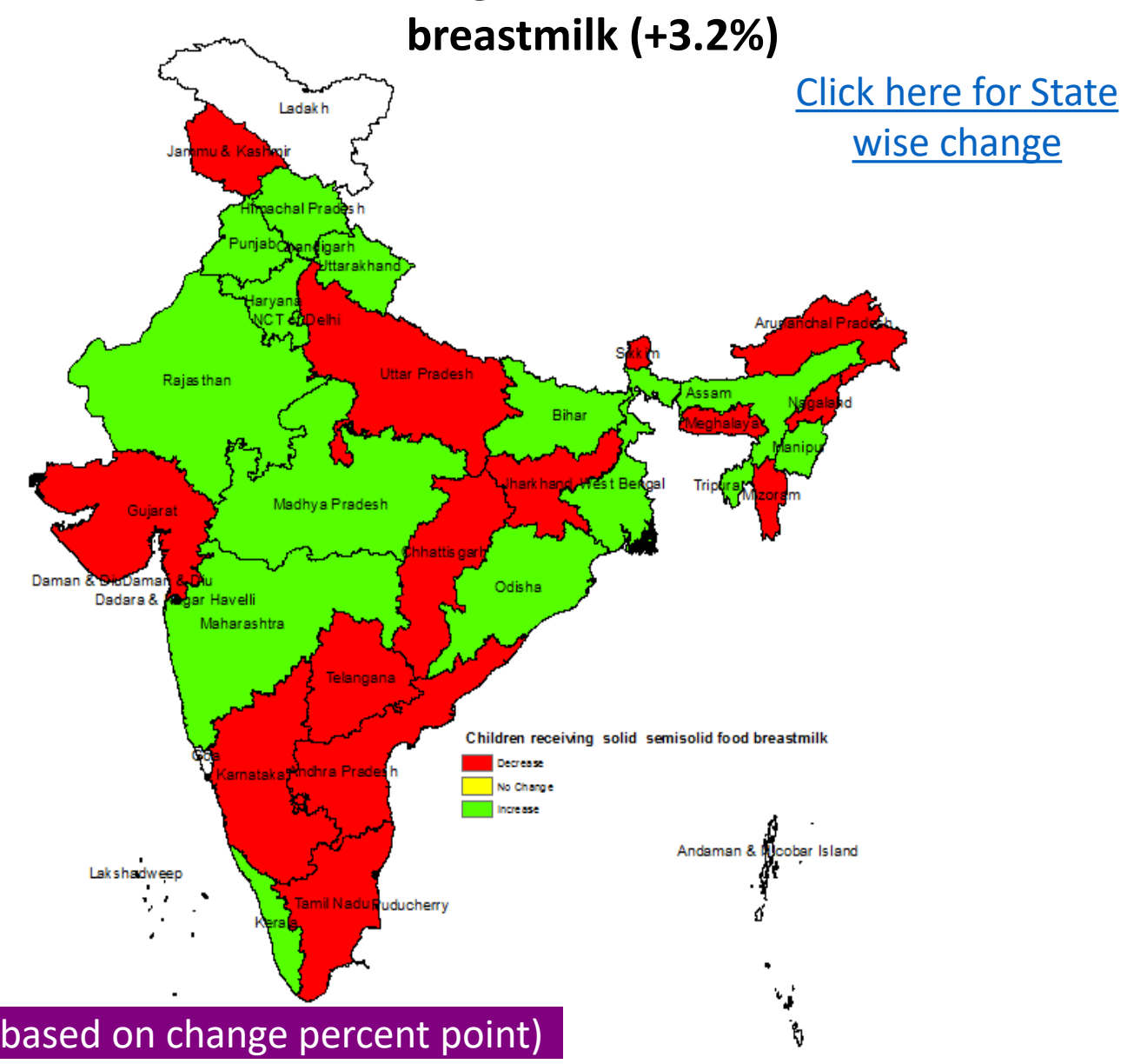

Tripura (+39.5\%), NCT Delhi $(+27.5 \%)$, Dadra \& Nagar Haveli \& Daman \& Diu (+23.7\%), West Bengal(+15.8\%), Himachal Pradesh (+15.\%)

\section{Children receiving Adequate diet (+1.7\%)}

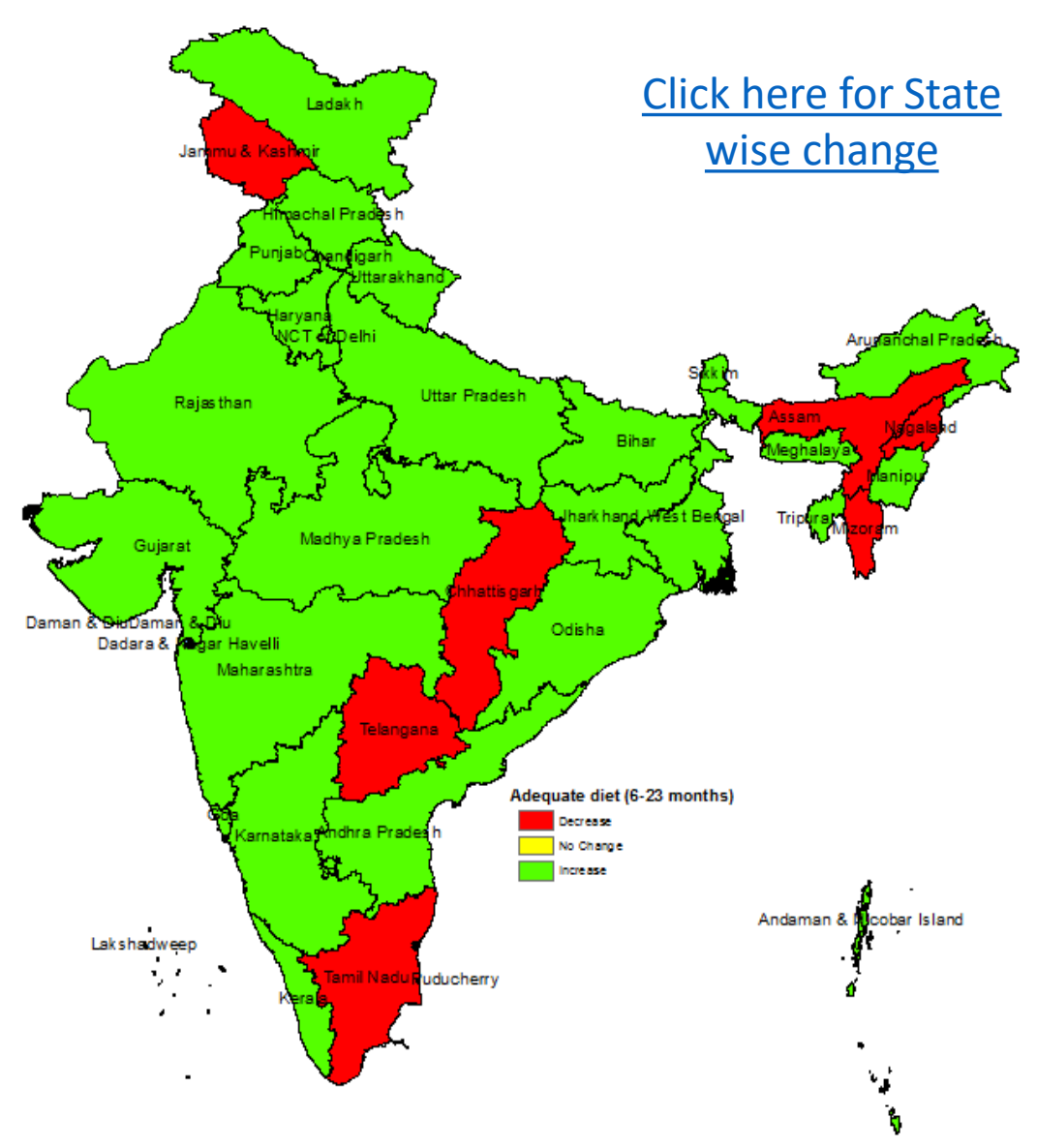

Chandigarh(+ 19\%), Odisha(+ 11.9\%) , NCT Delhi(+11.6\%), Goa(+11.1\%), Himachal Pradesh $(+8.1 \%)$ 


\section{Population and household profile-Household aspects}

A secular increase was observed in households with improved drinking water source, electricity and improved sanitation facility.

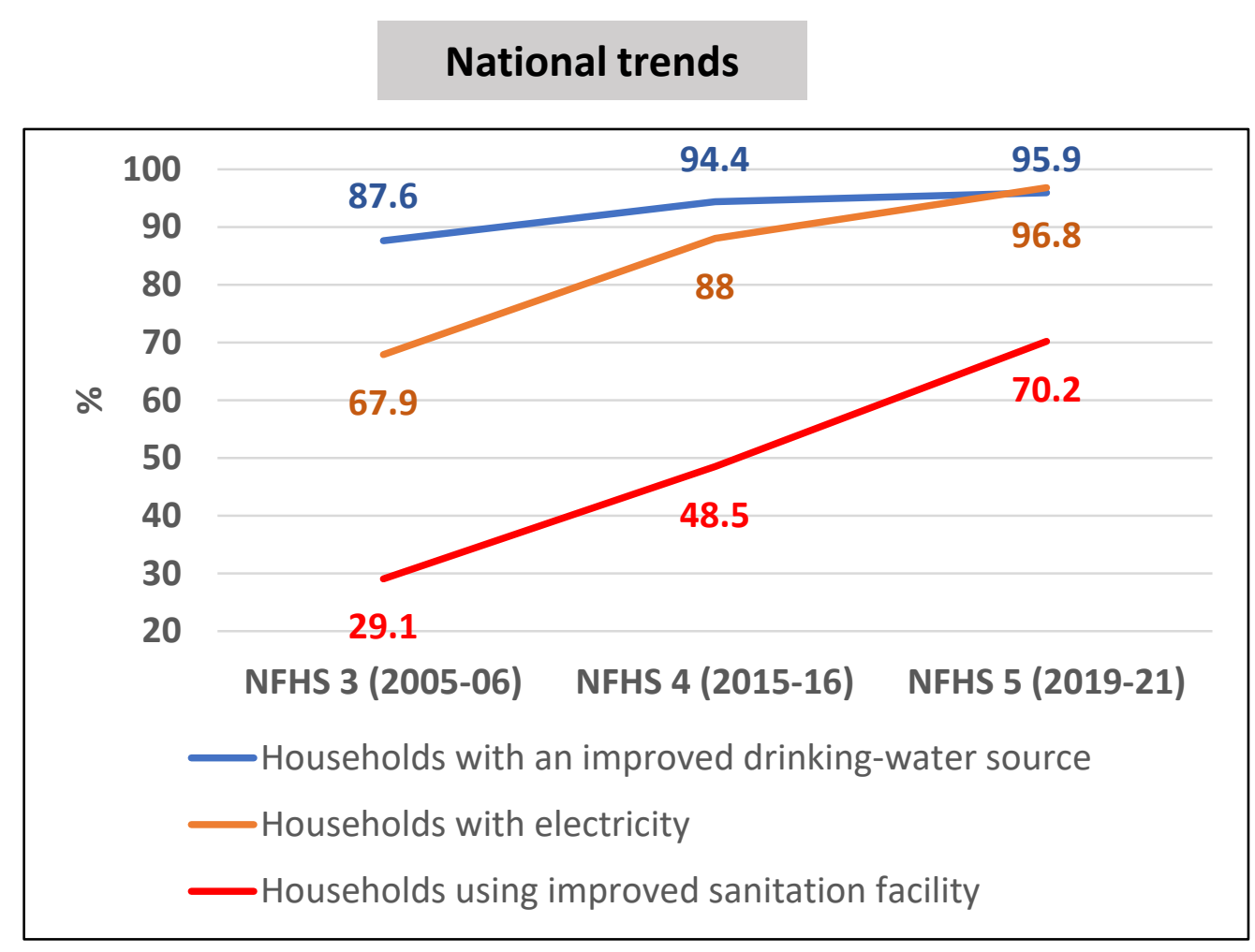

Click here for State findings
\begin{tabular}{|l|l|l|l|}
\hline $\begin{array}{l}\text { Change over } \\
\text { NFHS 4 }\end{array}$ & $\begin{array}{l}\text { Number of } \\
\text { States } \\
\text { improving }\end{array}$ & $\begin{array}{l}\text { Number of } \\
\text { States with } \\
\text { no change }\end{array}$ & $\begin{array}{l}\text { Number of } \\
\text { States } \\
\text { worsening }\end{array}$ \\
\hline \begin{tabular}{l} 
Households with \\
an improved \\
\hline drinking-water
\end{tabular} & 30 & 1 & 4 \\
\hline source & 33 & 1 & 2 \\
\hline $\begin{array}{l}\text { Households with } \\
\text { electricity }\end{array}$ & 33 & 1 & 1 \\
\hline $\begin{array}{l}\text { Households using } \\
\text { improved } \\
\text { sanitation facility }\end{array}$ & 35 & 0 & \\
\hline
\end{tabular}




\section{Population and household profile-Household aspects}

Change in prevalence of population living in households with an improved drinking water source, with electricity \& improved sanitation, India NFHS - 4 \&5 (2015-16 \& 2019-21)

Improved drinking water source(+1.5\%)

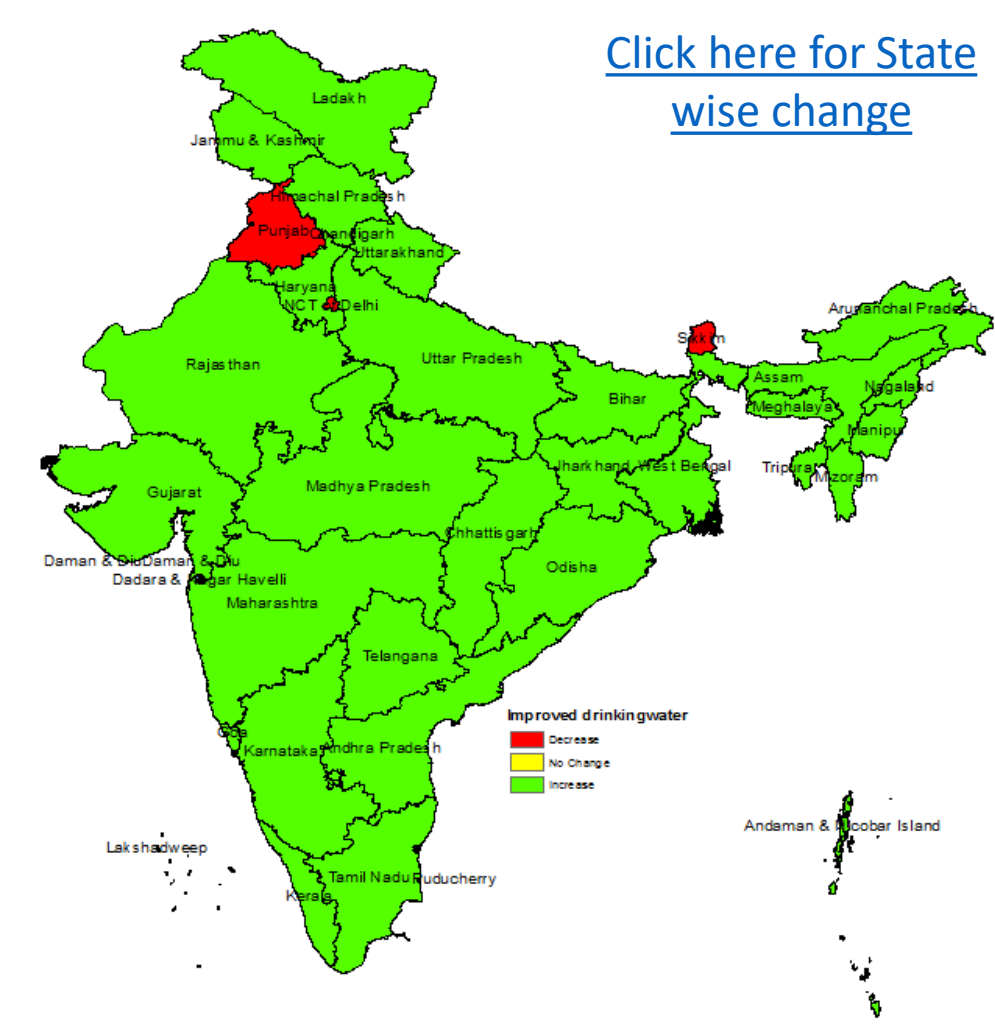

Top 5 states (based on change percent point)

Manipur(+13.2\%), Meghalaya (+8.9\%), Nagaland(+8.6\%), Jharkhand(+8.5\%), Arunachal Pradesh(+7.5\%) Back to contents

\section{Electricity $(+8.8 \%)$}

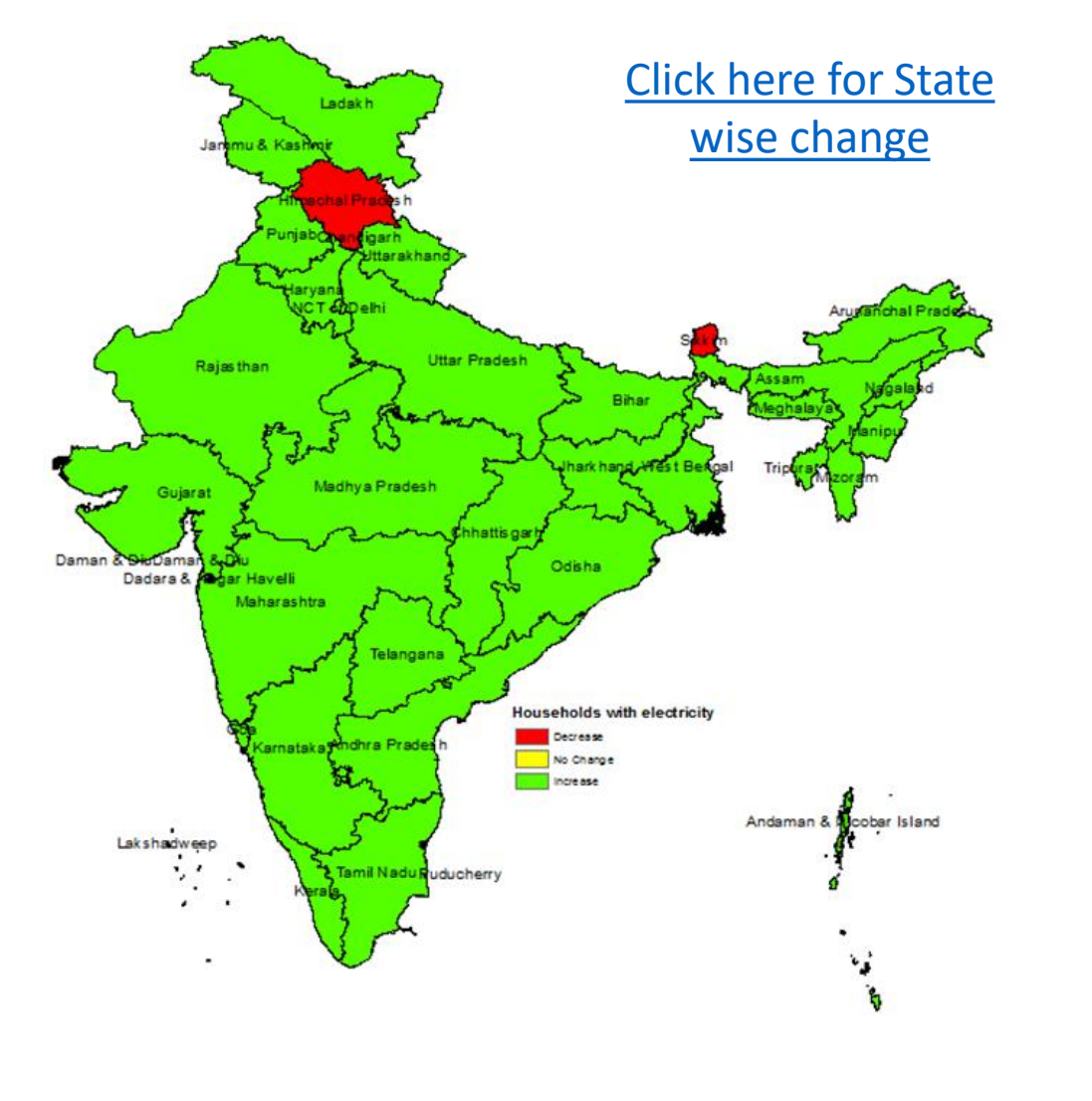

Bihar(+36.3\%), Uttar Pradesh

$(+18.4 \%)$,Assam(+14.3\%), Jharkhand(+13.1\%),

Odisha(+10.4\%)
Improved sanitation(+21.7)

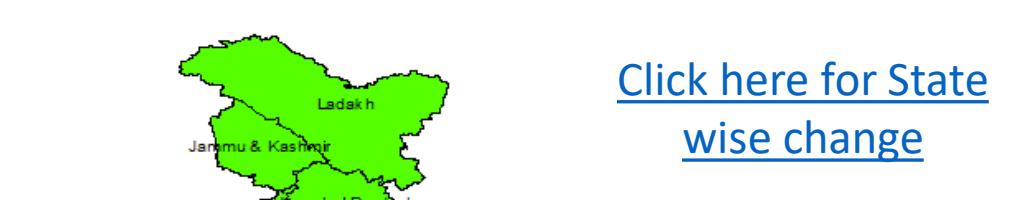

Chhattisgarh (+42\%), Uttar Pradesh (+32.4\%), Jharkhand(+31.7\%), Odisha(+30.5\%), Madhya Pradesh (+30.3\%) 


\section{Population and household profile-Gender aspects}

- A secular increase was observed in the sex ratio of the total population \& women with at least 10 years of schooling nationally

- Decline in the percentage of women who were married before the age of 18 years, nationally

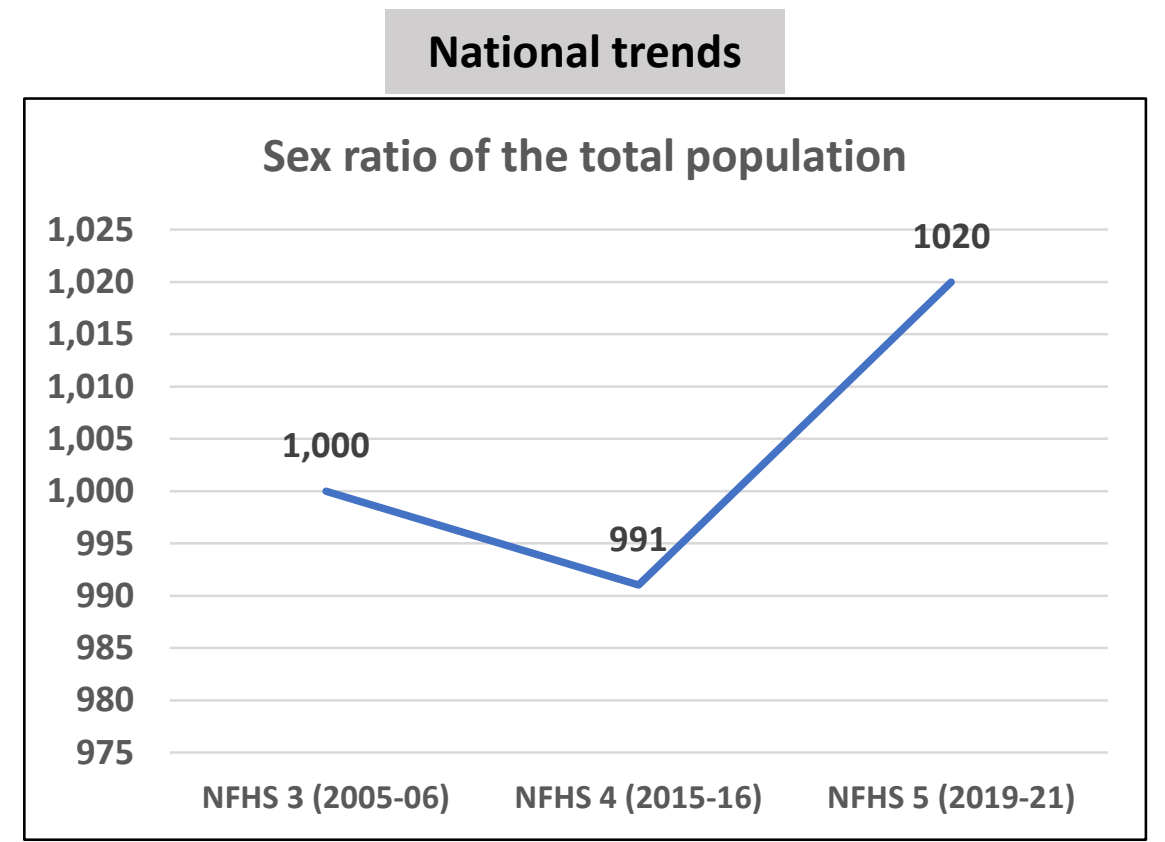

National trends

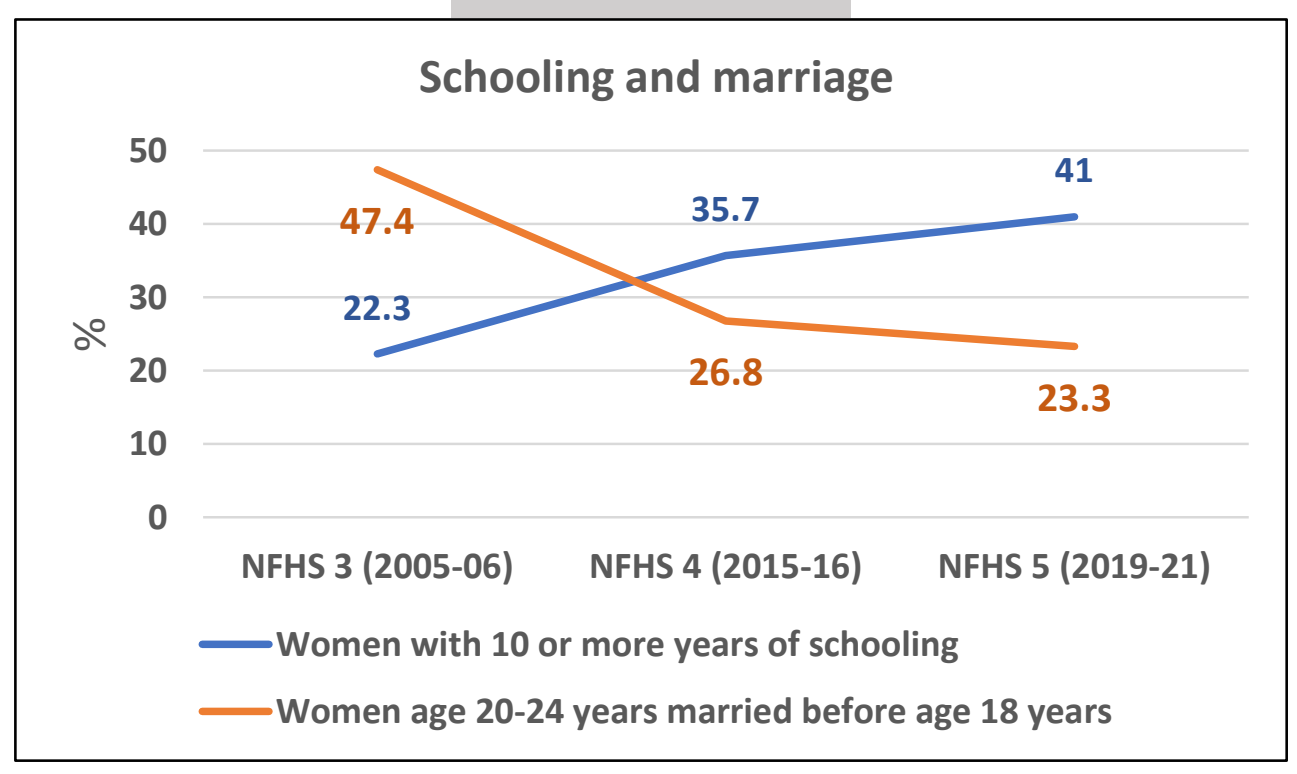

Click here for State findings

\begin{tabular}{|l|l|l|l|}
\hline Change over NFHS 4 & $\begin{array}{l}\text { Number of States } \\
\text { improving }\end{array}$ & $\begin{array}{l}\text { Number } \\
\text { of States } \\
\text { with no } \\
\text { change }\end{array}$ & $\begin{array}{l}\text { Number of } \\
\text { States } \\
\text { worsening }\end{array}$ \\
\hline $\begin{array}{l}\text { Sex ratio of the total } \\
\text { population }\end{array}$ & 30 & 0 & 6 \\
\hline $\begin{array}{l}\text { Women with 10 or more } \\
\text { years of schooling }\end{array}$ & 34 & 0 & 2 \\
\hline $\begin{array}{l}\text { Women age } 20 \text {-24 years } \\
\text { married before age } 18 \text { years }\end{array}$ & 30 & 2 & 4 \\
\hline \hline
\end{tabular}




\section{Population and household profile-Gender aspects}

Change in Prevalence of women with 10 or more years of schooling, Women age 20-24 years married before age 18 years, and sex ratio in India as per NFHS $-4 \& 5$ (2015-16 \& 2019-21)

\section{Sex ratio of the total population (+29)}

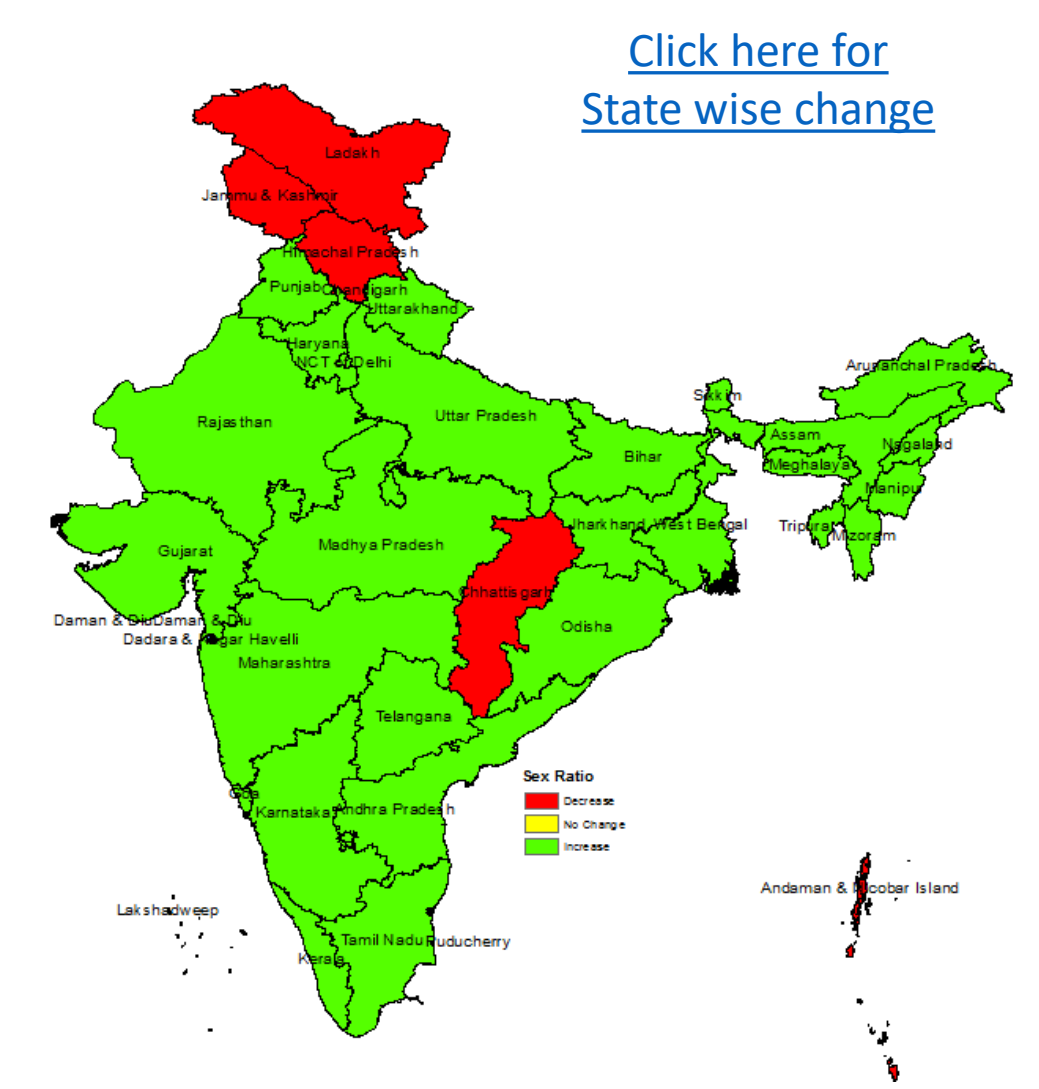

Top 5 states (based on change percent point)

Lakshadweep (+165), Kerala(+72), Puducherry $(+44)$, Bihar $(+28)$, Tamil Nadu $(+55)$
Women with 10 or more years of schooling $(+5.3 \%)$

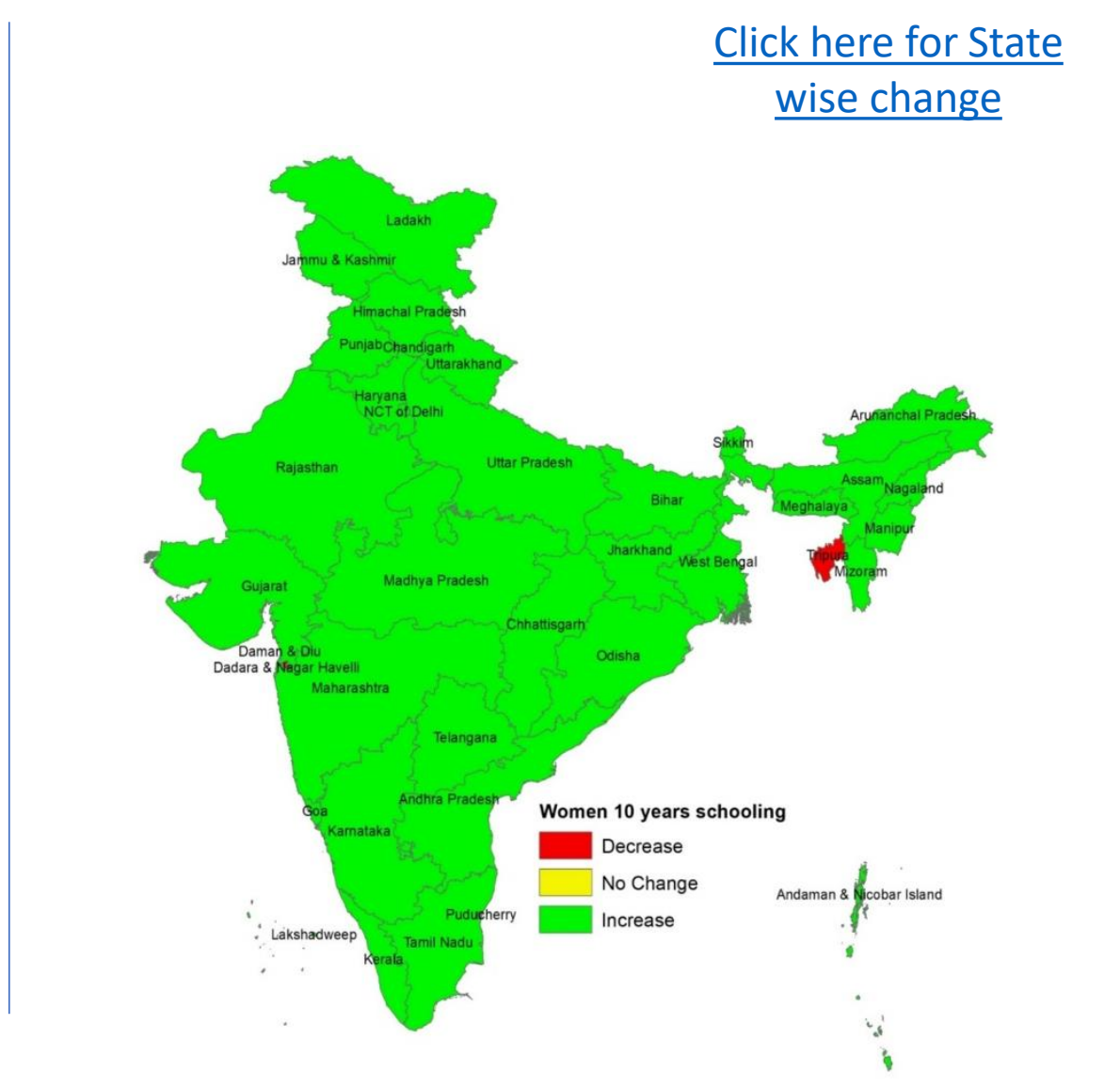

Jammu\&Kashmir(+14.2\%),Goa(13.3\%),Nagaland(+11.1 $\%)$, Lakshadweep(+11\%), Chhattisgarh (+10.4\%)
Women married before 18-years (-3.5\%)

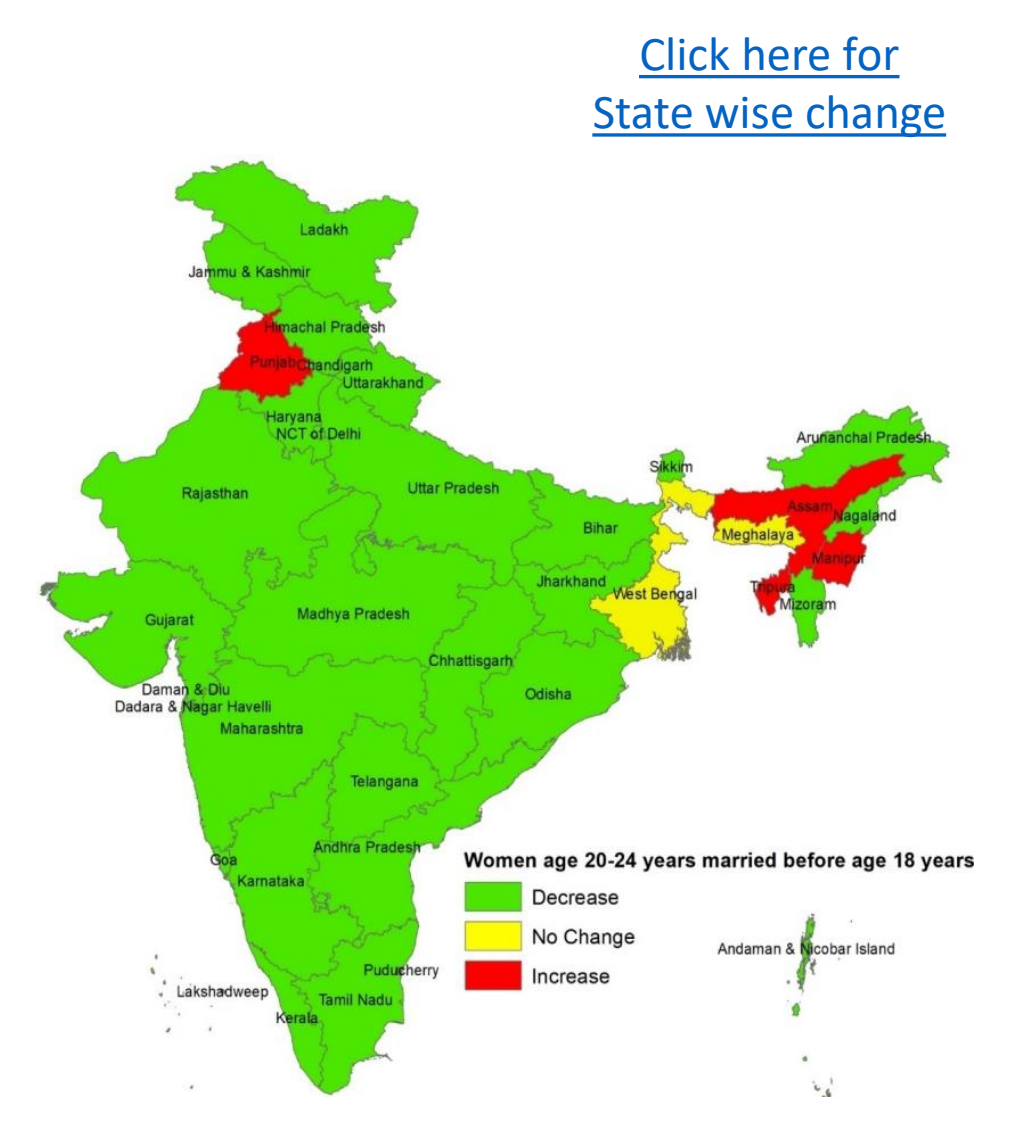

Rajasthan(-10\%), Madhya Pradesh (-9.3\%), Chhattisgarh (-9.2\%), Nagaland (-7.8\%), Haryana $(-6.9 \%)$ 


\title{
ANNEXURES
}

STATE FINDINGS-

\author{
Snapshots
}




\section{Child Mortality Rate}

SDG 2030 target for Neonatal mortality rate: $<12$

SDG target Achieved

SDG target Not Achieved

Improvement in the indicator

Decline in the indicator

No change

States arranged as per lowest to highest prevalence (NFHS-5)

Data for Lakshadweep, Goa (NFHS 4 \& 5) and Andaman \& Nicobar Islands (NFHS 4) is based on 250499 unweighted person-years of exposure to the risk of death

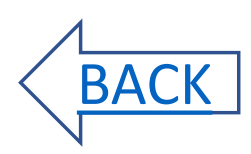

\begin{tabular}{|c|c|c|c|}
\hline [StatedUts])Indicators & $\begin{array}{l}\text { Neona } \\
\text { (N) }\end{array}$ & tal mortalit & $\begin{array}{l}\text { ity rate } \\
\text { tS } 5\end{array}$ \\
\hline RDUND & NFHS 4 & NFHS 5 & CHANGE \\
\hline INDIA & 29.5 & 24.9 & -4.6 \\
\hline Lakshadweep & 23.3 & 0.0 & -23.3 \\
\hline Puducherry & 5.8 & 2.3 & -3.5 \\
\hline Kerala & 4.4 & 3.4 & -1.0 \\
\hline Sikkim & 20.8 & 5.0 & -15.8 \\
\hline Goa & 12.9 & 5.6 & -7.3 \\
\hline Arunachal Pradesh & 11.8 & 7.7 & -4.1 \\
\hline Jammu and Kashmir & 23.1 & 9.8 & -13.3 \\
\hline Nagaland & 16.5 & 10.2 & -6.3 \\
\hline Ladakh & 25.7 & 11.4 & -14.3 \\
\hline Mizoram & 11.2 & 11.4 & 0.2 \\
\hline Andaman \& Nicobar & 7.3 & 12.3 & 5.0 \\
\hline Tamil Nadu & 14.0 & 12.7 & -1.3 \\
\hline West bengal & 22.0 & 15.5 & -6.5 \\
\hline Karnataka & 18.5 & 15.8 & -2.7 \\
\hline Maharastra & 16.2 & 16.5 & 0.3 \\
\hline Telangana & 20.0 & 16.8 & -3.2 \\
\hline Manipur & 15.6 & 17.2 & 1.6 \\
\hline NCT Delhi & 17.8 & 17.5 & -0.3 \\
\hline Meghalaya & 18.3 & 19.8 & 1.5 \\
\hline Andhra pradesh & 23.6 & 19.9 & -3.7 \\
\hline Rajasthan & 29.8 & 20.2 & -9.6 \\
\hline Himachal pradesh & 25.5 & 20.5 & -5.0 \\
\hline Dadar\& nagar haveli and Daman \& Diu & 13.9 & 21.4 & 7.5 \\
\hline Haryana & 22.1 & 21.6 & -0.5 \\
\hline Gujarat & 26.8 & 21.8 & -5.0 \\
\hline Punjab & 21.2 & 21.8 & 0.6 \\
\hline Assam & 32.8 & 22.5 & -10.3 \\
\hline Tripura & 13.2 & 22.9 & 9.7 \\
\hline Odisha & 28.2 & 27.0 & -1.2 \\
\hline Jharkhand & 33.0 & 28.2 & -4.8 \\
\hline Madhya Pradesh & 36.9 & 29.0 & -7.9 \\
\hline Chattisgarh & 42.1 & 32.4 & -9.7 \\
\hline Uttarakhand & 27.9 & 32.4 & 4.5 \\
\hline BIHAR & 36.7 & 34.5 & -2.2 \\
\hline Uttar Pradesh & 45.1 & 35.70 & -9.4 \\
\hline Chandigarh & $\mathrm{na}$ & $\mathrm{na}$ & na \\
\hline
\end{tabular}

Infant mortality rate

\begin{tabular}{|c|c|c|c|}
\hline [Statetulsindicators] & & $\begin{array}{l}\text { it mortality } \\
\text { IRI)_NFHS }\end{array}$ & $\begin{array}{l}\text { rate } \\
55\end{array}$ \\
\hline ROUIND & NFHS 4 & NFHS 5 & CHANG \\
\hline INDIA & 40.7 & 35.2 & -5 \\
\hline Lakshadweep & 27.0 & 0.0 & -27 \\
\hline Puducherry & 15.7 & 2.9 & -12 \\
\hline Kerala & 5.6 & 4.4 & -1 \\
\hline Goa & 12.9 & 5.6 & -7 \\
\hline Sikkim & 29.5 & 11.2 & -18 \\
\hline Arunachal Pradesh & 22.9 & 12.9 & -10 \\
\hline Jammu and Kashmir & 32.4 & 16.3 & -16 \\
\hline Tamil Nadu & 20.2 & 18.6 & -1 \\
\hline Ladakh & 35.3 & 20.0 & -15 \\
\hline Andaman \& Nicobar & 9.8 & 20.6 & \\
\hline Mizoram & 40.1 & 21.3 & -18 \\
\hline West bengal & 27.5 & 22.0 & -5 \\
\hline Maharastra & 23.7 & 23.2 & 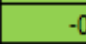 \\
\hline Nagaland & 29.5 & 23.4 & -6 \\
\hline NCT Delhi & 31.2 & 24.5 & -6 \\
\hline Manipur & 21.7 & 25.0 & \\
\hline Karnataka & 26.9 & 25.4 & \\
\hline Himachal pradesh & 34.3 & 25.6 & \\
\hline Telangana & 27.7 & 26.4 & \\
\hline Punjab & 29.2 & 28.0 & \\
\hline Andhra pradesh & 34.9 & 30.3 & -4 \\
\hline Rajasthan & 41.3 & 30.3 & -11 \\
\hline Gujarat & 34.2 & 31.2 & -5 \\
\hline Dadar\& nagar haveli and Daman \& Diu & 33.4 & 31.8 & -1 \\
\hline Assam & 47.6 & 31.9 & -15 \\
\hline Meghalaya & 29.9 & 32.3 & \\
\hline Haryana & 32.8 & 33.3 & \\
\hline Odisha & 39.6 & 36.3 & -3 \\
\hline Tripura & 26.7 & 37.6 & \\
\hline Jharkhand & 43.8 & 37.9 & -5 \\
\hline Uttarakhand & 39.7 & 39.1 & -6 \\
\hline Madhya Pradesh & 51.2 & 41.3 & 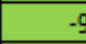 \\
\hline Chattisgarh & 54.0 & 44.3 & -9 \\
\hline BHAR & 48.1 & 46.8 & \\
\hline Uttar Pradesh & 63.5 & 50.4 & -13 \\
\hline Chandigarh & na & $\mathrm{na}$ & \\
\hline
\end{tabular}

SDG 2030 target for Under-five mortality rate: $\leq \mathbf{2 5}$

\begin{tabular}{|c|c|c|c|}
\hline [StatedUtsindicators] & $\begin{array}{l}\text { Under } \\
\text { (UP }\end{array}$ & $\begin{array}{l}\text {-five morta } \\
\text { GMMII)_NFH }\end{array}$ & $\begin{array}{l}\text { ality rate } \\
155\end{array}$ \\
\hline ROUNND & NFHS4 & NFHS5 & CHANGE \\
\hline INDIA & 49.7 & 41.9 & $\begin{aligned}-7.8 \\
\end{aligned}$ \\
\hline Lakshadweep & 30.2 & 0.0 & -30.2 \\
\hline Puducherry & 16.2 & 3.9 & -12.3 \\
\hline Kerala & 7.1 & 5.2 & -1.9 \\
\hline Goa & 12.9 & 10.6 & -2.3 \\
\hline Sikkim & 32.2 & 11.2 & -21.0 \\
\hline Jammu and Kashmir & 37.6 & 18.5 & -19.1 \\
\hline Arunachal Pradesh & 32.9 & 18.8 & -14.1 \\
\hline Tamil Nadu & 26.8 & 22.3 & -4.5 \\
\hline Mizoram & 46.0 & 24.0 & -22.0 \\
\hline Andaman \& Nicobar & 13.0 & 24.5 & 11.5 \\
\hline West bengal & 31.8 & 25.4 & -6.4 \\
\hline Maharastra & 28.7 & 28.0 & -0.7 \\
\hline Himachal pradesh & 37.6 & 28.9 & -8.7 \\
\hline Telangana & 31.7 & 29.4 & -2.3 \\
\hline Karnataka & 31.5 & 29.5 & -2.0 \\
\hline Ladakh & 40.6 & 29.5 & -11.1 \\
\hline Manipur & 25.9 & 30.0 & 4.1 \\
\hline NCT Delhi & 42.2 & 30.6 & -11.6 \\
\hline Puniab & 33.2 & 32.7 & -0.5 \\
\hline Nagaland & 37.5 & 33.0 & -4.5 \\
\hline Andhra pradesh & 40.8 & 35.2 & -5.6 \\
\hline Dadar\& nagar haveli and Daman \& Diu & 39.9 & 37.0 & -2.9 \\
\hline Gujarat & 43.5 & 37.6 & -5.9 \\
\hline Rajasthan & 50.7 & 37.6 & -13.1 \\
\hline Haryana & 41.1 & 38.7 & -2.4 \\
\hline Assam & 56.5 & 39.1 & -17.4 \\
\hline Meghalaya & 39.6 & 40.0 & 0.4 \\
\hline Odisha & 48.1 & 41.1 & -7.0 \\
\hline Tripura & 32.7 & 43.3 & 10.6 \\
\hline Jharkhand & 54.3 & 45.4 & -8.9 \\
\hline Uttarakhand & 46.5 & 45.6 & -0.9 \\
\hline Madhya Pradesh & 64.6 & 49.2 & -15.4 \\
\hline Chattisgarh & 64.3 & 50.4 & -13.9 \\
\hline BiHAR & 58.1 & 56.4 & -1.7 \\
\hline Uttar Pradesh & 78.1 & 59.8 & -18.3 \\
\hline Chandigarh & na & na & na \\
\hline
\end{tabular}




\section{Child Nutritional status}

SDG target Achieved

SDG target Not Achieved

Improvement in the indicator

Decline in the indicator

No change

States arranged as per lowest to highest prevalence (NFHS-5)

$$
\langle\overline{B A C K} \backslash \underline{N E X T}\rangle
$$

SDG 2030 target for wasting for children less than 3 year: $<3 \%$

\begin{tabular}{|c|c|c|c|}
\hline (States/Uts)/Indicators) & $\begin{array}{l}\text { Children u } \\
\text { stunted (he }\end{array}$ & $\begin{array}{l}\text { under } 5 \text { ye } \\
\text { eeight-for- }\end{array}$ & $\begin{array}{l}\text { ars who are } \\
\text { age)18 (\%) }\end{array}$ \\
\hline Round & NFHS4 & NFHS5 & CHANGE \\
\hline INDIA & 38.4 & 35.5 & -2.9 \\
\hline $\begin{array}{l}\text { Puducherry } \\
\end{array}$ & 23.7 & 20.0 & -3.7 \\
\hline Sikkim & 29.6 & 22.3 & -7.3 \\
\hline Andaman \& Nicobar Islands & 23.3 & 22.5 & -0.8 \\
\hline Kerala & 19.7 & 23.4 & 3.7 \\
\hline Manipur & 28.9 & 23.4 & -5.5 \\
\hline Punjab & 25.7 & 24.5 & -1.2 \\
\hline Tamil Nadu & 27.1 & 25.0 & -2.1 \\
\hline Chandigarh & 28.7 & 25.3 & -3.4 \\
\hline Goa & 20.1 & 25.8 & 5.7 \\
\hline Jammu \& Kashmir & 27.4 & 26.9 & -0.5 \\
\hline Uttarakhand & 33.5 & 27.0 & -6.5 \\
\hline Haryana & 34.0 & 27.5 & -6.5 \\
\hline Arunachal Pradesh & 29.4 & 28.0 & -1.4 \\
\hline Mizoram & 28.1 & 28.9 & 0.8 \\
\hline Ladakh & 30.9 & 30.5 & -0.4 \\
\hline Himachal Pradesh & 26.3 & 30.8 & 4.5 \\
\hline NCT Delhi & 31.9 & 30.9 & -1.0 \\
\hline Odisha & 34.1 & 31.0 & -3.1 \\
\hline Andhra Pradesh & 31.4 & 31.2 & -0.2 \\
\hline Rajasthan & 39.1 & 31.8 & -7.3 \\
\hline Lakshdweep & 26.8 & 32.0 & 5.2 \\
\hline Tripura & 24.3 & 32.3 & 8.0 \\
\hline Nagaland & 28.6 & 32.7 & 4.1 \\
\hline Telangana & 28.0 & 33.1 & 5.1 \\
\hline West Bengal & 32.5 & 33.8 & 1.3 \\
\hline Chhattisgarh & 37.6 & 34.6 & -3.0 \\
\hline Maharashtra & 34.4 & 35.2 & 0.8 \\
\hline Assam & 36.4 & 35.3 & -1.1 \\
\hline Karnataka & 36.2 & 35.4 & -0.8 \\
\hline Madhya Pradesh & 42.0 & 35.7 & -6.3 \\
\hline Gujarat & 38.5 & 39.0 & 0.5 \\
\hline Dadra \& Nagar Haveli and Daman \& Diu & 37.2 & 39.4 & 2.2 \\
\hline Jharkhand & 45.3 & 39.6 & -5.7 \\
\hline Uttar Pradesh & 46.3 & 39.7 & -6.6 \\
\hline Bihar & 48.3 & 42.9 & -5.4 \\
\hline Meghalaya & 43.8 & 46.5 & 2.7 \\
\hline
\end{tabular}

\begin{tabular}{|c|c|c|c|}
\hline (States/Uts)/Indicators) & $\begin{array}{l}\text { Children u } \\
\text { are waste } \\
\text { height)18 }\end{array}$ & $\begin{array}{l}\text { under } 5 \text { ye } \\
\text { ed (weigh } \\
\text { (\%) }\end{array}$ & $\begin{array}{l}\text { ears who } \\
\text { tt-for- }\end{array}$ \\
\hline ound & NFHS 4 1 & NFHS 5 & CHANGE \\
\hline VDIA & 21.0 & 19.3 & \begin{tabular}{|l|}
-1.7 \\
\end{tabular} \\
\hline handigarh & 10.9 & 8.4 & -2.5 \\
\hline Aizoram & 6.1 & 9.8 & 3.7 \\
\hline lanipur & 6.8 & 9.9 & 3.1 \\
\hline unjab & 15.6 & 10.6 & -5.0 \\
\hline ICT Delhi & 15.9 & 11.2 & -4.7 \\
\hline laryana & 21.2 & 11.5 & -9.7 \\
\hline leghalaya & 15.3 & 12.1 & -3.2 \\
\hline uducherry & 23.6 & 12.4 & -11.2 \\
\hline runachal Pradesh & 17.3 & 13.1 & -4.2 \\
\hline ttarakhand & 19.5 & 13.2 & -6.3 \\
\hline ikkim & 14.2 & 13.7 & -0.5 \\
\hline amil Nadu & 19.7 & 14.6 & -5.1 \\
\hline erala & 15.7 & 15.8 & 0.1 \\
\hline ndman \& Nicobar Islands & 18.9 & 16.0 & -2.9 \\
\hline ndhra Pradesh & 17.2 & 16.1 & -1.1 \\
\hline ajasthan & 23.0 & 16.8 & -6.2 \\
\hline ttar Pradesh & 17.9 & 17.3 & -0.6 \\
\hline imachal Pradesh & \begin{tabular}{c|c|}
13.7 \\
\end{tabular} & 17.4 & 3.7 \\
\hline akshdweep & 13.7 & 17.4 & 3.7 \\
\hline adakh & \begin{tabular}{r|r}
9.3 \\
\end{tabular} & 17.5 & 8.2 \\
\hline disha & 20.4 & 18.1 & -2.3 \\
\hline ipura & 16.8 & 18.2 & 1.4 \\
\hline hhattisgarh & 23.1 & 18.9 & -4.2 \\
\hline ammu \& Kashmir & 12.2 & 19.0 & 6.8 \\
\hline Iadhya Pradesh & 25.8 & 19.0 & -6.8 \\
\hline soa & 21.9 & 19.1 & -2.8 \\
\hline lagaland & 11.3 & 19.1 & 7.8 \\
\hline arnataka & 26.1 & 19.5 & -6.6 \\
\hline Vest Bengal & 20.3 & 20.3 & 0.0 \\
\hline adra \& Nagar Haveli and Daman \& Diu & 26.7 & 21.6 & -5.1 \\
\hline ssam & 17.0 & 21.7 & 4.7 \\
\hline elangana & 18.1 & 21.7 & 3.6 \\
\hline harkhand & 29.0 & 22.4 & -6.6 \\
\hline ihar & 20.8 & 22.9 & 2.1 \\
\hline ujarat & 26.4 & 25.1 & -1.3 \\
\hline laharashtra & 25.6 & 25.6 & 0.0 \\
\hline
\end{tabular}

\begin{tabular}{|c|c|c|c|}
\hline [StatestUts)]Indicators] & $\begin{array}{l}\text { Children u } \\
\text { are severe } \\
\text { (weight-fo }\end{array}$ & $\begin{array}{l}\text { Inder } 5 \text { yea } \\
\text { lly wasted } \\
\text { r-height)1 }\end{array}$ & $\begin{array}{l}\text { ars who } \\
\text { d } \\
19(\%)\end{array}$ \\
\hline Round & NFHS 4 & NFHS 5 & CHANGE \\
\hline INDIA & 7.5 & 7.7 & 0.2 \\
\hline Chandigarh & 3.9 & 2.3 & -1.6 \\
\hline Manipur & 2.2 & 3.4 & 1.2 \\
\hline Puducherry & 7.8 & 3.7 & -4.1 \\
\hline Punjab & 5.6 & 3.7 & -1.9 \\
\hline Dadra \& Nagar Haveli and Daman \& & 11.5 & 4.3 & -7.2 \\
\hline Haryana & 9.0 & 4.4 & -4.6 \\
\hline Meghalaya & 6.5 & 4.7 & -1.8 \\
\hline Uttarakhand & 9.0 & 4.7 & -4.3 \\
\hline Andman \& Nicobar Islands & 7.5 & 4.8 & -2.7 \\
\hline Mizoram & 2.3 & 4.9 & 2.6 \\
\hline NCT Delhi & 4.6 & 4.9 & 0.3 \\
\hline Tamil Nadu & 7.9 & 5.5 & -2.4 \\
\hline Kerala & 6.5 & 5.8 & -0.7 \\
\hline Andhra Pradesh & 4.5 & 6.0 & 1.5 \\
\hline Odisha & 6.4 & 6.1 & -0.3 \\
\hline Arunachal Pradesh & 8.0 & 6.5 & -1.5 \\
\hline Madhya Pradesh & 9.2 & 6.5 & -2.7 \\
\hline Sikkim & 5.9 & 6.6 & 0.7 \\
\hline Himachal Pradesh & 3.9 & 6.9 & 3.0 \\
\hline West Bengal & 6.5 & 7.1 & 0.6 \\
\hline Tripura & 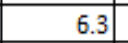 & 7.3 & 1.0 \\
\hline Uttar Pradesh & 6.0 & 7.3 & 1.3 \\
\hline Chhattisgarh & 8.4 & 7.5 & -0.9 \\
\hline Goa & 9.5 & 7.5 & -2.0 \\
\hline Rajasthan & 8.6 & 7.6 & -1.0 \\
\hline Nagaland & 4.2 & 7.9 & 3.7 \\
\hline Karnataka & 10.5 & 8.4 & -2.1 \\
\hline Telangana & 4.8 & 8.5 & 3.7 \\
\hline Lakshdweep & 2.9 & 8.7 & 5.8 \\
\hline Bihar & 7.0 & 8.8 & 1.8 \\
\hline Assam & 6.2 & 9.1 & 2.9 \\
\hline Jharkhand & 11.4 & 9.1 & -2.3 \\
\hline Ladakh & 5.1 & 9.1 & 4.0 \\
\hline Jammu \& Kashmir & 5.6 & 9.7 & 4.1 \\
\hline Gujarat & 9.5 & 10.6 & 1.1 \\
\hline Maharashtra & 9.4 & 10.9 & 1.5 \\
\hline
\end{tabular}




\section{Child Nutritional status}

Children under-five who are underweight

SDG target Achieved

SDG target Not Achieved

Improvement in the indicator

Decline in the indicator

No change

States arranged as per lowest to highest prevalence (NFHS-5)

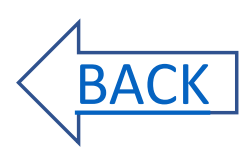

\begin{tabular}{|c|c|c|c|}
\hline [States'பts]|lndicators] & $\begin{array}{l}\text { Children } \\
\text { are under } \\
\text { age)18 (\% }\end{array}$ & $\begin{array}{l}\text { nder } 5 \text { ye } \\
\text { veight (w }\end{array}$ & $\begin{array}{l}\text { ears who } \\
\text { eight-for- }\end{array}$ \\
\hline Round & NFHS 4 & NFHS 5 & CHANGE \\
\hline INDIA & 35.8 & 32.1 & -3.7 \\
\hline Mizoram & 12.0 & 12.7 & 0.7 \\
\hline Sikkim & 14.2 & 13.1 & -1.1 \\
\hline Manipur & 13.8 & 13.3 & -0.5 \\
\hline Puducherry & 22.0 & 15.3 & -6.7 \\
\hline Arunachal Pradesh & 19.4 & 15.4 & -4.0 \\
\hline Punjab & 21.6 & 16.9 & -4.7 \\
\hline Kerala & 16.1 & 19.7 & 3.6 \\
\hline Ladakh & 18.7 & 20.4 & 1.7 \\
\hline Chandigarh & 24.5 & 20.6 & -3.9 \\
\hline Jammu \& Kashmir & 16.6 & 21.0 & 4.4 \\
\hline Uttarakhand & 26.6 & 21.0 & -5.6 \\
\hline Haryana & 29.4 & 21.5 & -7.9 \\
\hline NCT Delhi & 27.0 & 21.8 & -5.2 \\
\hline Tamil Nadu & 23.8 & 22.0 & -1.8 \\
\hline Andman \& Nicobar Islands & 21.6 & 23.7 & 2.1 \\
\hline Goa & 23.8 & 24.0 & 0.2 \\
\hline Himachal Pradesh & 21.2 & 25.5 & 4.3 \\
\hline Tripura & 24.1 & 25.6 & 1.5 \\
\hline Lakshdweep & 23.6 & 25.8 & 2.2 \\
\hline Meghalaya & 28.9 & 26.6 & -2.3 \\
\hline Nagaland & 16.7 & 26.9 & 10.2 \\
\hline Rajasthan & 36.7 & 27.6 & -9.1 \\
\hline Andhra Pradesh & 31.9 & 29.6 & -2.3 \\
\hline Odisha & 34.4 & 29.7 & -4.7 \\
\hline Chhattisgarh & 37.7 & 31.3 & -6.4 \\
\hline Telangana & 28.4 & 31.8 & 3.4 \\
\hline Uttar Pradesh & 39.5 & 32.1 & -7.4 \\
\hline West Bengal & 31.6 & 32.2 & 0.6 \\
\hline Assam & 29.8 & 32.8 & 3.0 \\
\hline Karnataka & 35.2 & 32.9 & -2.3 \\
\hline Madhya Pradesh & 42.8 & 33.0 & -9.8 \\
\hline Maharashtra & 36.0 & 36.1 & 0.1 \\
\hline Dadra \& Nagar Haveli and Daman \& Diu & 35.8 & 38.7 & 2.9 \\
\hline Jharkhand & 47.8 & 39.4 & -8.4 \\
\hline Gujarat & 39.3 & 39.7 & 0.4 \\
\hline Bihar & 43.9 & 41.0 & -2.9 \\
\hline
\end{tabular}

SDG 2030 target for children who are overweight: 1.9\%

\begin{tabular}{|c|c|c|c|}
\hline \multirow[t]{2}{*}{ [States'Uts])Indicators] } & \multicolumn{3}{|c|}{$\begin{array}{l}\text { Children under } 5 \text { years who } \\
\text { are overweight (weight-for- } \\
\text { height) } 20(\%)\end{array}$} \\
\hline & NFHS 4 & NFHS 5 & CHANGE \\
\hline INDIA & 2.1 & 3.4 & 1.3 \\
\hline Chandigarh & 1.1 & 1.9 & 0.8 \\
\hline Dadra \& Nagar Haveli and Daman \& Diu & 3.9 & 1.9 & -2.0 \\
\hline Madhya Pradesh & 1.7 & 2.0 & 0.3 \\
\hline Bihar & 1.2 & 2.4 & 1.2 \\
\hline Andhra Pradesh & 1.2 & 2.7 & 1.5 \\
\hline Goa & 3.7 & 2.8 & -0.9 \\
\hline Jharkhand & 1.5 & 2.8 & 1.3 \\
\hline Uttar Pradesh & 1.5 & 3.1 & 1.6 \\
\hline Karnataka & 2.6 & 3.2 & 0.6 \\
\hline Haryana & 3.1 & 3.3 & 0.2 \\
\hline Rajasthan & 2.1 & 3.3 & 1.2 \\
\hline Manipur & 3.1 & 3.4 & 0.3 \\
\hline Telangana & 0.7 & 3.4 & 2.7 \\
\hline Odisha & 2.6 & 3.5 & 0.9 \\
\hline Puducherry & 2.2 & 3.8 & 1.6 \\
\hline Gujarat & 1.9 & 3.9 & 2.0 \\
\hline Chhattisgarh & 2.9 & 4.0 & 1.1 \\
\hline Kerala & 3.4 & 4.0 & 0.6 \\
\hline Meghalaya & 3.9 & 4.0 & 0.1 \\
\hline NCT Delhi & 1.2 & 4.0 & 2.8 \\
\hline Maharashtra & 1.9 & 4.1 & 2.2 \\
\hline Punjab & 2.3 & 4.1 & 1.8 \\
\hline Uttarakhand & 3.5 & 4.1 & 0.6 \\
\hline Tamil Nadu & 5.0 & 4.3 & -0.7 \\
\hline West Bengal & 2.1 & 4.3 & 2.2 \\
\hline Assam & 2.3 & 4.9 & 2.6 \\
\hline Nagaland & 3.8 & 4.9 & 1.1 \\
\hline Andman \& Nicobar Islands & 3.0 & 5.4 & 2.4 \\
\hline Himachal Pradesh & 1.9 & 5.7 & 3.8 \\
\hline Tripura & 3.0 & 8.2 & 5.2 \\
\hline Jammu \& Kashmir & 5.7 & 9.6 & 3.9 \\
\hline Sikkim & 8.6 & 9.6 & 1.0 \\
\hline Arunachal Pradesh & 4.9 & 9.7 & 4.8 \\
\hline Mizoram & 4.2 & 10.0 & 5.8 \\
\hline Lakshdweep & 1.6 & 10.5 & 8.9 \\
\hline Ladakh & 4.0 & 13.4 & 9.4 \\
\hline
\end{tabular}




\section{Nutritional Status of Adults}

\section{Women underweight}

\begin{tabular}{|c|c|c|c|}
\hline [States'UTs] Indicators & $\begin{array}{l}\text { Women w } \\
\text { Index (BM } \\
\text { normal (B } \\
(\%)\end{array}$ & $\begin{array}{l}\text { hose Boo } \\
\text { ) is belo } \\
\text { MI }<18.5 \text { K }\end{array}$ & $\begin{array}{l}\text { dy Mass } \\
\text { wg } \\
\mathrm{kg} / \mathrm{m} 2) 21\end{array}$ \\
\hline Round & NFHS 4 & NFHS 5 & CHANGE \\
\hline INDIA & 22.9 & 18.7 & -4.2 \\
\hline Ladakh & 10.5 & \begin{tabular}{r|r}
4.4 \\
\end{tabular} & -6.1 \\
\hline Jammu \& Kashmir & 12.2 & 5.2 & -7.0 \\
\hline Mizoram & 8.4 & 5.3 & -3.1 \\
\hline Arunachal Pradesh & 8.5 & 5.7 & -2.8 \\
\hline Sikkim & 6.4 & 5.8 & -0.6 \\
\hline Manipur & 8.8 & 7.2 & -1.6 \\
\hline Lakshdweep & 13.5 & 8.0 & -5.5 \\
\hline Puducherry & 11.3 & 9.0 & -2.3 \\
\hline Andman \& Nicobar Islands & 13.1 & 9.4 & -3.7 \\
\hline NCT Delhi & 14.9 & 10.0 & -4.9 \\
\hline Kerala & 9.7 & 10.1 & 0.4 \\
\hline Meghalaya & 12.1 & 10.8 & -1.3 \\
\hline Nagaland & 12.3 & 11.1 & -1.2 \\
\hline Tamil Nadu & 14.6 & 12.6 & -2.0 \\
\hline Punjab & 11.7 & 12.7 & 1.0 \\
\hline Chandigarh & 13.3 & 13.0 & -0.3 \\
\hline Goa & 14.7 & 13.8 & -0.9 \\
\hline Himachal Pradesh & 16.2 & 13.9 & -2.3 \\
\hline Uttarakhand & 18.4 & 13.9 & -4.5 \\
\hline Andhra Pradesh & 17.6 & 14.8 & -2.8 \\
\hline West Bengal & 21.3 & 14.8 & -6.5 \\
\hline \begin{tabular}{|l} 
Haryana \\
\end{tabular} & 15.8 & 15.1 & -0.7 \\
\hline Tripura & 18.9 & 16.2 & -2.7 \\
\hline Karnataka & 20.7 & 17.2 & -3.5 \\
\hline Assam & 25.7 & 17.6 & -8.1 \\
\hline Telangana & 22.9 & 18.8 & -4.1 \\
\hline Uttar Pradesh & 25.3 & 19.0 & -6.3 \\
\hline Rajasthan & 27.0 & 19.6 & -7.4 \\
\hline Maharashtra & 23.5 & 20.8 & -2.7 \\
\hline Odisha & 26.5 & 20.8 & -5.7 \\
\hline Madhya Pradesh & 28.4 & 23.0 & -5.4 \\
\hline Chhattisgarh & 26.7 & 23.1 & -3.6 \\
\hline Dadra \& Nagar Haveli and Daman \& Diu & 23.4 & 25.1 & 1.7 \\
\hline Gujarat & 27.2 & 25.2 & -2.0 \\
\hline Bihar & 30.4 & 25.6 & -4.8 \\
\hline Jharkhand & 31.5 & 26.2 & -5.3 \\
\hline
\end{tabular}

Men underweight

\begin{tabular}{|c|c|c|c|}
\hline \multirow[t]{2}{*}{ [States'பTs])!ndicators } & \multicolumn{3}{|c|}{$\begin{array}{l}\text { Men whose Body Mass Index } \\
(\mathrm{BMI}) \text { is below normal (BMI } \\
<18.5 \mathrm{~kg} / \mathrm{m} 2)(\%)\end{array}$} \\
\hline & \begin{tabular}{|l|} 
NFHS 4 \\
\end{tabular} & NFHS 5 & Change \\
\hline INDIA & 20.2 & 16.2 & -4.0 \\
\hline Ladakh & 11.2 & 2.1 & -9.1 \\
\hline Andman \& Nicobar Islands & 8.7 & 4.0 & -4.7 \\
\hline Jammu \& Kashmir & 11.5 & 4.3 & -7.2 \\
\hline Arunachal Pradesh & 8.3 & 4.9 & -3.4 \\
\hline Sikkim & 2.4 & 4.9 & 2.5 \\
\hline Mizoram & 7.3 & 5.1 & -2.2 \\
\hline Lakshdweep & 8.2 & 5.5 & -2.7 \\
\hline Nagaland & 11.5 & 7.5 & -4.0 \\
\hline Manipur & 11.1 & 8.0 & -3.1 \\
\hline Meghalaya & 11.6 & 9.0 & -2.6 \\
\hline NCT Delhi & 17.7 & 9.1 & -8.6 \\
\hline Kerala & 8.5 & 10.0 & 1.5 \\
\hline Puducherry & 10.2 & 11.1 & 0.9 \\
\hline Himachal Pradesh & 18.0 & 11.8 & -6.2 \\
\hline Tamil Nadu & 12.4 & 12.1 & -0.3 \\
\hline Tripura & 15.7 & 12.4 & -3.3 \\
\hline Goa & 10.8 & 12.5 & 1.7 \\
\hline Punjab & 10.9 & 12.5 & 1.6 \\
\hline Assam & 20.7 & 13.4 & -7.3 \\
\hline Rajasthan & 22.7 & 14.0 & -8.7 \\
\hline Karnataka & 16.5 & 14.3 & -2.2 \\
\hline Haryana & 11.3 & 14.5 & 3.2 \\
\hline Chandigarh & 21.7 & 15.1 & -6.6 \\
\hline West Bengal & 19.9 & 15.1 & -4.8 \\
\hline Odisha & 19.5 & 15.3 & -4.2 \\
\hline Maharashtra & 19.1 & 16.2 & -2.9 \\
\hline Telangana & 21.5 & 16.2 & -5.3 \\
\hline Uttarakhand & 16.1 & 16.2 & 0.1 \\
\hline Andhra Pradesh & 14.8 & 16.5 & 1.7 \\
\hline Jharkhand & 23.8 & 17.1 & -6.7 \\
\hline Chhattisgarh & 24.1 & 17.4 & -6.7 \\
\hline Uttar Pradesh & 25.9 & 17.9 & -8.0 \\
\hline Dadra \& Nagar Haveli and Daman \& Diu & 16.3 & 18.3 & 2.0 \\
\hline Madhya Pradesh & 28.4 & 20.8 & -7.6 \\
\hline Gujarat & 24.7 & 20.9 & -3.8 \\
\hline Bihar & 25.4 & 21.5 & -3.9 \\
\hline
\end{tabular}

States arranged as per lowest to highest prevalence (NFHS-5)

$$
\langle\overrightarrow{B A C K} \underset{\text { NEXT }}{\longrightarrow}
$$


Nutritional Status of Adults

Improvement in the indicator

Decline in the indicator

No change

States arranged as per lowest to highest prevalence (NFHS-5)

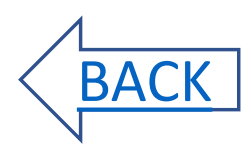

Women overweight

\begin{tabular}{|c|c|c|c|}
\hline [States'பTs]"lndicators & $\begin{array}{l}\text { Women w } \\
\text { or obese ( } \\
\mathrm{kg} / \mathrm{m} 2) 21\end{array}$ & $\begin{array}{l}10 \text { are ove } \\
3 \mathrm{MI} \geq 25.0 \\
\% \text { ) }\end{array}$ & erweight \\
\hline Round & NFHS 4 & NFHS 5 & Change \\
\hline INDIA & 20.6 & 24.0 & 3.4 \\
\hline Meghalaya & 12.2 & 11.5 & -0.7 \\
\hline Jharkhand & 10.3 & 11.9 & 1.6 \\
\hline Rajasthan & 14.1 & 12.9 & -1.2 \\
\hline Chhattisgarh & 11.9 & 14.1 & 2.2 \\
\hline Nagaland & 16.2 & 14.4 & -1.8 \\
\hline Assam & 13.2 & 15.2 & 2.0 \\
\hline Bihar & 11.7 & 15.9 & 4.2 \\
\hline Madhya Pradesh & 13.6 & 16.6 & 3.0 \\
\hline Uttar Pradesh & 16.5 & 21.3 & 4.8 \\
\hline Tripura & 16.0 & 21.5 & 5.5 \\
\hline Gujarat & 23.7 & 22.6 & -1.1 \\
\hline West Bengal & 19.9 & 22.7 & 2.8 \\
\hline Odisha & 16.5 & 23.0 & 6.5 \\
\hline Maharashtra & 23.4 & 23.4 & 0.0 \\
\hline Arunachal Pradesh & 18.8 & 23.9 & 5.1 \\
\hline Mizoram & 21.0 & 24.2 & 3.2 \\
\hline Dadra \& Nagar Haveli and Daman \& Diu & 23.3 & 26.8 & 3.5 \\
\hline Ladakh & 16.3 & 28.3 & 12.0 \\
\hline Jammu \& Kashmir & 29.3 & 29.3 & 0.0 \\
\hline Uttarakhand & 20.4 & 29.7 & 9.3 \\
\hline Karnataka & 23.3 & 30.1 & 6.8 \\
\hline Telangana & 28.6 & 30.1 & 1.5 \\
\hline Himachal Pradesh & 28.6 & 30.4 & 1.8 \\
\hline Haryana & 21.0 & 33.1 & 12.1 \\
\hline Lakshdweep & 40.6 & 33.5 & -7.1 \\
\hline Manipur & 26.0 & 34.1 & 8.1 \\
\hline Sikkim & 26.7 & 34.7 & 8.0 \\
\hline Goa & 33.5 & 36.1 & 2.6 \\
\hline Andhra Pradesh & 33.2 & 36.3 & 3.1 \\
\hline Andman \& Nicobar Islands & 31.8 & 38.1 & 6.3 \\
\hline Kerala & 32.4 & 38.1 & 5.7 \\
\hline Tamil Nadu & 30.9 & 40.4 & 9.5 \\
\hline Punjab & 31.3 & 40.8 & 9.5 \\
\hline NCT Delhi & 33.5 & 41.3 & 7.8 \\
\hline Chandigarh & 41.5 & 44.0 & 2.5 \\
\hline Puducherry & 36.7 & 46.2 & 9.5 \\
\hline
\end{tabular}

\section{Men overweight}

\begin{tabular}{|c|c|c|c|}
\hline [States'UTs]) Indicators & $\begin{array}{l}\text { Men who } \\
\text { obese (BI }\end{array}$ & $\begin{array}{l}\text { are overwe } \\
\mathrm{MI} \geq 25.0 \mathrm{~kg}\end{array}$ & $\begin{array}{l}\text { eight or } \\
/ / \mathrm{m} 2)(\%)\end{array}$ \\
\hline Round & NFHS4 & NFHS5 & CHANGE \\
\hline INDIA & 18.9 & 22.9 & 4.0 \\
\hline Meghalaya & 10.1 & 13.9 & 3.8 \\
\hline Bihar & 12.6 & 14.7 & 2.1 \\
\hline Chhattisgarh & 10.2 & 14.9 & 4.7 \\
\hline Rajasthan & 13.2 & 15.0 & 1.8 \\
\hline Jharkhand & 11.1 & 15.1 & 4.0 \\
\hline Madhya Pradesh & 10.9 & 15.6 & 4.7 \\
\hline Assam & 12.9 & 16.2 & 3.3 \\
\hline West Bengal & 14.2 & 16.2 & 2.0 \\
\hline Uttar Pradesh & 12.5 & 18.5 & 6.0 \\
\hline Gujarat & 19.7 & 19.9 & 0.2 \\
\hline Dadra \& Nagar Haveli and Daman \& Diu & 26.3 & 21.4 & -4.9 \\
\hline Odisha & 17.2 & 22.2 & 5.0 \\
\hline Tripura & 15.9 & 23.4 & 7.5 \\
\hline Nagaland & 13.9 & 23.9 & 10.0 \\
\hline Maharashtra & 23.8 & 24.7 & 0.9 \\
\hline Uttarakhand & 17.7 & 27.1 & 9.4 \\
\hline Arunachal Pradesh & 20.6 & 27.6 & 7.0 \\
\hline Haryana & 20.0 & 28.3 & 8.3 \\
\hline Manipur & 19.8 & 30.3 & 10.5 \\
\hline Himachal Pradesh & 22.0 & 30.6 & 8.6 \\
\hline Karnataka & 22.1 & 30.9 & 8.8 \\
\hline Andhra Pradesh & 33.5 & 31.1 & -2.4 \\
\hline Jammu \& Kashmir & 20.5 & 31.6 & 11.1 \\
\hline Mizoram & 20.9 & 31.9 & 11.0 \\
\hline Punjab & 27.8 & 32.2 & 4.4 \\
\hline Telangana & 24.2 & 32.3 & 8.1 \\
\hline Goa & 32.6 & 32.6 & 0.0 \\
\hline Chandigarh & 32.0 & 34.4 & 2.4 \\
\hline Sikkim & 34.8 & 36.3 & 1.5 \\
\hline Kerala & 28.5 & 36.4 & 7.9 \\
\hline Tamil Nadu & 28.2 & 37.0 & 8.8 \\
\hline Ladakh & 18.8 & 37.8 & 19.0 \\
\hline NCT Delhi & 24.6 & 38.0 & 13.4 \\
\hline Lakshdweep & 24.1 & 41.3 & 17.2 \\
\hline Puducherry & 37.1 & 43.3 & 6.2 \\
\hline Andman \& Nicobar Islands & 38.2 & 45.3 & 7.1 \\
\hline
\end{tabular}




\section{Anemia in Children and adolescents}

Anemia in children (6-59)months

\begin{tabular}{|c|c|c|c|}
\hline \multirow[t]{2}{*}{ [States'UTs] Indicators } & \multicolumn{3}{|c|}{$\begin{array}{l}\text { Children age } 6-59 \text { months } \\
\text { who are anaemic }(<11.0 \text { g'd }) 22 \\
(\%)\end{array}$} \\
\hline & NFHS4 & NFHS5 & CHANGE \\
\hline INDIA & 58.6 & 67.1 & 8.5 \\
\hline Kerala & 35.7 & 39.4 & 3.7 \\
\hline Andman \& Nicobar Islands & 49.0 & 40.0 & -9.0 \\
\hline Nagaland & 26.4 & 42.7 & 16.3 \\
\hline Manipur & 23.9 & 42.8 & 18.9 \\
\hline Lakshdweep & 53.6 & 43.1 & -10.5 \\
\hline Meghalaya & 48.0 & 45.1 & -2.9 \\
\hline Mizoram & 19.3 & 46.4 & 27.1 \\
\hline Goa & 48.3 & 53.2 & 4.9 \\
\hline Chandigarh & 73.1 & 54.6 & -18.5 \\
\hline Himachal Pradesh & 53.7 & 55.4 & 1.7 \\
\hline Sikkim & 55.1 & 56.4 & 1.3 \\
\hline Arunachal Pradesh & 54.2 & 56.6 & 2.4 \\
\hline Tamil Nadu & 50.7 & 57.4 & 6.7 \\
\hline Uttarakhand & 59.8 & 58.8 & -1.0 \\
\hline Andhra Pradesh & 58.6 & 63.2 & 4.6 \\
\hline Puducherry & 44.9 & 64.0 & 19.1 \\
\hline Odisha & 44.6 & 64.2 & 19.6 \\
\hline Tripura & 48.3 & 64.3 & 16.0 \\
\hline Karnataka & 60.9 & 65.5 & 4.6 \\
\hline Lttar Pradesh & 63.2 & 66.4 & 3.2 \\
\hline Chhattisgarh & 41.6 & 67.2 & 25.6 \\
\hline Jharkhand & 69.9 & 67.5 & -2.4 \\
\hline Assam & 35.7 & 68.4 & 32.7 \\
\hline Maharashtra & 53.8 & 68.9 & 15. \\
\hline West Bengal & 54.2 & 69.0 & 14.8 \\
\hline NCT Delhi & 59.7 & 69.2 & 9.5 \\
\hline Bihar & 63.5 & 69.4 & 5.9 \\
\hline Telangana & 60.7 & 70.0 & 9.3 \\
\hline Haryana & 71.7 & 70.4 & -1.3 \\
\hline Puniab & 56.6 & 71.1 & 14.5 \\
\hline Rajasthan & 60.3 & 71.5 & 11.2 \\
\hline Jammu \& Kashmir & 53.8 & 72.7 & 18.9 \\
\hline Madhya Pradesh & 68.9 & 72.7 & 3.8 \\
\hline Dadra \& Nagar Haveli and Daman \& Diu & 82.0 & 75.8 & -6.2 \\
\hline Gujarat & 62.6 & 79.7 & 17.1 \\
\hline Ladakh & 91.4 & 92.5 & 1. \\
\hline
\end{tabular}

Adolescent girls (15-19 years)

\begin{tabular}{|c|c|c|c|}
\hline (StatestUTs)|lndicators & $\begin{array}{l}\text { All women } \\
\text { who are ar }\end{array}$ & age $15-19 y$ & \\
\hline Round & NFHS 4 & NFHS5 5 & hange \\
\hline INDIA & 54.1 & 59.1 & 5.1 \\
\hline Manipur & 21.1 & 27.9 & 6.8 \\
\hline Lakshdweep & 59.0 & 31.4 & -27.6 \\
\hline Kerala & 37.8 & 32.5 & -5.3 \\
\hline Nagaland & 26.3 & 33.9 & 7.6 \\
\hline Mizoram & 21.3 & 34.9 & 13.6 \\
\hline Uttarakhand & 46.4 & 40.9 & -5.5 \\
\hline Goa & 30.5 & 44.5 & 14.0 \\
\hline Andman \& Nicobar Islands & 68.1 & 44.9 & -23.2 \\
\hline Sikkim & 48.7 & \begin{tabular}{c|c}
46.7 \\
\end{tabular} & -2.0 \\
\hline Arunachal Pradesh & 48.2 & 48.5 & 0.3 \\
\hline Karnataka & 45.3 & 49.4 & 4. \\
\hline NCT Delhi & 55.1 & 51.6 & -3.5 \\
\hline Meghalaya & 52.1 & 52.5 & 0.4 \\
\hline Tamil Nadu & 54.2 & 52.9 & -1.3 \\
\hline Uttar Pradesh & \begin{tabular}{c|c}
53.7 \\
\end{tabular} & 52.9 & -0.8 \\
\hline Himachal Pradesh & 52.7 & 53.2 & 0.5 \\
\hline Maharashtra & 49.7 & 57.2 & 7.5 \\
\hline Chandigarh & 74.7 & 57.7 & -17.0 \\
\hline Madhya Pradesh & 53.2 & 58.1 & 4.5 \\
\hline Puducherry & 55.0 & 58.4 & 3.4 \\
\hline Rajasthan & 49.1 & 59.4 & 10.3 \\
\hline Andhra Pradesh & 61.1 & 60.1 & -1.0 \\
\hline Puniab & 58.0 & 60.3 & 2.3 \\
\hline Chhattisgarh & 45.5 & 61.4 & 15.9 \\
\hline Haryana & 62.7 & 62.3 & -0.4 \\
\hline Dadra \& Nagar Haveli and Daman \& Diu & 75.9 & 63.9 & -12.0 \\
\hline $\begin{array}{l}\text { Telangana } \\
\end{array}$ & 59.7 & 64.7 & 5.0 \\
\hline Odisha & 51.0 & 65.5 & 14.5 \\
\hline Bihar & 61.0 & 65.7 & 4.7 \\
\hline Jharkhand & 65.0 & 65.8 & 0.8 \\
\hline Assam & 42.7 & 67.0 & 24.3 \\
\hline Tripura & 52.2 & 67.9 & 15.7 \\
\hline Gujarat & 56.5 & 69.0 & 12.5 \\
\hline West Bengal & 62.2 & 70.8 & 8.6 \\
\hline Jammu \& Kashmir & 49.9 & 76.2 & 26.3 \\
\hline Ladakh & 81.6 & 96.9 & 15.3 \\
\hline
\end{tabular}

Adolescent boys (15-19 years)

\begin{tabular}{|c|c|c|c|}
\hline \multirow[t]{2}{*}{ (States:UTs).|lndicators } & \multicolumn{3}{|c|}{$\begin{array}{l}\text { Men age 15-19 years who are } \\
\text { anaemic }(<13.0 \text { gdd } 22(\%)\end{array}$} \\
\hline & NFHS 4 & NFHS5 & Change \\
\hline INDIA & 29.2 & 31.1 & 1.9 \\
\hline Manipur & 9.2 & 7.8 & -1.4 \\
\hline Goa & 6.6 & 15.8 & 9.2 \\
\hline Sikkim & 16.7 & 17.6 & 0.9 \\
\hline Andhra Pradesh & 29.3 & 18.7 & -10.6 \\
\hline NCT Delhi & 25.9 & 18.9 & -7.0 \\
\hline Nagaland & 12.2 & 19.6 & 7.4 \\
\hline Mizoram & 14.4 & 21.5 & 7.1 \\
\hline Himachal Pradesh & 25.0 & 22.1 & -2.9 \\
\hline Tamil Nadu & 26.0 & 24.6 & -1.4 \\
\hline Arunachal Pradesh & 22.9 & 24.9 & 2.0 \\
\hline Telangana & 19.2 & 25.1 & 5.9 \\
\hline Karnataka & 24.5 & 26.5 & 2.0 \\
\hline Andman \& Nicobar Islands & 43.0 & 27.1 & -15.9 \\
\hline Tripura & 22.0 & 27.2 & 5.2 \\
\hline Kerala & 14.3 & 27.4 & 13.1 \\
\hline Uttarakhand & 22.2 & 27.6 & 5.4 \\
\hline Maharashtra & 27.5 & 27.9 & 0.4 \\
\hline Uttar Pradesh & 31.5 & 28.2 & -3.3 \\
\hline Haryana & 29.7 & 29.9 & 0.2 \\
\hline Odisha & 30.3 & 30.0 & -0.3 \\
\hline Meghalaya & 25.2 & 30.1 & 4.9 \\
\hline Madhya Pradesh & 36.5 & 30.5 & -6.0 \\
\hline Puducherry & 40.6 & 30.7 & -9.9 \\
\hline Chhattisgarh & 27.4 & 31.5 & 4.1 \\
\hline Puniab & 30.8 & 32.7 & 1.9 \\
\hline Rajasthan & 22.1 & 34.0 & 11.9 \\
\hline Bihar & 37.8 & 34.8 & -3.0 \\
\hline Gujarat & 31.9 & 36.0 & 4.11 \\
\hline Dadra \& Nagar Haveli and Daman \& Diu & 36.1 & 37.0 & 0.9 \\
\hline West Bengal & 31.7 & 38.7 & 7.0 \\
\hline Assam & 23.5 & 39.6 & 16.1 \\
\hline Jharkhand & 35.3 & 39.7 & 4.4 \\
\hline Jammu \& Kashmir & 29.5 & 53.5 & 24.0 \\
\hline Ladakh & 57.6 & 93.1 & 35.5 \\
\hline Chandigarh & 22.4 & na & na \\
\hline Lakshdweep & $\mathrm{na}$ & na & $\mathrm{na}$ \\
\hline
\end{tabular}

States arranged as per lowest to highest prevalence (NFHS-5)

Anemia in boys:

Data for Ladakh (NFHS 5 ,

based on 25-49 unweighted

cases

$\angle \mathrm{BACK}$ in the indicator 


\section{Anemia in Adults}

Anemia in pregnant women $15-49$ years

\begin{tabular}{|c|c|c|c|}
\hline [StatedUtsindicators] & $\begin{array}{r}\text { Pregnant } \\
\text { yea } \\
\text { anae }\end{array}$ & $\begin{array}{l}\text { Women ad } \\
\text { is who ar } \\
\text { mic_NFH }\end{array}$ & $\begin{array}{l}\text { age } 15-49 \\
\text { re } \\
455\end{array}$ \\
\hline Found & NFHS 4 & NFHS 5 & Change \\
\hline INDIA & 50.4 & 52.2 & 1.8 \\
\hline Lakshadweep & 39.0 & 20.9 & -18.1 \\
\hline Nagaland & 32.7 & 22.2 & -10.5 \\
\hline Arrunachal Pradesh & 37.8 & 27.9 & -9.9 \\
\hline Kerala & 22.6 & 31.4 & 8.8 \\
\hline Manipur & 26.0 & 32.4 & 6.4 \\
\hline Mizoram & 27.0 & 34.0 & 7.0 \\
\hline Sikkim & 23.6 & 40.7 & 17.1 \\
\hline Goa & 26.7 & 41.0 & 14.3 \\
\hline Himachal pradesh & 50.4 & \begin{tabular}{l|l}
42.2 \\
\end{tabular} & -8.2 \\
\hline NCT Delhi & 46.1 & 42.2 & -3.9 \\
\hline Puducherry & 26.0 & 42.5 & 16.5 \\
\hline Jammu and Kashmir & 46.9 & 44.1 & -2.8 \\
\hline Meghalaya & 53.3 & 45.0 & -8.3 \\
\hline Karnataka & 45.4 & 45.7 & 0.3 \\
\hline Maharastra & 49.3 & \begin{tabular}{l|l|}
45.7 \\
\end{tabular} & -3.6 \\
\hline Uttar Pradesh & 51.0 & 45.9 & -5.1 \\
\hline Rajasthan & 46.6 & \begin{tabular}{l|l}
46.3 \\
\end{tabular} & -0.3 \\
\hline Uttarakhand & \begin{tabular}{l|l}
46.5 \\
\end{tabular} & 46.4 & -0.1 \\
\hline Tamil Nadu & 44.4 & 48.3 & 3.9 \\
\hline Punjab & 42.0 & 51.7 & 9.7 \\
\hline Chattisgarh & 41.5 & 51.8 & 10.3 \\
\hline Madhya Pradesh & 54.6 & 52.9 & -1.7 \\
\hline Telangana & \begin{tabular}{l|l}
48.2 \\
\end{tabular} & 53.2 & 5.0 \\
\hline Andaman \& Nicobar & 61.4 & 53.7 & -7.7 \\
\hline Andhra pradesh & 52.9 & 53.7 & 0.8 \\
\hline Assam & 44.8 & 54.2 & 9.4 \\
\hline Haryana & 55.0 & 56.5 & 1.5 \\
\hline Jharkhand & 62.6 & 56.8 & -5.8 \\
\hline Dadar\& nagar haveli and Daman \& Diu & 62.3 & 60.7 & -1.6 \\
\hline Tripura & 54.4 & 61.5 & 7.1 \\
\hline Odisha & 47.6 & 61.8 & 14.2 \\
\hline West bengal & 53.6 & 62.3 & 8.7 \\
\hline Gujarat & 51.3 & 62.6 & 11.3 \\
\hline BHAR & 58.3 & 63.1 & 4.8 \\
\hline Ladakh & 79.3 & 78.1 & -1.2 \\
\hline Chandigarh & $\mathrm{na}$ & $\mathrm{na}$ & $\mathrm{na}$ \\
\hline
\end{tabular}

SDG 2030 target for Anemia among women (1549 years) : $25.6 \%$

\begin{tabular}{|c|c|c|c|}
\hline [StatedUtsinndicators] & $\begin{array}{l}\text { All wome } \\
\text { who are }\end{array}$ & $\begin{array}{c}n \text { age } 15-4 \\
\text { naemic_l }\end{array}$ & $\begin{array}{l}-49 \text { years } \\
\text { NFHS } 5\end{array}$ \\
\hline Round & NFHS 4 & NFHS 5 & Change \\
\hline INDIA & 53.1 & 57.0 & 3.9 \\
\hline Lakshadweep & 46.0 & 25.8 & -20.2 \\
\hline Nagaland & 27.9 & 28.9 & 1.0 \\
\hline Manipur & 26.4 & 29.4 & 3.0 \\
\hline Mizoram & 24.8 & 34.8 & 10.0 \\
\hline Kerala & 34.3 & 36.3 & 2.0 \\
\hline Goa & 31.3 & 39.0 & 7.7 \\
\hline Arunachal Pradesh & 43.2 & 40.3 & -2.9 \\
\hline Sikkim & 34.9 & 42.1 & 7.2 \\
\hline Uttarakhand & 45.2 & \begin{tabular}{l|l}
42.6 \\
\end{tabular} & -2.6 \\
\hline Karnataka & 44.8 & 47.8 & 3.0 \\
\hline NCT Delhi & 54.3 & 49.9 & -4.4 \\
\hline Uttar Pradesh & 52.4 & 50.4 & -2.0 \\
\hline Himachal pradesh & 53.5 & 53.0 & -0.5 \\
\hline Tamil Nadu & 55.0 & 53.4 & -1.6 \\
\hline Meghalaya & 56.2 & 53.8 & -2.4 \\
\hline Maharastra & 48.0 & 54.2 & 6.2 \\
\hline Rajasthan & 46.8 & 54.4 & 7.6 \\
\hline Madhya Pradesh & 52.5 & 54.7 & 2.2 \\
\hline Puducherry & 52.4 & 55.1 & 2.7 \\
\hline Andaman \& Nicobar & 65.7 & 57.5 & -8.2 \\
\hline Telangana & 56.6 & 57.6 & 1.0 \\
\hline Puniab & 53.5 & 58.7 & 5.2 \\
\hline Andhra pradesh & 60.0 & 58.8 & -1.2 \\
\hline Chandigarh & 75.9 & 60.3 & -15.6 \\
\hline Haryana & 62.7 & 60.4 & -2.3 \\
\hline Chattisgarh & 47.0 & 60.8 & 13.8 \\
\hline Dadar\& nagar haveli and Daman \& Diu & 72.9 & 62.5 & -10.4 \\
\hline BHAR & 60.3 & 63.5 & 3.2 \\
\hline Odisha & 51.0 & 64.3 & 13.3 \\
\hline Gujarat & 54.9 & 65.0 & 10.1 \\
\hline Jharkhand & 65.2 & 65.3 & 0.1 \\
\hline Assam & 46.0 & 65.9 & 19.9 \\
\hline Jammu and Kashmir & 48.9 & 65.9 & 17.0 \\
\hline Tripura & 54.5 & 67.2 & 12.7 \\
\hline West bengal & 62.5 & 71.4 & 8.9 \\
\hline Ladakh & 78.4 & 92.8 & 14.4 \\
\hline
\end{tabular}

\section{Anemia in men $15-49$ years}

\begin{tabular}{|c|c|c|c|}
\hline \multirow{2}{*}{$\begin{array}{l}\text { [StatedUtsilndicators] } \\
\text { Round } \\
\end{array}$} & \multicolumn{3}{|c|}{$\begin{array}{c}\text { Men age } 15-49 \text { years who are } \\
\text { anaemic_NFHS } 5\end{array}$} \\
\hline & NFHS 4 & NFHS 5 & Change \\
\hline INDIA & 22.7 & 25.0 & 2.3 \\
\hline Lakshadweep & 11.4 & 5.6 & -5.8 \\
\hline Manipur & 9.5 & 6.0 & -3.5 \\
\hline Chandigarh & 19.3 & 8.1 & -11.2 \\
\hline Nagaland & 11.7 & 10.0 & -1.7 \\
\hline Goa & 11.0 & 12.0 & 1.0 \\
\hline NCT Delhi & 21.7 & 12.6 & -9.1 \\
\hline Uttarakhand & 15.6 & 15.1 & -0.5 \\
\hline Tamil Nadu & 20.4 & 15.2 & -5.2 \\
\hline Telangana & 15.3 & 15.3 & 0.0 \\
\hline Mizoram & 12.1 & 15.6 & 3.5 \\
\hline Andaman \& Nicobar & 30.8 & 16.1 & -14.7 \\
\hline Andhra pradesh & 27.0 & 16.2 & -10.8 \\
\hline Kerala & 11.8 & 17.8 & 6.0 \\
\hline Himachal pradesh & 20.1 & 18.6 & -1.5 \\
\hline Sikkim & 15.8 & 18.7 & 2.9 \\
\hline Haryana & 20.9 & 18.9 & -2.0 \\
\hline Puducherry & 15.9 & 19.5 & 3.6 \\
\hline Karnataka & 18.3 & 19.6 & 1.3 \\
\hline Arunachal Pradesh & 18.7 & 21.4 & 2.7 \\
\hline Uttar Pradesh & 23.7 & 21.5 & -2.2 \\
\hline Maharastra & 17.7 & 21.9 & 4.2 \\
\hline Madhya Pradesh & 25.5 & 22.4 & -3.1 \\
\hline Punjab & 25.9 & 22.6 & -3.3 \\
\hline Rajasthan & 17.2 & 23.2 & 6.0 \\
\hline Dadar\& nagar haveli and Daman \& Diu & 27.6 & 24.6 & -3.0 \\
\hline Meghalaya & 32.4 & 25.5 & -6.9 \\
\hline Gujarat & 21.6 & 26.6 & 5.0 \\
\hline Chattisgarh & 22.1 & 27.0 & 4.9 \\
\hline Ddisha & 28.3 & 28.5 & 0.2 \\
\hline BHAR & 32.3 & 29.5 & -2.8 \\
\hline Jharkhand & 29.8 & 29.6 & -0.2 \\
\hline Assam & 25.4 & 36.0 & 10.6 \\
\hline Jammu and Kashmir & 20.4 & 36.7 & 16.3 \\
\hline Tripura & 24.7 & 36.9 & 12.2 \\
\hline West bengal & 30.3 & 38.9 & 8.6 \\
\hline Ladakh & 41.2 & 75.6 & 34.4 \\
\hline
\end{tabular}

States arranged as per lowest to highest prevalence (NFHS-5)

Anemia in pregnant women Data for Lakshadweep (in NFHS 5) based on 25-49 unweighted cases

Anemia in men $15-49$ years:

Data for Ladakh (in NFHS 5) and Chandigarh (in NFHS 4) is based on 25-49 unweighted cases $\angle \mathrm{BACK}$ 


\section{Consumption of supplements}

Mothers consumed IFA for 100 days

\begin{tabular}{|l|r|r|r||}
\hline \multicolumn{1}{|c|}{ (States/UTs)/Indicators } & \multicolumn{3}{|c|}{$\begin{array}{l}\text { Mothers who consumed iron } \\
\text { folic acid for } 100 \text { days or }\end{array}$} \\
\hline Round & NFHS 4 & NFHS 5 & Change \\
\hline INDIA & 30.3 & 44.1 & 13.8 \\
\hline Goa & 67.4 & 87.5 & 20.1 \\
\hline Puducherry & 66.3 & 84.1 & 17.8 \\
\hline Tamil Nadu & 64.0 & 82.5 & 18.5 \\
\hline Andman \& Nicobar Islands & 58.4 & 80.9 & 22.5 \\
\hline Lakshdweep & 81.7 & 80.1 & -1.6 \\
\hline Kerala & 67.1 & 80.0 & 12.9 \\
\hline Chandigarh & 44.9 & 73.9 & 29 \\
\hline Andhra Pradesh & 56.1 & 70.3 & 14.2 \\
\hline NCT Delhi & 53.8 & 69.1 & 15.3 \\
\hline Himachal Pradesh & 49.4 & 67.2 & 17.8 \\
\hline West Bengal & 28.0 & 62.5 & 34.5 \\
\hline Mizoram & 53.6 & 61.9 & 8.3 \\
\hline Odisha & 36.5 & 60.8 & 24.3 \\
\hline Gujarat & 36.8 & 60.0 & 23.2 \\
\hline Dadar\& nagar haveli and Daman \& Diu & 42.3 & 59.8 & 17.5 \\
\hline Telangana & 52.7 & 57.9 & 5.2 \\
\hline Punjab & 42.6 & 55.4 & 12.8 \\
\hline Sikkim & 52.8 & 54.7 & 1.9 \\
\hline Manipur & 39.2 & 52.3 & 13.1 \\
\hline Madhya Pradesh & 23.5 & 51.4 & 27.9 \\
\hline Haryana & 32.5 & 51.2 & 18.7 \\
\hline Maharashtra & 40.6 & 48.2 & 7.6 \\
\hline Assam & 32.0 & 47.5 & 15.5 \\
\hline Uttarakhand & 24.9 & 46.6 & 21.7 \\
\hline Chhattisgarh & 30.3 & 45.0 & 14.7 \\
\hline Karnataka & 45.2 & 44.7 & -0.5 \\
\hline Meghalaya & 36.2 & 43.1 & 6.9 \\
\hline Rajasthan & 17.3 & 33.9 & 16.6 \\
\hline Jammu \& Kashmir & 30.2 & 29.8 & -0.4 \\
\hline Jharkhand & 15.3 & 28.2 & 12.9 \\
\hline Tripura & 13.4 & 26.7 & 13.3 \\
\hline Arunachal Pradesh & 8.3 & 23.8 & 15.5 \\
\hline Uttar Pradesh & 12.9 & 22.3 & 9.4 \\
\hline Bihar & 9.7 & 18.0 & 8.3 \\
\hline Ladakh & 29.5 & 14.3 & -15.2 \\
\hline Nagaland & 4.4 & 10.2 & 5.8 \\
\hline \hline
\end{tabular}

Mothers consumed IFA for 180 days

\begin{tabular}{|l|l|l}
\hline Mothers who consumed & \\
\hline
\end{tabular}

(StatesiUTs)!Indicators or more when they were
pregnant $(\%)$

\begin{tabular}{|l|r|r|r|}
\hline Round & NFHS 4 & NFHS 5 & Change \\
\hline
\end{tabular} \begin{tabular}{|l|r|r|r|}
\hline INDIA & 14.4 & 26.0 & 11.6 \\
\hline
\end{tabular}

\begin{tabular}{|r|r|r|r|}
\hline Kerala & 47.4 & 67.0 & 19.6 \\
\hline Sikkim \\
\hline Loa
\end{tabular}

\begin{tabular}{|l|r|r|r|}
\hline Goa & 52.8 & 65.0 & 12.2 \\
\hline
\end{tabular}

\begin{tabular}{|r|r|r|r|}
\hline Uducherry & 36.3 & 64.9 & 28.6 \\
\hline Odisha \\
\hline Dadar\& nagar haveli and Daman \& Diu
\end{tabular}

Candigarh

amil Nadu

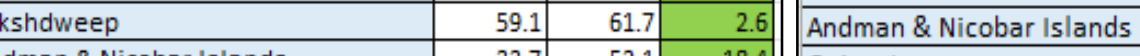

\begin{tabular}{|l|r|r|r|}
\hline & 33.7 & 52.1 & 18.4 \\
\hline Gujarat
\end{tabular}

\begin{tabular}{|l|r|r|r|}
\hline & 29.9 & 49.0 & 19.1 \\
\hline Chel Delhi & 18.5 & 43.2 & 24.7 \\
\hline Kerala
\end{tabular}

\begin{tabular}{|l|r|r|r|}
\hline imachal Pradesh & 18.5 & 43.2 & 24.7 \\
\hline Kerala \\
\hline Jammu \& Kashmir
\end{tabular}

\begin{tabular}{|l|r|r|r|}
\hline ndhra Pradesh & 30.6 & 41.1 & 10.5 \\
\hline
\end{tabular}

\begin{tabular}{|l|r|r|r}
\hline unjab & 19.9 & 40.5 & 20.6 \\
\hline Andhra Pradesh
\end{tabular}

\begin{tabular}{|l|r|r|r||}
\hline Dadar\& nagar haveli and Daman \& Diu & 26.2 & 36.2 & 10.0 \\
\hline Madhya Pradesh
\end{tabular}

\begin{tabular}{|l|r|r|r|}
\hline disha & 4.2 & 34.4 & 30.2 \\
\hline
\end{tabular}

Telangana

ladhya Pradesh

ikkim

Maharashtra

\begin{tabular}{|r|r|r|r|}
\hline West Bengal & 28.0 & 30.9 & 2.9 \\
\hline & 6.0 & 30.8 & 24.8 \\
\hline
\end{tabular}

\begin{tabular}{|l|r|r|r|}
\hline arnataka & 32.6 & 26.7 & -5.9 \\
\hline Chhattisgarh & 9.5 & 26.3 & 16.8 \\
\hline
\end{tabular}

Uttarakhand

Tegaland

eghal

ammu \& Kashmir

harkhand

Rajasthan

Mizoram

Bihar

Tripura

Arunachal Pradesh

Ladakh
| Mothers who consumed
Vitamin A dose received by children

Uttar Pradesh

Chandigarh

Telangana

Maharashtra

Jharkhand

Tripura

Arunachal Pradesh

West Bengal

Tamil Nadu

Mizoram

Rajasthan

Meghalaya

Assam

Bihar

Uttarakhand

NCT Delhi

Manipur

Lakshdweep

82.4

$-7$

\begin{tabular}{|r|r|}
\hline 76.0 & 86.0 \\
\hline 74.7 & 85.6 \\
\hline
\end{tabular}
(States:UTs)|Indicators

Children age 9-35months who recieved a vitamin $A$ dose in last 6 months_NFHS 5

\begin{tabular}{r|r|r|r|}
\hline & NFHS 4 & NFHS 5 & Change \\
\hline
\end{tabular}
\begin{tabular}{r|r|r|}
\hline 64.5 & 71.2 & 6.7 \\
\hline
\end{tabular}

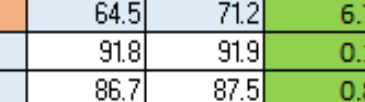
\begin{tabular}{|r|r|r|}
\hline 72.7 & 87.1 & 14.4 \\
\hline 75 & 87.1 & 11.5 \\
\hline
\end{tabular} \begin{tabular}{|l|l|l|}
\hline 75.6 & 87.1 & 11.5 \\
\hline 67.2 & 86.2 & 19. \\
\hline
\end{tabular} \begin{tabular}{|c|c|}
86.2 & 3.8 \\
\hline
\end{tabular} \begin{tabular}{r|r|}
86.0 & 10.0 \\
\hline 85.6 & 10.9 \\
\hline 84.5 & 7. \\
\hline
\end{tabular} \begin{tabular}{r|r|}
85.6 & 10.9 \\
\hline 84.5 & 7.8 \\
\hline
\end{tabular} BACK 


\section{Antenatal and Postnatal care}

\section{Mothers received an antenatal check up}

\begin{tabular}{|c|c|c|c|}
\hline (STATESUIs)]NNDICATORS & $\begin{array}{r}\text { Mothers w } \\
\text { chec } \\
\text { trim }\end{array}$ & $\begin{array}{l}\text { o had an } \\
\text {-up in the } \\
\text { ster_NFH }\end{array}$ & $\begin{array}{l}\text { antenatal } \\
\text { first } \\
\text { IS } 5\end{array}$ \\
\hline ROUND & NFHS 4 & NFHS 5 & Change \\
\hline INDIA & 58.6 & 70.0 & 11.4 \\
\hline Lakshadweep & 90.6 & 99.6 & 9.0 \\
\hline Kerala & 95.1 & 93.6 & -1.5 \\
\hline Telangana & 83.1 & 88.5 & 5.4 \\
\hline Jammu and Kashmir & 76.7 & 86.6 & 9.9 \\
\hline Ladakh & 79.5 & 85.8 & 6.3 \\
\hline Haryana & 63.2 & 85.2 & 22.0 \\
\hline Puducherry & 80.6 & 82.4 & 1.8 \\
\hline Chandigarh & 67.4 & 82.3 & 14.9 \\
\hline Andhra pradesh & 82.3 & 81.7 & -0.6 \\
\hline Manipur & 77.0 & 79.9 & 2.9 \\
\hline Gujarat & 73.8 & 79.3 & 5.5 \\
\hline Dadar\& nagar haveli and Daman \& Diu & 66.9 & 77.7 & 10.8 \\
\hline Tamil Nadu & 64.0 & 77.4 & 13.4 \\
\hline Andaman \& Nicobar & 68.4 & 77.1 & 8.7 \\
\hline Ddisha & 64.0 & 76.9 & 12.9 \\
\hline NCT Delhi & 63.0 & 76.4 & 13.4 \\
\hline Rajasthan & 63.0 & 76.3 & 13.3 \\
\hline Madhya Pradesh & 53.0 & 75.4 & 22.4 \\
\hline Mizoram & 65.6 & 72.7 & 7.1 \\
\hline West bengal & 54.9 & 72.6 & 17.7 \\
\hline Himachal pradesh & 70.5 & 72.4 & 1.9 \\
\hline Karnataka & 65.9 & 71.0 & 5.1 \\
\hline Maharastra & 67.6 & 70.9 & 3.3 \\
\hline Goa & 84.4 & 70.3 & -14.1 \\
\hline Uttarakhand & 53.5 & 68.8 & 15.3 \\
\hline Puniab & 75.6 & 68.5 & -7.1 \\
\hline Jharkhand & 52.0 & 68.0 & 16.0 \\
\hline Chattisgarh & 70.8 & 65.7 & -5.1 \\
\hline Assam & 55.1 & 63.8 & 8.7 \\
\hline Sikkim & 76.2 & 63.7 & -12.5 \\
\hline Tripura & 66.4 & 63.2 & -3.2 \\
\hline Uttar Pradesh & 45.9 & 62.5 & 16.6 \\
\hline Meghalaya & 53.5 & 53.9 & 0.4 \\
\hline Arunachal Pradesh & 36.9 & 53.1 & 16.2 \\
\hline BIHAR & 34.6 & 52.9 & 18.3 \\
\hline Nagaland & 24.7 & 49.5 & 24.8 \\
\hline
\end{tabular}

SDG 2030 target for mothers who had at least 4

Improvement in the indicator

Decline in the indicato

No change

States arranged as pe highest to lowest coverage (NFHS-5)

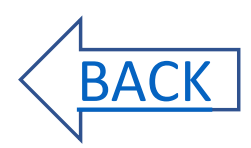
antenatal care visits: $75 \%$

\begin{tabular}{|l|r|r|r|}
\hline \multicolumn{1}{|c|}{$\quad$ (STATES/Uts)/INDICATORS } & \multicolumn{2}{|c|}{ Mothers who had at least 4 } \\
antenatal care visits_NFHS 5 \\
\hline ROUND & NFHS 4 & NFHS 5 & Change \\
\hline INDIA & 51.2 & 58.1 & 6.9 \\
\hline Goa & 89.0 & 93.0 & 4.0 \\
\hline Tamil Nadu & 81.1 & 89.9 & 8.8 \\
\hline Lakshadweep & 82.3 & 88.3 & 6.0 \\
\hline Puducherry & 87.7 & 86.9 & -0.8 \\
\hline Dadar\& nagar haveli and Daman \& Diu & 71.9 & 86.2 & 14.3 \\
\hline Andaman \& Nicobar & 92.1 & 83.4 & -8.7 \\
\hline Jammu and Kashmir & 81.2 & 80.9 & -0.3 \\
\hline Manipur & 69.0 & 79.4 & 10.4 \\
\hline Chandigarh & 64.5 & 78.7 & 14.2 \\
\hline Kerala & 90.1 & 78.6 & -11.5 \\
\hline Ladakh & 87.5 & 78.4 & -9.1 \\
\hline Odisha & 61.9 & 78.1 & 16.2 \\
\hline NCT Delhi & 67.9 & 77.2 & 9.3 \\
\hline Gujarat & 70.5 & 76.9 & 6.4 \\
\hline West bengal & 76.4 & 75.8 & -0.6 \\
\hline Karnataka & 70.1 & 70.9 & 0.8 \\
\hline Telangana & 74.9 & 70.4 & -4.5 \\
\hline Maharastra & 72.2 & 70.3 & -1.9 \\
\hline Himachal pradesh & 69.1 & 70.3 & 1.2 \\
\hline Andhra pradesh & 76.3 & 67.5 & -8.8 \\
\hline Uttarakhand & 30.9 & 61.8 & 30.9 \\
\hline Haryana & 45.1 & 60.4 & 15.3 \\
\hline Chattisgarh & 59.1 & 60.1 & 1.0 \\
\hline Punjab & 68.5 & 59.3 & -9.2 \\
\hline Sikkim & 74.7 & 58.4 & -16.3 \\
\hline Mizoram & 61.4 & 58.0 & -3.4 \\
\hline Madhya Pradesh & 35.7 & 57.5 & 21.8 \\
\hline Rajasthan & 38.5 & 55.3 & 16.8 \\
\hline Tripura & 64.3 & 52.7 & -11.6 \\
\hline Meghalaya & 50.0 & 52.2 & 2.2 \\
\hline Assam & 46.4 & 50.7 & 4.3 \\
\hline Uttar Pradesh & 26.4 & 42.4 & 16.0 \\
\hline Jharkhand & 30.3 & 38.6 & 8.3 \\
\hline Arunachal Pradesh & 26.7 & 36.5 & 9.8 \\
\hline BIHAR & 14.4 & 25.2 & 10.8 \\
\hline Nagaland & 15.0 & 20.7 & 5.7 \\
\hline
\end{tabular}

Mothers received postnatal check up

\begin{tabular}{|c|c|c|c|}
\hline (STATESUUts)|INDICATORS & $\begin{array}{l}\text { Mothers } \\
\text { care from } \\
\text { doctorlNu } \\
\text { her health } \\
\text { days of d }\end{array}$ & $\begin{array}{l}\text { hho recieve } \\
\text { a } \\
\text { sedLHVAN } \\
\text { personne } \\
\text { elivery }\end{array}$ & $\begin{array}{l}\text { sst natal } \\
\text { nidwifelot } \\
\text { hin two }\end{array}$ \\
\hline ROUIND & NFHS 4 & NFHS 5 & Change \\
\hline INDIA & 62.4 & 78.0 & 15.6 \\
\hline Goa & 92.1 & 95.4 & 3.3 \\
\hline Kerala & 88.7 & 93.3 & 4.6 \\
\hline Tamil Nadu & 74.0 & 93.2 & 19.2 \\
\hline Puducherry & 84.9 & 93.1 & 8.2 \\
\hline Lakshadweep & 92.7 & 92.6 & -0.1 \\
\hline Dadar\& nagar haveli and Daman \& Diu & 64.8 & 91.6 & 26.8 \\
\hline Haryana & 67.3 & 91.3 & 24.0 \\
\hline Andhra pradesh & 79.7 & 90.7 & 11.0 \\
\hline Chandigarh & 89.0 & 90.6 & 1.6 \\
\hline Gujarat & 63.3 & 89.7 & 26.4 \\
\hline Andaman \& Nicobar & 75.0 & 88.9 & 13.9 \\
\hline Ddisha & 73.2 & 88.4 & 15.2 \\
\hline Telangana & 81.7 & 87.6 & 5.9 \\
\hline Karnataka & 65.5 & 87.4 & 21.9 \\
\hline Himachal pradesh & 70.2 & 86.3 & 16.1 \\
\hline Puniab & 87.2 & 86.2 & -1.0 \\
\hline Maharastra & 78.5 & 85.4 & 6.9 \\
\hline NCT Delhi & 62.3 & 85.4 & 23.1 \\
\hline Rajasthan & 63.7 & 85.3 & 21.6 \\
\hline Jammu and Kashmir & 74.7 & 84.2 & 9.5 \\
\hline Chattisgarh & 63.6 & 84.0 & 20.4 \\
\hline Madhya Pradesh & 54.9 & 83.5 & 28.6 \\
\hline Ladakh & 83.3 & 79.6 & -3.7 \\
\hline Uttarakhand & 54.8 & 78.0 & 23.2 \\
\hline Manipur & 64.6 & 73.4 & 8.8 \\
\hline Uttar Pradesh & 54.0 & 72.0 & 18.0 \\
\hline Tripura & 62.1 & 71.9 & 9.8 \\
\hline Sikkim & 74.2 & 69.3 & -4.9 \\
\hline Jharkhand & 44.4 & 69.1 & 24.7 \\
\hline Mizoram & 64.5 & 68.0 & 3.5 \\
\hline West bengal & 61.1 & 68.0 & 6.9 \\
\hline Assam & 54.0 & 65.3 & 11.3 \\
\hline $\mathrm{BH} A \mathrm{~A}$ & 42.3 & 57.3 & 15.0 \\
\hline Arunachal Pradesh & 28.8 & 56.4 & 27.6 \\
\hline Meghalaya & 47.5 & 43.9 & -3.6 \\
\hline Nagaland & 22.3 & 43.9 & 21.6 \\
\hline
\end{tabular}




\section{Delivery care}

SDG 2030 target for Institutional births: $92 \%$

SDG target Achieved

SDG target Not Achieved

Improvement in the indicator

Decline in the indicator

No change

States arranged as per highest to lowest values for institutional births \& lowest to highest values for caesarean delivery (NFHS-5)

Institutional births: States arranged as per highest to lowest coverage in NFHS-5

Births delivered by Caesarean section: States arranged as per lowest to highest prevalence (NFHS-5)

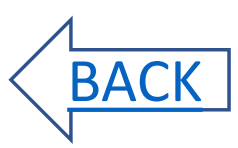

\begin{tabular}{|c|c|c|c|c|c|c|c|}
\hline \multirow{2}{*}{ 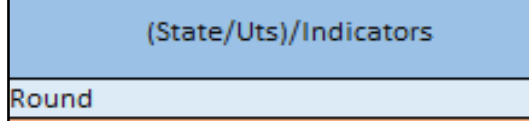 } & \multicolumn{3}{|c|}{ Institutional births_NFHS 5} & \multirow[t]{2}{*}{ (State/Uts)/Indicators } & \multicolumn{3}{|c|}{$\begin{array}{c}\text { Births delivered by caesarean } \\
\text { section_NFHS } 5\end{array}$} \\
\hline & NFHS 4 & NFHS 5 & Change & & NFHS 4 & NFHS 5 & Change \\
\hline INDIA & 78.9 & 88.6 & 9.7 & INDIA & 17.2 & 21.5 & 4.3 \\
\hline Kerala & 99.8 & 99.8 & 0.0 & Nagaland & 5.8 & 5.2 & -0.6 \\
\hline Goa & 96.9 & 99.7 & 2.8 & Meghalaya & 7.6 & 8.2 & 0.6 \\
\hline Tamil Nadu & 98.9 & 99.6 & 0.7 & BIHAR & 6.2 & 9.7 & 3.5 \\
\hline Lakshadweep & 99.3 & 99.6 & 0.3 & Rajasthan & 8.6 & 10.4 & 1.8 \\
\hline Puducherry & 99.9 & 99.6 & -0.3 & Mizoram & 12.7 & 10.8 & -1.9 \\
\hline Andaman \& Nicobar & 96.4 & 99 & 2.6 & Madhya Pradesh & 8.6 & 12.1 & 3.5 \\
\hline Telangana & 91.5 & 97 & 5.5 & harkhand & 9.9 & 12.8 & 2.9 \\
\hline Karnataka & 94 & 97 & 3.0 & Uttar Pradesh & 9.4 & 13.7 & 4.3 \\
\hline Chandigarh & 91.6 & 96.9 & 5.3 & Arunachal Pradesh & 8.9 & 14.8 & 5.9 \\
\hline Dadar\& nagar haveli and Daman \& Diu & 88.5 & 96.5 & 8.0 & Chattisgarh & 9.9 & 15.2 & 5.3 \\
\hline Andhra pradesh & 91.5 & 96.5 & 5.0 & Assam & 13.4 & 18.1 & 4.7 \\
\hline Ladakh & 90.8 & 95.1 & 4.3 & Haryana & 11.7 & 19.5 & 7.8 \\
\hline Haryana & 80.4 & 94.9 & 14.5 & Uttarakhand & 13.1 & 20.4 & 7.3 \\
\hline Rajasthan & 84 & 94.9 & 10.9 & Gujarat & 18.4 & 21.0 & 2.6 \\
\hline Maharastra & 90.3 & 94.7 & 4.4 & Himachal pradesh & 16.7 & 21.0 & 4.3 \\
\hline Sikkim & 94.7 & 94.7 & 0.0 & Odisha & 13.8 & 21.6 & 7.8 \\
\hline Gujarat & 88.5 & 94.3 & 5.8 & Dadar\& nagar haveli and Daman \& Diu & 16.1 & 22.9 & 6.8 \\
\hline Punjab & 90.5 & 94.3 & 3.8 & NCT Delhi & 26.7 & 23.6 & -3.1 \\
\hline lammu and Kashmir & 85.5 & 92.4 & 6.9 & Fripura & 20.5 & 25.1 & 4.6 \\
\hline Odisha & 85.3 & 92.2 & 6.9 & Maharastra & 20.1 & 25.4 & 5.3 \\
\hline NCT Delhi & 84.4 & 91.8 & 7.4 & Manipur & 21.1 & 25.6 & 4.5 \\
\hline West bengal & 75.2 & 91.7 & 16.5 & Andaman \& Nicobar & 19.3 & 29.9 & 10.6 \\
\hline Madhya Pradesh & 80.8 & 90.7 & 9.9 & |Lakshadweep & 38.4 & 31.3 & -7.1 \\
\hline Tripura & 79.9 & 89.2 & 9.3 & Chandigarh & 22.6 & 31.3 & 8.7 \\
\hline Himachal pradesh & 76.4 & 88.2 & 11.8 & Karnataka & 23.6 & 31.5 & 7.9 \\
\hline Mizoram & 79.7 & 85.8 & 6.1 & West bengal & 23.8 & 32.6 & 8.8 \\
\hline Chattisgarh & 70.2 & 85.7 & 15.5 & Sikkim & 20.9 & 32.8 & 11.9 \\
\hline Assam & 70.6 & 84.1 & 13.5 & |Puducherry & 33.6 & 36.3 & 2.7 \\
\hline Uttar Pradesh & 67.8 & 83.4 & 15.6 & |Ladakh & 16.1 & 37.6 & 21.5 \\
\hline Uttarakhand & 68.6 & 83.2 & 14.6 & Punjab & 24.6 & 38.5 & 13.9 \\
\hline Manipur & 69.1 & 79.9 & 10.8 & Kerala & 35.8 & 38.9 & 3.1 \\
\hline Arunachal Pradesh & 52.2 & 79.2 & 27.0 & Goa & $\begin{array}{l}31.4 \\
33.4\end{array}$ & 39.5 & 8.1 \\
\hline BIHAR & 63.8 & 76.2 & 12.4 & Andhra pradesh & $\begin{array}{ll}35.4 \\
40.1\end{array}$ & $\begin{array}{ll}41.7 \\
42.4\end{array}$ & $\frac{8.3}{2.3}$ \\
\hline harkhand & 61.9 & 75.8 & 13.9 & Tamil Nadu & 34.1 & 44.9 & $\begin{array}{r}2.3 \\
10.8\end{array}$ \\
\hline Meghalaya & 51.4 & 58.1 & 6.7 & Telangana & 57.7 & 60.7 & 3.0 \\
\hline
\end{tabular}




\section{Childhood diseases}

Prevalence of diarrhea

Improvement in the indicator

Decline in the indicator

No change

States arranged as per lowest to highest prevalence

(NFHS-5)

BACK

\begin{tabular}{|c|c|c|c|}
\hline \multirow{2}{*}{\begin{tabular}{|l} 
[Statelutsindicators] \\
BOUND \\
\end{tabular}} & \multicolumn{3}{|c|}{$\begin{array}{c}\text { Prevalence of diarchoea in the } 2 \text { weeks } \\
\text { preceding the survey_NFHS } 5\end{array}$} \\
\hline & NFHS4 & NFHS5 & CHANGE \\
\hline NDIA & 9.2 & 7.3 & \begin{tabular}{l|r}
3 & -1.9 \\
\end{tabular} \\
\hline akshadweep & 6.3 & 2.3 & -4.0 \\
\hline Padar\& nagar haveli and Daman \& Diu & 4.1 & 2.6 & -1.5 \\
\hline Goa & 3.8 & 3.2 & -0.6 \\
\hline Jagaland & 5.1 & 3.4 & -1.7 \\
\hline Dhhattisgarh & 9.1 & 3.6 & -5.5 \\
\hline Puducherry & 11.3 & 3.7 & -7.6 \\
\hline Tamil Nadu & 8.0 & 3.7 & -4.3 \\
\hline Ehandigarh & 4.6 & 4.3 & -0.3 \\
\hline Kerala & 3.4 & 4.3 & 0.5 \\
\hline Mizoram & 7.6 & 4.3 & -3.3 \\
\hline \begin{tabular}{|l|l} 
Ittarakhand \\
\end{tabular} & 17.0 & 4.4 & -12.6 \\
\hline Himachal pradesh & 6.6 & 4.7 & -1.9 \\
\hline Haryana & 7.7 & 4.9 & -2.8 \\
\hline Puniab & 6.6 & 4.9 & -1.7 \\
\hline Arunachal Pradesh & 6.5 & 5.1 & -1.4 \\
\hline Karnataka & 4.5 & 5.3 & 0.8 \\
\hline 4 ssam & 2.9 & 5.5 & 2.6 \\
\hline Bikkim & 1.8 & 5.5 & 3.7 \\
\hline Andaman \& Nicobar & 5.3 & 5.6 & 0.8 \\
\hline Jammu and Kashmir & 7.6 & 5.6 & -2.0 \\
\hline Manipur & 5.8 & 5.6 & -0.2 \\
\hline \begin{tabular}{|l} 
Ittar Pradesh \\
\end{tabular} & 15.0 & 5.6 & -9.4 \\
\hline iajasthan & 7.4 & 6.1 & -1.3 \\
\hline Tripura & 4.9 & 6.2 & 1.8 \\
\hline Madhya Pradesh & 9.5 & 6.4 & -3.1 \\
\hline West bengal & 5.9 & 6.5 & 0.6 \\
\hline Andhra pradesh & 6.6 & 7.2 & 0.6 \\
\hline Jharkhand & 6.9 & 7.2 & 0.8 \\
\hline Telangana & 8.2 & 7.4 & -0.8 \\
\hline Gujarat & 8.4 & 8.2 & -0.2 \\
\hline \begin{tabular}{|l|} 
adakh \\
\end{tabular} & 3.1 & 8.5 & 5. \\
\hline Maharastra & 8.5 & 8.9 & 0.4 \\
\hline pdisha & 9.8 & 9.7 & -0.1 \\
\hline Meghalaya & 10.6 & 10.4 & -0.2 \\
\hline NCT Delhi & 9.6 & 10.6 & 1.0 \\
\hline PHAR & 10.4 & 13.7 & 3.8 \\
\hline
\end{tabular}

Prevalence of acute respiratory infection (ARI)

\begin{tabular}{|c|c|c|c|}
\hline (State/Uts)/Indicators & $\begin{array}{l}\text { Prevalenc } \\
\text { acute res } \\
\text { (ARI) } \\
\text { preceding }\end{array}$ & $\begin{array}{l}\text { ice of sym } \\
\text { spiratory } \\
\text { in the } 2 \mathrm{v} \\
\text { the surve }\end{array}$ & $\begin{array}{l}\text { ptoms of } \\
\text { infection } \\
\text { veeks } \\
\text { ey_NFHS } 5\end{array}$ \\
\hline Round & NFHS 4 & NFHS 5 & CHANGE \\
\hline NDIA & 2.7 & $\begin{aligned} 2.8 \\
\end{aligned}$ & 0.1 \\
\hline Chandigarh & 2.8 & 0.3 & -2.5 \\
\hline Dadar\& nagar haveli and Daman \& Diu & 1.5 & 0.3 & -1.2 \\
\hline Mizoram & 2.2 & 0.6 & -1.6 \\
\hline Sikkim & 0.3 & 0.7 & 0.4 \\
\hline Goa & 1.4 & 0.9 & -0.5 \\
\hline Gujarat & 1.4 & 1 & -0.4 \\
\hline Nagaland & 1.4 & 1.1 & -0.3 \\
\hline Tamil Nadu & 2.8 & 1.1 & -1.7 \\
\hline Tripura & 2.6 & 1.3 & -1.3 \\
\hline Lakshadweep & 0.9 & 1.4 & 0.5 \\
\hline Chattisgarh & 2.2 & 1.5 & -0.7 \\
\hline Himachal pradesh & 1.6 & 1.5 & -0.1 \\
\hline Karnataka & 1.2 & 1.5 & 0.3 \\
\hline Andaman \& Nicobar & 1.5 & 1.7 & 0.2 \\
\hline Manipur & 1.7 & 1.8 & 0.1 \\
\hline Arunachal Pradesh & 2.1 & 2.1 & 0 \\
\hline harkhand & 3.2 & 2.1 & -1.1 \\
\hline Telangana & 2 & 2.2 & 0.2 \\
\hline \begin{tabular}{|l|} 
Haryana \\
\end{tabular} & 3.2 & 2.3 & -0.9 \\
\hline Uttarakhand & 4.6 & 2.3 & -2.3 \\
\hline Andhra pradesh & 0.5 & 2.4 & 1.9 \\
\hline Kerala & 0.8 & 2.4 & 1.6 \\
\hline Assam & 1 & 2.5 & 1.5 \\
\hline Punjab & 4.1 & 2.5 & -1.6 \\
\hline Madhya Pradesh & 2.1 & 2.6 & 0.5 \\
\hline West bengal & 3.3 & 2.8 & -0.5 \\
\hline Rajasthan & 2.1 & 2.9 & 0.8 \\
\hline Maharastra & 2.4 & 3.2 & 0.8 \\
\hline Odisha & 2.4 & 3.2 & 0.8 \\
\hline Bihar & 2.5 & 3.5 & \\
\hline Uttar Pradesh & 4.7 & 3.5 & -1.2 \\
\hline lammu and Kashmir & 5.5 & 3.9 & -1.6 \\
\hline Meghalaya & 5.8 & 4.8 & -1 \\
\hline Puducherry & \begin{tabular}{l|l}
3 \\
\end{tabular} & 4.9 & 1.9 \\
\hline adakh & 1 & 5.3 & 4.3 \\
\hline NCT Delhi & 2.4 & 5.6 & 3.2 \\
\hline
\end{tabular}


Treatment for childhood diseases

Children with diarrhea received ORS

\begin{tabular}{|c|c|c|c|}
\hline (Statedutsinndicator) & \multicolumn{3}{|c|}{\begin{tabular}{|l} 
Children with diarchoea in the \\
2 weeks preceding the survey \\
who received oral rehydration \\
salts (DRS)_NFHS 5
\end{tabular}} \\
\hline ROUND & NFHS 4 & NFHS5 & CHANGE \\
\hline INDIA & 50.6 & 60.6 & 10.0 \\
\hline Chandigarh & na & na & na \\
\hline Dadar\& nagar haveli and Daman \& Diu & 84.9 & na & na \\
\hline Goa & na & na & na \\
\hline Lakshadweep & na & na & na \\
\hline Puducherry & 71.2 & na & na \\
\hline Jammu and Kashmir & 69.1 & 80.8 & 11.7 \\
\hline \begin{tabular}{|l} 
Ladakh \\
\end{tabular} & na & 78.3 & na \\
\hline West bengal & 64.7 & 75.3 & 10.6 \\
\hline Himachal pradesh & 62.7 & 73.7 & 11.0 \\
\hline Meghalaya & 77.4 & 73.2 & -4.2 \\
\hline Mizoram & 70.0 & 71.4 & 1.4 \\
\hline Karnataka & 52.8 & 71.3 & 18.5 \\
\hline Manipur & 60.2 & 69.8 & 9.6 \\
\hline Assam & 51.9 & 69.1 & 17.2 \\
\hline Chhattisgarh & 67.9 & 67.3 & -0.6 \\
\hline Tripura & 46.3 & 67.2 & 20.9 \\
\hline Gujarat & 46.2 & 66.5 & 20.3 \\
\hline Ddisha & 68.6 & 65.7 & -2.9 \\
\hline Madhya Pradesh & 55.2 & 65.2 & 10.0 \\
\hline Andaman \& Nicobar & 65.0 & 65.0 & 0.0 \\
\hline NCT Delhi & 62.1 & 64.5 & 2.4 \\
\hline Rajasthan & 56.2 & 64.3 & 8.1 \\
\hline Sikkim & na & 64.2 & na \\
\hline Arrunachal Pradesh & 66.1 & 62.7 & -3.4 \\
\hline Andhra pradesh & 47.6 & 62.5 & 14.9 \\
\hline Kerala & 49.4 & 61.1 & 11.7 \\
\hline Punjab & 66.2 & 60.7 & -5.5 \\
\hline Maharastra & 60.5 & 59.5 & -10 \\
\hline BHAR & 45.2 & 58.2 & 13.0 \\
\hline Telangana & 56.8 & 56.3 & -0.5 \\
\hline $\mid$ IIttarakhand & 56.0 & 55.9 & -0.1 \\
\hline Jharkhand & 44.8 & 55.6 & 10.8 \\
\hline Nagaland & 40.3 & 54.5 & 14.2 \\
\hline Tamil Nadu & 61.8 & 53.8 & -8.0 \\
\hline 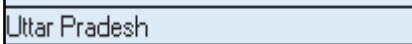 & 37.9 & 50.7 & 12.8 \\
\hline Haryana & 60.6 & 46.6 & -14.0 \\
\hline
\end{tabular}

\section{Children with diarrhea received Zinc}

Improvement in the indicator

Decline in the indicator

No change

States arranged as per highest to lowest coverage (NFHS-5)

Children with diarrhea received zinc \& Children with diarrhea received ORS:

Data for Ladakh (in NFHS 5) \&

Andaman \& Nicobar (in NFHS 4 \& 5) is

based on 25-49 unweighted cases

Children with ARI taken to a health

facility: Data for Ladakh \&

Lakshadweep (in NFHS 4) is based on

25-49 unweighted cases

\section{$\langle\stackrel{\mathrm{BACK}}{\mathrm{NEXT}\rangle}$}

Children with ARI taken to health facility

\begin{tabular}{|c|c|c|c|}
\hline [StatelUtsindicator] & $\begin{array}{r}\text { Children 1 } \\
\text { weeks pre } \\
\text { recei }\end{array}$ & $\begin{array}{l}\text { h diarrhoea in } \\
\text { ing the surve } \\
\text { zinc_NFHS }\end{array}$ & $\begin{array}{l}n \text { the } 2 \\
\text { ey who } \\
5\end{array}$ \\
\hline ROUNND & NFHS4 & NFHS5 & Change \\
\hline INDIA & 20.3 & 30.5 & 10.2 \\
\hline Chandigarh & na & na & $\mathrm{na}$ \\
\hline Dadar\& nagar haveli and Daman \& Diu & 12.9 & na & na \\
\hline Goa & na & na & na \\
\hline akshadweep & $\mathrm{na}$ & na & na \\
\hline Puducherry & 69.6 & na & na \\
\hline Ladakh & na & 54.5 & $\mathrm{na}$ \\
\hline Jammu and Kashmir & 39.3 & 50.5 & 11.2 \\
\hline Sikkim & na & 50.0 & na \\
\hline Karnataka & 34.3 & 45.5 & 11.2 \\
\hline Andaman \& Nicobar & 8.3 & 44.1 & 35.8 \\
\hline Andhra pradesh & 30.1 & 41.8 & 11.7 \\
\hline Meghalaya & 57.8 & 40.5 & -17.2 \\
\hline Chhattisgarh & 28.9 & 40.0 & 11.1 \\
\hline Telangana & 31.6 & 38.5 & 6.9 \\
\hline disha & 17.0 & 36.9 & 19.9 \\
\hline West bengal & 20.8 & 36.0 & 15.2 \\
\hline Madhya Pradesh & 26.6 & 35.6 & 9.0 \\
\hline Gujarat & 17.4 & 35.4 & 18.0 \\
\hline NCT Delhi & 25.3 & 32.8 & 7.5 \\
\hline Uttarakhand & 30.3 & 30.1 & -0.2 \\
\hline Mizoram & 29.0 & 29.8 & 0.8 \\
\hline Jharkhand & 19.1 & 28.9 & 9.8 \\
\hline Tamil Nadu & 41.3 & 28.9 & -12.4 \\
\hline Ittar Pradesh & 12.6 & 28.5 & 15.9 \\
\hline Assam & 22.0 & 28.0 & 6.0 \\
\hline Arunachal Pradesh & 35.8 & 27.9 & -7.5 \\
\hline Maharastra & 13.0 & 27.3 & 14.3 \\
\hline Rajasthan & 17.5 & 27.2 & 9.7 \\
\hline Puniab & 26.7 & 27.0 & 0.3 \\
\hline Haryana & 21.9 & 26.2 & 4.3 \\
\hline Manipur & 24.1 & 26.1 & 12.0 \\
\hline BHAR & 20.1 & 25.6 & 5.5 \\
\hline Kerala & 14.1 & 22.4 & 8.3 \\
\hline Himachal pradesh & 15.0 & 19.5 & 4.5 \\
\hline Tripura & 19.1 & 16.7 & -2. \\
\hline Nagaland & 16.0 & 9.1 & -6.5 \\
\hline
\end{tabular}

\begin{tabular}{|c|c|c|c|}
\hline \multirow{2}{*}{ 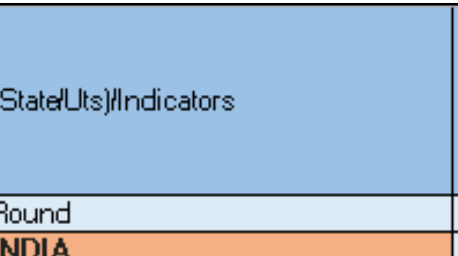 } & \multicolumn{3}{|c|}{$\begin{array}{l}\text { Children with fever or } \\
\text { symptoms of ARF in the } 2 \\
\text { weeks preceding the survey } \\
\text { taken to a health facitity or } \\
\text { health provider_NFHS } 5\end{array}$} \\
\hline & NFHS 4 & NFHS 5 & CHANGE \\
\hline & 73.2 & 69 & \\
\hline handigarh & na & na & na \\
\hline akshadweep & 86.5 & na & $\mathrm{na}$ \\
\hline adar\& nagar haveli and Daman \& Dic & 77.5 & 90.7 & 13.2 \\
\hline & 89 & 86.6 & \\
\hline erala & 90.1 & 86.2 & \\
\hline aharastra & 84.7 & 77.5 & -7 \\
\hline CT Delhi & 81.4 & 76.7 & -4.7 \\
\hline imachal pradesh & 78.4 & 76.2 & \\
\hline ujarat & 70.2 & 75.2 & $\frac{-2.2}{5.0}$ \\
\hline langana & 76.3 & 74.8 & \\
\hline aryana & 80.1 & 73.5 & \\
\hline leghalaya & 74.9 & 72.9 & \\
\hline ndaman \& Nicobar & 75.8 & 72.7 & -3 \\
\hline jest bengal & 73.5 & 71.3 & -2 \\
\hline jiasthan & 82.6 & 71.1 & -11. \\
\hline tharakhand & 79 & 71 & -8.1 \\
\hline ndhra pradesh & 77.3 & 70.2 & \\
\hline har & 59.8 & 69.4 & 9.6 \\
\hline amil Nadu & 82.2 & 67.4 & \\
\hline arnataka & 76.9 & 65.7 & \\
\hline Idisha & 72.9 & 65.4 & \\
\hline adhya Pradesh & 70.9 & 64.3 & -6.6 \\
\hline uducherry & 74 & 64.3 & -9. \\
\hline ripura & 73 & 64.2 & -8 \\
\hline hattisgarh & 70.1 & 63.6 & -6 \\
\hline tar Pradesh & 71.3 & 63 & -8 \\
\hline mmmu and Kashmir & 78.5 & 62.3 & -16.2 \\
\hline harkhand & 67.2 & 59.8 & -7. \\
\hline kkim & 63.8 & 59.5 & -4 \\
\hline adakh & 74.8 & 57.3 & -1.5 \\
\hline unjab & 90.3 & 57.3 & \\
\hline izoram & 50.1 & 53 & 2.9 \\
\hline ssam & 46.8 & 51.2 & 2.4 \\
\hline runachal Pradesh & 37.5 & 47.1 & 9.6 \\
\hline lanipur & 39.1 & 41.2 & 2.1 \\
\hline agaland & 31.3 & 30.9 & \\
\hline
\end{tabular}




\section{Children fully immunized}

\section{SDG 2030 target for Children aged 12-23 months fully vaccinated: 90\%}

SDG target Achieved

SDG target Not Achieved

Improvement in the indicator

Decline in the indicator

No change

States arranged as per highest to lowest coverage (NFHS-5)

Children fully immunized:

Data for Ladakh (in NFHS 4 \& 5) is based

on 25-49 unweighted cases

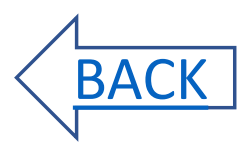

\begin{tabular}{|c|c|c|c|}
\hline [Statedபts]) Indicators & $\begin{array}{l}\text { Children } \\
\text { vaccinated } \\
\text { from eith }\end{array}$ & $\begin{array}{l}\text { age } 12-23 \\
\text { based or } \\
\text { ier vaccina } \\
\text { nother's re }\end{array}$ & $\begin{array}{l}\text { months fully } \\
\text { n information } \\
\text { ation card or } \\
\text { ecall }\end{array}$ \\
\hline Round & \begin{tabular}{l|l}
$\mathrm{NFHS} 4$ \\
\end{tabular} & NFHS 5 & Change \\
\hline INDIA & 62.0 & 76.4 & 14.4 \\
\hline Dadar\& nagar haveli and Daman \& Dil & 50.5 & 94.9 & 44.4 \\
\hline Odisha & 78.6 & 90.5 & 11.9 \\
\hline Himachal pradesh & 69.5 & 89.3 & 19.8 \\
\hline Tamil Nadu & 69.7 & 89.2 & 19.5 \\
\hline Ladakh & 81.9 & 88.2 & 6.3 \\
\hline West bengal & 84.4 & 87.8 & 3.4 \\
\hline Jammu and Kashmir & 75.0 & 86.4 & 11.4 \\
\hline Lakshadweep & 89.0 & 86.1 & -2.9 \\
\hline Karnataka & 62.6 & 84.1 & 21.5 \\
\hline Puducherry & 91.3 & 82.0 & -9.3 \\
\hline Goa & 88.4 & 81.9 & -6.5 \\
\hline Chandigarh & 79.5 & 80.9 & 1.4 \\
\hline Uttarakhand & 57.6 & 80.8 & 23.2 \\
\hline Sikkim & 83.0 & 80.6 & -2.4 \\
\hline Rajasthan & 54.8 & 80.4 & 25.6 \\
\hline Chattisgarh & 76.4 & 79.7 & 3.3 \\
\hline Telangana & 67.5 & 79.1 & 11.6 \\
\hline Andaman \& Nicobar & 73.2 & 77.8 & 4.6 \\
\hline Kerala & 82.1 & 77.8 & -4.3 \\
\hline Madhya Pradesh & 53.6 & 77.1 & 23.5 \\
\hline Haryana & 62.2 & 76.9 & 14.7 \\
\hline Gujarat & 50.4 & 76.3 & 25.9 \\
\hline Puniab & 89.1 & 76.2 & -12.9 \\
\hline NCT Delhi & 68.8 & 76.0 & 7.2 \\
\hline Jharkhand & 61.9 & 73.9 & 12.0 \\
\hline Maharastra & 56.2 & 73.5 & 17.3 \\
\hline Andhra pradesh & 65.3 & 73.0 & 7.7 \\
\hline Mizoram & 50.7 & 72.5 & 21.8 \\
\hline Bihar & 61.7 & 71.0 & 9.3 \\
\hline Uttar Pradesh & 51.1 & 69.6 & 18.5 \\
\hline Tripura & 54.5 & 69.5 & 15.0 \\
\hline Manipur & 65.8 & 68.8 & 3.0 \\
\hline Assam & 47.1 & 66.4 & 19.3 \\
\hline Arunachal Pradesh & 38.2 & 64.9 & 26.7 \\
\hline Meghalaya & 61.4 & 63.8 & 2.4 \\
\hline Nagaland & 35.4 & 57.9 & 22.5 \\
\hline
\end{tabular}




\section{Breastfeeding practices}

\section{Children under $\mathbf{3}$ years breastfed within one hour of birth}

WHA target Achieved

WHA target Not Achieved

Improvement in the indicator

Decline in the indicator

No change

States arranged as per highest to lowest coverage (NFHS-5)

For Exclusive breastfeeding: Data for Lakshadweep \& Goa (in NFHS 4 \& 5) is based on 250-499 unweighted person-years of exposure to the risk of death

\section{$\langle$ BACK NEXT $\rangle$}

\begin{tabular}{|c|c|c|c|}
\hline (States/UTs)/Indicators & $\begin{array}{l}\text { Children } \\
\text { breastfec }\end{array}$ & $\begin{array}{l}\text { nder age } \\
\text { within or }\end{array}$ & $\begin{array}{l}3 \text { years } \\
\text { le hour }\end{array}$ \\
\hline Round & NFHS 4 & NFHS 5 & Change \\
\hline INDIA & 41.6 & 41.8 & 0.2 \\
\hline Meghalaya & 60.6 & 78.8 & 18.2 \\
\hline Lakshdweep & 57.7 & 76.3 & 18.6 \\
\hline Odisha & 68.5 & 68.5 & 0.0 \\
\hline Kerala & 64.3 & 66.7 & 2.4 \\
\hline Chandigarh & 33.5 & 63.7 & 30.2 \\
\hline Goa & 73.3 & 61.6 & -11.7 \\
\hline Tamil Nadu & 54.7 & 60.2 & 5.5 \\
\hline Mizoram & 70.3 & 60.1 & -10.2 \\
\hline West Bengal & 47.4 & 59.4 & 12.0 \\
\hline Ladakh & 60.0 & 57.9 & -2.1 \\
\hline Nagaland & 53.1 & 57.9 & 4.8 \\
\hline Jammu \& Kashmir & 45.7 & 55.6 & 9.9 \\
\hline Puducherry & 65.3 & 54.1 & -11.2 \\
\hline Manipur & 65.4 & 53.7 & -11.7 \\
\hline Maharashtra & 57.5 & 53.2 & -4.3 \\
\hline Punjab & 30.7 & 53.1 & 22.4 \\
\hline Andhra Pradesh & 40.0 & 52.0 & 12.0 \\
\hline Arunachal Pradesh & 58.6 & 52.0 & -6.6 \\
\hline NCT Delhi & 28.0 & 51.2 & 23.2 \\
\hline Assam & 64.4 & 49.1 & -15.3 \\
\hline Karnataka & 56.3 & 49.1 & -7.2 \\
\hline Andman \& Nicobar Islands & 41.9 & 46.9 & 5.0 \\
\hline Himachal Pradesh & 41.1 & 45.1 & 4.0 \\
\hline Haryana & 42.4 & 41.6 & -0.8 \\
\hline Madhya Pradesh & 34.4 & 41.3 & 6.9 \\
\hline Uttarakhand & 27.8 & 41.3 & 13.5 \\
\hline Rajasthan & 28.4 & 40.7 & 12.3 \\
\hline Gujarat & 49.9 & 37.8 & -12.1 \\
\hline Telangana & 36.9 & 37.1 & 0.2 \\
\hline Tripura & 44.4 & 36.4 & -8.0 \\
\hline Sikkim & 66.5 & 33.0 & -33.5 \\
\hline Chhattisgarh & 47.1 & 32.2 & -14.9 \\
\hline Bihar & 34.9 & 31.1 & -3.8 \\
\hline Dadar\& nagar haveli and Daman \& Diu & 50.0 & 25.9 & -24.1 \\
\hline Uttar Pradesh & 25.2 & 23.9 & -1.3 \\
\hline Jharkhand & 33.1 & 21.5 & -11.6 \\
\hline
\end{tabular}

WHA 2025 target for exclusive breastfeeding: 69\%

\begin{tabular}{|c|c|c|c|}
\hline \multirow{2}{*}{$\begin{array}{l}\text { (States/UTs)/Indicators } \\
\text { Round }\end{array}$} & \multicolumn{3}{|c|}{$\begin{array}{l}\text { Children under age } 6 \\
\text { months exclusively } \\
\text { breastfed16 (\%) }\end{array}$} \\
\hline & NFHS 4 & NFHS 5 & Change \\
\hline INDIA & 54.9 & 63.7 & 8.8 \\
\hline Chandigarh & na & na & $\mathrm{na}$ \\
\hline Chhattisgarh & 77.2 & 80.3 & 3.1 \\
\hline Dadar\& nagar haveli and Daman \& Diu & 67.9 & 79.4 & 11.5 \\
\hline Jharkhand & 64.8 & 76.1 & 11.3 \\
\hline Madhya Pradesh & 58.2 & 74.0 & 15.8 \\
\hline Andman \& Nicobar Islands & 66.8 & 73.3 & 6.5 \\
\hline Odisha & 65.6 & 72.9 & 7.3 \\
\hline Maharashtra & 56.6 & 71.0 & 14.4 \\
\hline Ladakh & 64.1 & 70.9 & 6.8 \\
\hline Manipur & 73.6 & 70.7 & -2.5 \\
\hline Rajasthan & 58.2 & 70.4 & 12.2 \\
\hline Himachal Pradesh & 67.2 & 69.9 & 2.7 \\
\hline Haryana & 50.3 & 69.5 & 19.2 \\
\hline Telangana & 67.0 & 68.2 & 1.2 \\
\hline Andhra Pradesh & 70.2 & 68.0 & -2.2 \\
\hline Mizoram & 61.1 & 67.9 & 6.8 \\
\hline Lakshdweep & 54.8 & 67.0 & 12.2 \\
\hline Gujarat & 55.8 & 65.0 & 9.2 \\
\hline Puducherry & 45.5 & 64.8 & 19.3 \\
\hline NCT Delhi & 49.6 & 64.3 & 14.7 \\
\hline Assam & 63.5 & 63.6 & 0.1 \\
\hline Arunachal Pradesh & 57.0 & 63.4 & 6.4 \\
\hline Tripura & 70.7 & 62.1 & -8.6 \\
\hline Jammu \& Kashmir & 65.4 & 62.0 & -3.4 \\
\hline Goa & 60.9 & 61.4 & 0.5 \\
\hline Karnataka & 54.2 & 61.0 & 6.8 \\
\hline Uttar Pradesh & 41.6 & 59.7 & 18.1 \\
\hline Bihar & 53.4 & 58.9 & 5.5 \\
\hline Kerala & 53.3 & 55.5 & 2.2 \\
\hline Punjab & 53.0 & 55.5 & 2.5 \\
\hline Tamil Nadu & 48.3 & 55.1 & 6.8 \\
\hline West Bengal & 52.3 & 53.3 & 1 \\
\hline Uttarakhand & 51.2 & 52.5 & 1.3 \\
\hline Nagaland & 44.3 & 43.2 & -1.1 \\
\hline Meghalaya & 35.8 & 42.7 & 6.9 \\
\hline Sikkim & 54.6 & 28.3 & -26.3 \\
\hline
\end{tabular}




\section{Complementary feeding practices}

Improvement in the indicator

Decline in the indicator No change

States arranged as per highest to lowest coverage (NFHS-5)

Child receiving semi-solid foods and breastmilk:

Data for Ladakh (in NFHS 4) is based on 25-49 unweighted cases

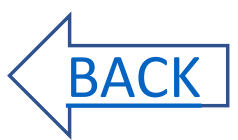

Children receiving semi solid food and breastmilk

\begin{tabular}{|c|c|c|c|}
\hline (States/UTs)/Indicators & $\begin{array}{l}\text { Childre } \\
\text { receiving } \\
\text { food and }\end{array}$ & $\begin{array}{l}\text { age } 6-8 \mathrm{n} \\
\text { olid or se } \\
\text { oreastmi }\end{array}$ & $\begin{array}{l}\text { onths } \\
\text { mi-solid } \\
\text { k16 (\%) }\end{array}$ \\
\hline Round & NFHS 4 & NFHS 5 & Change \\
\hline INDIA & 42.7 & 45.9 & 3.2 \\
\hline Andman \& Nicobar Islands & 45.1 & na & na \\
\hline Chandigarh & $\mathrm{na}$ & na & $\mathrm{na}$ \\
\hline Goa & na & na & $\mathrm{na}$ \\
\hline Ladakh & 42.2 & na & na \\
\hline Lakshdweep & na & na & na \\
\hline Manipur & 78.8 & 78.9 & 0.1 \\
\hline Kerala & 63.1 & 71.3 & 8.2 \\
\hline Nagaland & 70.7 & 69.4 & -1.3 \\
\hline Himachal Pradesh & 52.9 & 68.3 & 15.4 \\
\hline West Bengal & 52.0 & 67.8 & 15.8 \\
\hline Odisha & 54.9 & 67.5 & 12.6 \\
\hline Meghalaya & 67.4 & 66.8 & -0.6 \\
\hline Tamil Nadu & 67.5 & 66.5 & -1 \\
\hline Puducherry & 76.8 & 65.6 & -11.2 \\
\hline NCT Delhi & 35.4 & 62.9 & 27.5 \\
\hline Sikkim & 61.8 & 57.4 & -4.4 \\
\hline Mizoram & 68.2 & 56.9 & -11.3 \\
\hline Tripura & 13.6 & 53.1 & 39.5 \\
\hline Maharashtra & 43.3 & 52.7 & 9.4 \\
\hline Assam & 49.9 & 51.7 & 1.8 \\
\hline Telangana & 57.5 & 51.3 & -6.2 \\
\hline Andhra Pradesh & 56.1 & 50.8 & -5.3 \\
\hline Uttarakhand & 46.7 & 50.6 & 3.9 \\
\hline Arunachal Pradesh & 53.9 & 48.4 & -5.5 \\
\hline Punjab & 41.1 & 46.2 & 5.1 \\
\hline Karnataka & 46.0 & 45.8 & -0.2 \\
\hline Dadar\& nagar haveli and Daman \& Diu & 19.6 & 43.3 & 23.7 \\
\hline Haryana & 35.9 & 43.0 & 7.1 \\
\hline Gujarat & 49.4 & 42.0 & -7.4 \\
\hline Jammu \& Kashmir & 50.2 & 41.8 & -8.4 \\
\hline Chhattisgarh & 53.9 & 41.3 & -12.6 \\
\hline Madhya Pradesh & 38.1 & 39.5 & 1.4 \\
\hline Bihar & 30.8 & 39.0 & 8.2 \\
\hline Jharkhand & 47.2 & 38.8 & -8.4 \\
\hline Rajasthan & 30.1 & 38.0 & 7.9 \\
\hline Uttar Pradesh & 32.6 & 31.0 & -1.6 \\
\hline
\end{tabular}

Children receiving adequate diet

\begin{tabular}{|c|c|c|c|}
\hline (States/UTs)/Indicators & $\begin{array}{l}\text { Total chil } \\
\text { months r } \\
\text { adequate }\end{array}$ & $\begin{array}{l}\text { ren age } \\
\text { eiving a } \\
\text { liet } 16,1\end{array}$ & \\
\hline Round & NFHS 4 & NFHS 5 & Change \\
\hline INDIA & 8.7 & 11.1 & 2.4 \\
\hline Meghalaya & 23.5 & 29.8 & 6.3 \\
\hline Sikkim & 23.1 & 24.7 & 1.6 \\
\hline Ladakh & 23.9 & 24.0 & 0.1 \\
\hline Kerala & 21.4 & 23.5 & 2.1 \\
\hline West Bengal & 19.6 & 23.4 & 3.8 \\
\hline Puducherry & 31.1 & 22.9 & -8.2 \\
\hline Arunachal Pradesh & 14.0 & 22.0 & 8.0 \\
\hline Goa & 10.4 & 21.5 & 11.1 \\
\hline Odisha & 8.5 & 20.4 & 11.9 \\
\hline Manipur & 18.8 & 19.6 & 0.8 \\
\hline Andman \& Nicobar Islands & 14.2 & 19.5 & 5.3 \\
\hline Chandigarh & 0.0 & 19.0 & 19.0 \\
\hline Himachal Pradesh & 10.9 & 19.0 & 8.1 \\
\hline Lakshdweep & 15.9 & 19.0 & 3.1 \\
\hline NCT Delhi & 5.2 & 16.8 & 11.6 \\
\hline Tamil Nadu & 30.7 & 16.3 & -14.4 \\
\hline Nagaland & 18.8 & 14.5 & -4.3 \\
\hline Jammu \& Kashmir & 23.5 & 13.6 & -9.9 \\
\hline Tripura & 5.9 & 13.5 & 7.6 \\
\hline Mizoram & 14.5 & 13.4 & -1.1 \\
\hline Karnataka & 8.2 & 12.8 & 4.6 \\
\hline Uttarakhand & 8.5 & 12.5 & 4.0 \\
\hline Punjab & 5.9 & 11.9 & 6.0 \\
\hline Haryana & 7.5 & 11.8 & 4.3 \\
\hline Bihar & 7.5 & 10.9 & 3.4 \\
\hline Jharkhand & 7.2 & 10.5 & 3.3 \\
\hline Dadar\& nagar haveli and Daman \& Diu & 2.1 & 10.2 & 8.1 \\
\hline Andhra Pradesh & 7.6 & 9.3 & 1.7 \\
\hline Chhattisgarh & 10.9 & 9.3 & -1.6 \\
\hline Madhya Pradesh & 6.6 & 9.2 & 2.6 \\
\hline Telangana & 10.1 & 9.2 & -0.9 \\
\hline Maharashtra & 6.5 & 9.0 & 2.5 \\
\hline Rajasthan & 3.4 & 8.3 & 4.9 \\
\hline Assam & 8.9 & 8.0 & -0.9 \\
\hline Uttar Pradesh & 5.3 & 6.1 & 0.8 \\
\hline Gujarat & 5.2 & 5.9 & 0.7 \\
\hline
\end{tabular}


Household \& population profile-household aspects

Improvement in the indicator

Decline in the indicator

No change

States arranged as per highest to lowest coverage (NFHS-5)

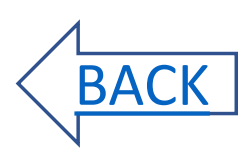

\begin{tabular}{|c|c|c|c|}
\hline \multicolumn{4}{|c|}{ Households with electricity } \\
\hline (StatestUts)|Indicators & $\begin{array}{l}\text { Populatio } \\
\text { with }\end{array}$ & $\begin{array}{l}\text { In living in } \\
\text { electricity_ }\end{array}$ & $\begin{array}{l}\text { households } \\
\text { JFHS } 5\end{array}$ \\
\hline ROUND & NFHS 4 & \begin{tabular}{|l|} 
NFHS 5 \\
\end{tabular} & CHANGE \\
\hline INDIA & 88 & 96.8 & 8.8 \\
\hline Goa & 99.8 & 100 & 0.2 \\
\hline Chandigarh & 99.6 & 99.9 & 0.3 \\
\hline NCT Delhi & 99.8 & 99.9 & 0.1 \\
\hline Puducherry & 99.8 & 99.9 & 0.1 \\
\hline Lakshadweep & 100 & 99.8 & -0.2 \\
\hline Dadar\& nagar haveli and Daman \& Diu & 98.3 & 99.7 & 1.4 \\
\hline Puniab & 99.6 & 99.7 & 0.1 \\
\hline Haryana & 98.9 & 99.6 & 0.7 \\
\hline Kerala & 99.2 & 99.6 & 0.4 \\
\hline Telangana & 98.9 & 99.6 & 0.7 \\
\hline Uttarakhand & 97.8 & 99.6 & 1.8 \\
\hline Andhra pradesh & 99.2 & 99.5 & 0.3 \\
\hline Himachal pradesh & 99.5 & 99.5 & 0.0 \\
\hline Ladakh & 98.7 & 99.5 & 0.8 \\
\hline Jammu and Kashmir & 97.2 & 99.3 & 2.1 \\
\hline Sikkim & 99.4 & 99.3 & -0.1 \\
\hline Tamil Nadu & 99 & 99.3 & 0.3 \\
\hline Karnataka & 98.3 & 99.1 & 0.8 \\
\hline Chattisgarh & 96.3 & 98.8 & 2.5 \\
\hline Nagaland & 96.7 & 98.6 & 1.9 \\
\hline Madhya Pradesh & 90.9 & 98.4 & 7.5 \\
\hline Mizoram & 96 & 98.2 & 2.2 \\
\hline Tripura & 92.8 & 98.2 & 5.4 \\
\hline Manipur & 92.8 & 98.1 & 5.3 \\
\hline Rajasthan & 91.2 & 98.1 & 6.9 \\
\hline Maharastra & 93.5 & 97.8 & 4.3 \\
\hline Andaman \& Nicobar & 97.2 & 97.6 & 0.4 \\
\hline Gujarat & 96.2 & 97.6 & 1.4 \\
\hline West bengal & 94.3 & 97.5 & 3.2 \\
\hline Odisha & 86.6 & 97 & 10.4 \\
\hline $\mathrm{BH} A \mathrm{R}$ & 60 & 96.3 & 36.3 \\
\hline Arunachal Pradesh & 88.3 & 94.8 & 6.5 \\
\hline Jharkhand & 81.2 & 94.3 & \begin{tabular}{r|r}
13.1 \\
\end{tabular} \\
\hline Assam & 78.3 & 92.6 & 14.3 \\
\hline Meghalaya & 91.8 & 91.9 & 0.1 \\
\hline Uttar Pradesh & 72.6 & 91 & 18.4 \\
\hline
\end{tabular}

Households with improved drinking water source

\begin{tabular}{|c|c|c|c|}
\hline \multirow{2}{*}{\begin{tabular}{|l} 
(States Ults) Indicator \\
ROUND \\
\end{tabular}} & \multicolumn{3}{|c|}{$\begin{array}{l}\text { Population living in households with an } \\
\text { improved drinking-water source_NFHS } 5\end{array}$} \\
\hline & \begin{tabular}{l|l} 
NFHS 4 \\
\end{tabular} & NFHS 5 & CHANGE \\
\hline INDIA & 94.4 & 95.9 & 1.5 \\
\hline Puducherry & 99.6 & 99.9 & 0.3 \\
\hline NCT Delhi & 99.8 & 99.4 & -0.4 \\
\hline BHAR & 98.4 & 99.2 & 0.8 \\
\hline Ulttar Pradesh & 98.4 & 99.2 & 0.8 \\
\hline Chandigarh & 100.0 & 99.1 & -0.9 \\
\hline Puniab & 99.6 & 98.8 & -0.8 \\
\hline Telangana & 97.7 & 98.7 & 1.0 \\
\hline Haryana & 98.3 & 98.6 & 0.3 \\
\hline Tamil Nadu & 97.7 & 98.6 & 0.9 \\
\hline Goa & 96.7 & 98.5 & 1.8 \\
\hline West bengal & 97.2 & 97.5 & 0.3 \\
\hline Gujjarat & 95.9 & 97.2 & 1.3 \\
\hline Andhra pradesh & 95.6 & 96.7 & 1.1 \\
\hline Rajasthan & 93.7 & 96.5 & 2.8 \\
\hline Andaman \& Nicobar & 95.0 & 96.3 & 1.3 \\
\hline Himachal pradesh & 94.9 & 96.2 & 1.3 \\
\hline Ulttarakhand & 93.6 & 95.9 & 2.3 \\
\hline Mizoram & 93.8 & 95.8 & 2.0 \\
\hline Chattisgarh & 91.3 & 95.5 & 4.2 \\
\hline Dadar\& nagar haveli and Daman \& Dil & na & 95.4 & na \\
\hline \begin{tabular}{|l|} 
Karnataka \\
\end{tabular} & 95.3 & 95.3 & 0.0 \\
\hline Kerala & 94.8 & 94.9 & 0.1 \\
\hline Ârunachal Pradesh & 86.2 & 93.7 & 7.5 \\
\hline Maharastra & 92.5 & 93.5 & 1.0 \\
\hline Lakshadweep & 91.1 & 92.9 & 1.8 \\
\hline Sikkim & 97.8 & 92.8 & -5.0 \\
\hline Jammu and Kashmir & 89.1 & 91.9 & 2.8 \\
\hline Odisha & 89.1 & 91.1 & 2.0 \\
\hline Nagaland & 82.4 & 91.0 & 8.6 \\
\hline Madhya Pradesh & 85.2 & 89.0 & 3.8 \\
\hline \begin{tabular}{|l|} 
Ladakh \\
\end{tabular} & 83.0 & 88.7 & 5.7 \\
\hline Tripura & 86.4 & 880 & 1.6 \\
\hline Jharkhand & 78.1 & 86.6 & 8.5 \\
\hline \begin{tabular}{|l|l|} 
Assamm \\
\end{tabular} & 84.2 & 86.0 & 1.8 \\
\hline Meghalaya & 70.3 & 79.2 & 8.9 \\
\hline Manipur & 63.9 & 77.1 & 13.2 \\
\hline
\end{tabular}

\section{Households with improved sanitation}

\begin{tabular}{|c|c|c|c|}
\hline \multirow{2}{*}{$\begin{array}{l}\text { (States'Uts)|Indicator } \\
\text { ROUND }\end{array}$} & \multicolumn{3}{|c|}{$\begin{array}{l}\text { Population living in } \\
\text { households that use an } \\
\text { improved sanitation } \\
\text { facility_NFHS } 5\end{array}$} \\
\hline & NFHS 4 & \begin{tabular}{|l|} 
NFHS 5 \\
\end{tabular} & CHANGE \\
\hline INDIA & 48.5 & $\begin{array}{l}70.2 \\
\end{array}$ & \begin{tabular}{|l|}
21.7 \\
\end{tabular} \\
\hline Lakshadweep & 99.6 & 99.8 & 0.2 \\
\hline Kerala & 98.2 & 98.7 & 0.5 \\
\hline Mizoram & 84.4 & 95.3 & 10.9 \\
\hline Andaman \& Nicobar & 75.4 & 88.0 & 12.6 \\
\hline Goa & 78.7 & 87.9 & 9.2 \\
\hline Nagaland & 76.7 & 87.7 & 11.0 \\
\hline Sikkim & 89.7 & 87.3 & -2.4 \\
\hline Puniab & 82.7 & 86.6 & 3.9 \\
\hline Chandigarh & \begin{tabular}{|c|}
83.7 \\
\end{tabular} & 85.0 & 1.3 \\
\hline Haryana & 80.6 & 85.0 & 4.4 \\
\hline Puducherry & 64.8 & 84.9 & 20.1 \\
\hline Arunachal Pradesh & 61.6 & 82.9 & 21.3 \\
\hline Meghalaya & 61.4 & 82.9 & 21.5 \\
\hline Himachal pradesh & 72.3 & 81.8 & 9.5 \\
\hline NCT Delhi & 75.1 & 81.1 & 6.0 \\
\hline Utttarakhand & 66.2 & 78.8 & 12.6 \\
\hline Andhra pradesh & 54.4 & 77.3 & 22.9 \\
\hline Chattisgarh & 34.8 & 76.8 & 42.0 \\
\hline Telangana & 52.3 & 76.2 & 23.9 \\
\hline Jammu and Kashmir & 53.8 & 75.7 & 21.9 \\
\hline Karnataka & 57.8 & 74.8 & 17.0 \\
\hline Gujarat & 63.6 & 74.0 & 10.4 \\
\hline Tripura & 63.7 & 73.6 & 9.9 \\
\hline Tamil Nadu & 52.5 & 72.6 & 20.1 \\
\hline Maharastra & 52.3 & 72.0 & 19.7 \\
\hline Rajasthan & 46.1 & 71.1 & 25.0 \\
\hline Uttar Pradesh & 36.4 & 68.8 & 32.4 \\
\hline Assam & 49.0 & 68.6 & 19.6 \\
\hline West bengal & 52.8 & 68.0 & 15.2 \\
\hline Dadar\& nagar haveli and Daman \& Dit & 44.4 & 65.8 & 21.4 \\
\hline Madhya Pradesh & 34.8 & 65.1 & 30.3 \\
\hline Manipur & 52.6 & 64.9 & 12.3 \\
\hline Ddisha & 30.0 & 60.5 & 30.5 \\
\hline Jharkhand & 25.0 & 56.7 & 31.7 \\
\hline $\mathrm{BH} A \mathrm{~A}$ & 26.5 & 49.4 & 22.9 \\
\hline Ladakh & 17.8 & 42.3 & 24.5 \\
\hline
\end{tabular}




\section{Household \& population profile-Gender aspects}

mprovement in the indicator

Decline in the indicator

No change

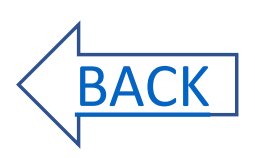

\begin{tabular}{|c|c|c|c|}
\hline \multicolumn{4}{|c|}{ Sex ratio } \\
\hline \multirow{2}{*}{$\begin{array}{l}\text { (StatelUts)]Indicators } \\
\text { Fiound }\end{array}$} & \multicolumn{3}{|c|}{$\begin{array}{l}\text { Sex ratio of the total } \\
\text { population (females per } 1000 \\
\text { males)_NFHS } 5\end{array}$} \\
\hline & NFHS4 & NFHS5 & Change \\
\hline NDIA & 991 & 1020 & 29 \\
\hline akshadweep & 1022 & 1187 & 165 \\
\hline Kerala & 1049 & 1121 & 72 \\
\hline Puducherry & 1068 & 1112 & 44 \\
\hline Bihar & 1062 & 1090 & 28 \\
\hline Tamil Nadu & 1033 & 1088 & 55 \\
\hline Manipur & 1049 & 1066 & 17 \\
\hline Ddisha & 1036 & 1063 & 27 \\
\hline Jharkhand & 1002 & 1050 & 48 \\
\hline Telangana & 1007 & 1049 & 42 \\
\hline West bengal & 1011 & 1049 & 38 \\
\hline endhra pradesh & 1021 & 1045 & 24 \\
\hline Himachal pradesh & 1078 & 1040 & -38 \\
\hline Meghalaya & 1005 & 1039 & 34 \\
\hline Karnataka & 979 & 1034 & 55 \\
\hline Foa & 1018 & 1027 & 9 \\
\hline Mizoram & 1012 & 1018 & 6 \\
\hline Iltar Pradesh & 995 & 1017 & 22 \\
\hline Ittarakhand & 1015 & 1016 & 1 \\
\hline Chattisgarh & 1019 & 1015 & -4 \\
\hline 4 ssam & 993 & 1012 & 19 \\
\hline Tripura & 998 & 1011 & 13 \\
\hline Baiasthan & 973 & 1009 & 36 \\
\hline Nagaland & 968 & 1007 & 39 \\
\hline Arunachal Pradesh & 958 & 997 & 39 \\
\hline iikkim & 942 & 990 & 48 \\
\hline adakh & 1000 & 971 & -29 \\
\hline Madhya Pradesh & 948 & 970 & 22 \\
\hline Maharastra & 952 & 966 & 14 \\
\hline Gujarat & 950 & 965 & 15 \\
\hline Andaman \& Nicobar & 977 & 963 & -14 \\
\hline Jammu and Kashmir & 971 & 948 & -23 \\
\hline Punjab & 905 & 938 & 33 \\
\hline Haryana & 876 & 926 & 50 \\
\hline Chandigarh & 934 & 917 & -17 \\
\hline NCT Delhi & 854 & 913 & 59 \\
\hline Padar\& nagar haveli and Daman \& Diu & 813 & 827 & 14 \\
\hline
\end{tabular}

Women with 10 or more years of schooling

\begin{tabular}{|c|c|c|c|}
\hline (State/Uts)/Indicators & $\begin{array}{l}\text { Wome } \\
\text { years of }\end{array}$ & $\begin{array}{l}\text { n with } 100 \\
\text { schooling }\end{array}$ & $\begin{array}{l}\text { or more } \\
\text { NFHS } 5\end{array}$ \\
\hline Round & NFHS4 & NFHS5 & Change \\
\hline INDIA & 35.7 & 41.0 & 5.3 \\
\hline Kerala & 72.2 & 77.0 & 4.8 \\
\hline Goa & 58.2 & 71.5 & 13.3 \\
\hline Lakshadweep & 56.8 & 67.8 & 11.0 \\
\hline Himachal pradesh & 59.4 & 65.9 & 6.5 \\
\hline Puducherry & 60.3 & 65.4 & 5.1 \\
\hline NCT Delhi & 54.2 & 59.7 & 5.5 \\
\hline Chandigarh & 59.2 & 59.6 & 0.4 \\
\hline Tamil Nadu & 50.9 & 56.6 & 5.7 \\
\hline Punjab & 55.1 & 56.0 & 0.9 \\
\hline Andaman \& Nicobar & 49.1 & 52.5 & 3.4 \\
\hline Jammu and Kashmir & 37.1 & 51.3 & 14.2 \\
\hline Maharastra & 42.0 & 50.4 & 8.4 \\
\hline Uttarakhand & 44.6 & 50.4 & 5.8 \\
\hline Karnataka & 45.5 & 50.2 & 4.7 \\
\hline Mizoram & 39.9 & 50.0 & 10.1 \\
\hline Ladakh & 44.6 & 50.0 & 5.4 \\
\hline Haryana & 45.8 & 49.5 & 3.7 \\
\hline Sikkim & 40.7 & 49.0 & 8.3 \\
\hline Manipur & 45.9 & 48.1 & 2.2 \\
\hline Telangana & 43.6 & 45.5 & 1.9 \\
\hline Nagaland & 33.3 & 44.4 & 11.1 \\
\hline Andhra pradesh & 34.3 & 39.6 & 5.3 \\
\hline Arunachal Pradesh & 31.0 & 39.4 & 8.4 \\
\hline Uttar Pradesh & 32.9 & 39.3 & 6.4 \\
\hline Chattisgarh & 26.5 & 36.9 & 10.4 \\
\hline Dadar\& nagar haveli and Daman \& Di & 40.3 & 35.8 & -4.5 \\
\hline Meghalaya & 33.6 & 35.1 & 1.5 \\
\hline Gujarat & 33.0 & 33.8 & 0.8 \\
\hline Rajasthan & 25.1 & 33.4 & 8.3 \\
\hline Jharkhand & 28.7 & 33.2 & 4.5 \\
\hline Odisha & 26.7 & 33.0 & 6.3 \\
\hline West bengal & 26.5 & 32.9 & 6.4 \\
\hline Assam & 26.2 & 29.6 & 3.4 \\
\hline Madhya Pradesh & 23.2 & 29.3 & 6.1 \\
\hline Bihar & 22.8 & 28.8 & 6.0 \\
\hline Tripura & 23.4 & 23.2 & -0.2 \\
\hline
\end{tabular}

States arranged as per highest to lowest Prevalence (NFHS-5)
Women age $\mathbf{2 0 - 2 4}$ years married before 18 years

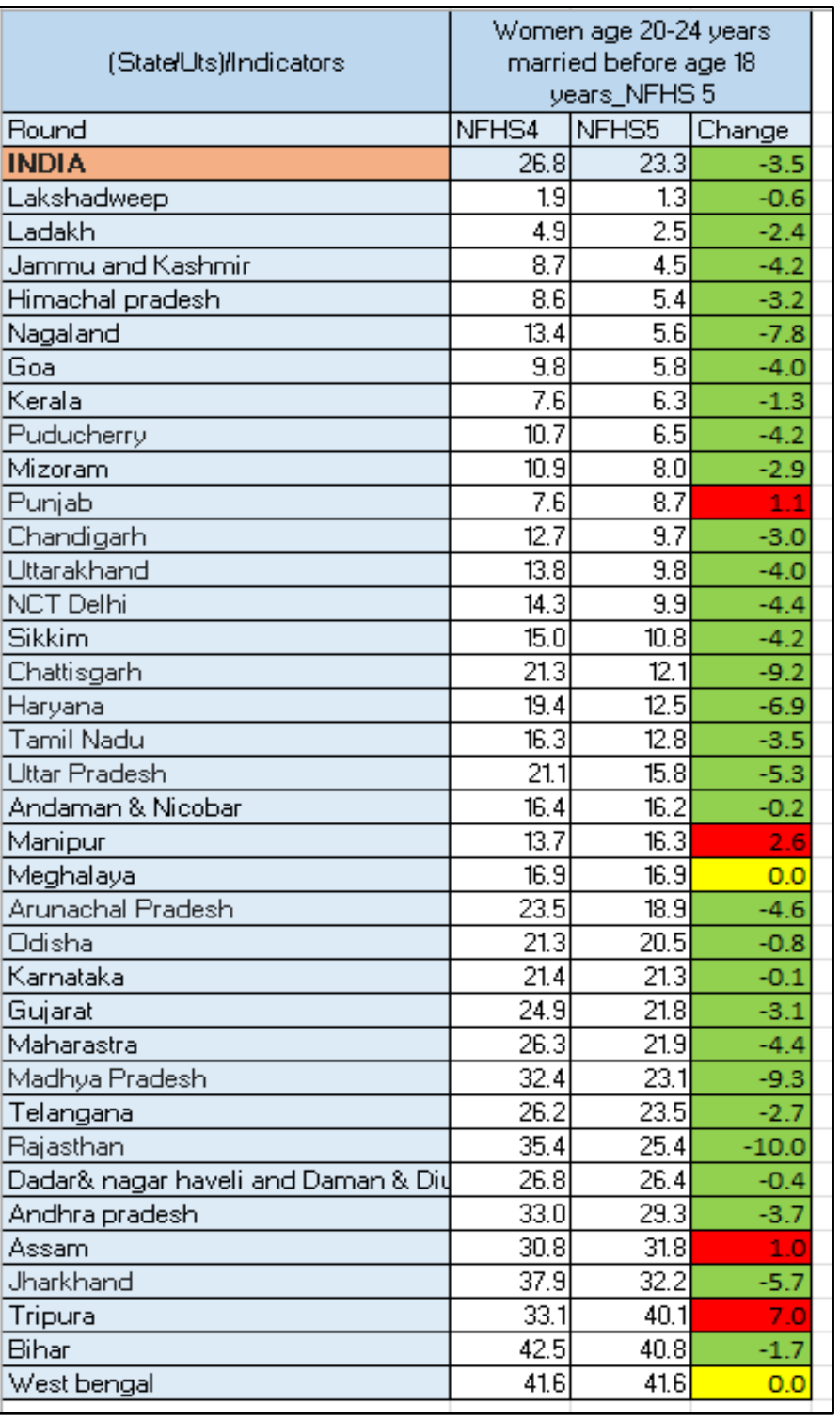

States arranged as per lowest to highest prevalence (NFHS-5) 
Hypertension among women and men

Women

States arranged as per lowest to highest prevalence (NFHS-5)

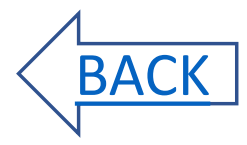

\begin{tabular}{|c|c|c|}
\hline (StatelUts)ilndicators & $\begin{array}{r}\text { Women Eleve } \\
\text { pressure (Sys } \\
\mathrm{mm} \text { of } \mathrm{Hg} \\
\text { Diastolic } \geq 90 \\
\text { or taking } \mathrm{me} \\
\text { control blooc }\end{array}$ & $\begin{array}{l}\text { ated blood } \\
\text { stolic } \geq 140 \\
\text { and/or } \\
\mathrm{mm} \text { of } \mathrm{Hg} \text { ) } \\
\text { edicine to } \\
\text { d pressure }\end{array}$ \\
\hline Round & \begin{tabular}{|l|l|}
$\mathrm{NFHS}-4$ \\
\end{tabular} & NFHS-5 \\
\hline INDIA & na & 21.3 \\
\hline Dadra \& Nagar Haveli And Daman \& Diu & na & 14.9 \\
\hline Rajasthan & na & 15.4 \\
\hline Ladakh & na & 15.7 \\
\hline Bihar & na & 15.9 \\
\hline Mizoram & na & 17.7 \\
\hline Jharkhand & na & 17.8 \\
\hline Uttar Pradesh & na & 18.4 \\
\hline Meghalaya & na & 18.7 \\
\hline Assam & na & 19.1 \\
\hline Jammu \& Kashmir & na & 20.0 \\
\hline West Bengal & na & 20.5 \\
\hline Gujarat & na & 20.6 \\
\hline Madhya Pradesh & na & 20.6 \\
\hline Tripura & na & 20.9 \\
\hline Haryana & na & 21.0 \\
\hline Himachal Pradesh & na & 22.2 \\
\hline Nagaland & na & 22.4 \\
\hline Ddisha & na & 22.4 \\
\hline Uttarakhand & na & 22.9 \\
\hline Puducherry & na & 23.0 \\
\hline Manipur & na & 23.0 \\
\hline Maharashtra & na & 23.1 \\
\hline Chhattisgarh & na & 23.6 \\
\hline Net Df Delhi & na & 24.1 \\
\hline Tamilnadu & na & 24.8 \\
\hline Lakshadweep & na & 24.8 \\
\hline Arunachal Pradesh & na & 24.9 \\
\hline Karnataka & na & 25.0 \\
\hline Chandigarh & na & 25.0 \\
\hline Andhra Pradesh & na & 25.3 \\
\hline Andaman \& Nicobar Islands & na & 25.3 \\
\hline Telangana & na & 26.1 \\
\hline Goa & na & 27.5 \\
\hline Kerala & na & 30.9 \\
\hline Punjab & na & 31.2 \\
\hline Sikkim & na & 34.5 \\
\hline
\end{tabular}

Men Elevated blood pressure [Systolic $\geq 14$ $\mathrm{mm}$ of $\mathrm{Hg}$ and

$\geq 90 \mathrm{~mm}$ of $\mathrm{Hgl}$ or taking medicine to control blood Pressure [\%]

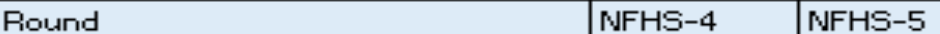
NFHS-5

Dadra \&agar HaveliAnd Daman Bur Ladakh

Rajasthan

Bihar

Jammu \& Kashmir

West Benga

Assam

Meghalaya

Uttar Pradesh

Jharkhand

Madhya Pradesh

Tripura

Himachal Pradesh

Maharashtra

Lakshadweep

Haryana

Mizoram

Goa

Karnataka

Chhattisgarh

Nagaland

Andhra Pradesh

Tamilnadu

Andaman \& Nicobar Islands

Chandigarh

Telangana

Ner Df Delhi

Kerala

Arunachal Pradesh

Manipur

Puniab

Sikkim

\begin{tabular}{|c|c|}
\hline & \\
\hline 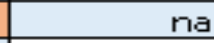 & 24.0 \\
\hline na & 15.4 \\
\hline na & 17.4 \\
\hline na & 17.9 \\
\hline na & 18.4 \\
\hline na & 18.9 \\
\hline na & 20.1 \\
\hline na & 20.3 \\
\hline na & 20.3 \\
\hline na & 21.4 \\
\hline na & 21.7 \\
\hline na & 22.6 \\
\hline na & 22.7 \\
\hline na & 22.7 \\
\hline na & 24.4 \\
\hline na & 24.4 \\
\hline na & 24.7 \\
\hline na & 25.1 \\
\hline na & 25.2 \\
\hline na & 25.6 \\
\hline na & 26.8 \\
\hline na & 26.9 \\
\hline na & 27.7 \\
\hline na & 28.7 \\
\hline na & 29.0 \\
\hline na & 30.1 \\
\hline na & 30.2 \\
\hline na & 30.2 \\
\hline na & 30.6 \\
\hline na & 31.4 \\
\hline na & 31.8 \\
\hline na & 32.8 \\
\hline na & 32.8 \\
\hline na & 33.1 \\
\hline na & 33.2 \\
\hline na & 37.7 \\
\hline na & 41.6 \\
\hline
\end{tabular}




\section{Change in percent point graphs}

NOTE: States/UTs missing in the graphs indicates that their data is not available 
Neonatal Mortality Rate (NNMR)

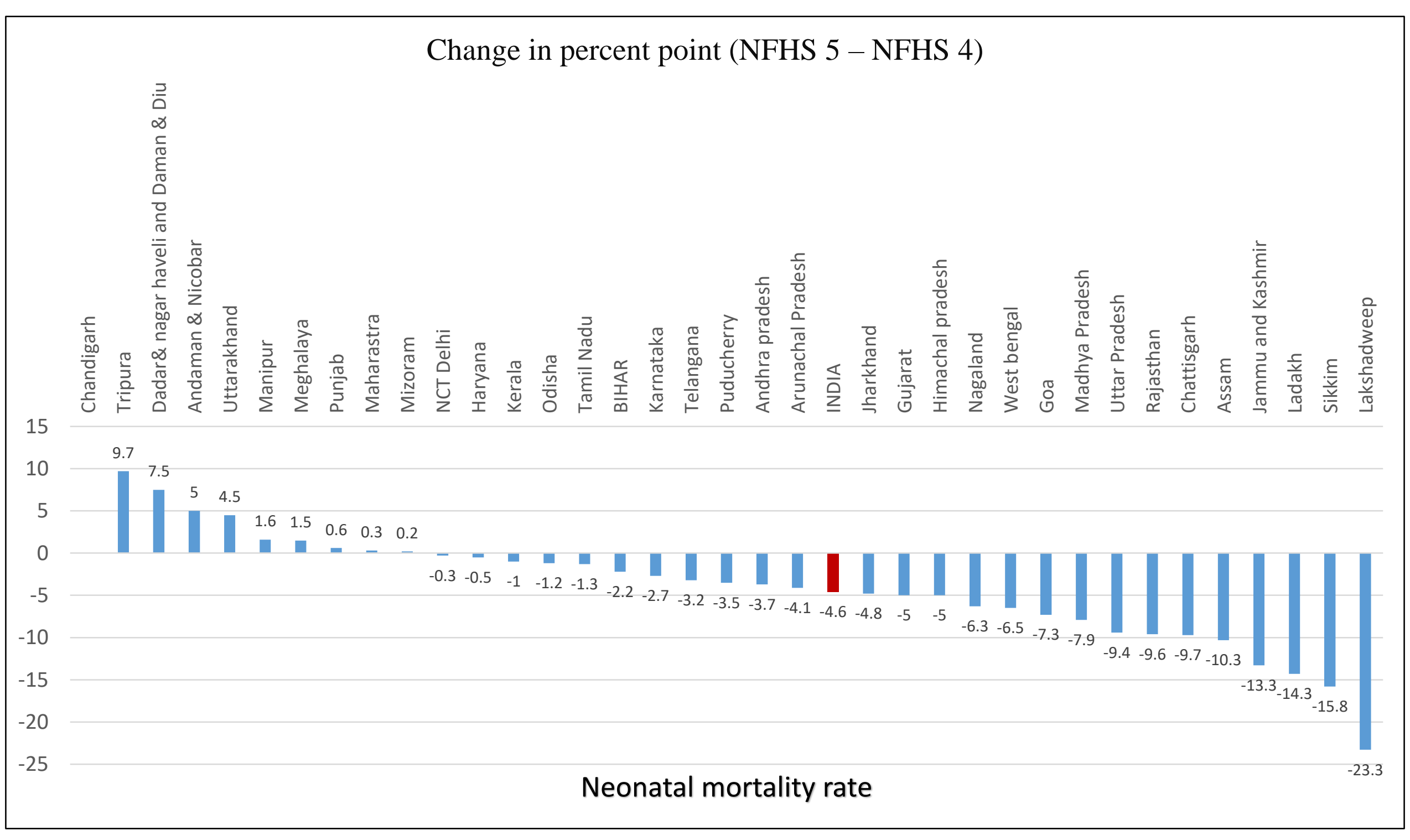




\section{Infant Mortality Rate (IMR)}

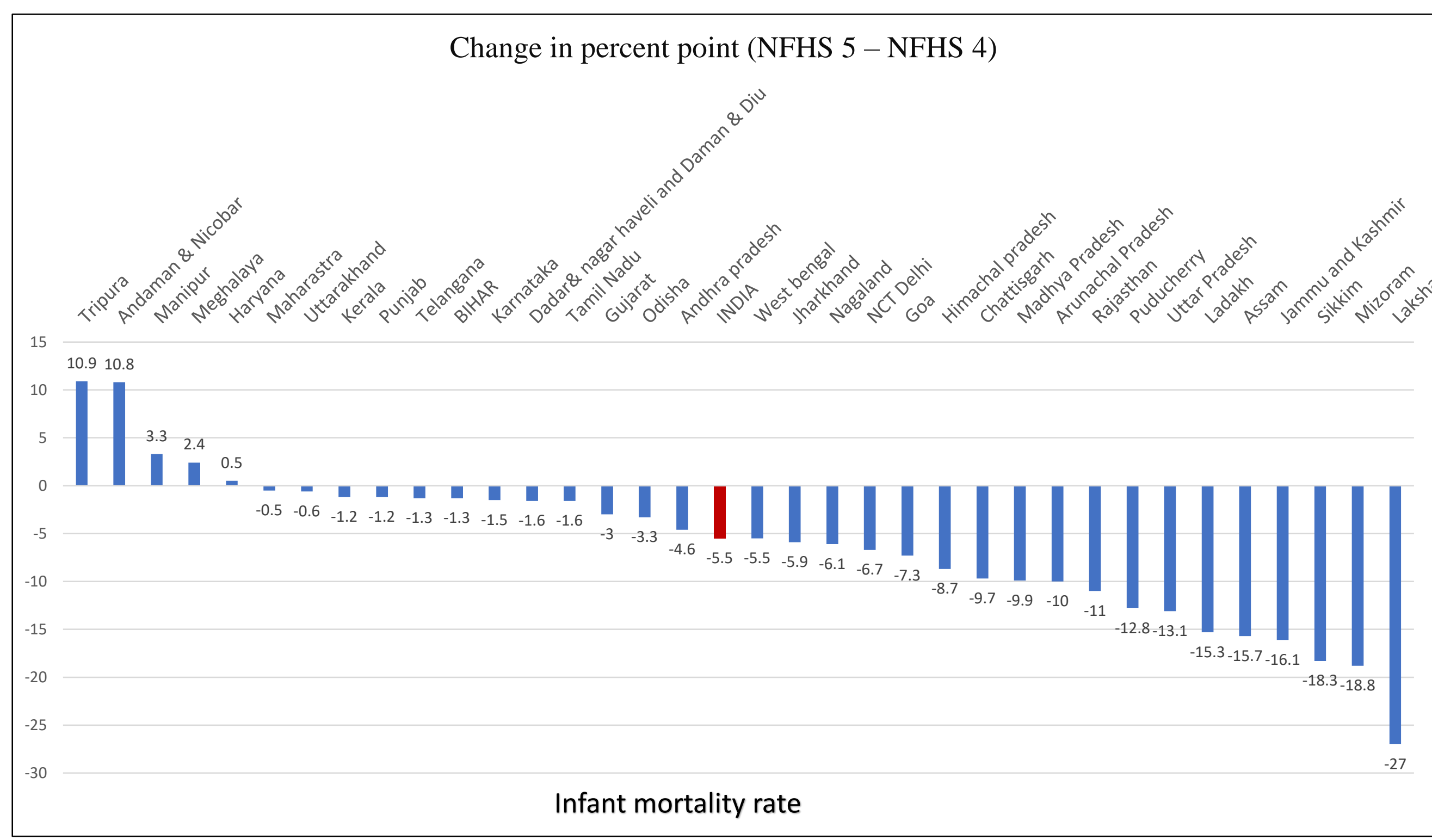




\section{Under-five Mortality Rate (U5MR)}

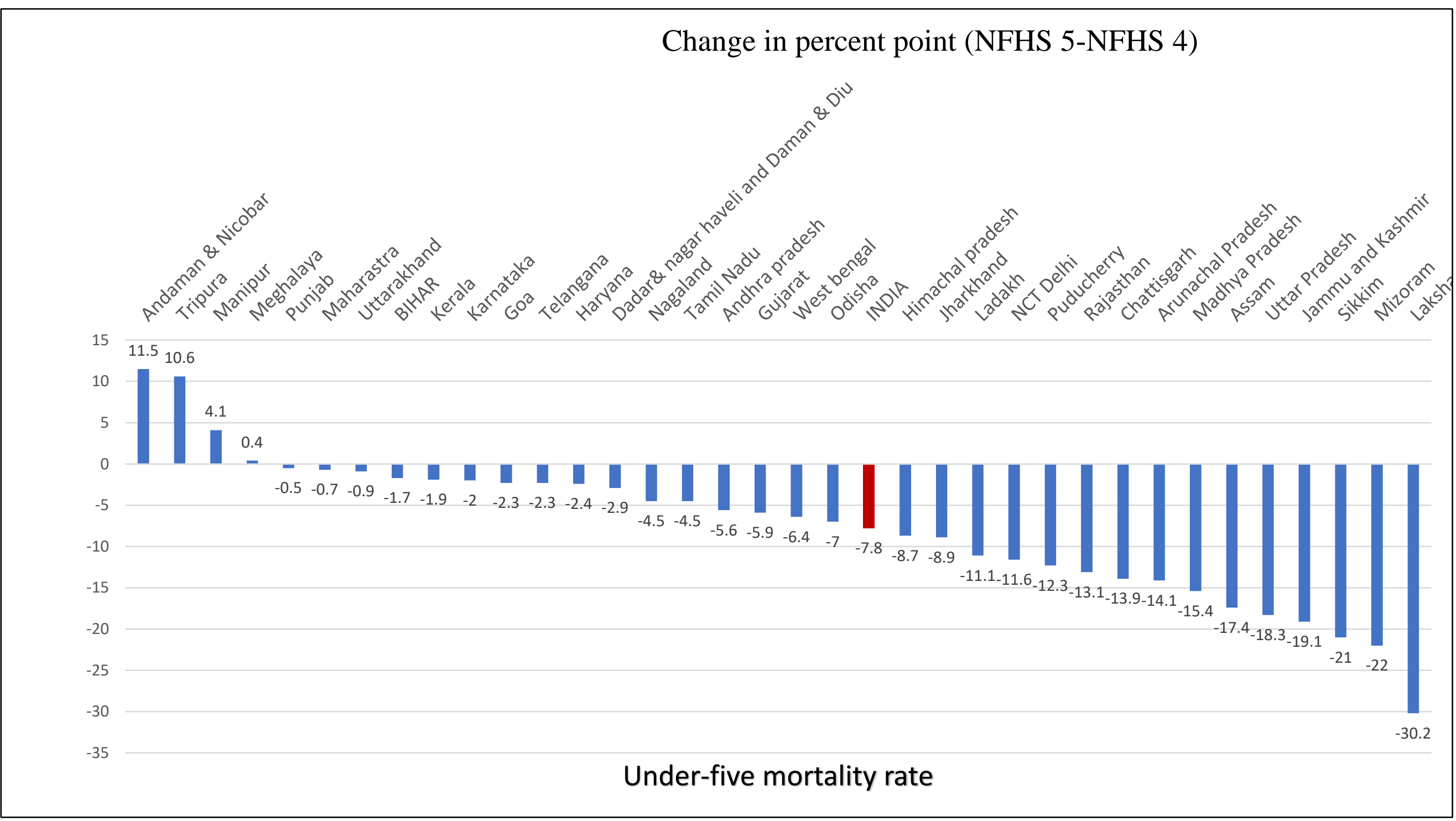




\section{Children under 5 years who are stunted}

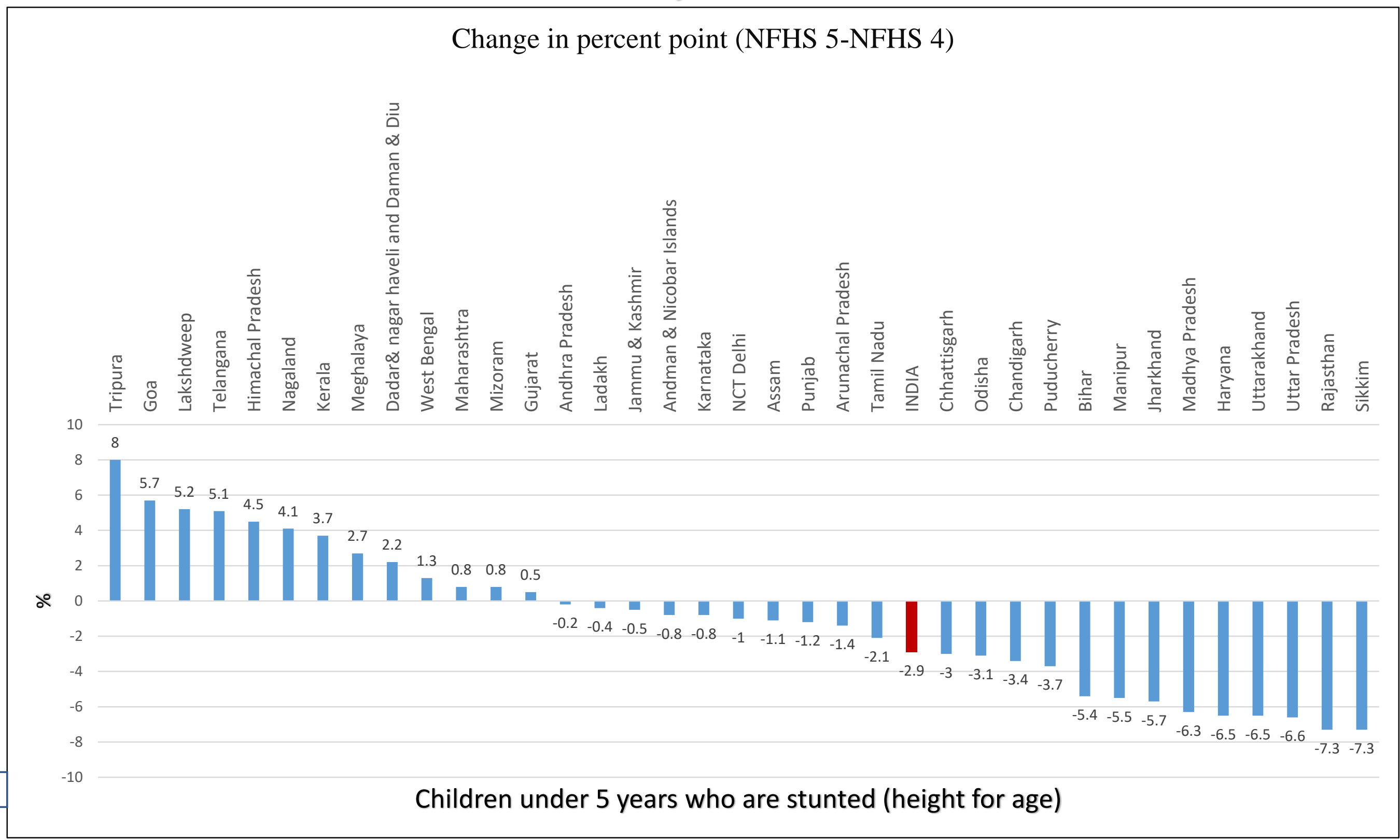


Children under 5 years who are wasted

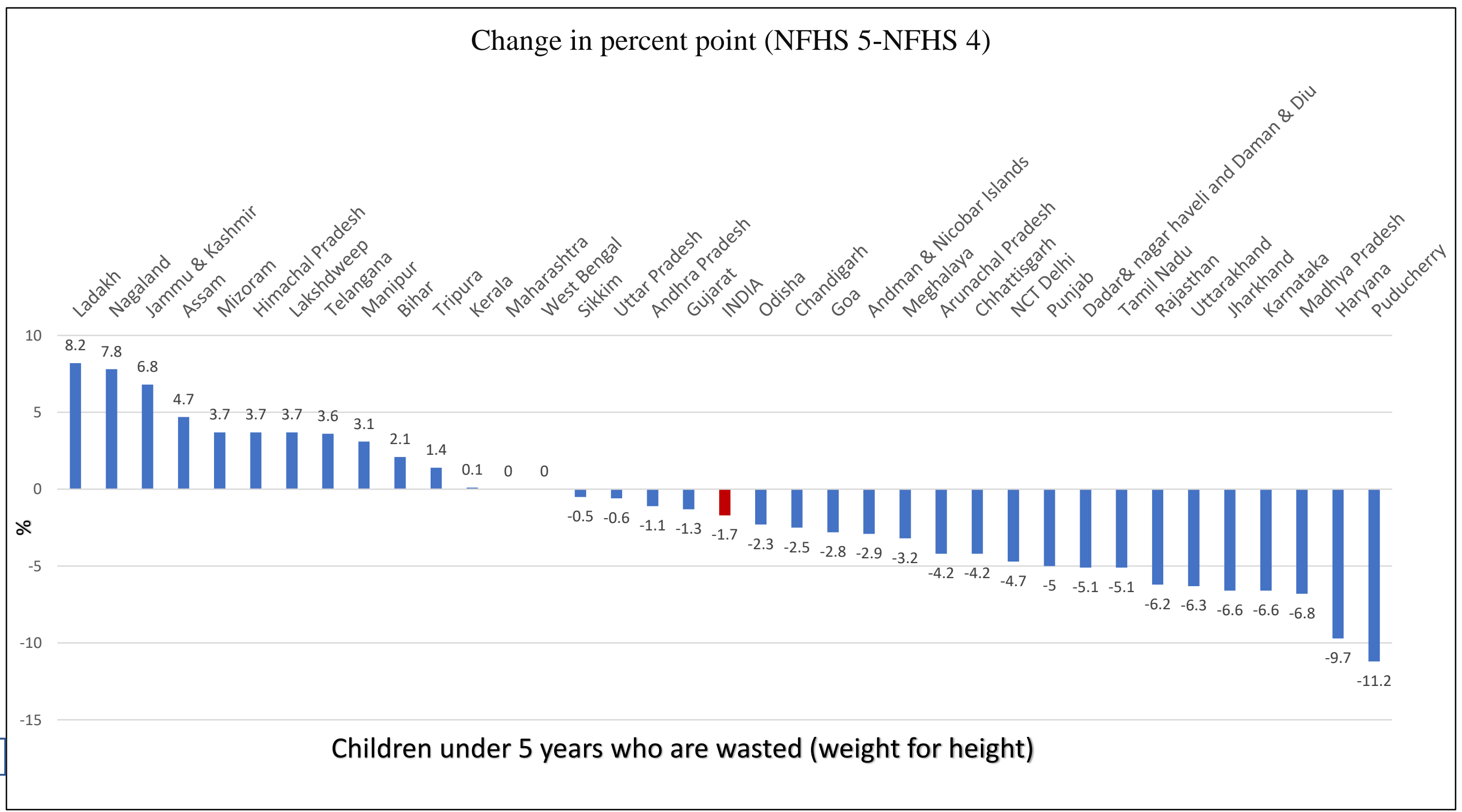




\section{Children under 5 years who are severely wasted}

Change in percent point (NFHS 5-NFHS 4)

8.0

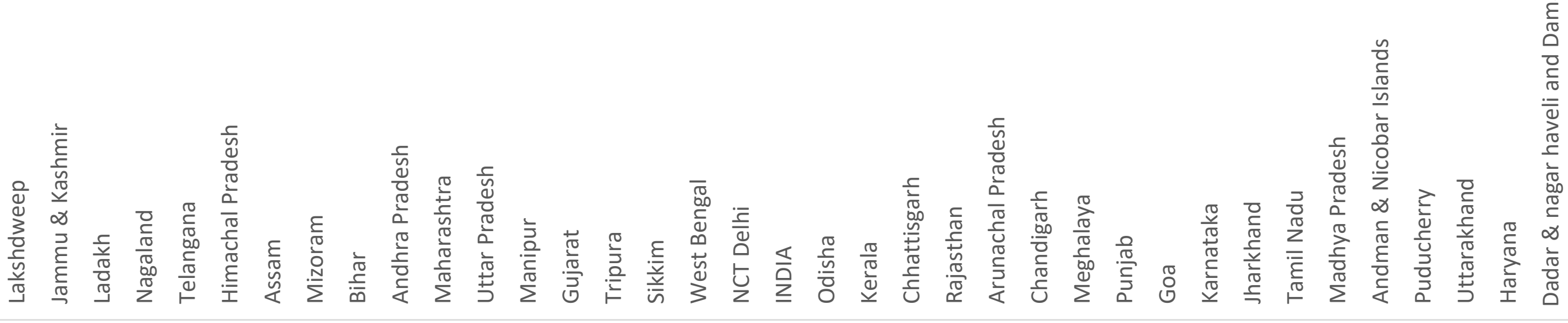

6.0 5.8 


\section{Children under 5 years who are underweight}

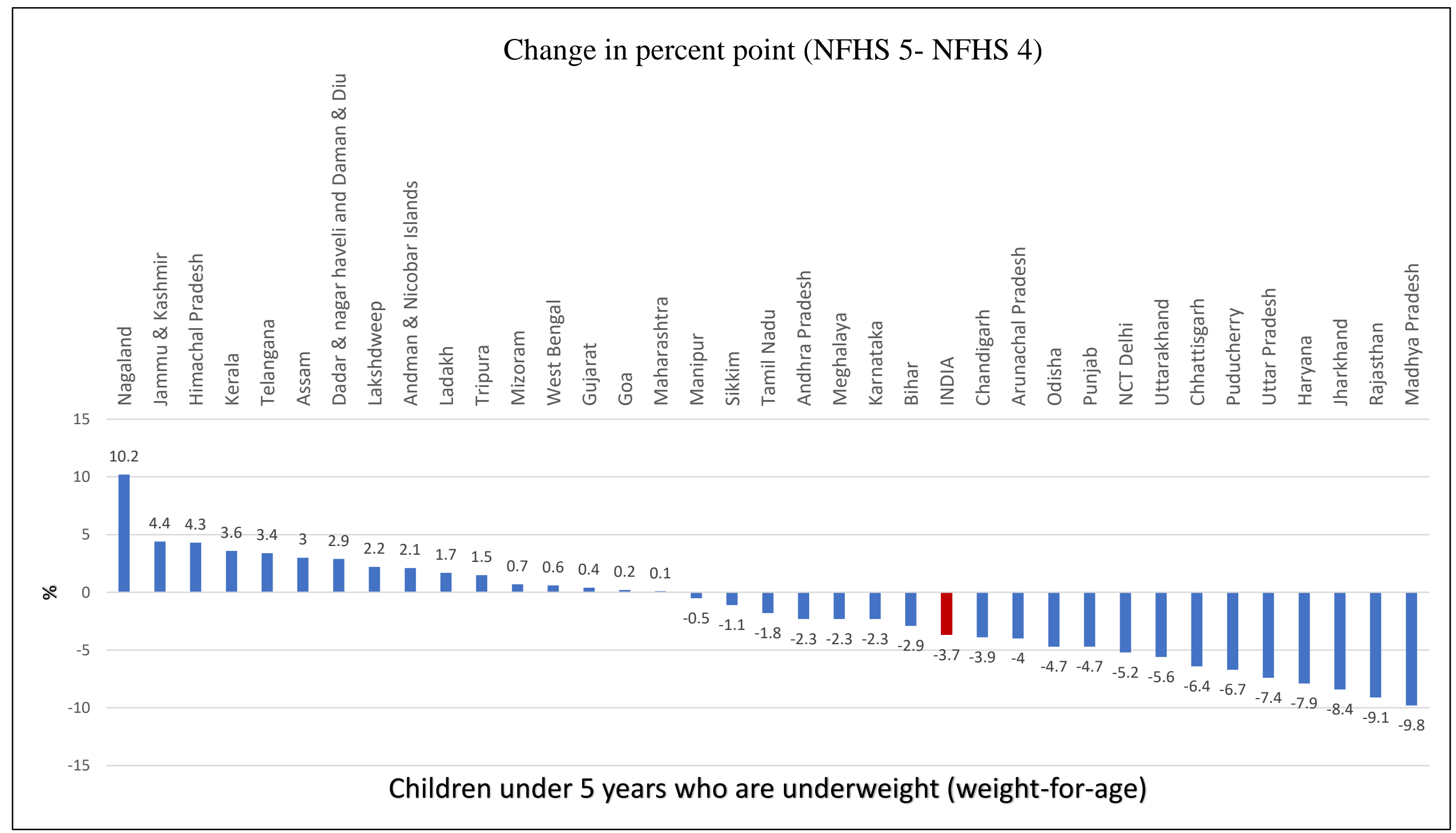




\section{Children under 5 years who are Overweight}

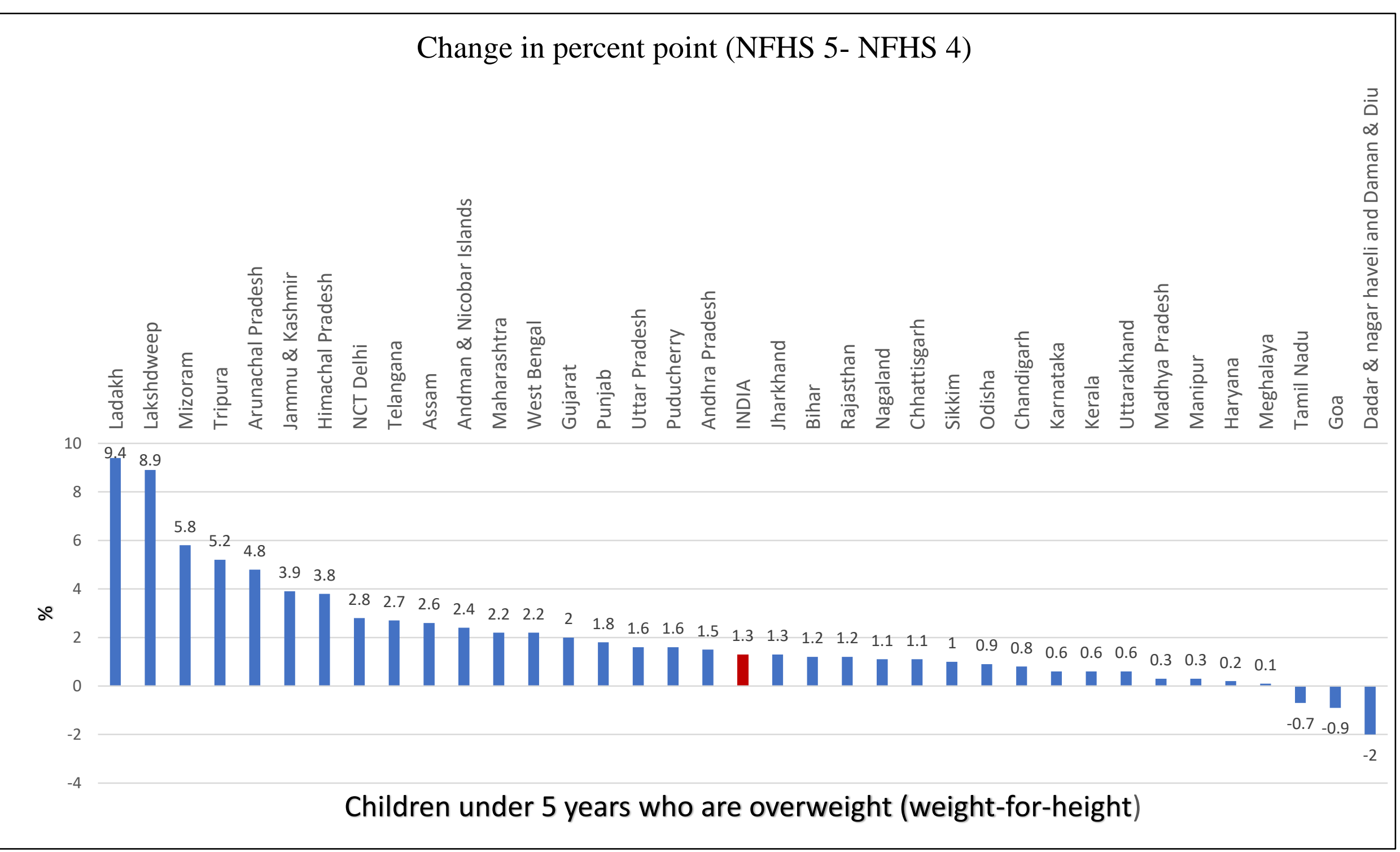




\section{Women Underweight}

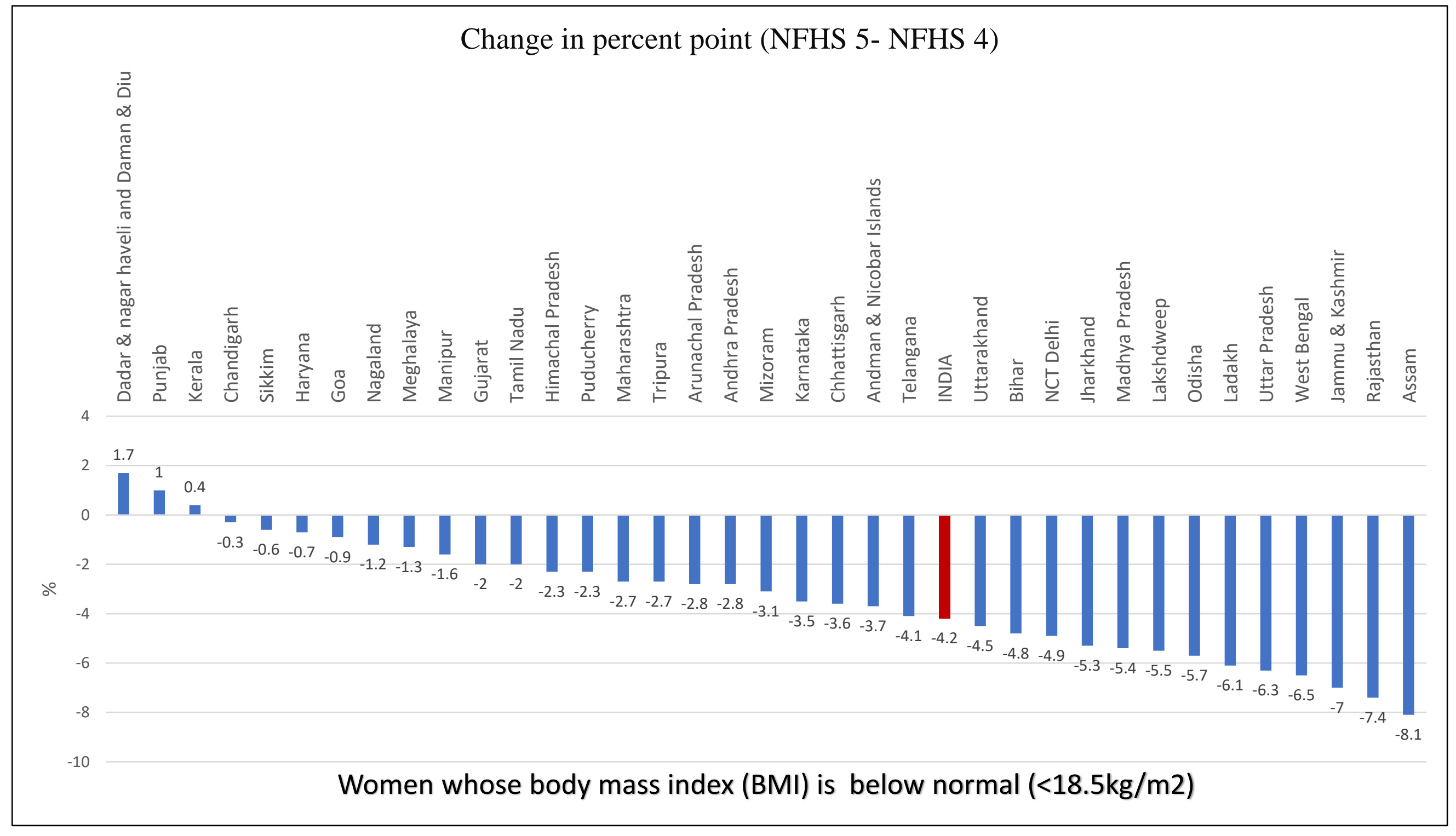




\section{Women Overweight}

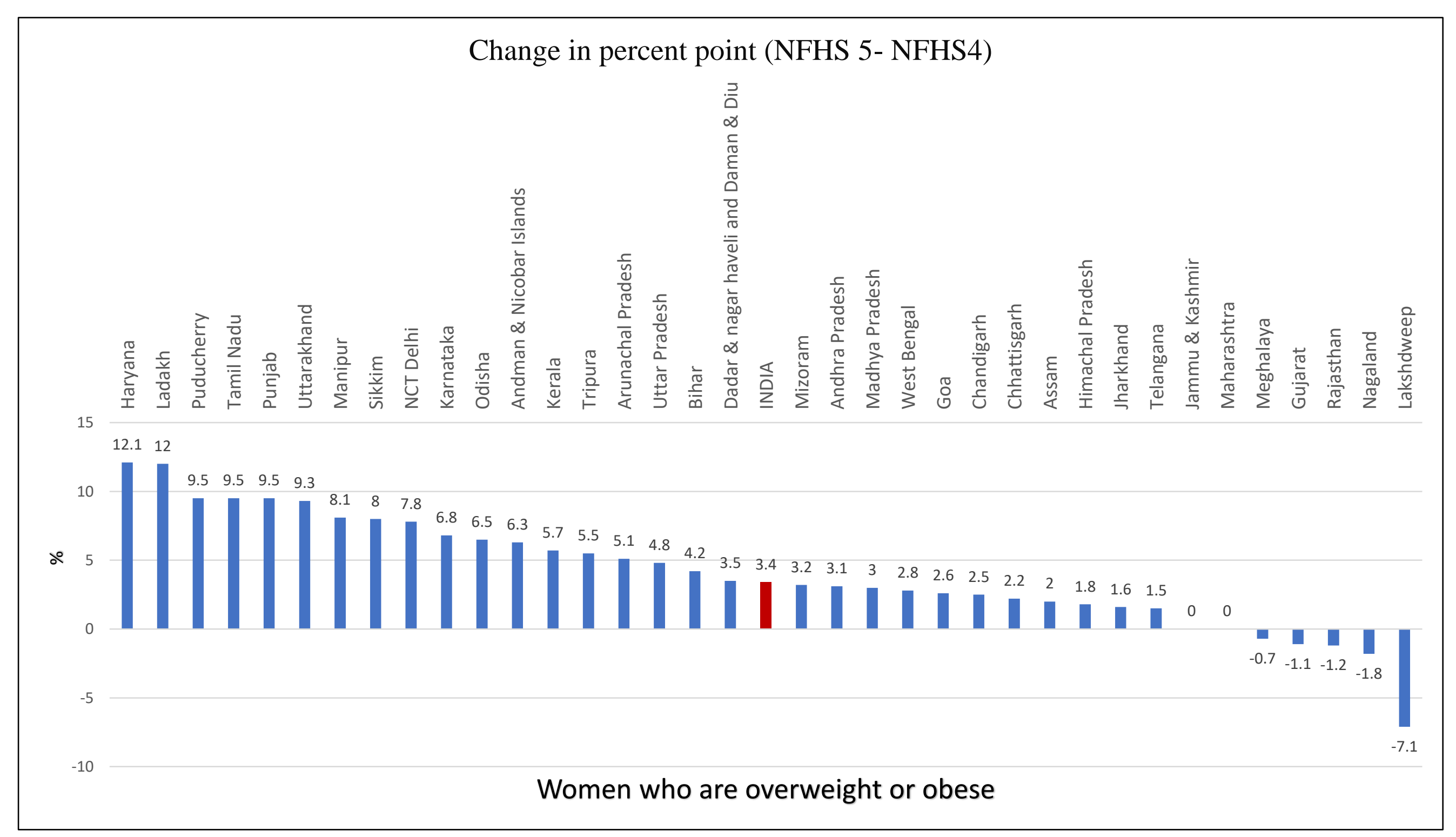




\section{Men Underweight}

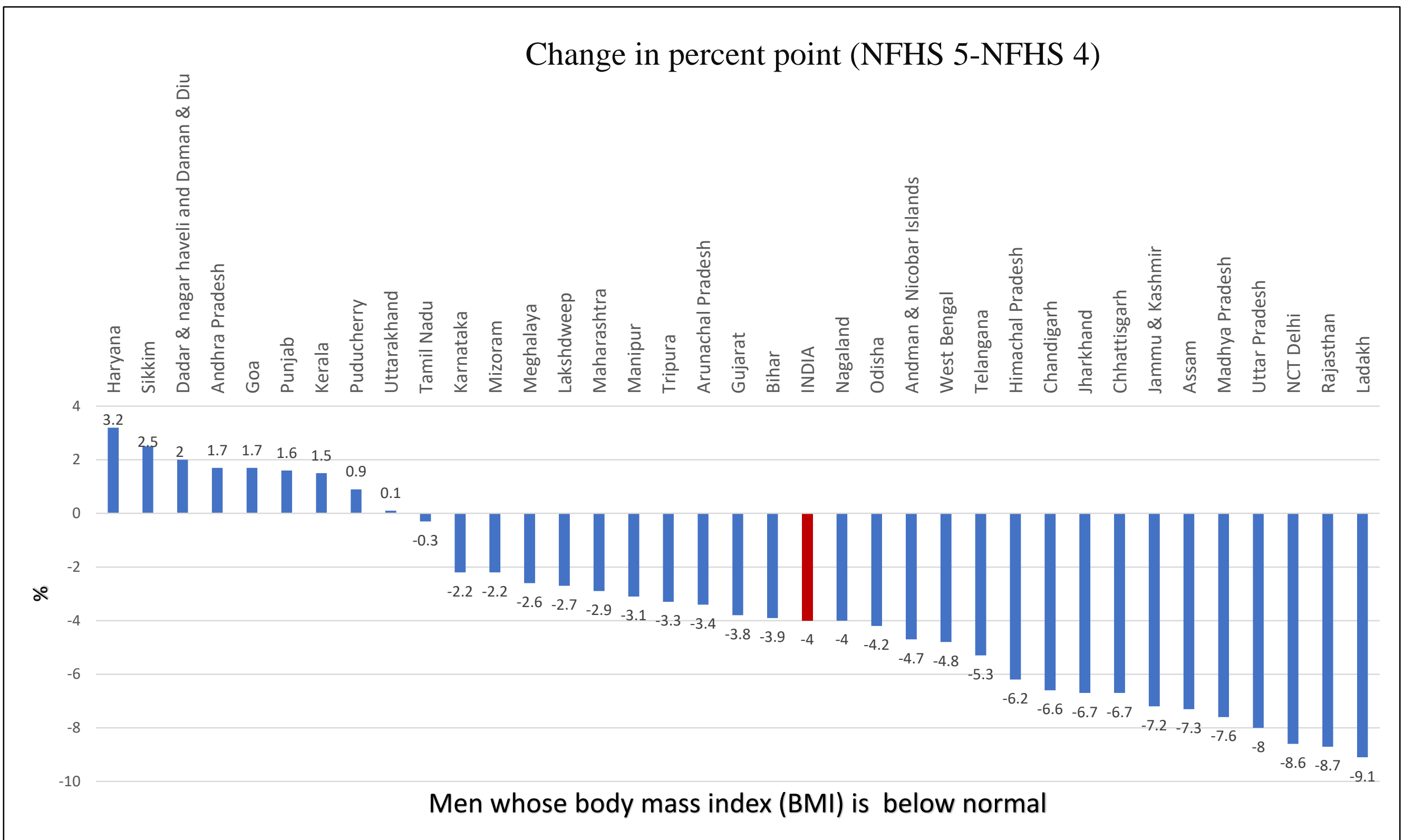




\section{Men Overweight}

Change in percent point (NFHS 5- NFHS 4) 
Children aged 6-59 months who are anemic

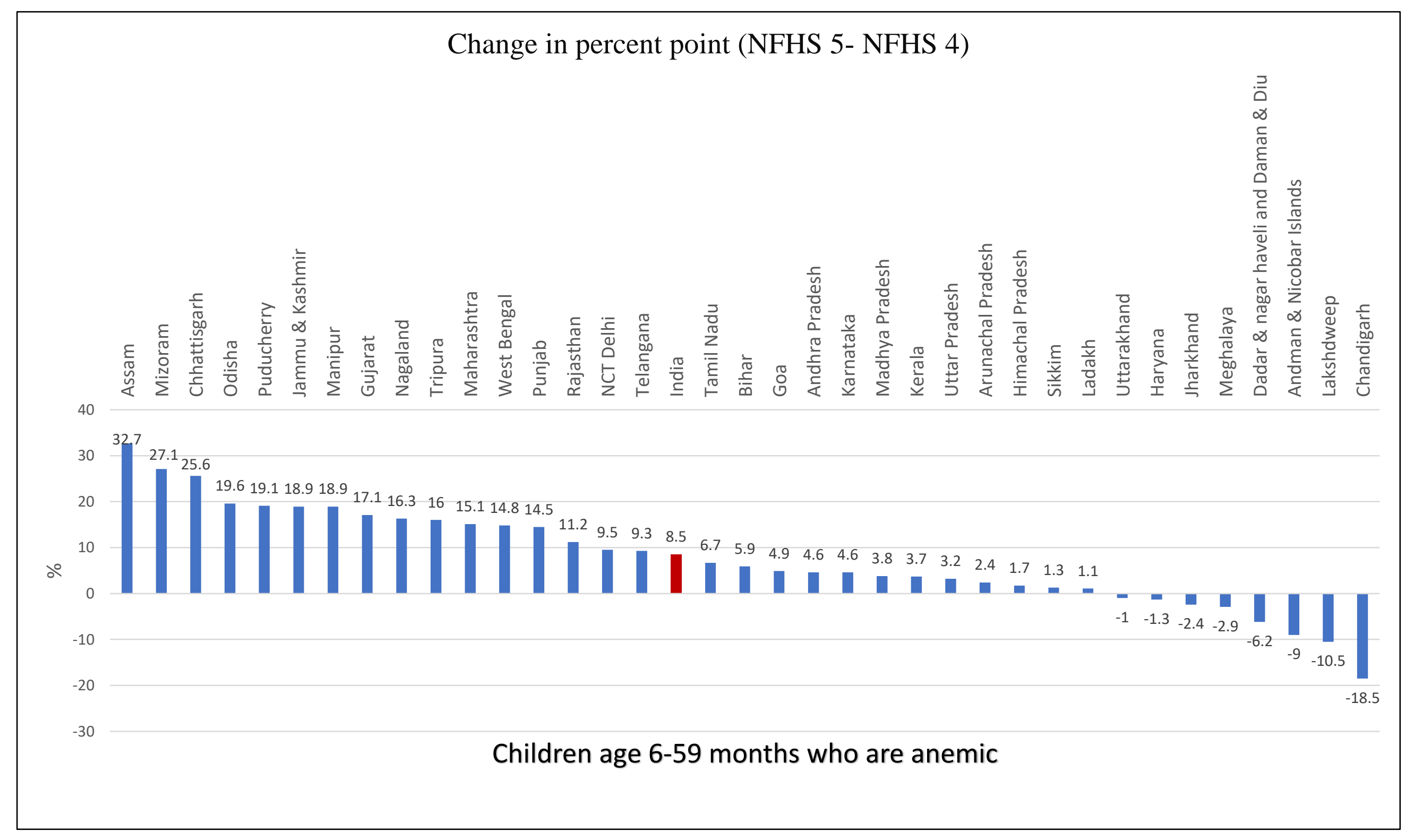


Adolescent girls who are anemic

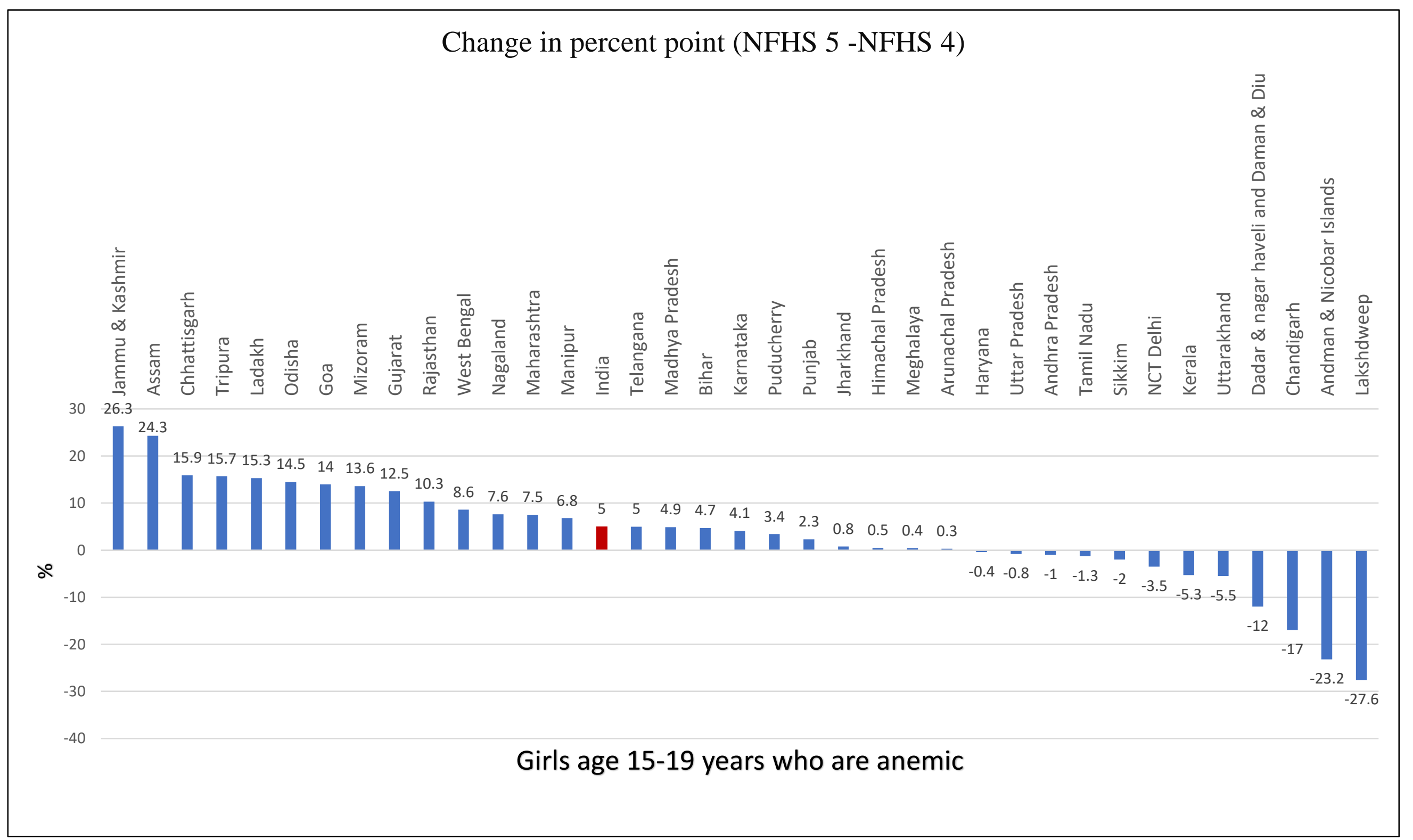


Adolescent boys who are anemic

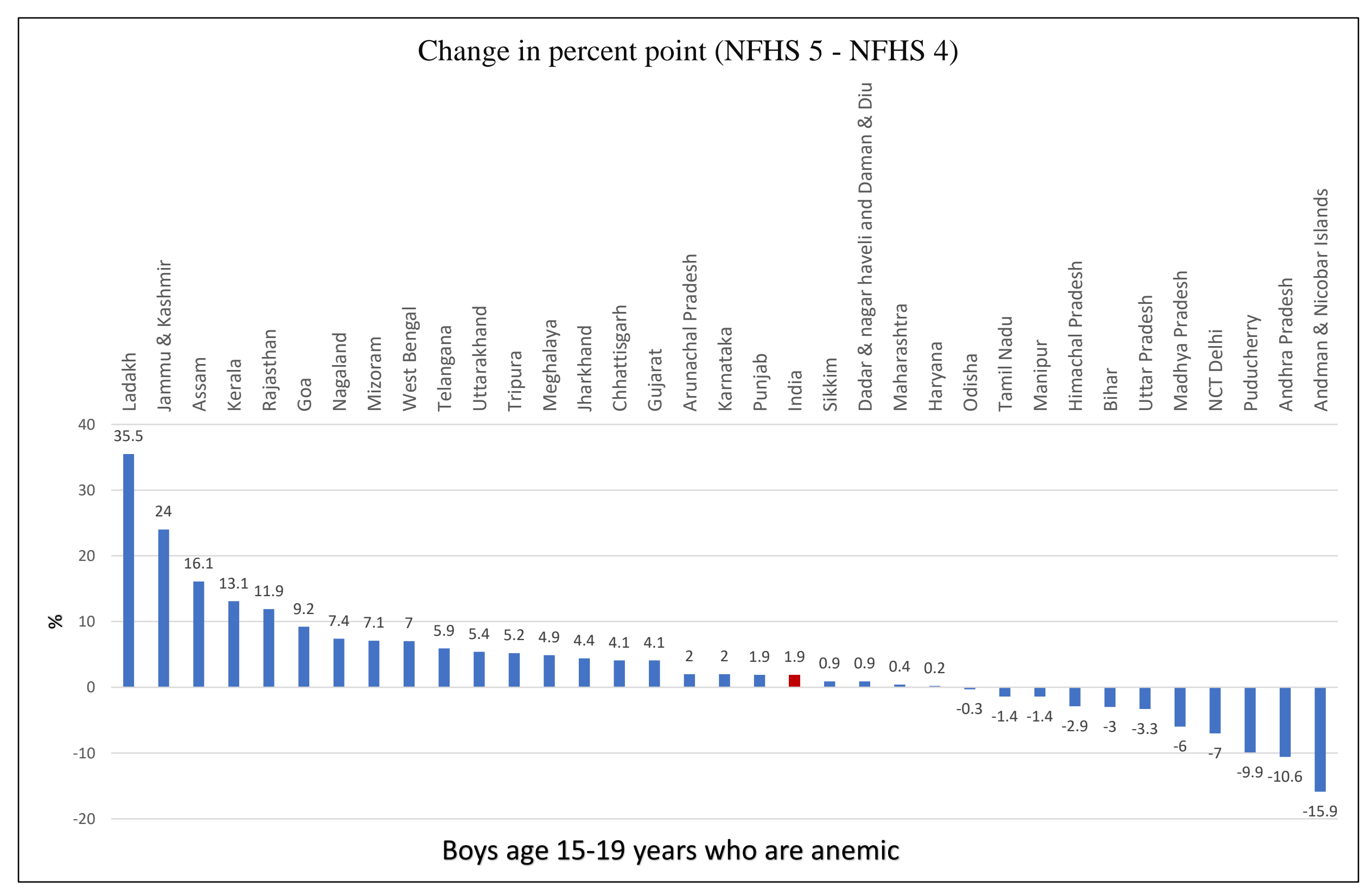


Pregnant women aged 15-49 years who are anemic

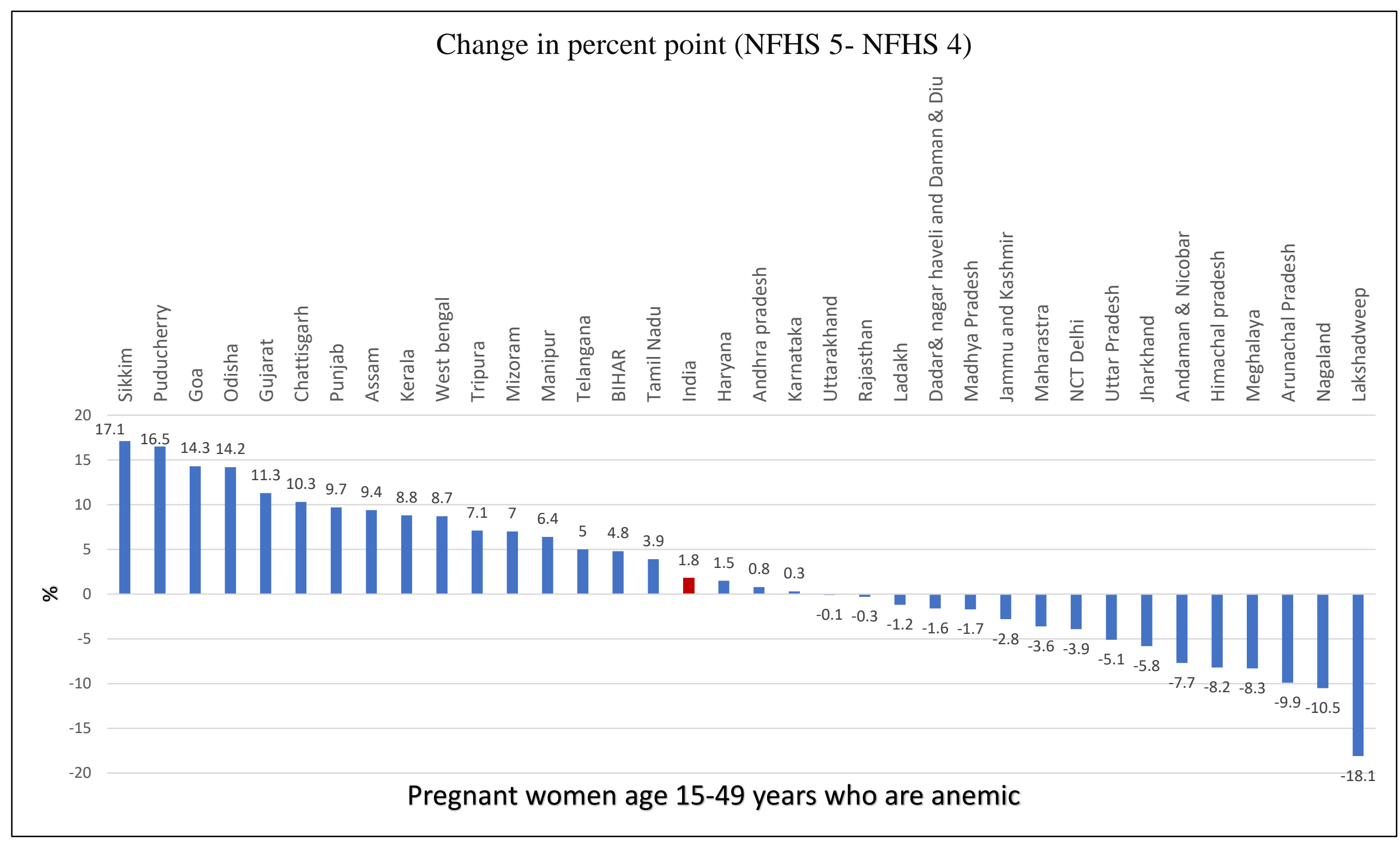




\section{All women aged 15-49 years who are anemic}

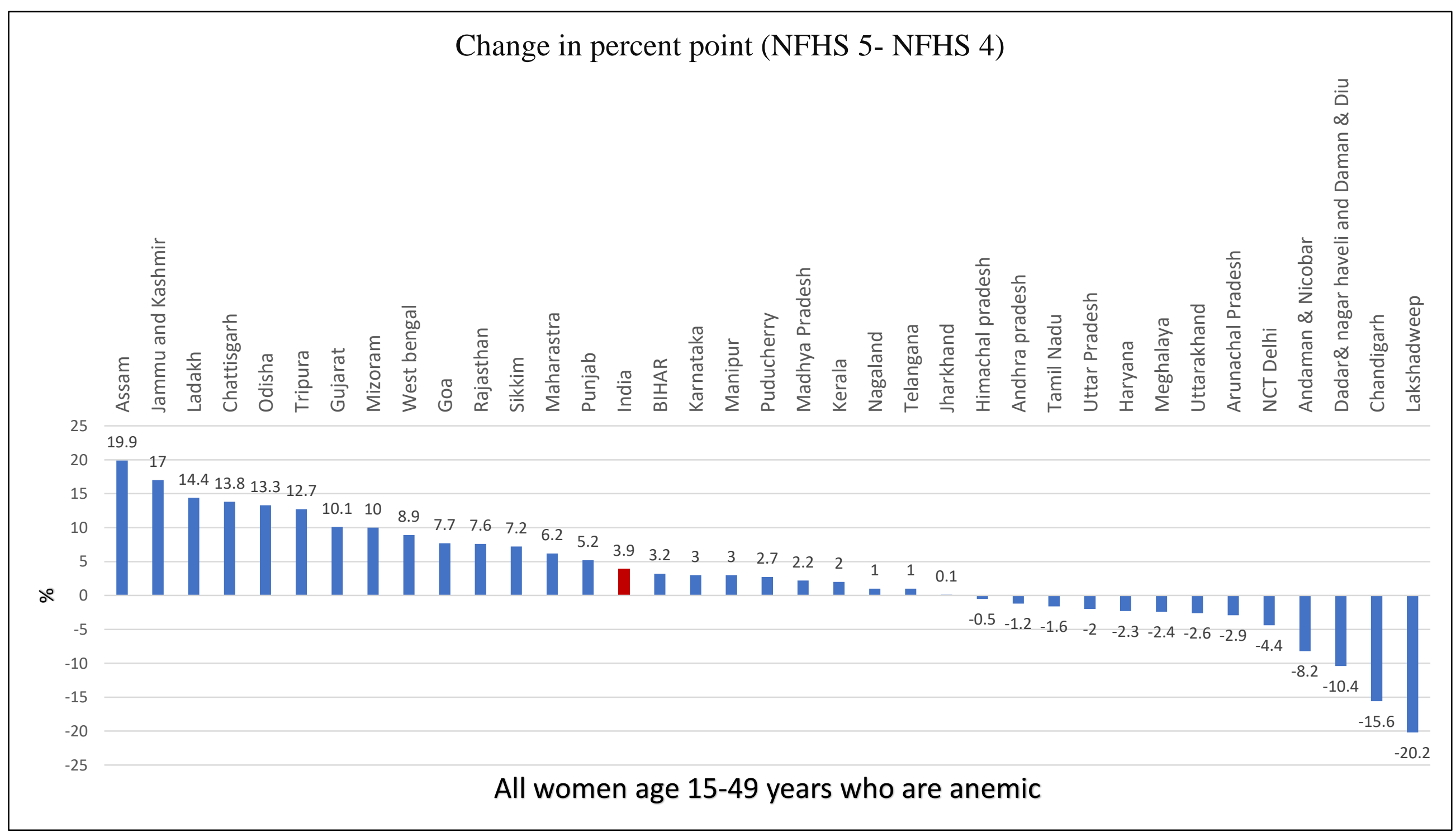


Men aged 15-49 years who are anemic

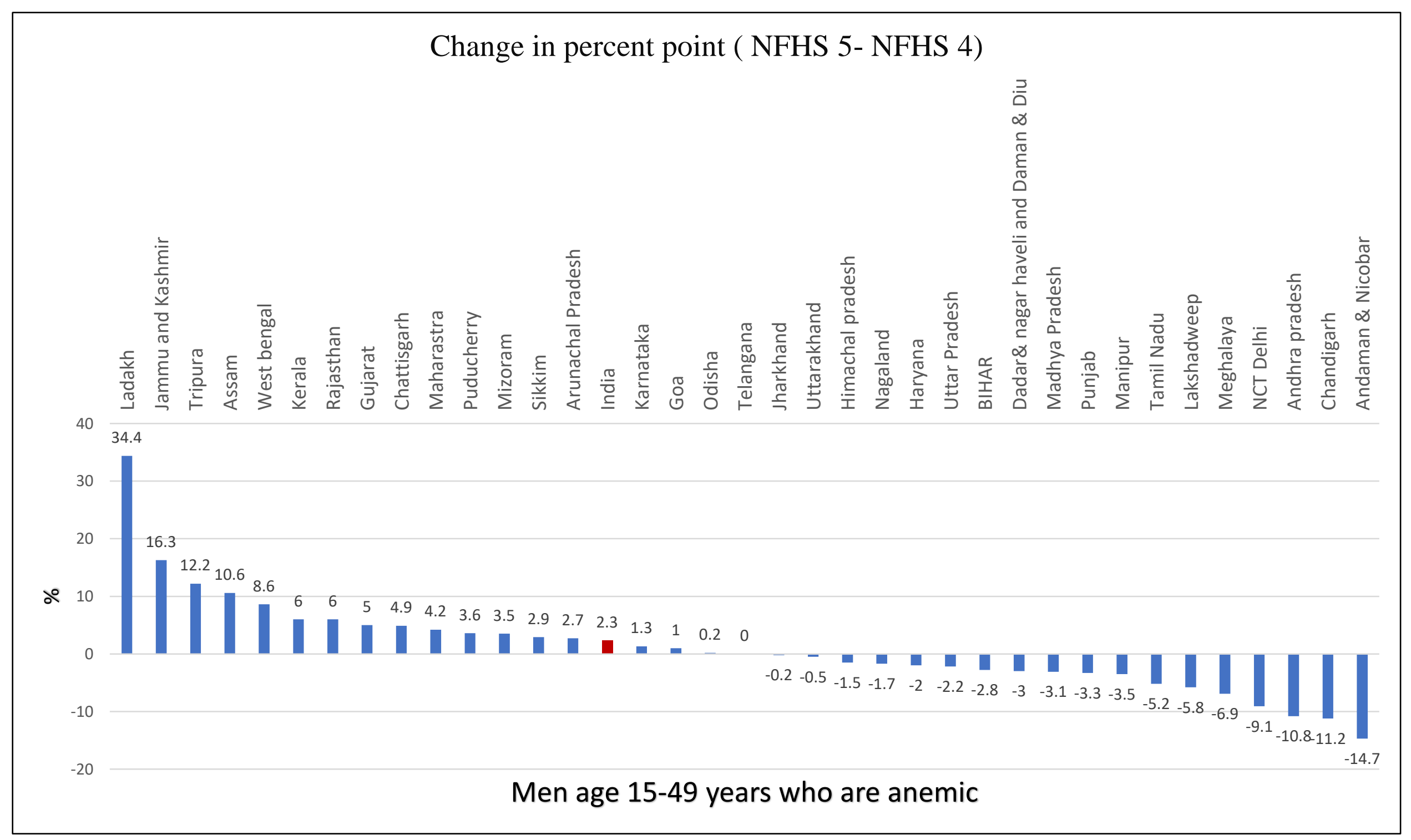


Mothers who consumed iron folic acid for 100 days or more when they were pregnant

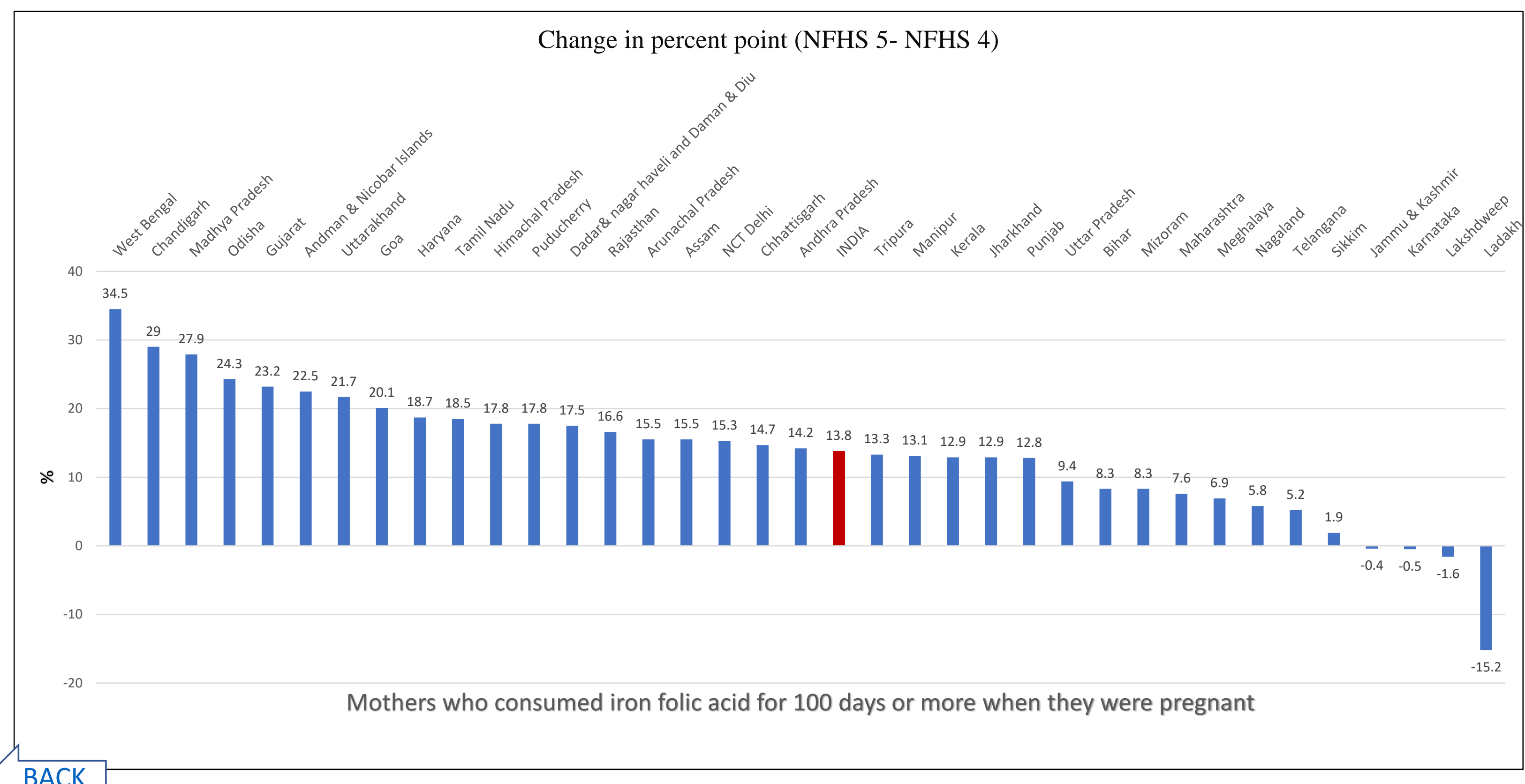

\section{BACK}


Mothers who consumed iron folic acid for 180 days or more when they were pregnant

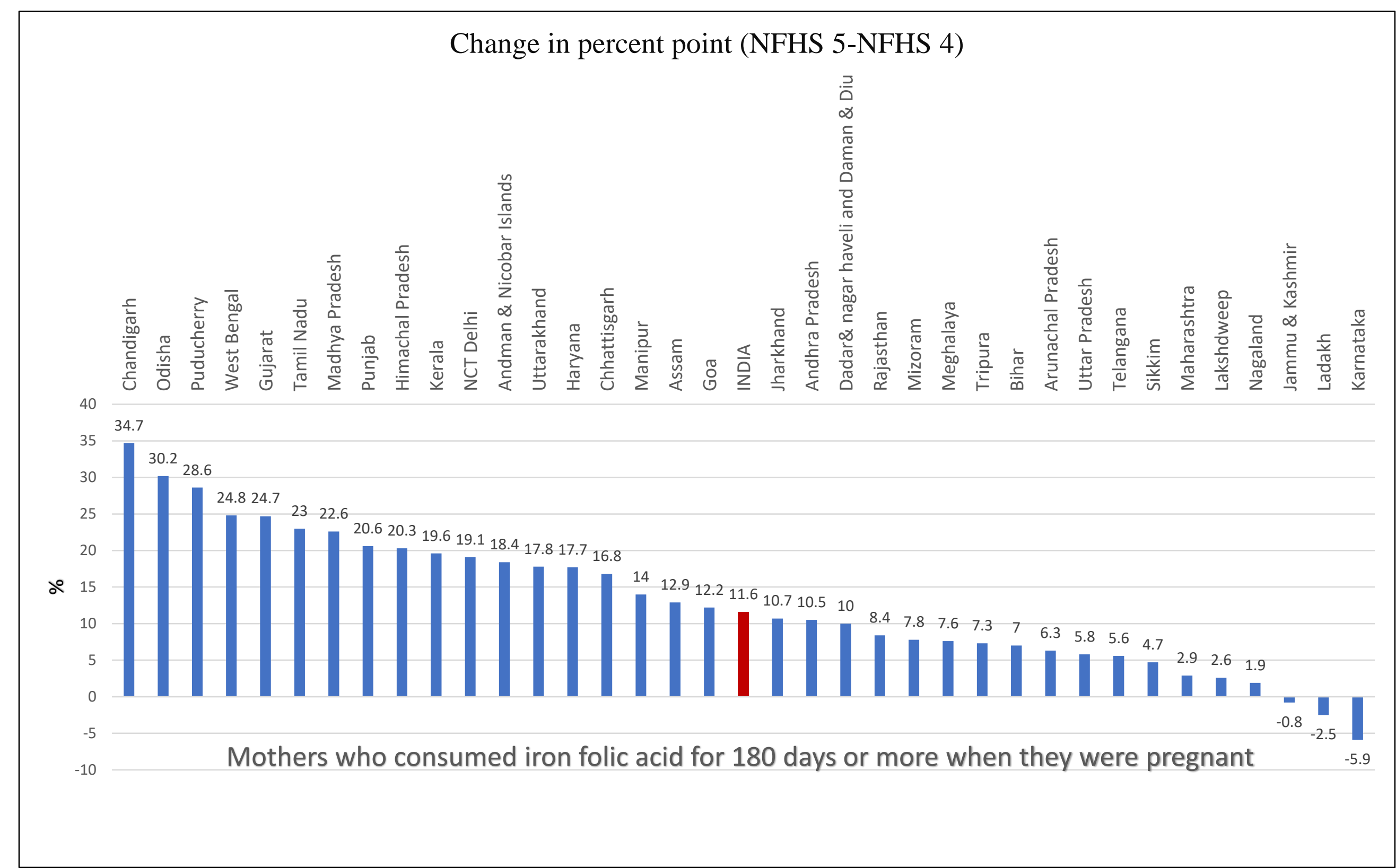


Children aged 9-35months who received a vitamin A dose in last 6 months

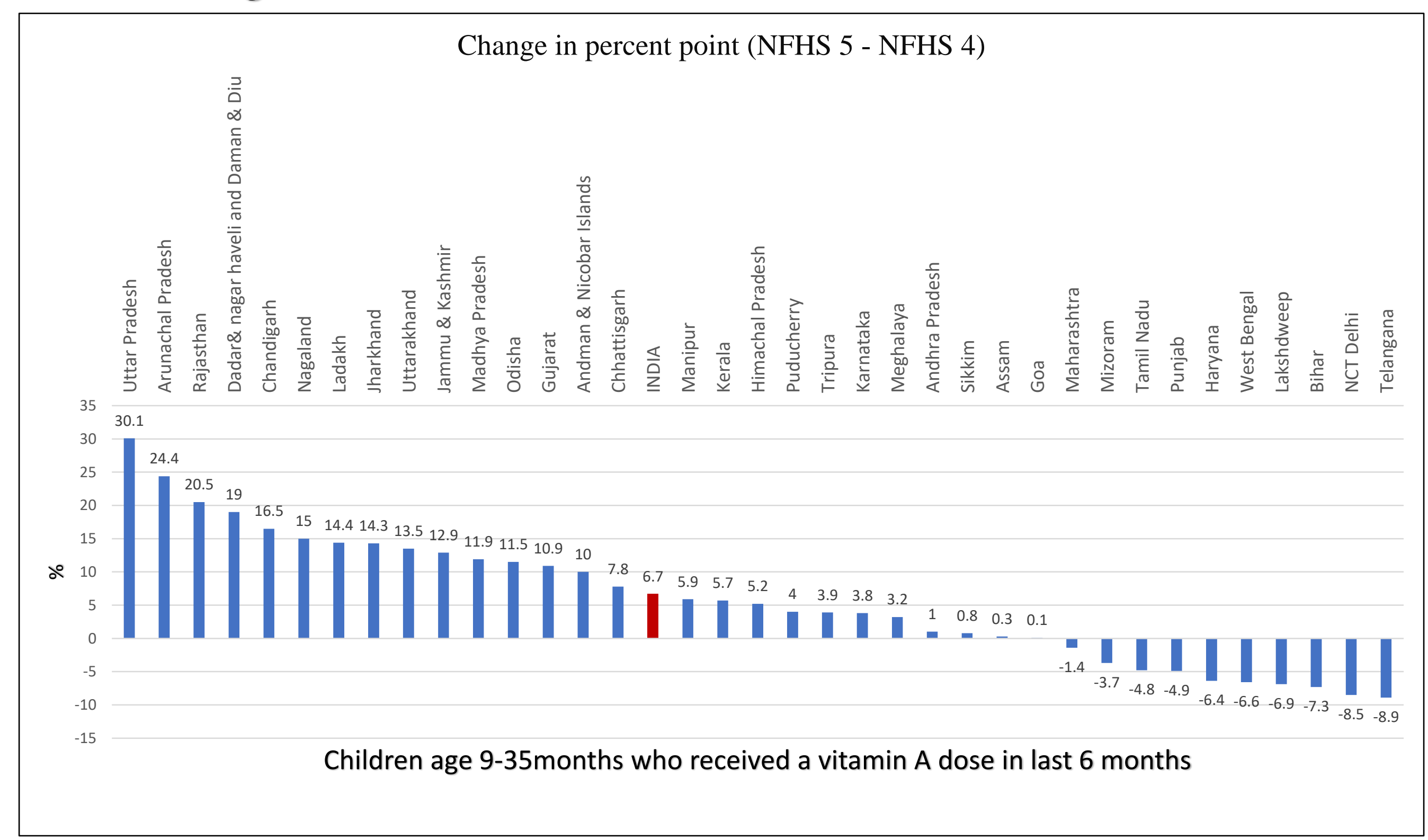




\section{Mothers who had an antenatal check-up in the first trimester}

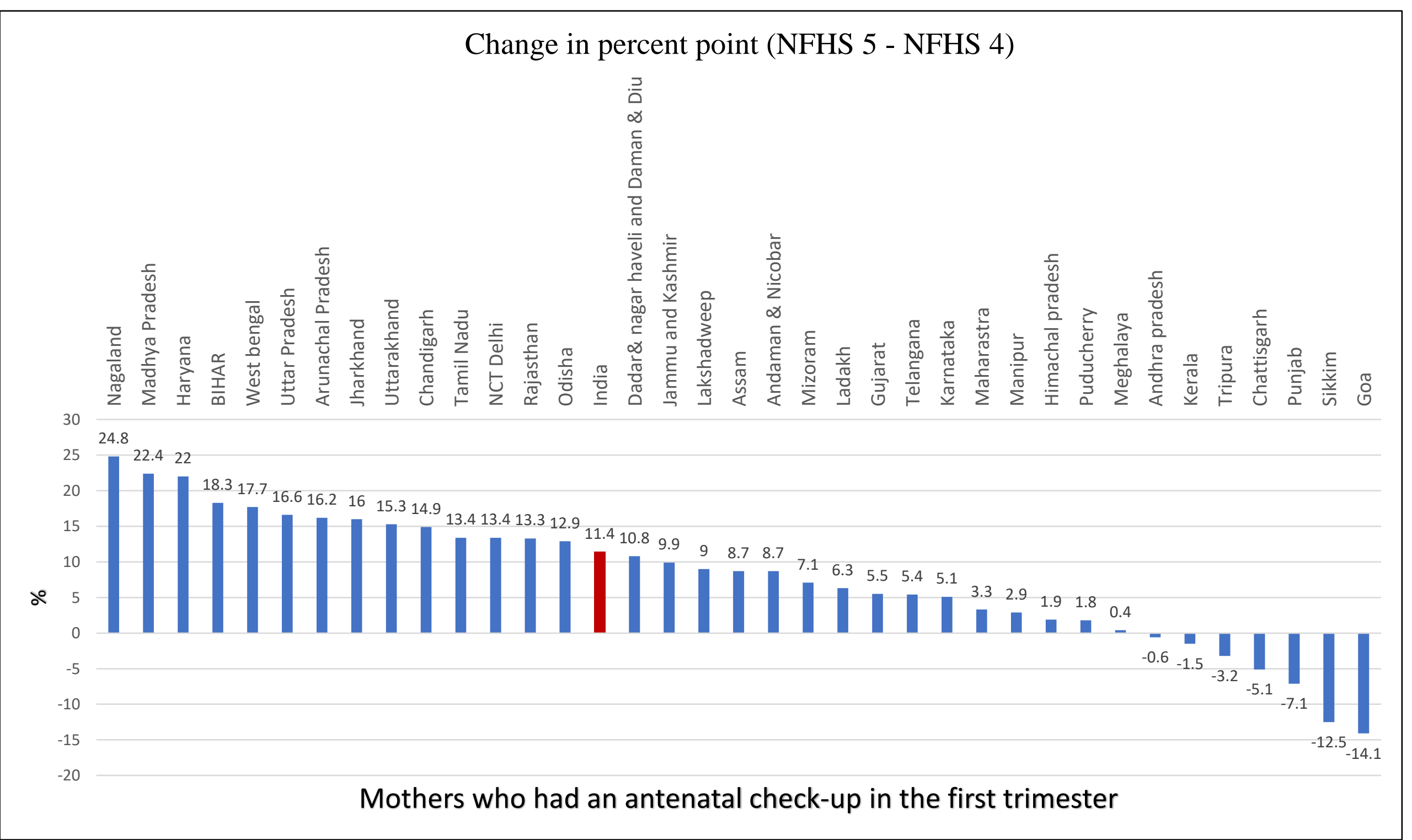


Mothers who had at least 4 antenatal check-up in the first trimester

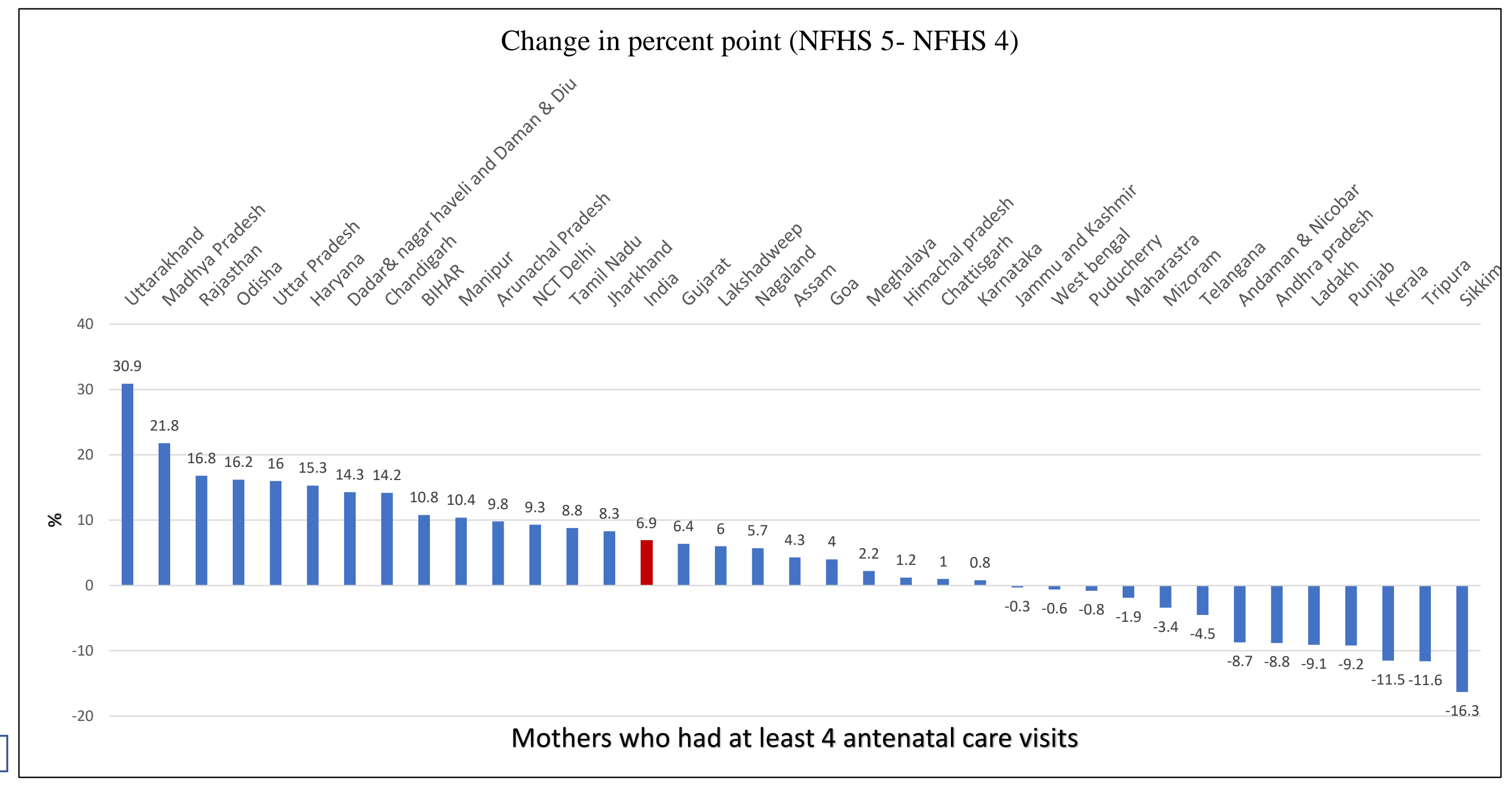




\section{Mothers who received postnatal care}

Change in percent point (NFHS 5- NFHS 4)
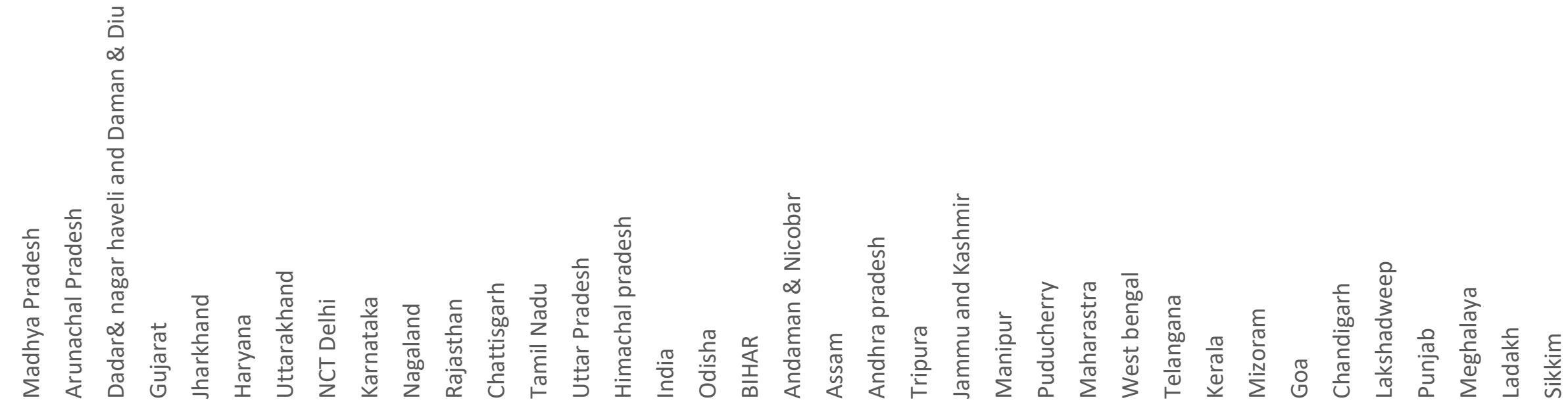

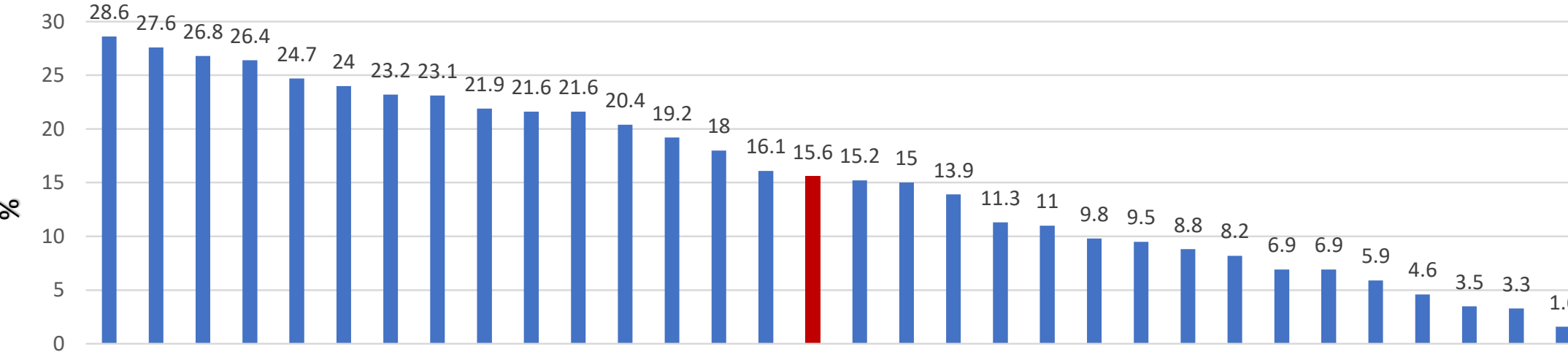

Mothers who recieved post natal care within 2 days of delivery 


\section{Institutional births}

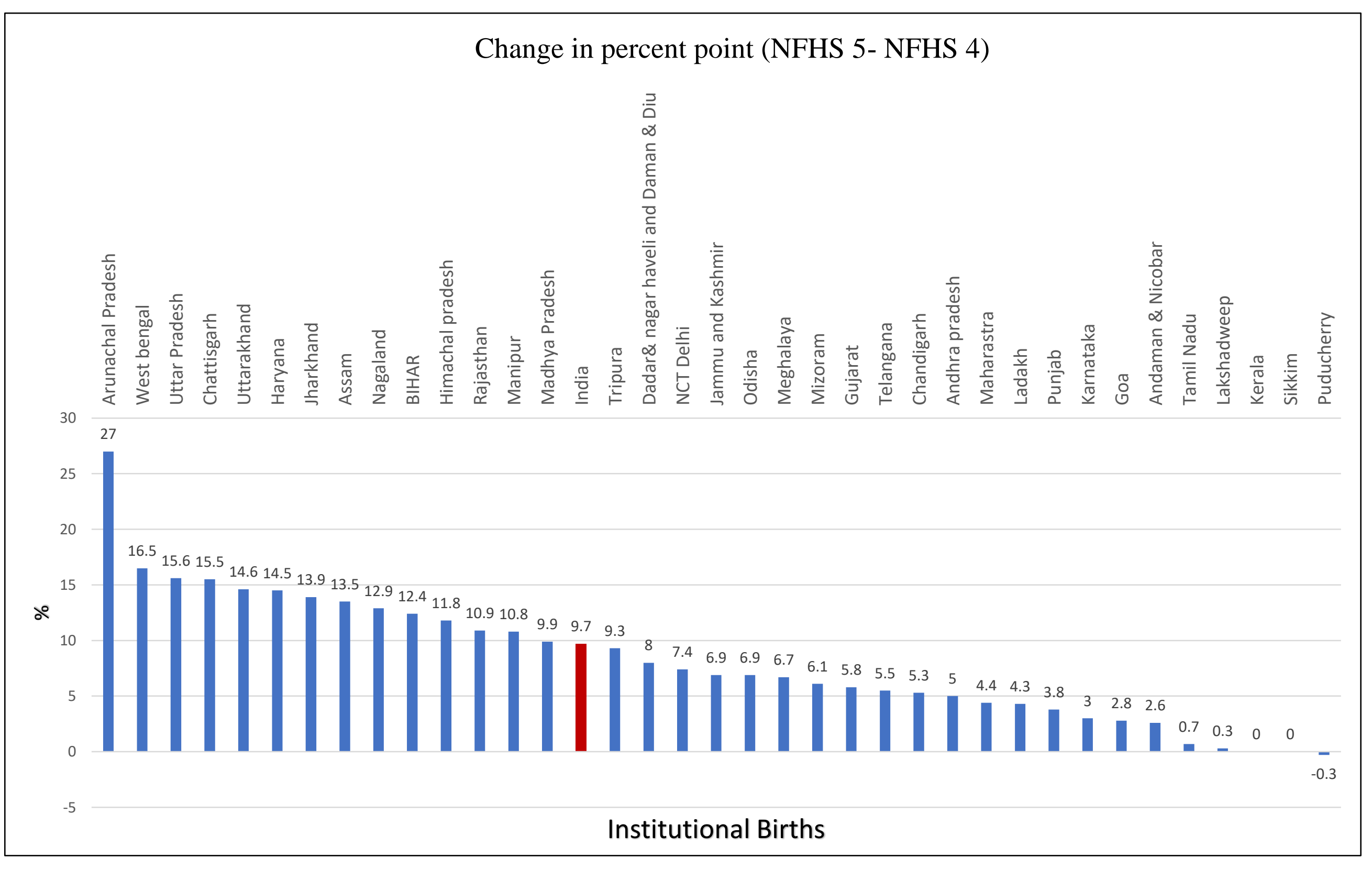


Births delivered by caesarean section

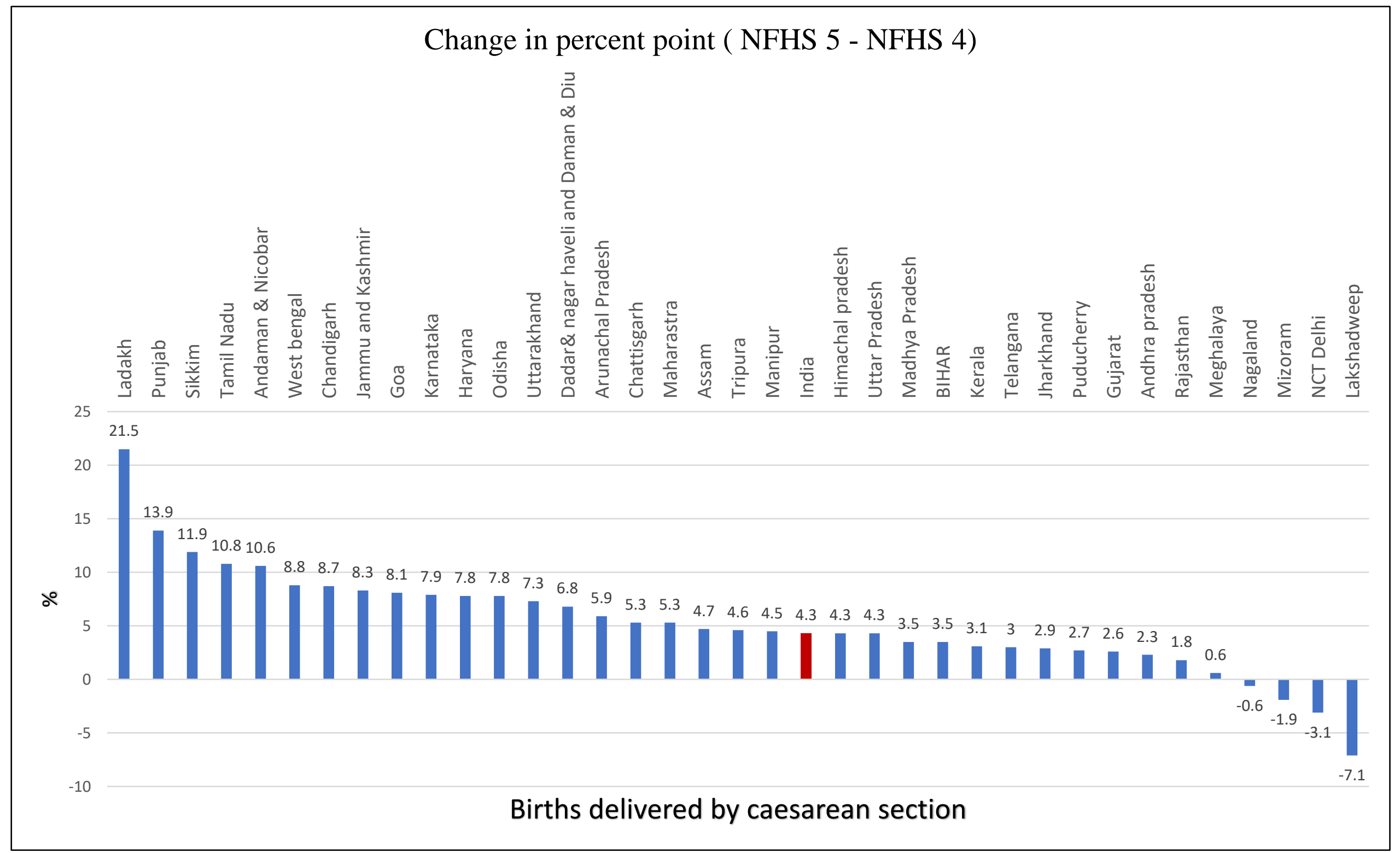


Prevalence of diarrhea in the 2 weeks preceding the survey

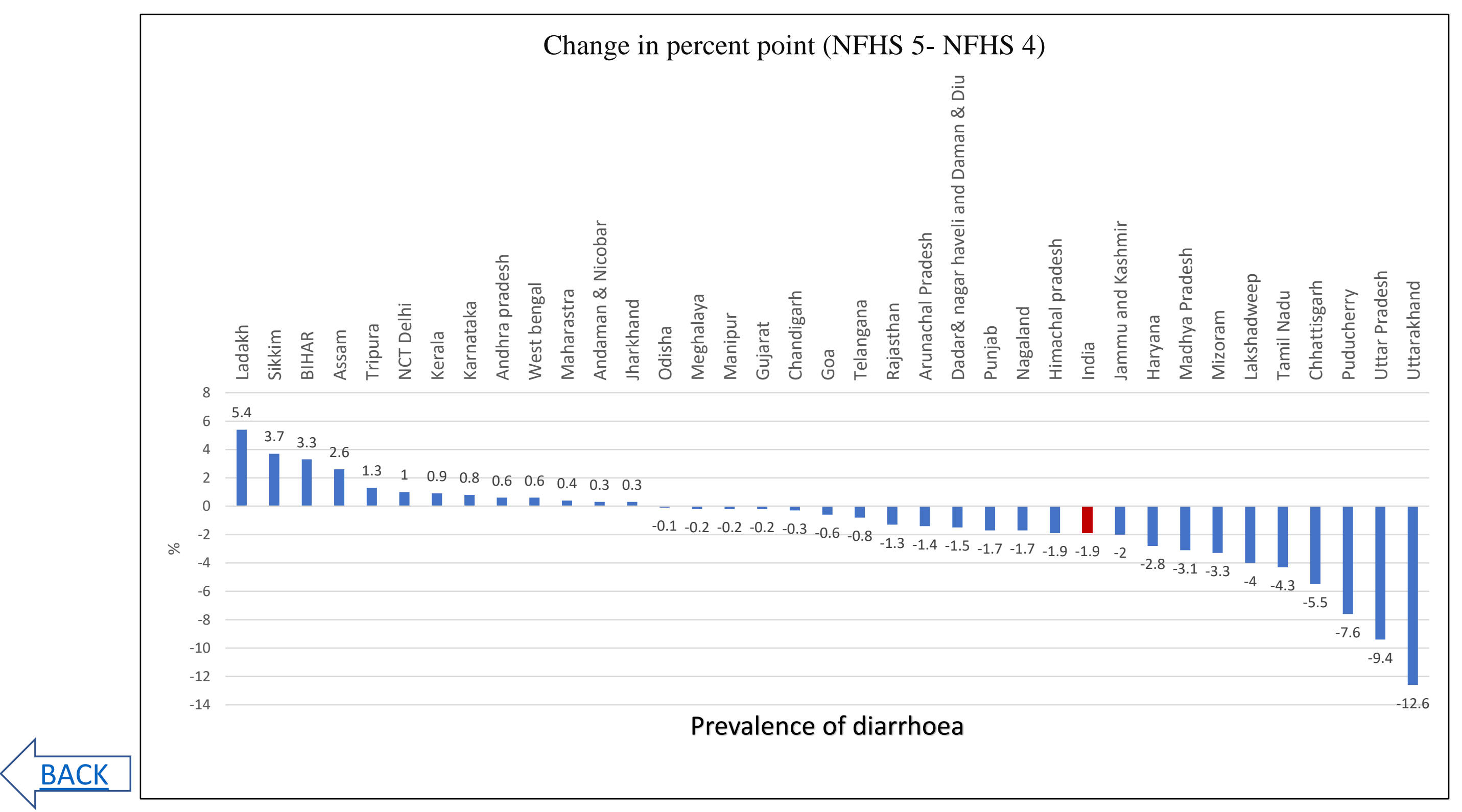




\section{Children with diarrhoea who received ORS}

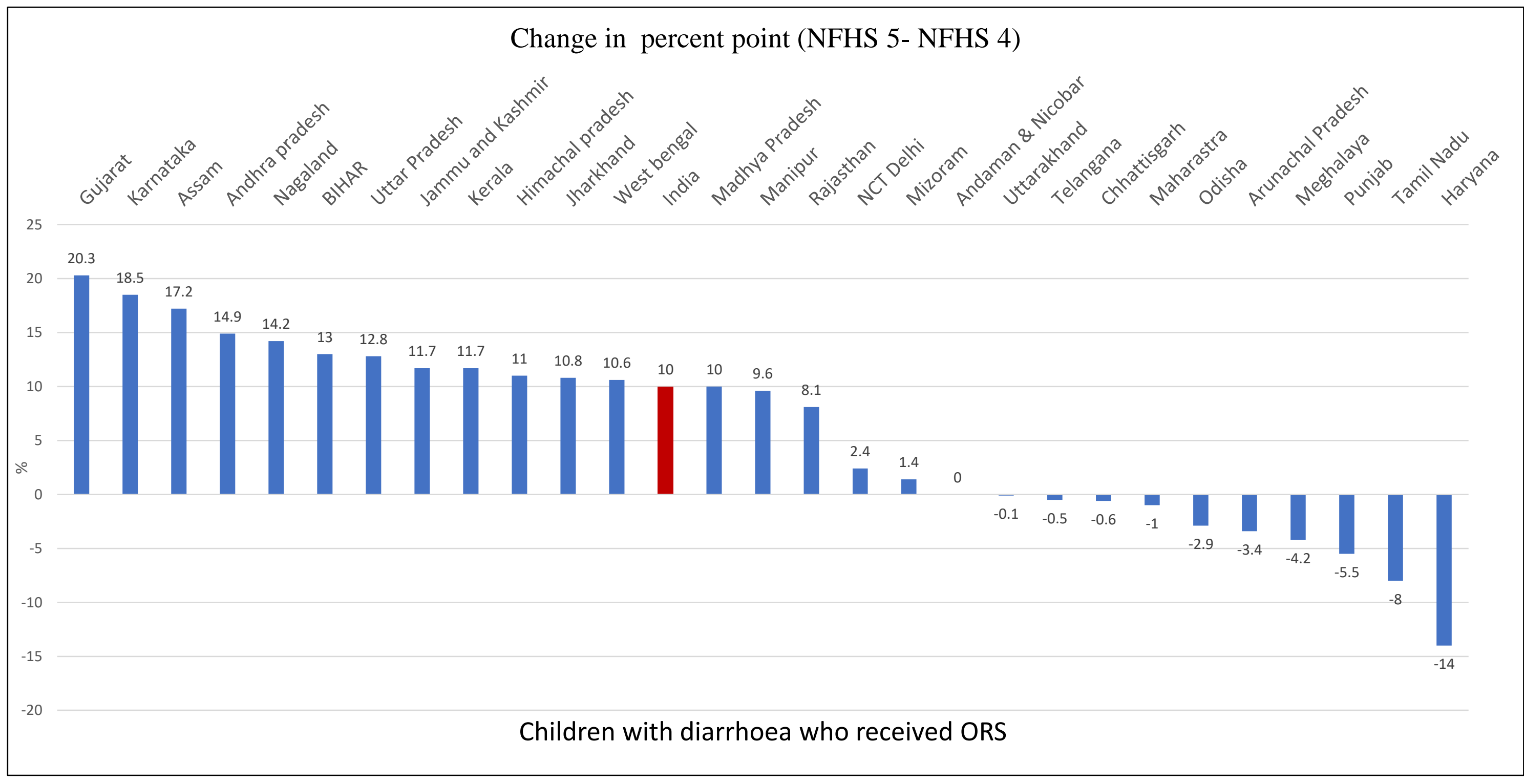




\section{Children with Diarrhoea who received Zinc}

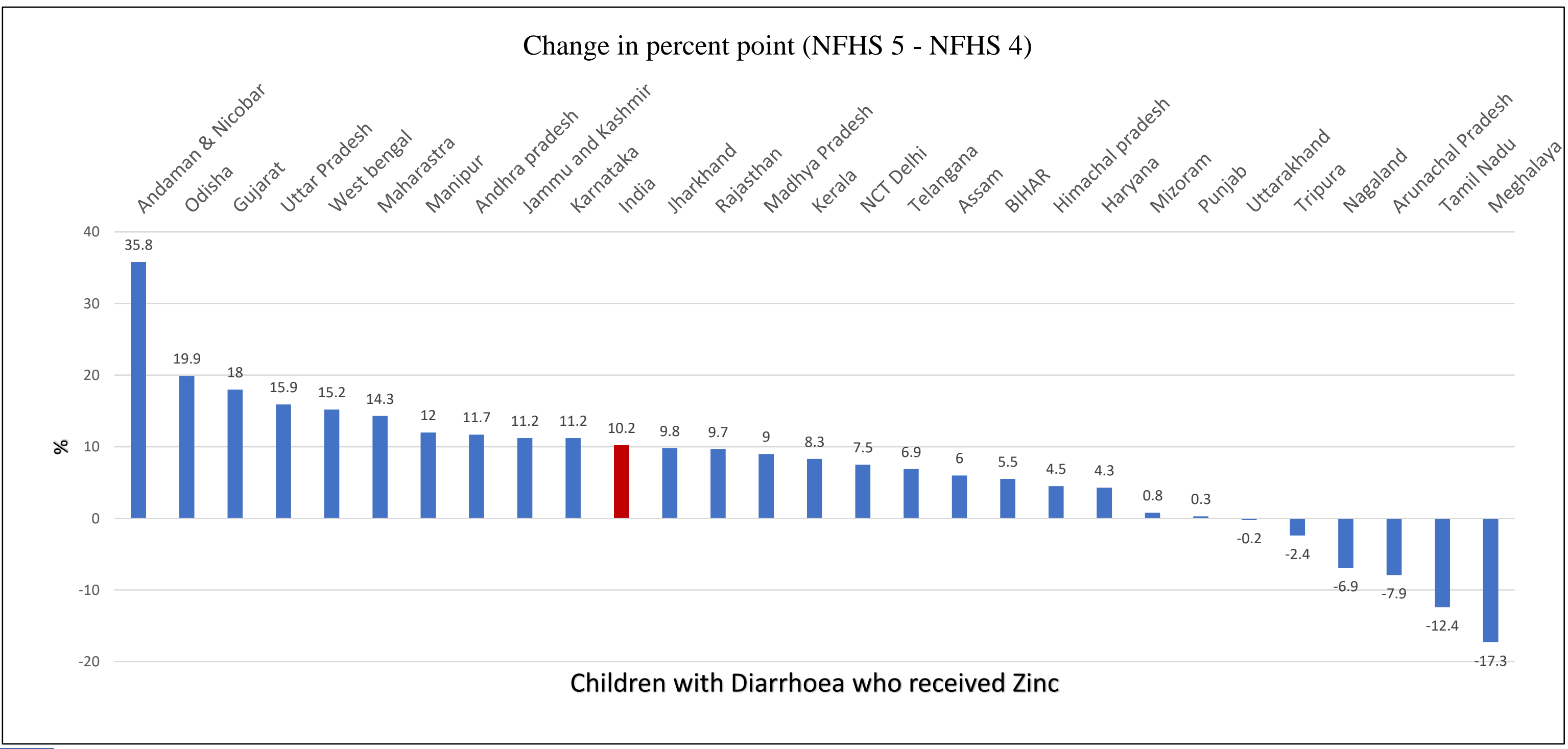




\section{Prevalence of symptoms of ARI}

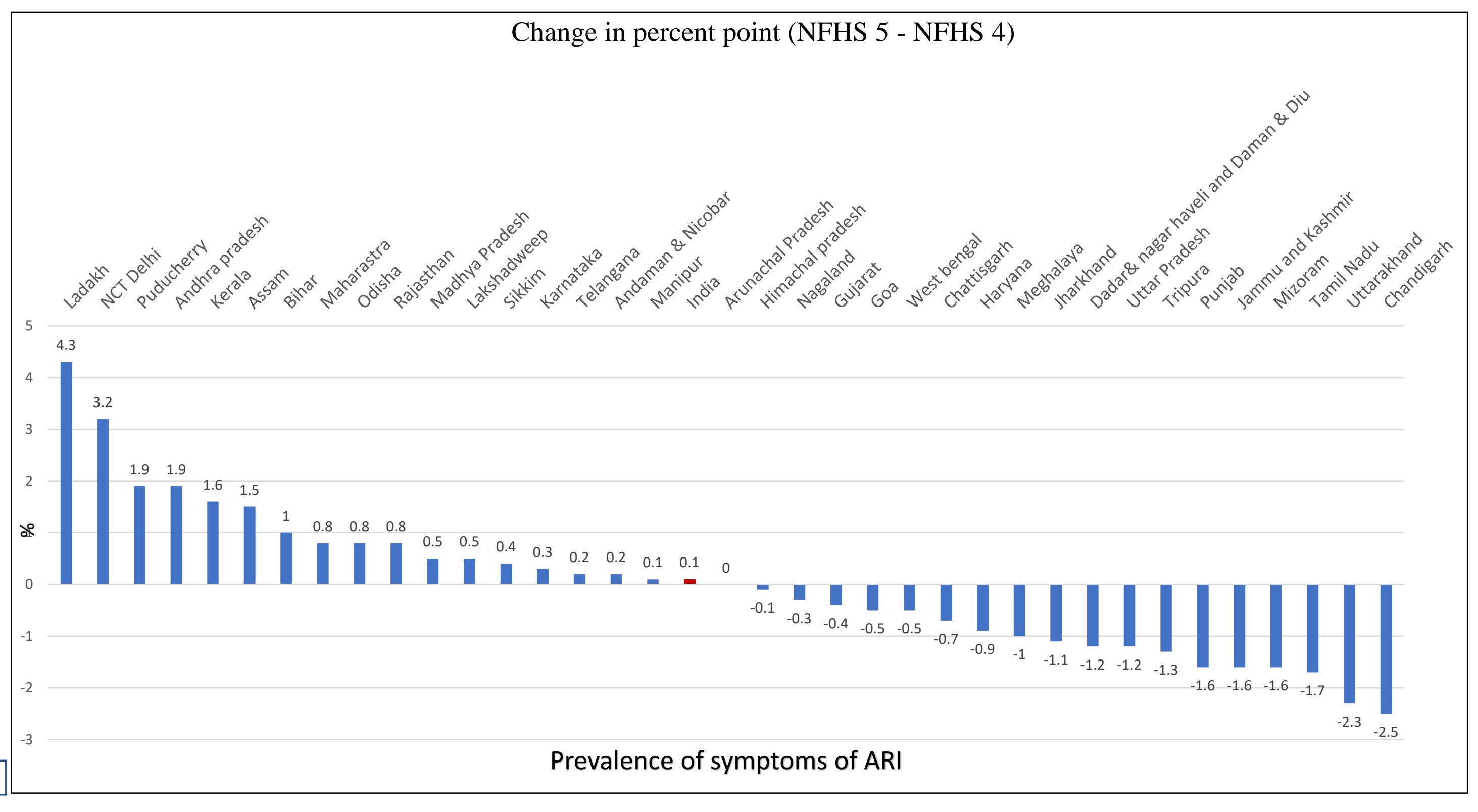


Children with symptoms of ARI taken to health facility

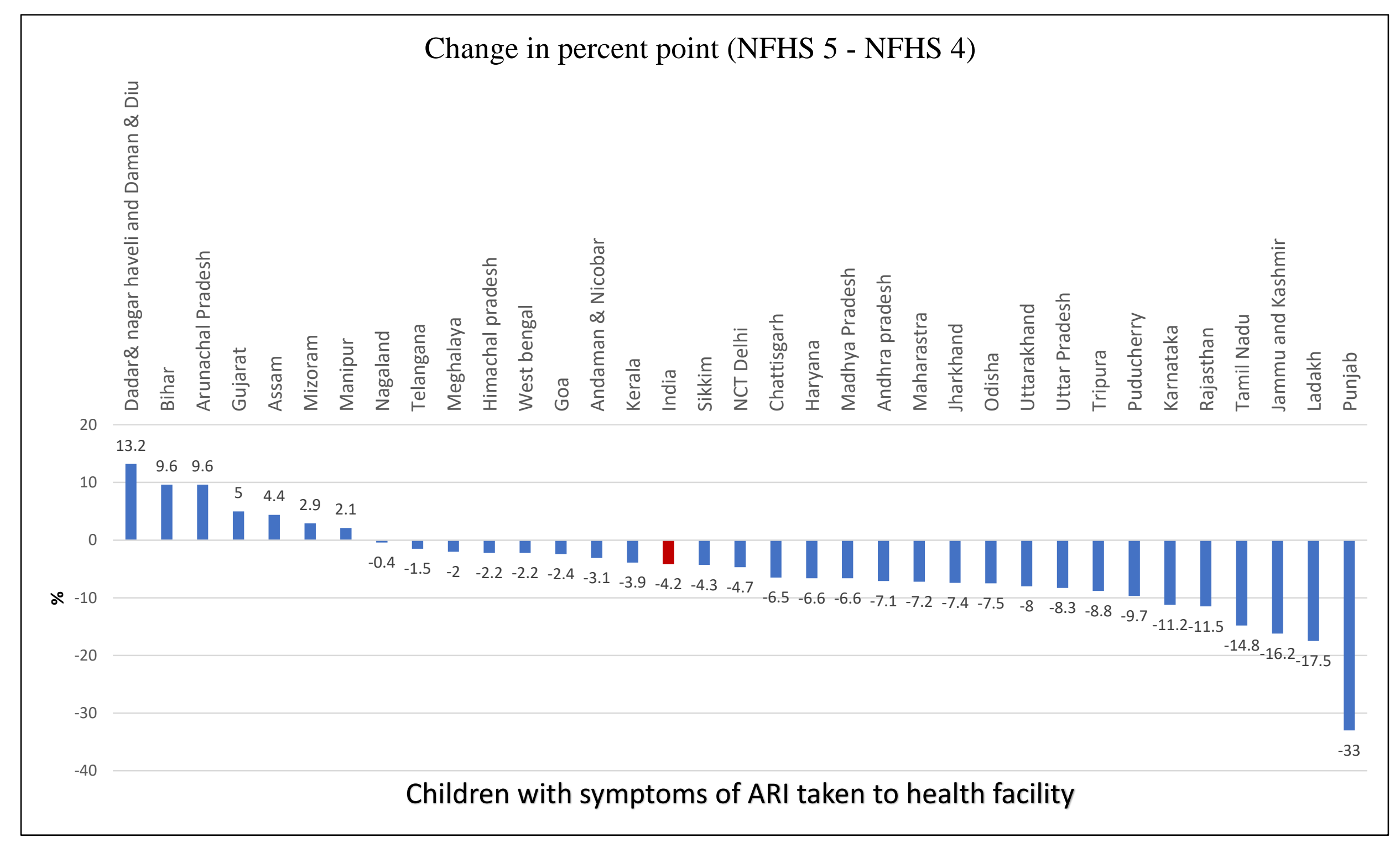




\section{Children age 12-23 months fully vaccinated}

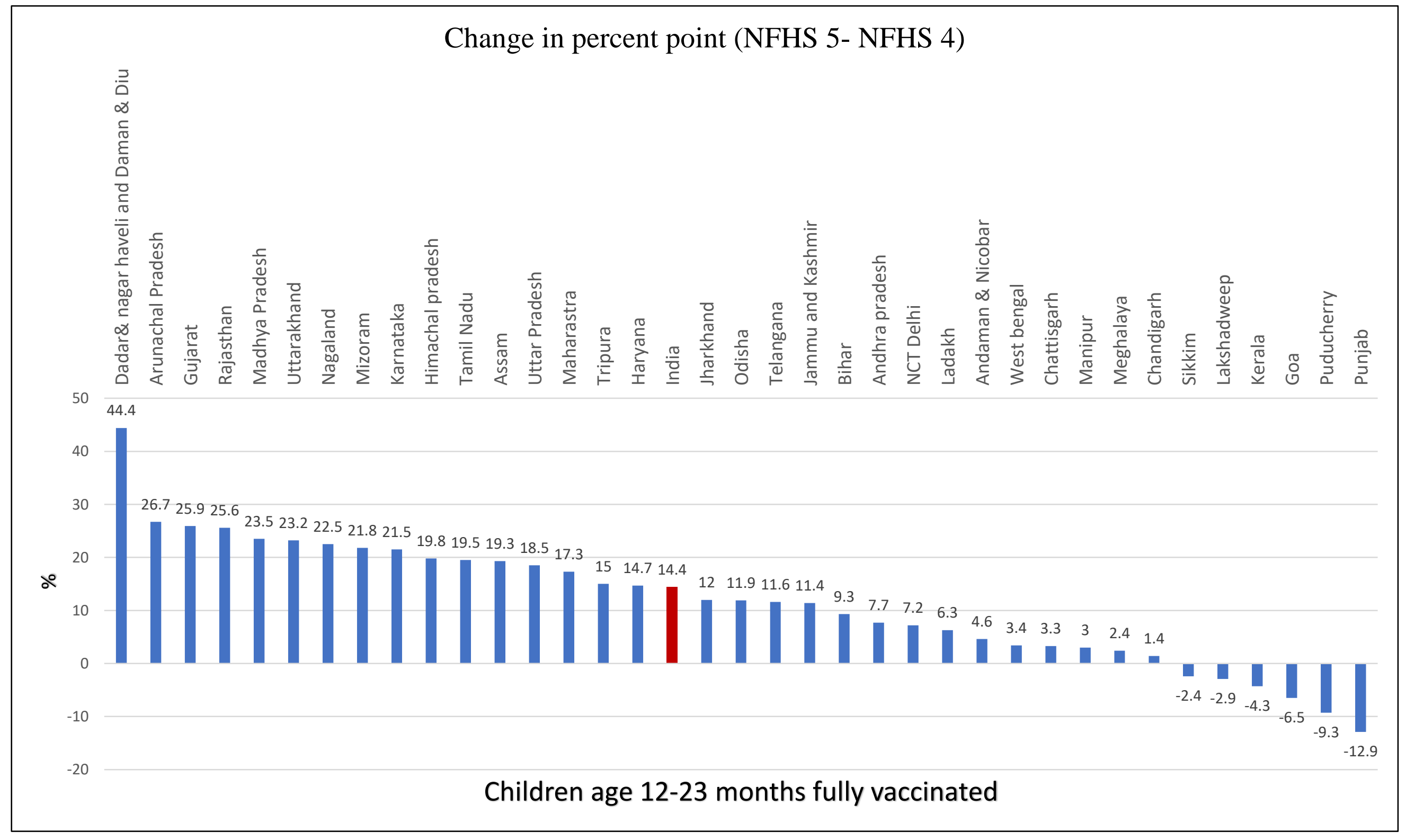


Children under age 3 years breastfed within one hour of birth

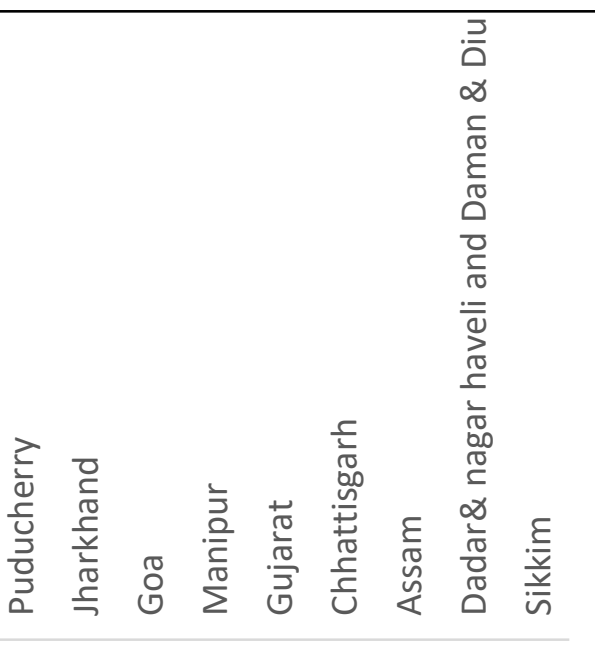

40
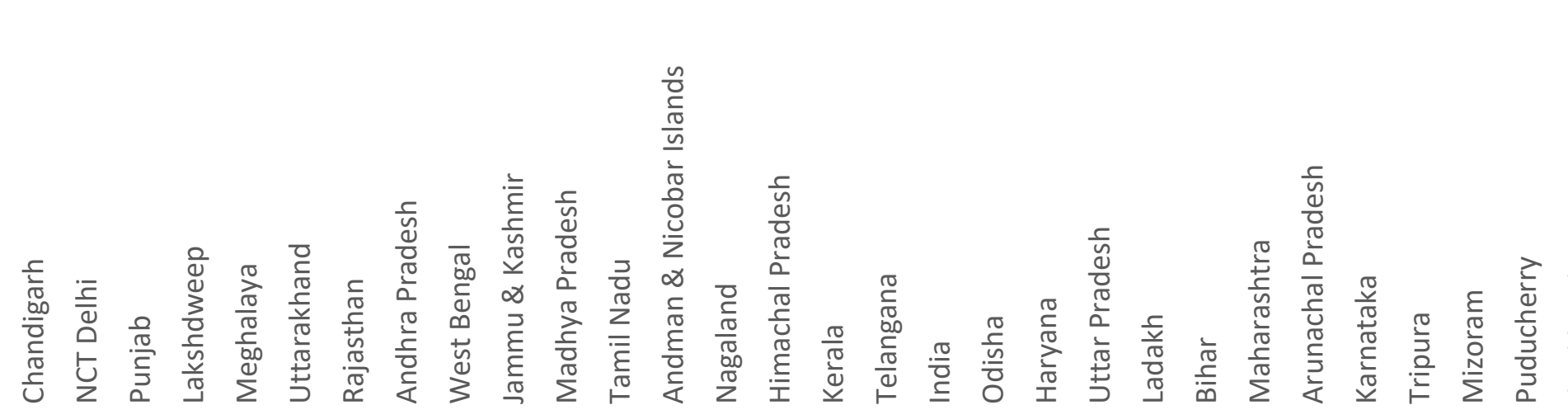

(23.2 22.4

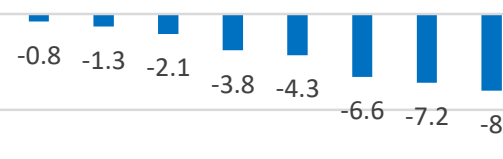




\section{Children under age 6 months exclusively breastfed}

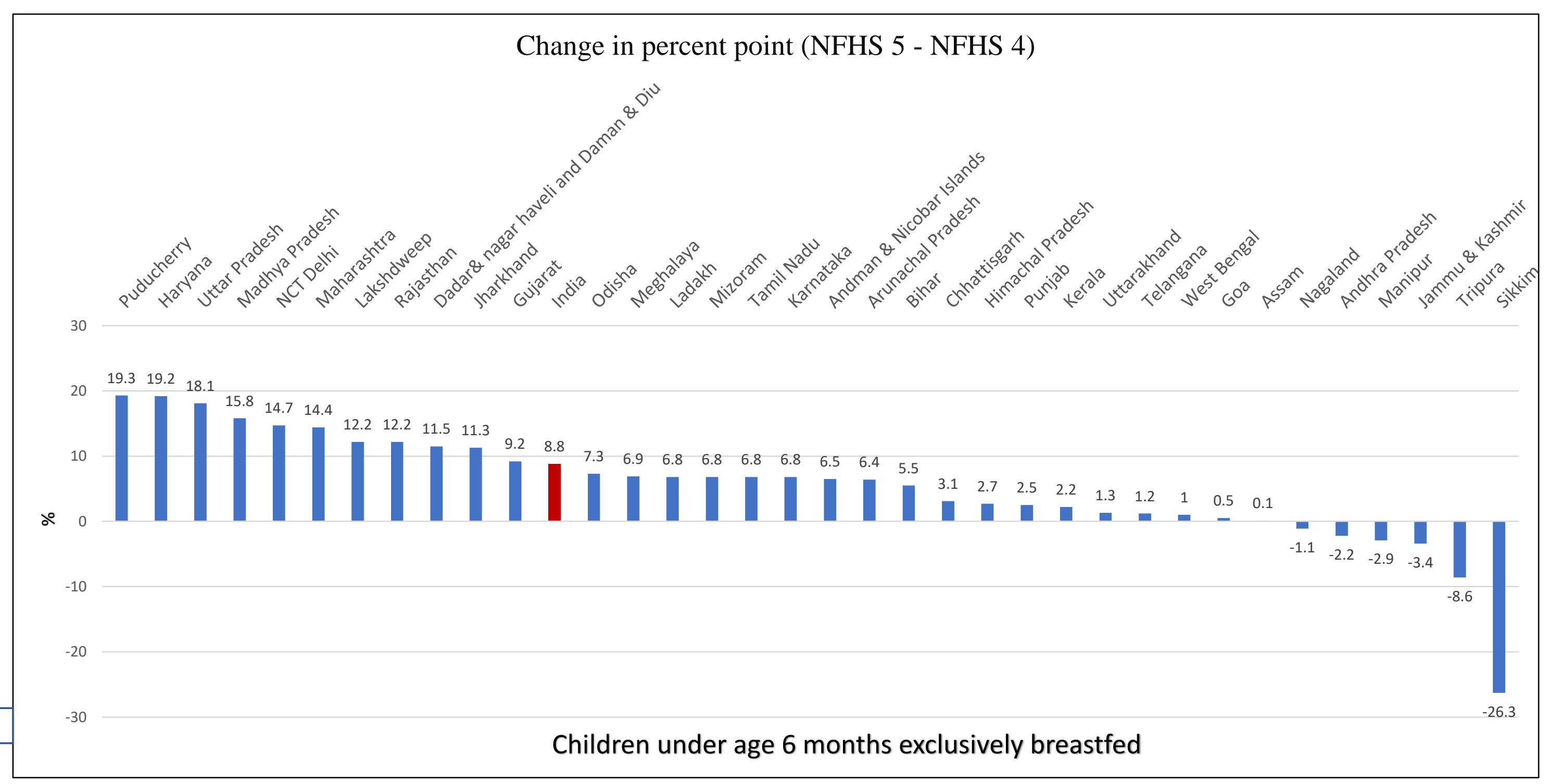


Children age 6-8 months receiving solid or semi-solid food and breastmilk

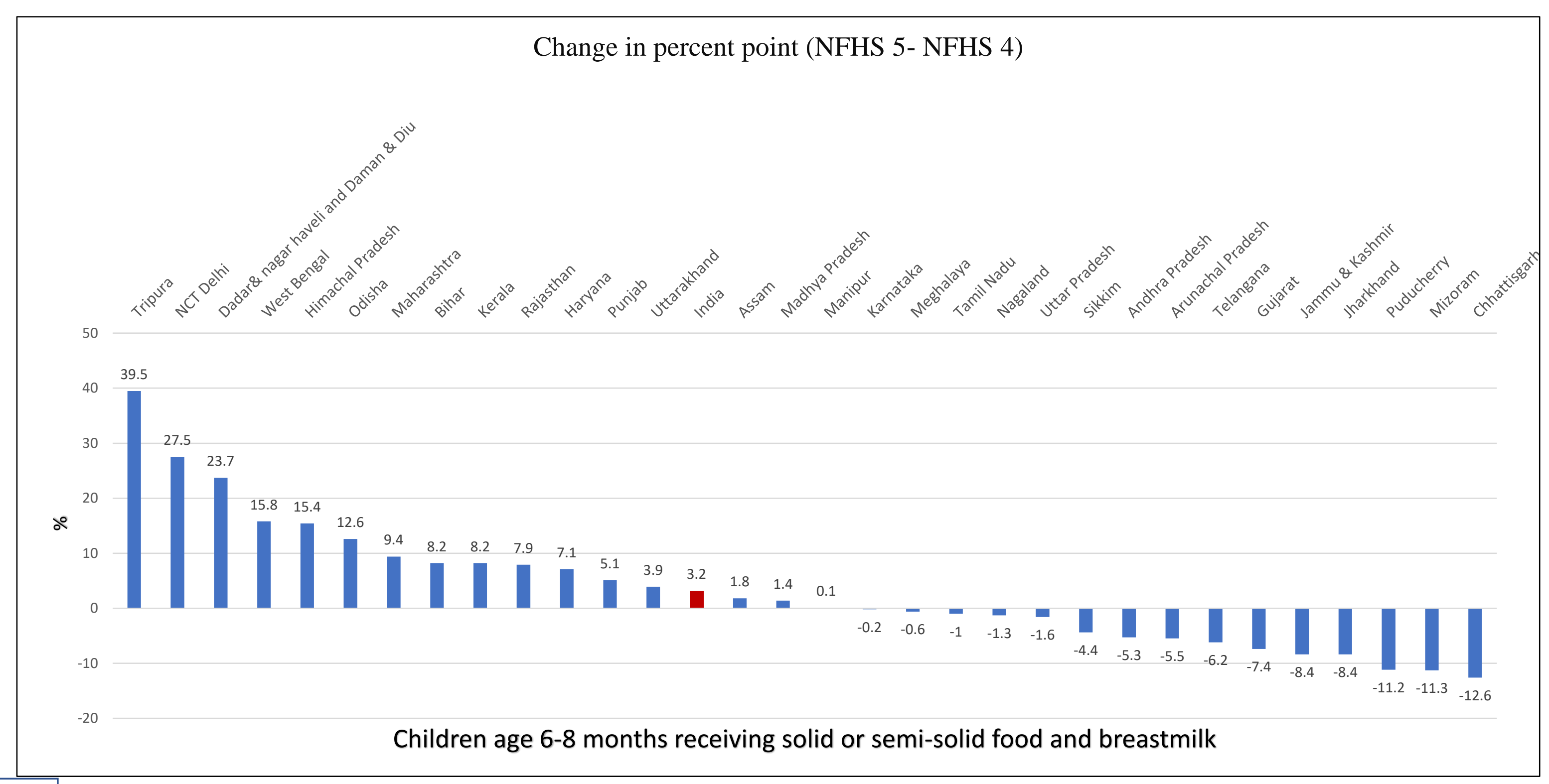


Total children age 6-23 months receiving an adequate diet

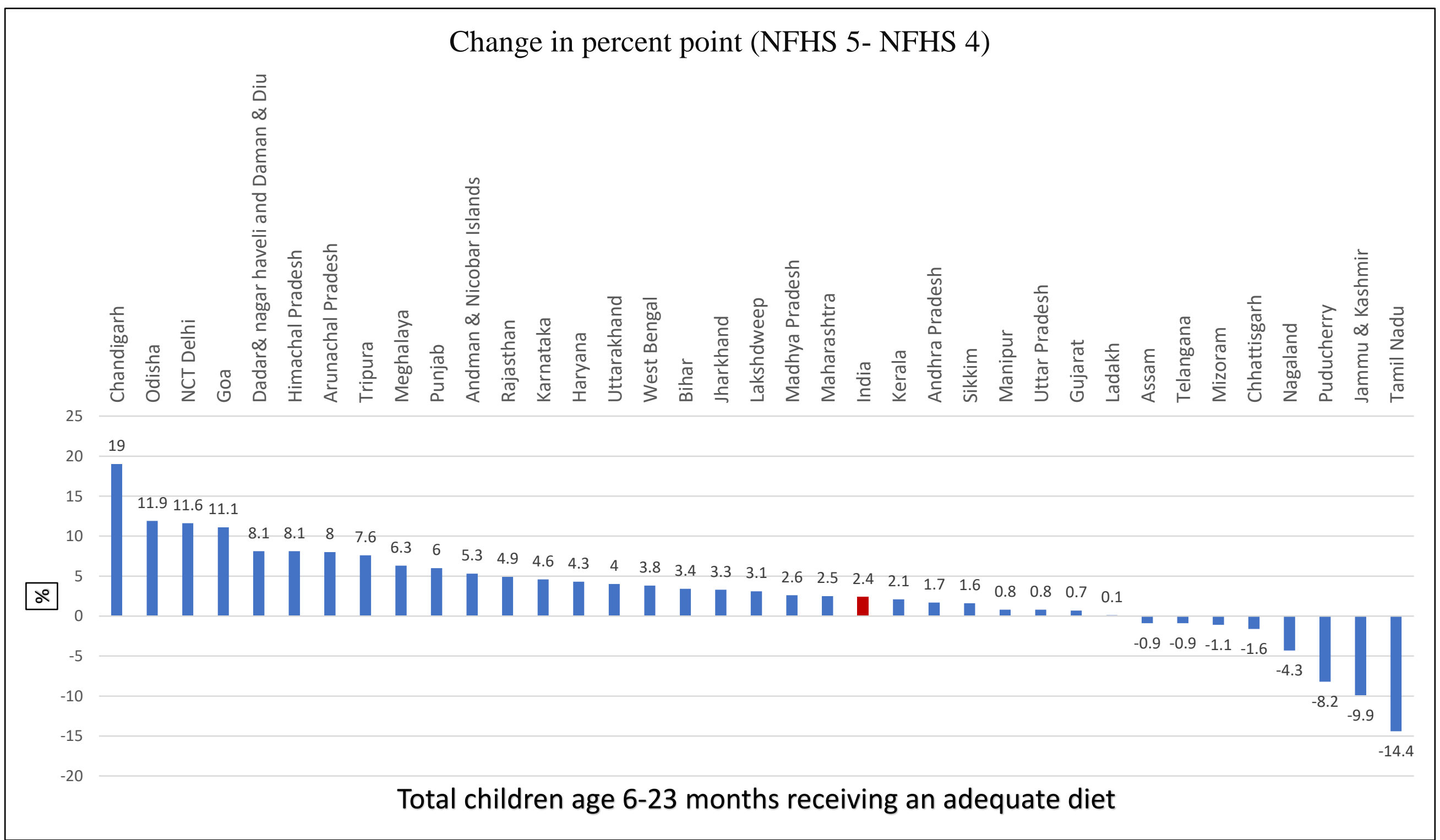




\section{Population living in households with electricity}

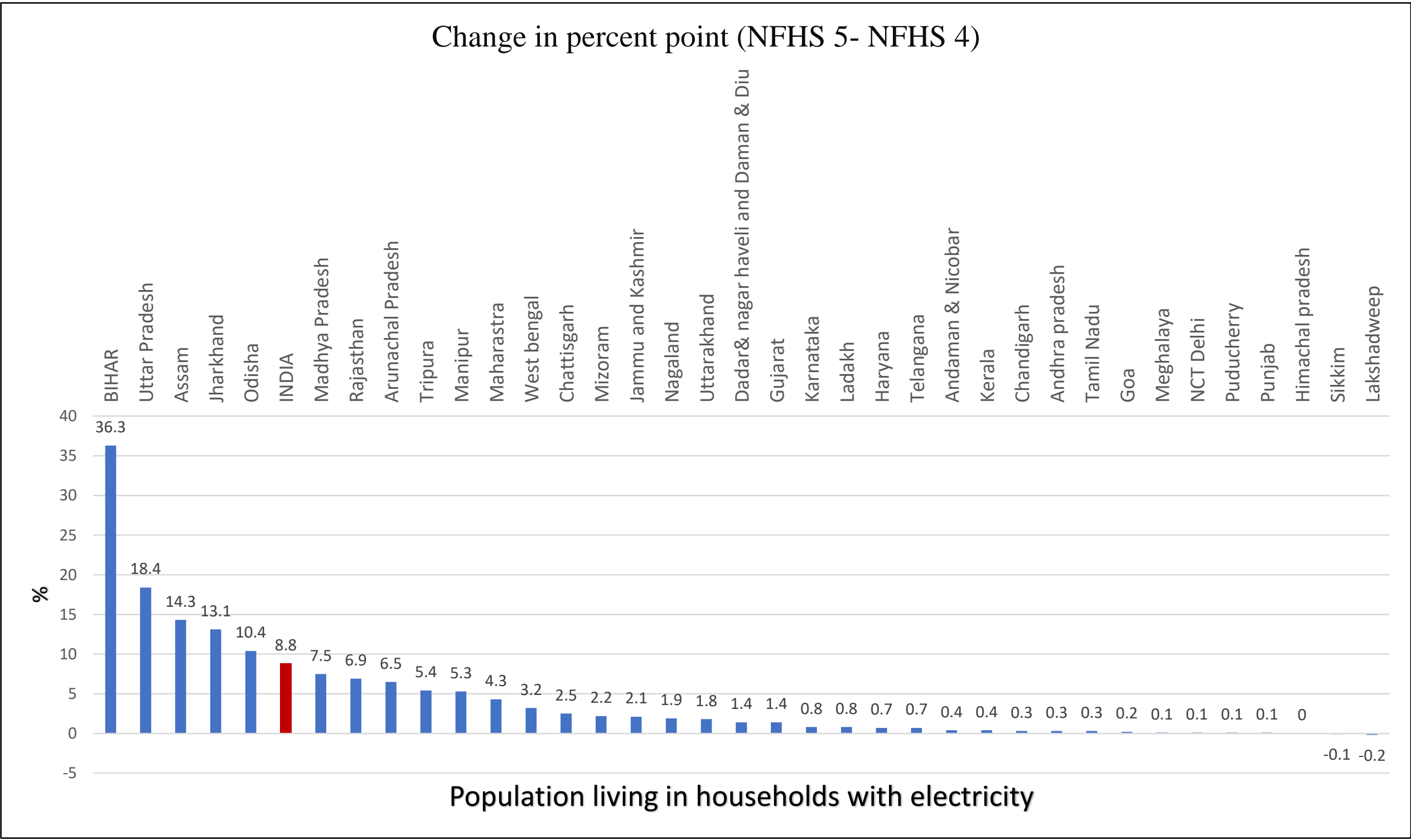


Population living in households with an improved drinking-water source

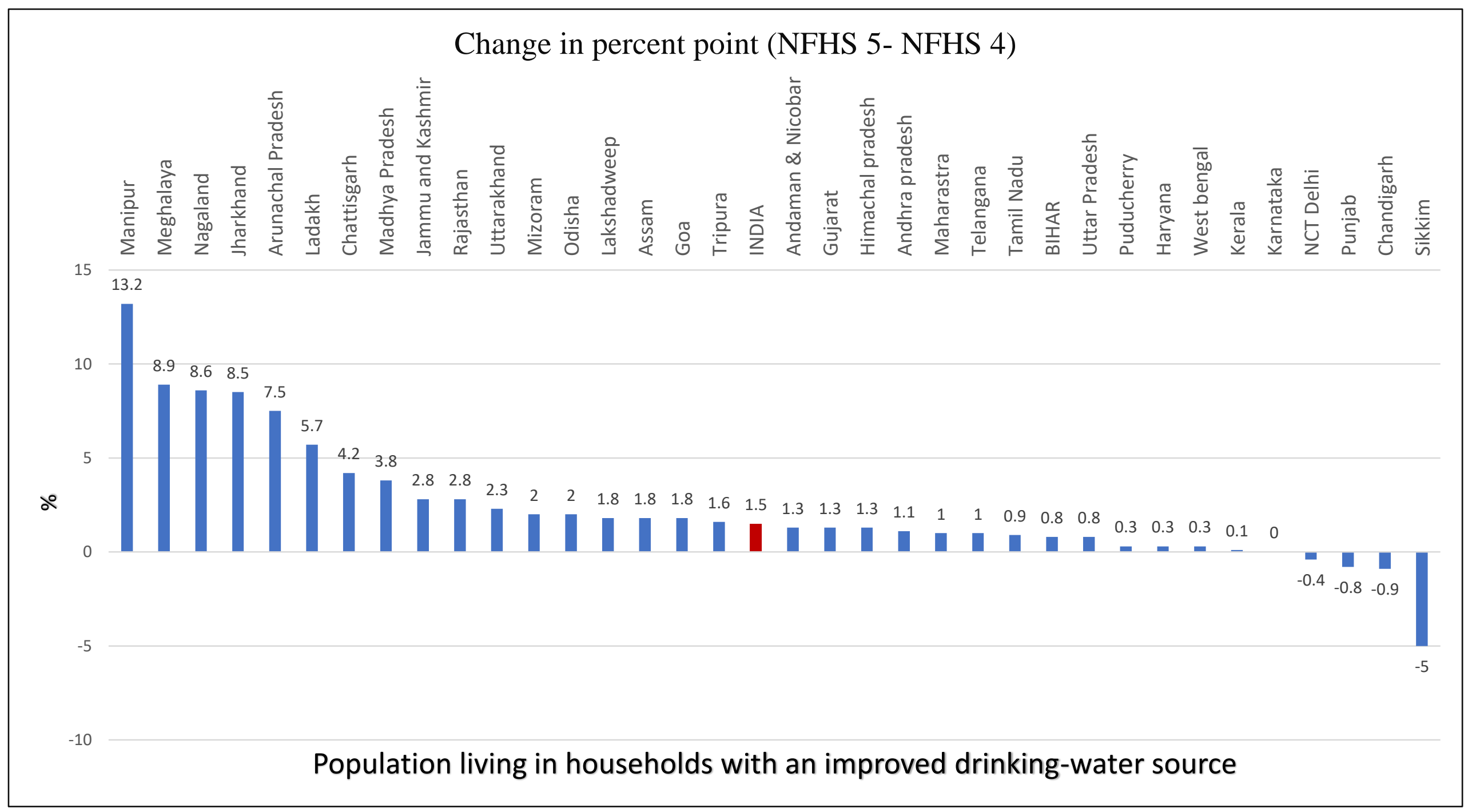


Population living in households that use an improved sanitation facility

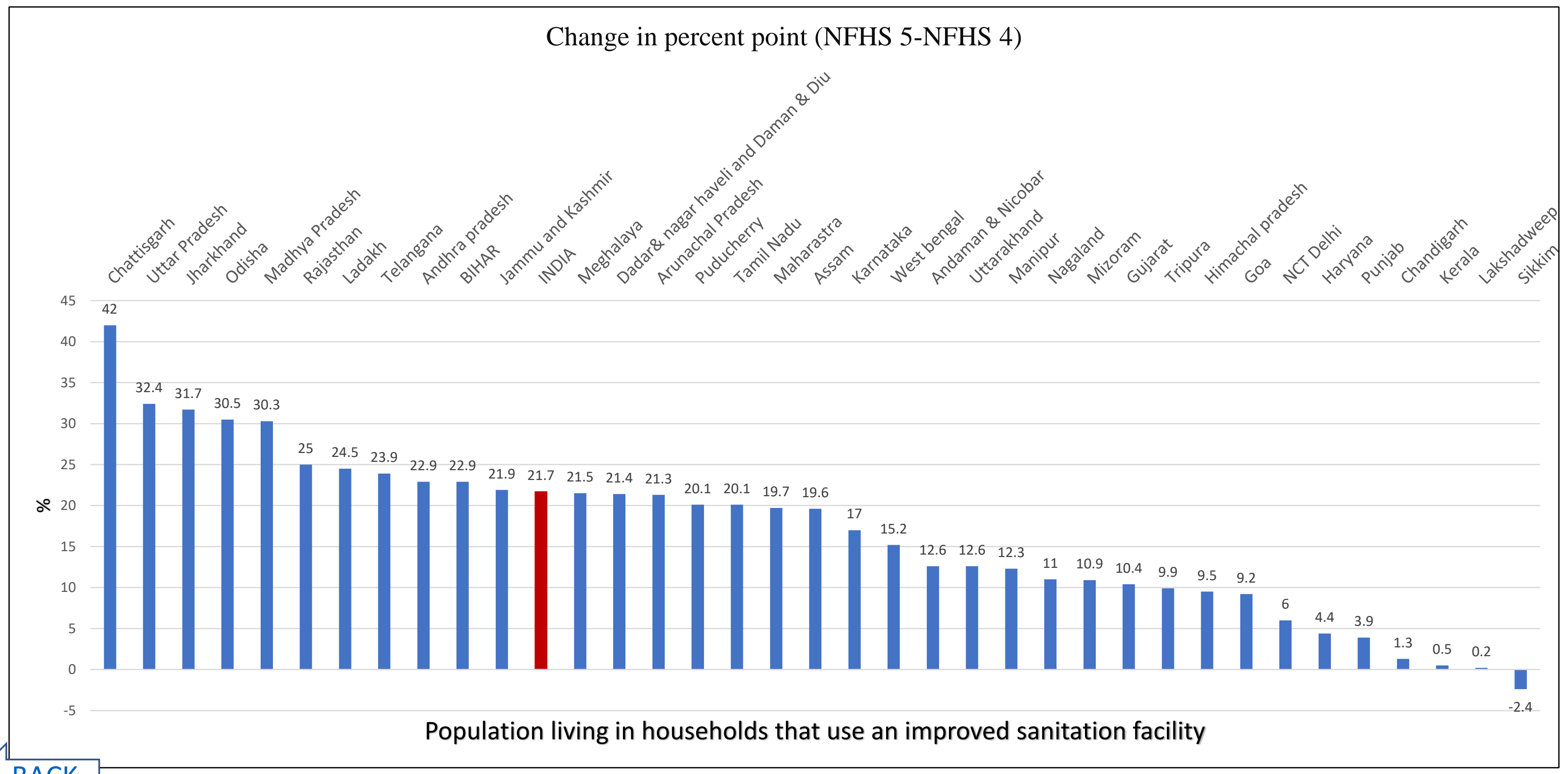


Sex ratio of the total population (females per 1000 males)

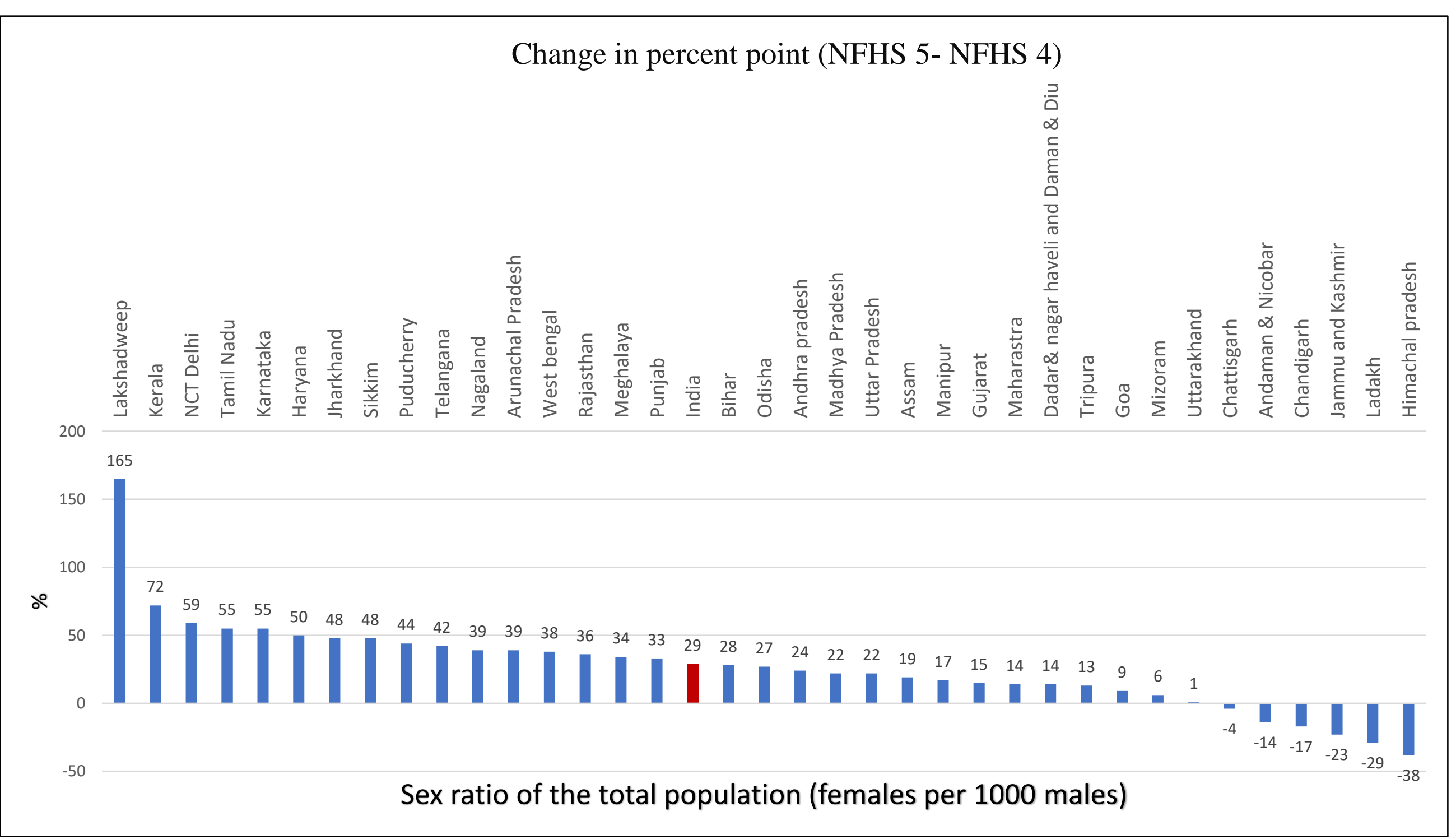




\section{Change in percent point (NFHS 5- NFHS 4)}

20

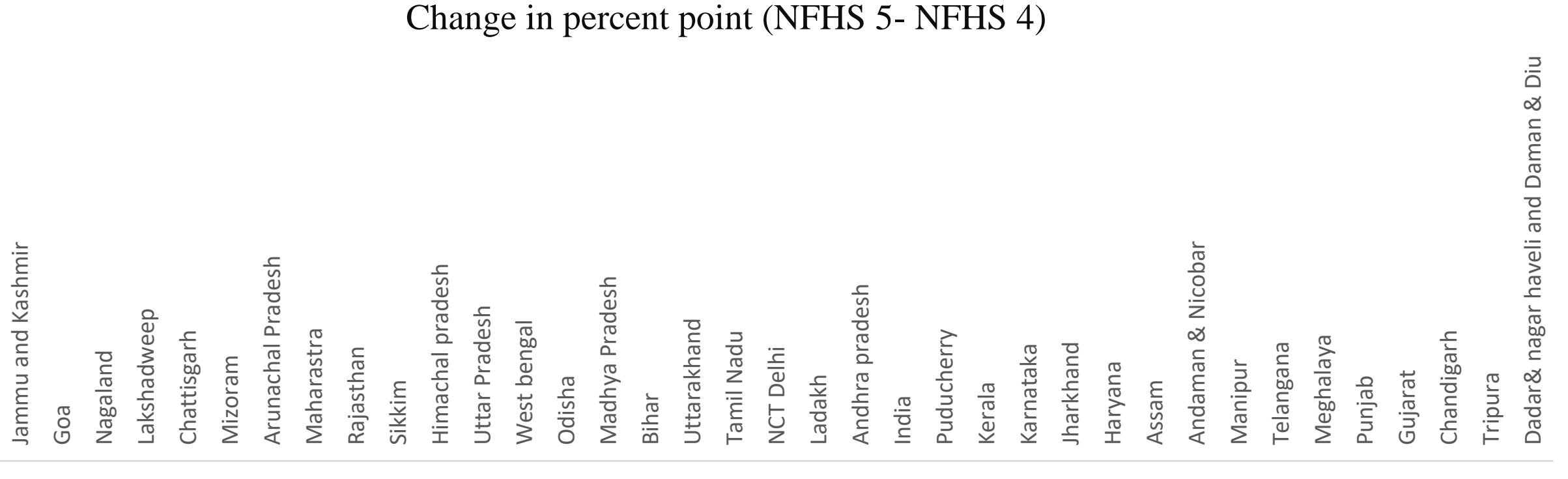

。 5 
Women age 20-24 years married before age 18 years

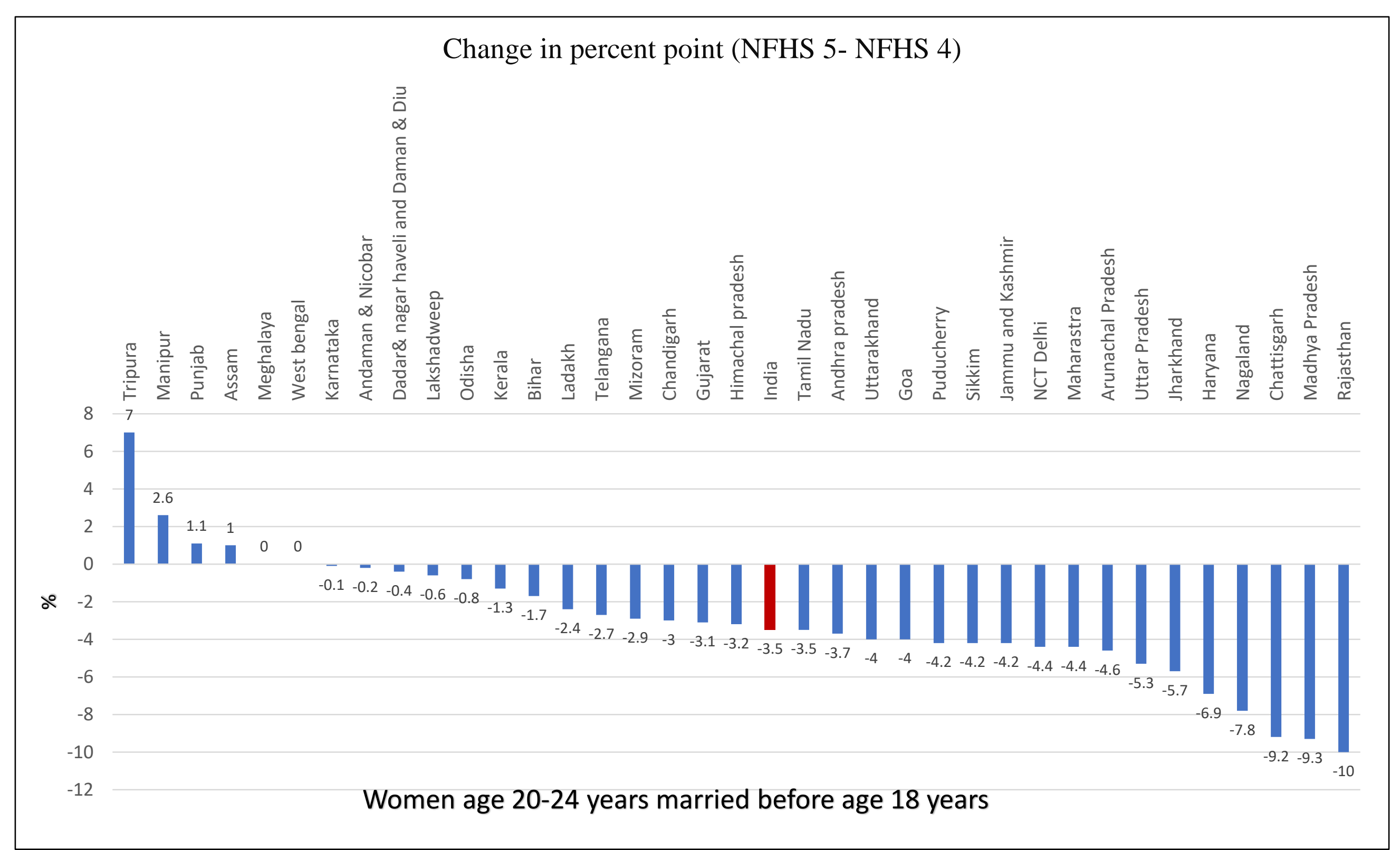




\section{NFHS 4 (2005-06) and NFHS 5 (2019-21) comparison graphs}


Neonatal mortality rate

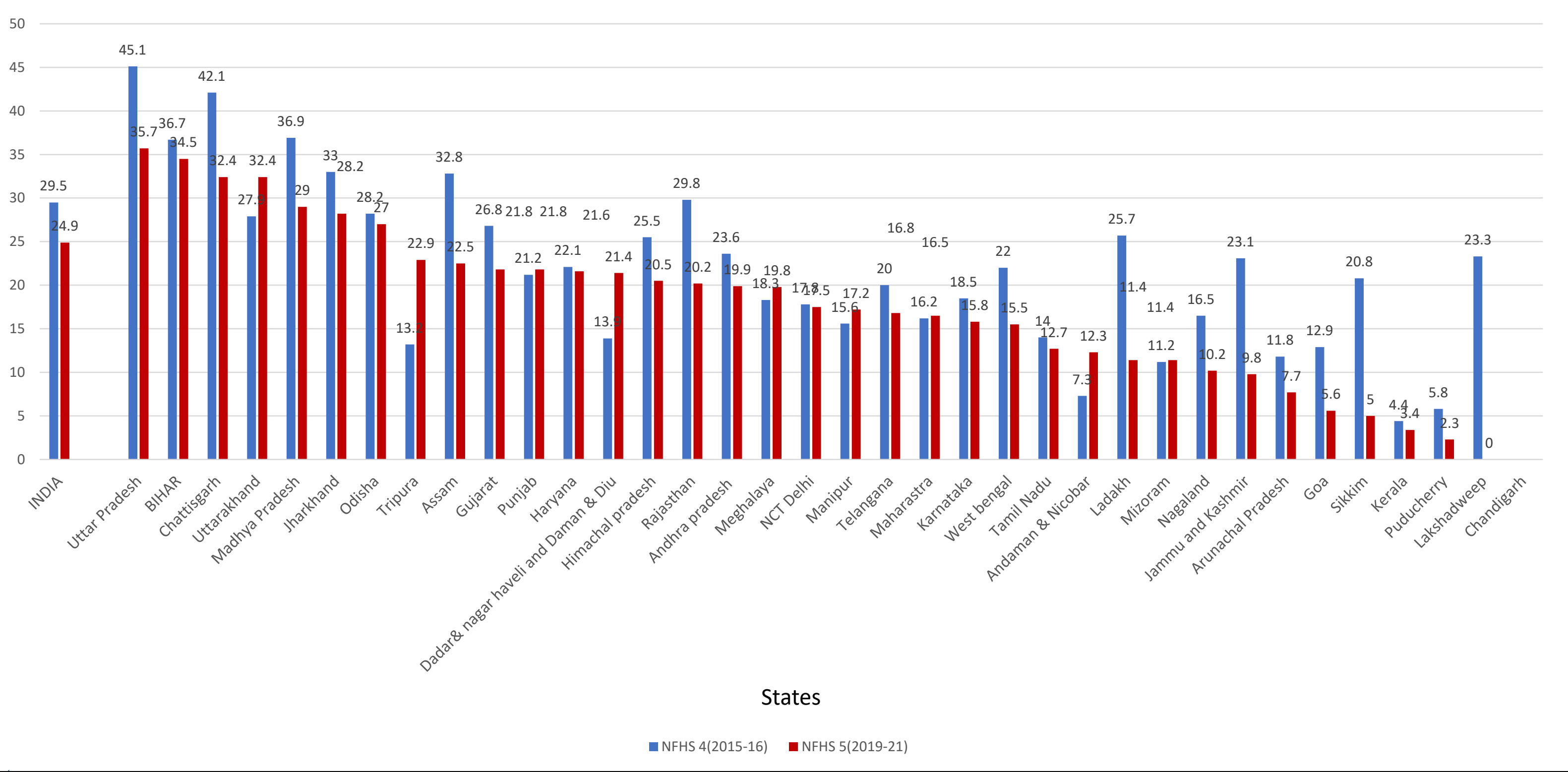


Infant mortality rate

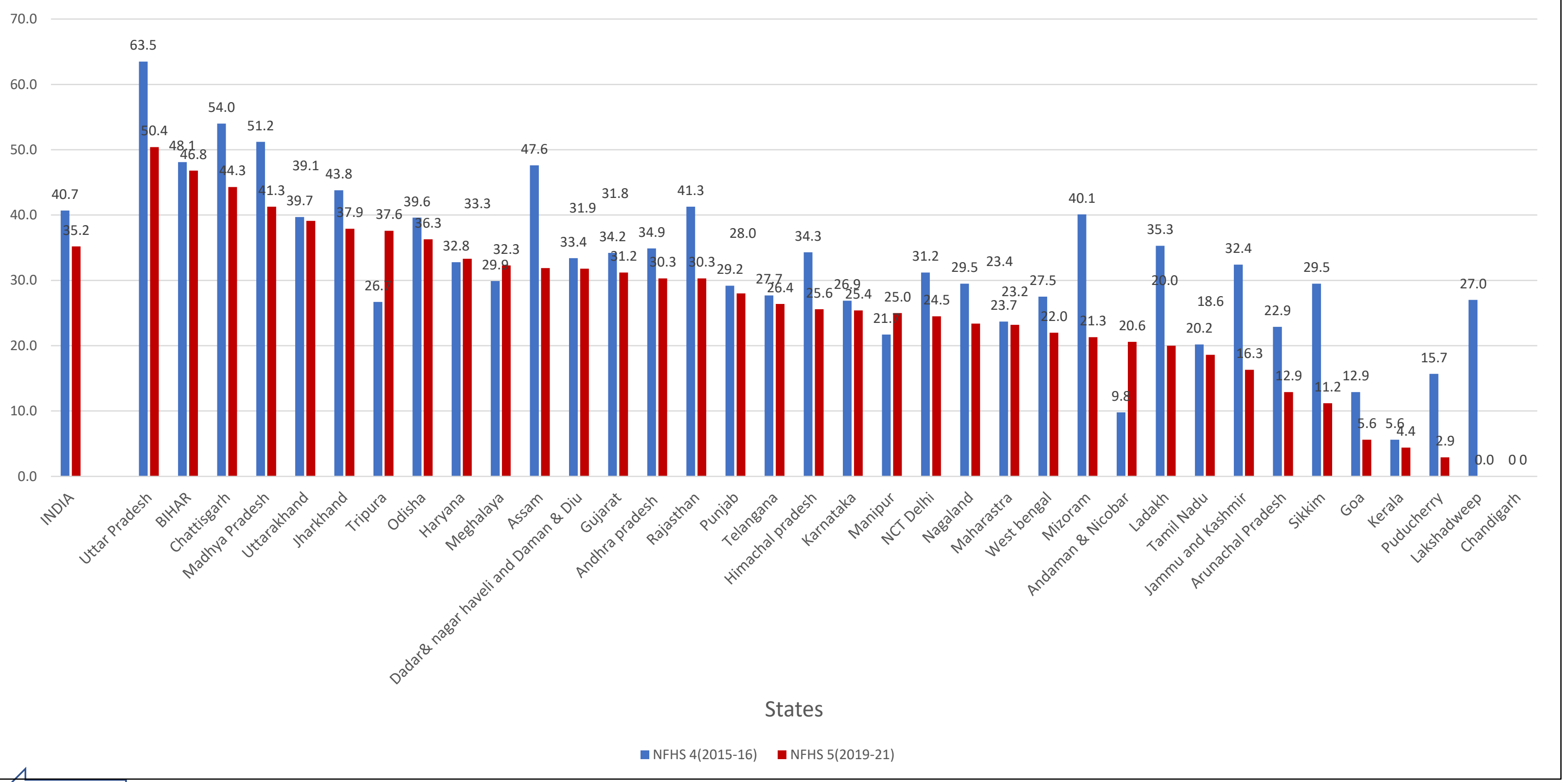




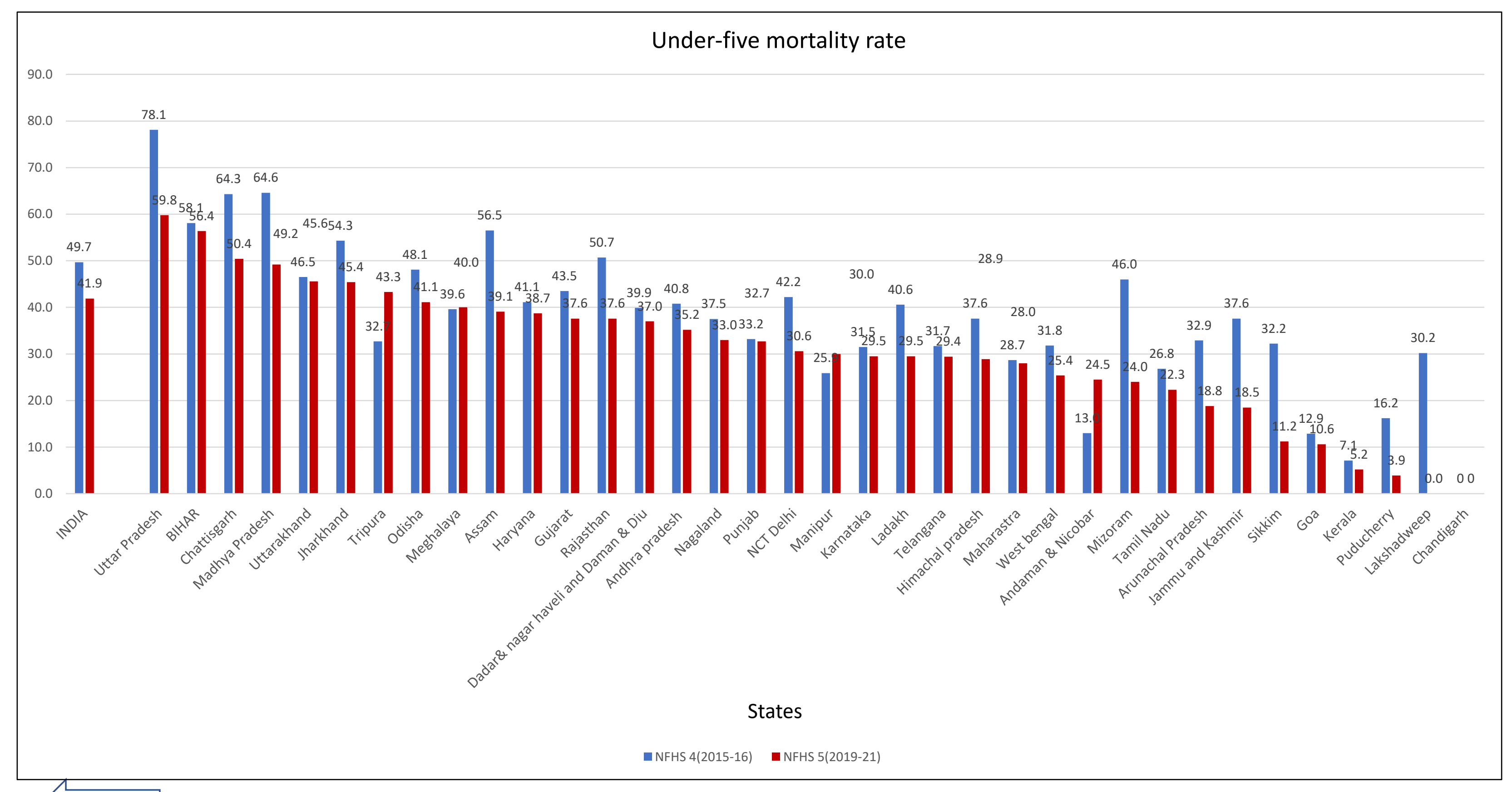

BACK 
Children under-five who are stunted (height-for-age)

60.0

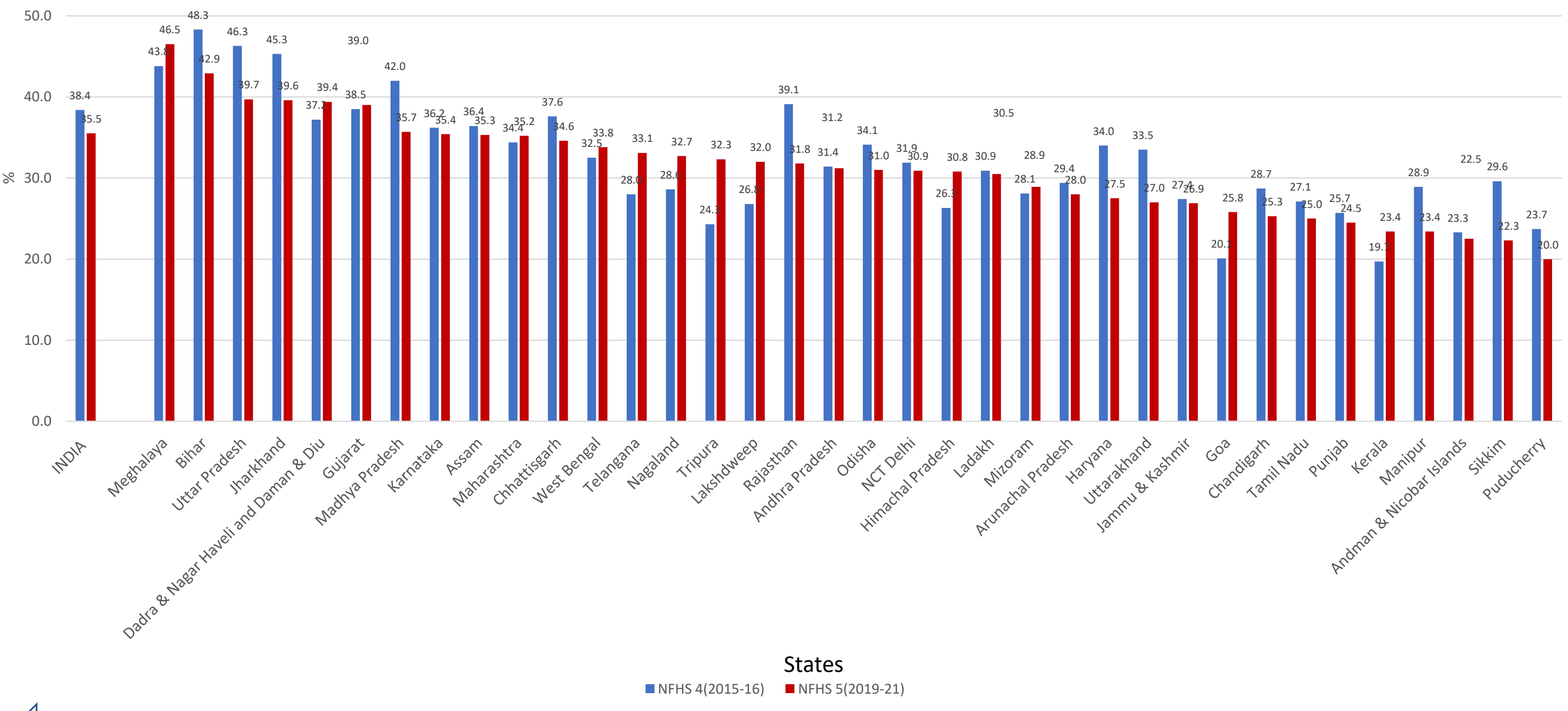


Children under 5 who are wasted(weight-for-height)

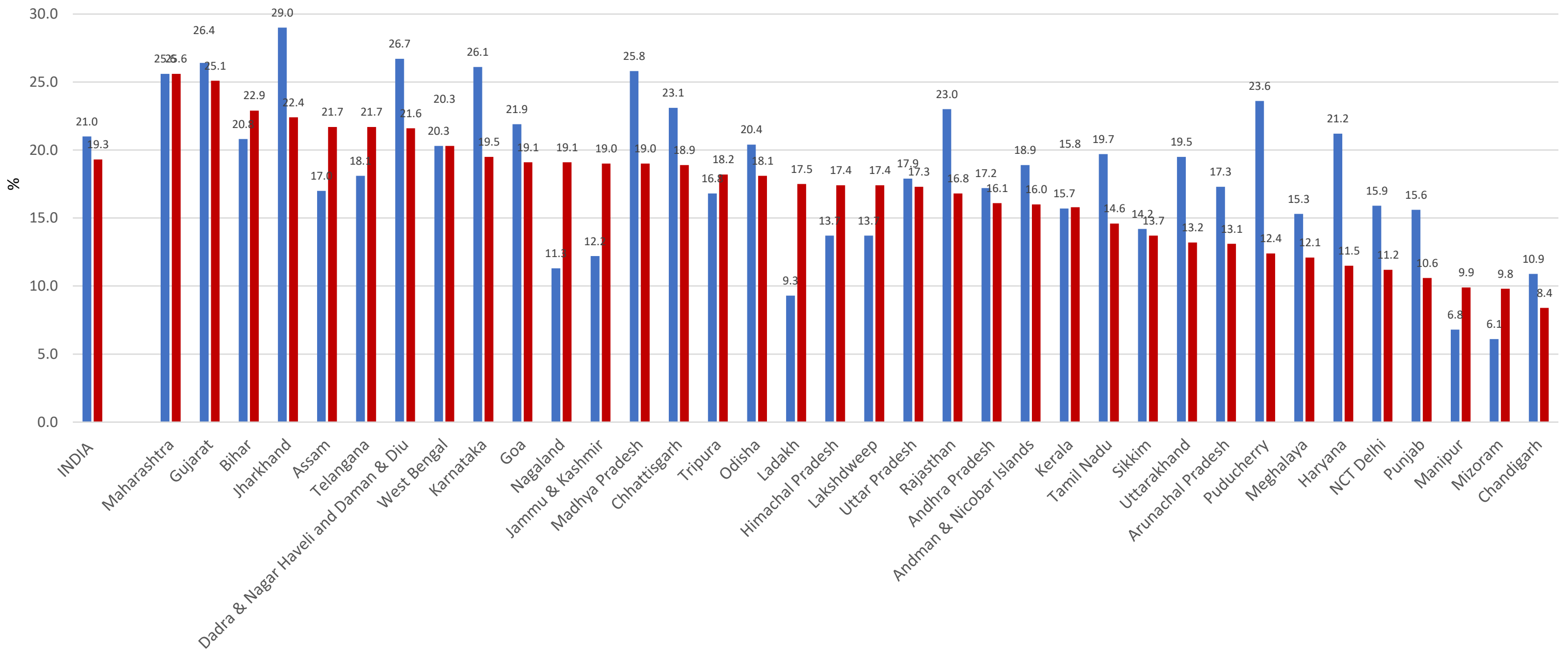

States 


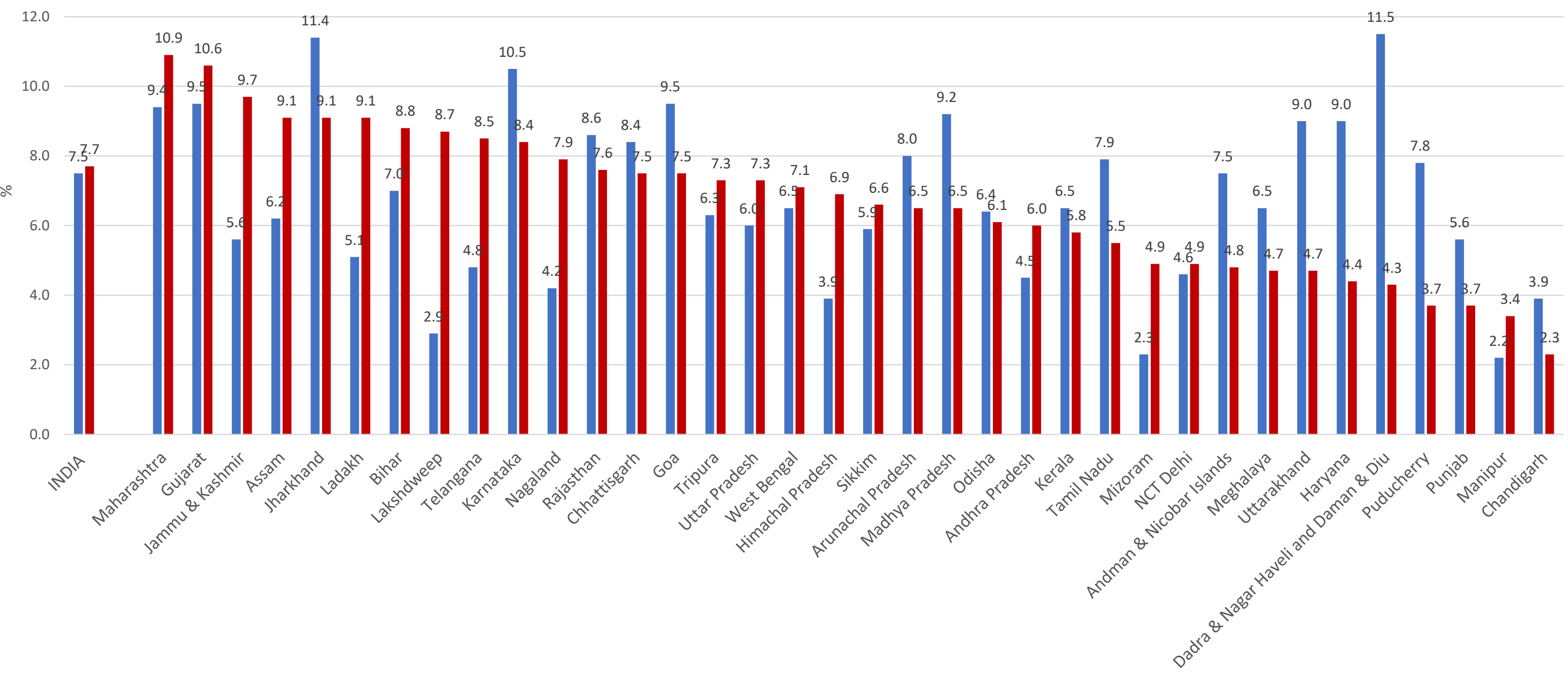

States 


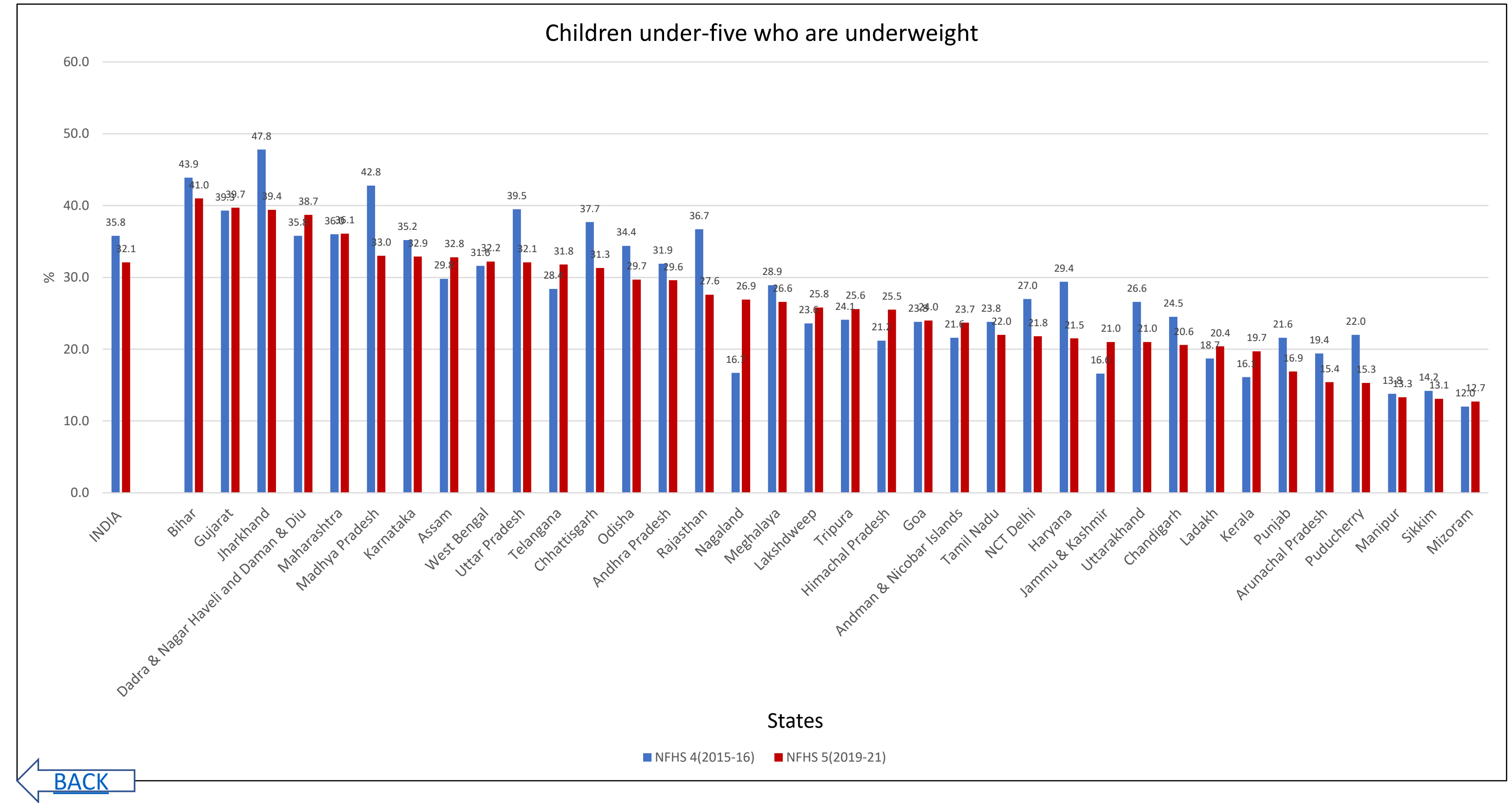




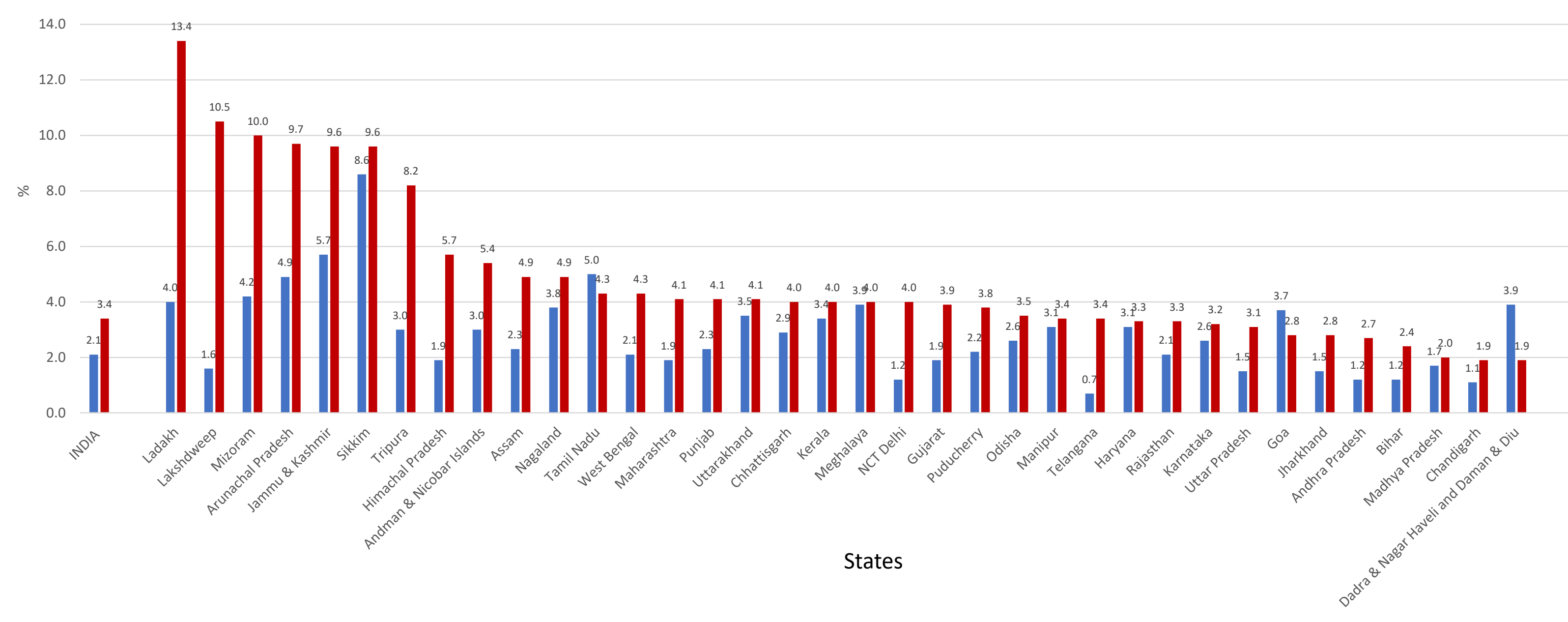


Women whose Body Mass Index (BMI) is below normal (BMI $<18.5 \mathrm{~kg} / \mathrm{m} 2)$

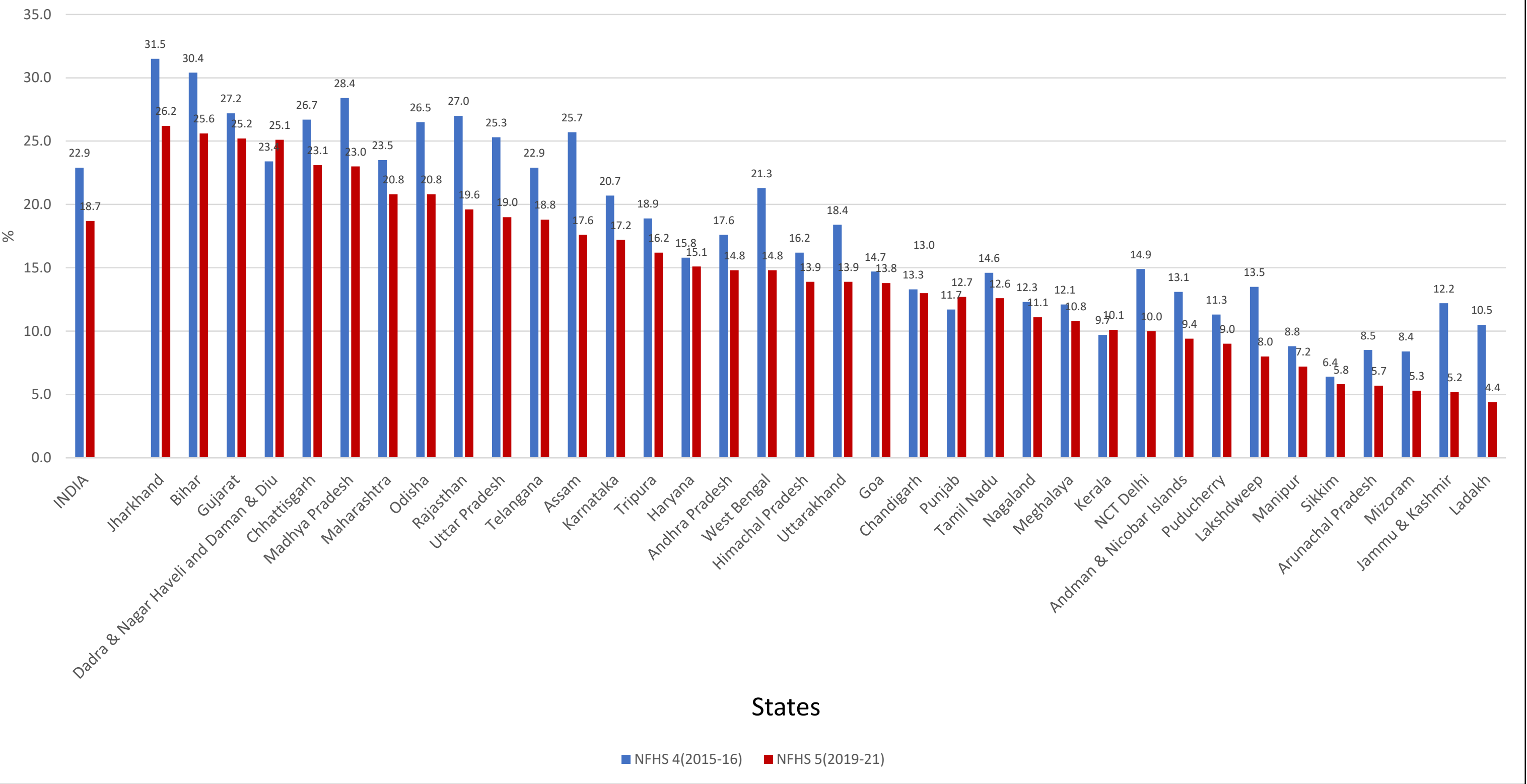


Men whose Body Mass Index (BMI) is below normal (BMI $<18.5 \mathrm{~kg} / \mathrm{m} 2$ )

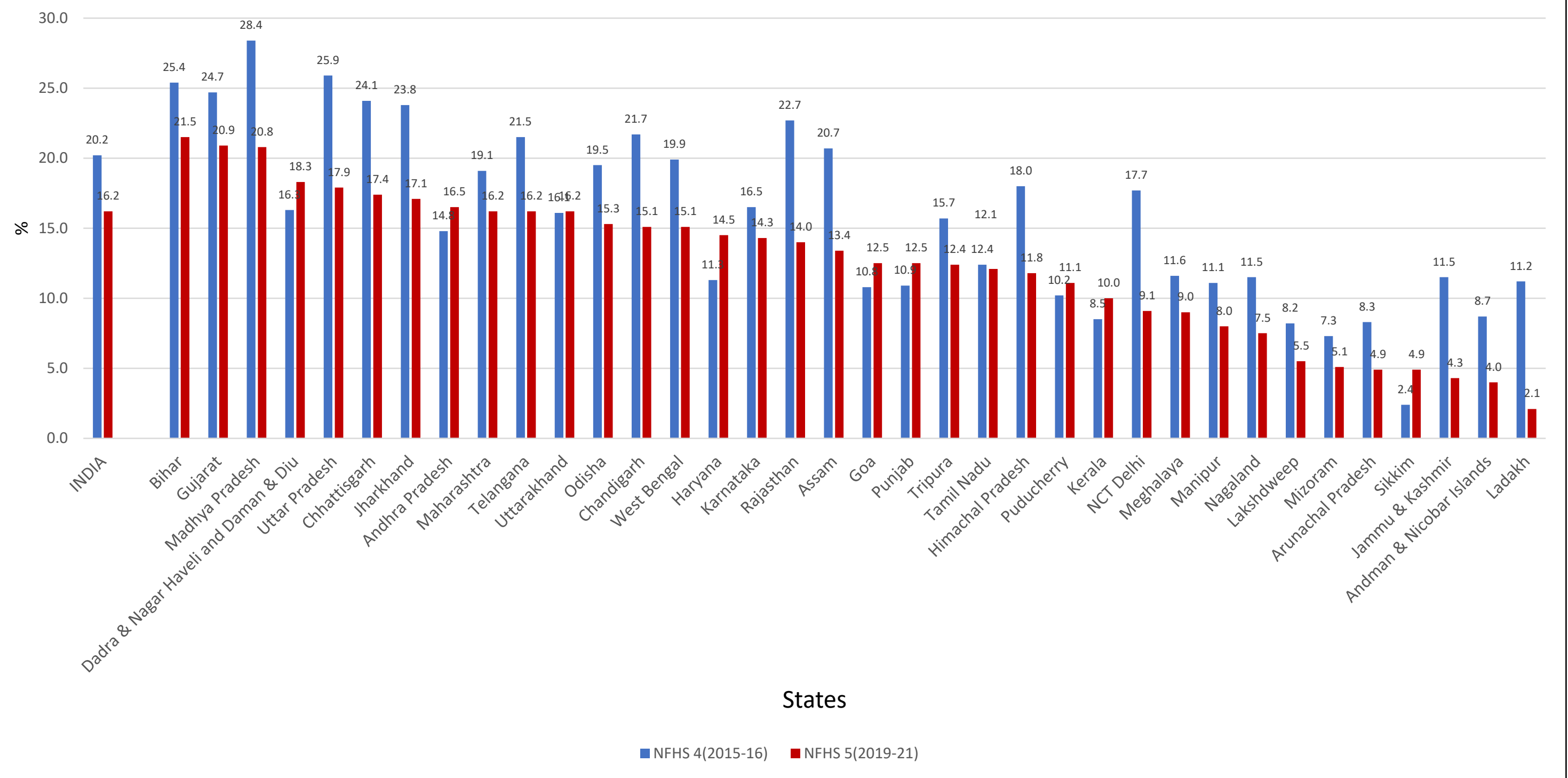


Women who are overweight or obese (BMI $\geq 25.0 \mathrm{~kg} / \mathrm{m} 2)$

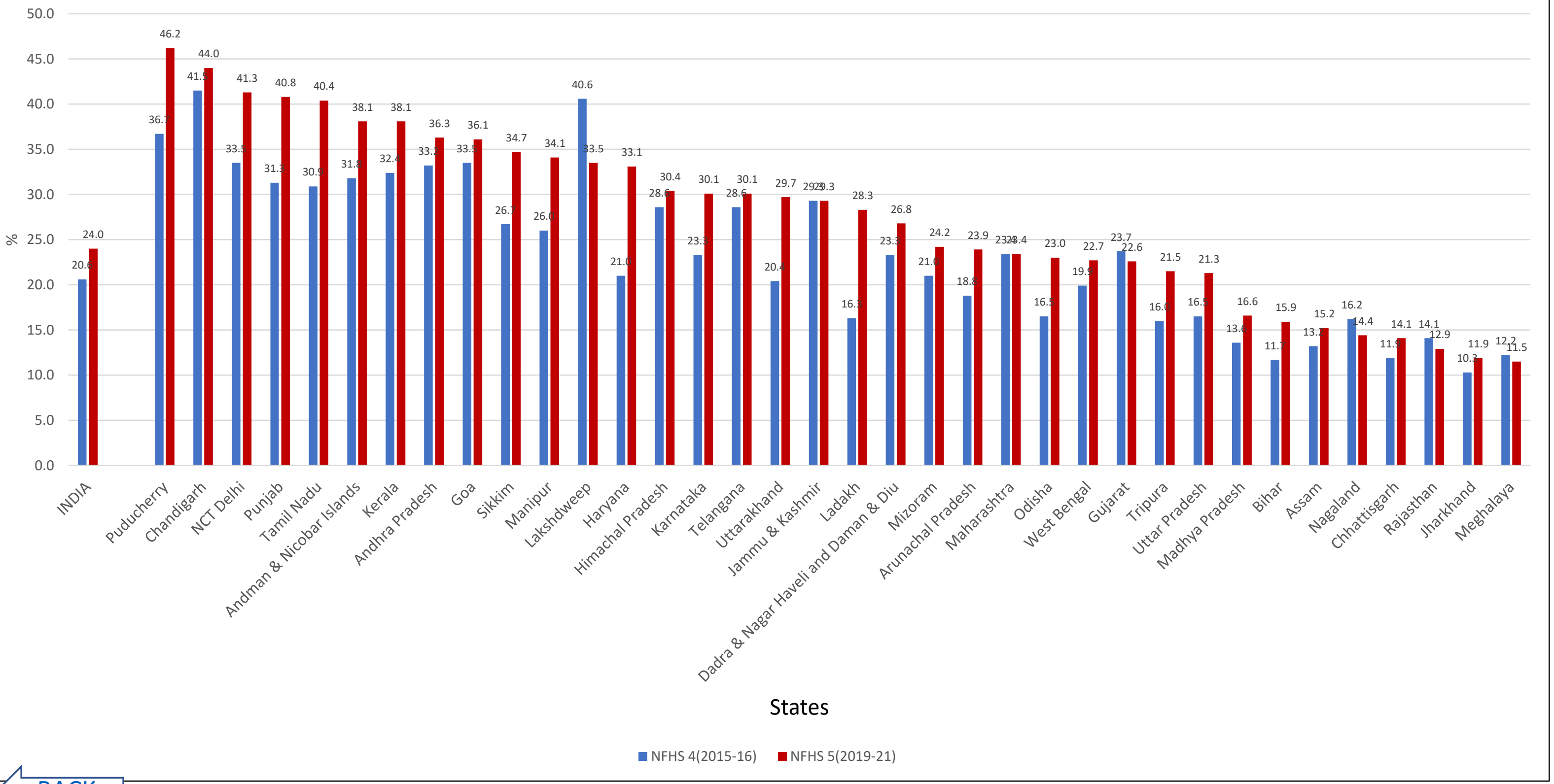


Men who are overweight or obese $(\mathrm{BMI} \geq 25.0 \mathrm{~kg} / \mathrm{m} 2$

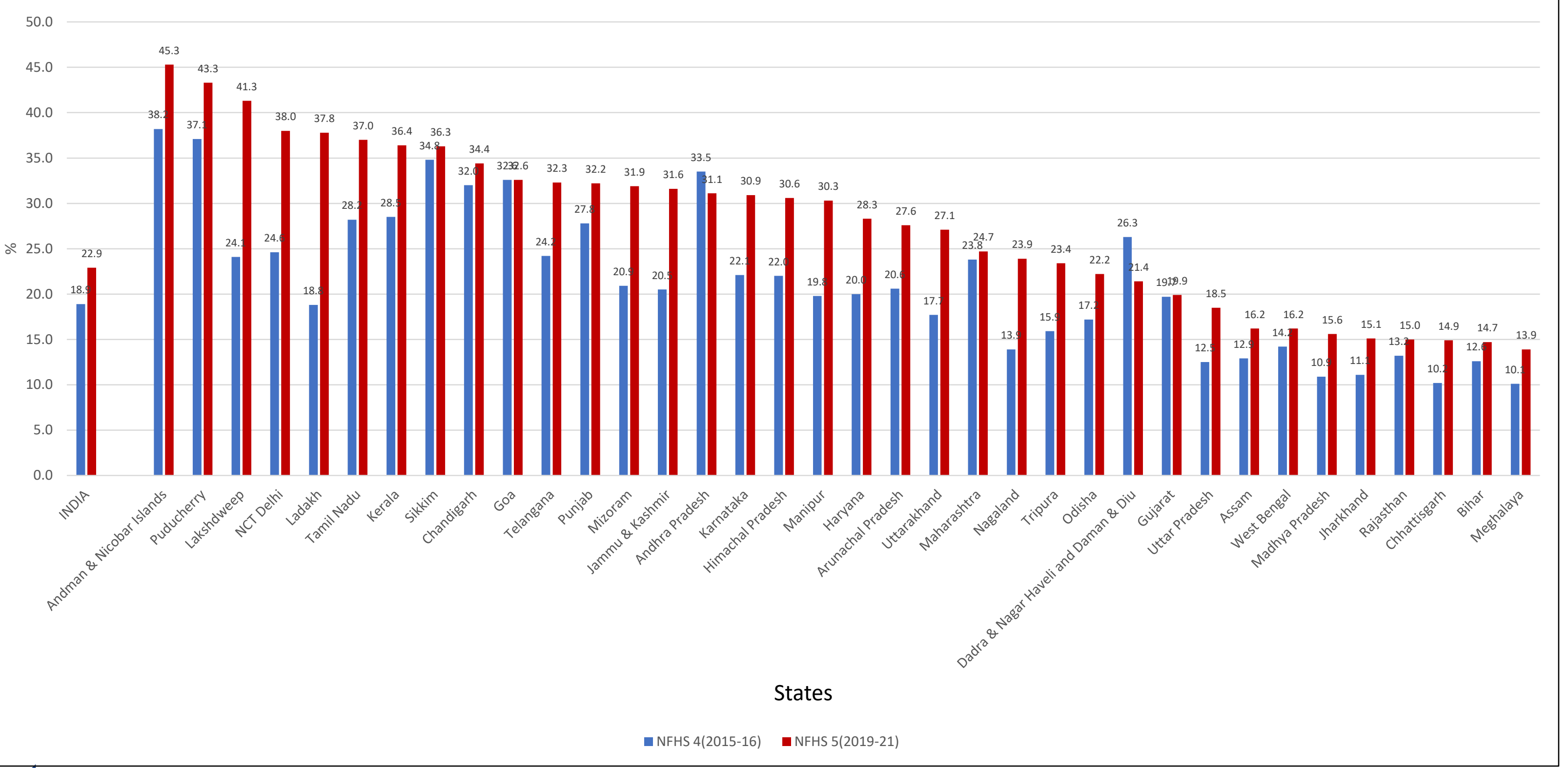




\section{Children age 6-59 months who are anaemic $(<11.0 \mathrm{~g} / \mathrm{dl})$}

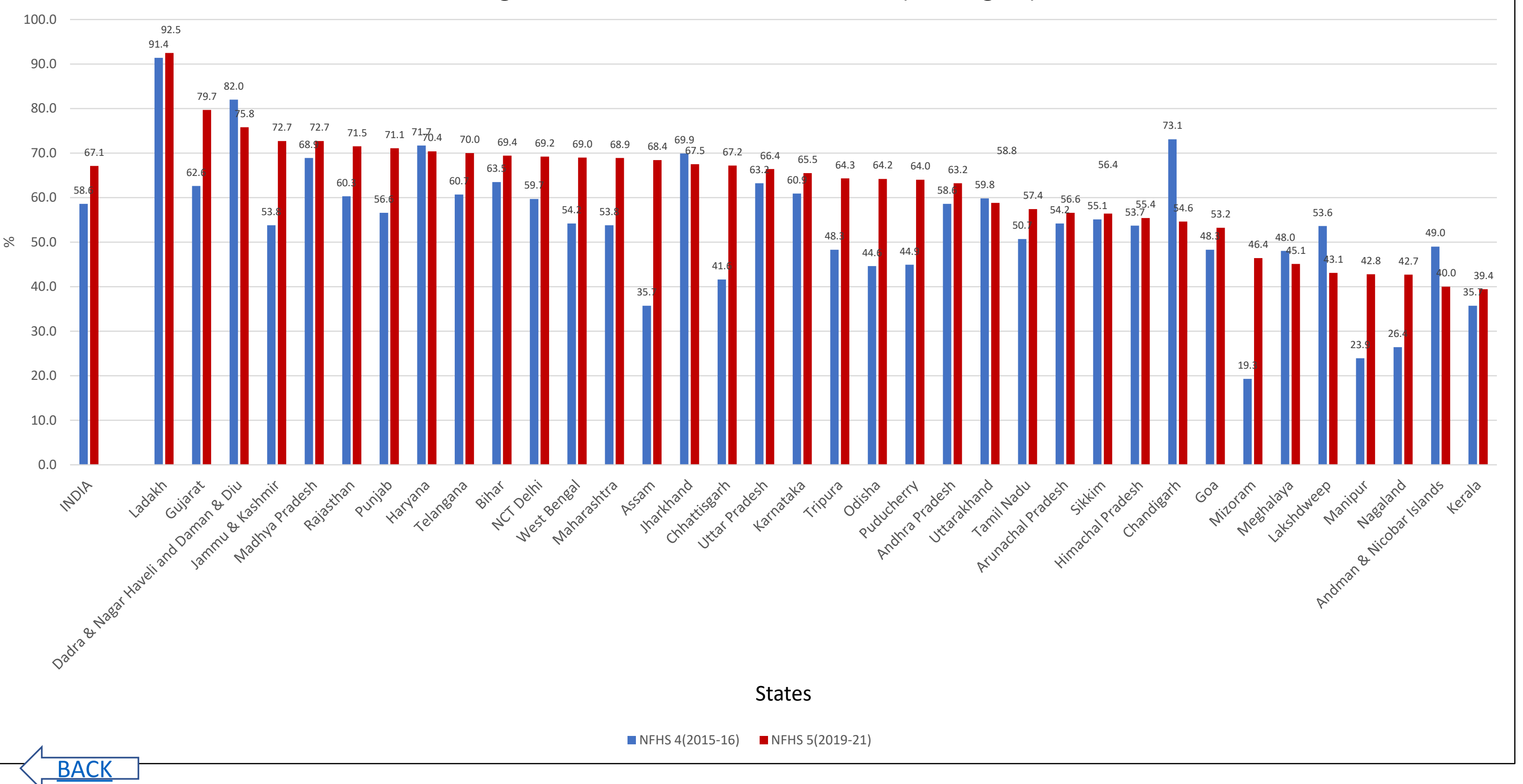


Adolescent girls, age $15-19$ years who are anaemic (\%)

120.0

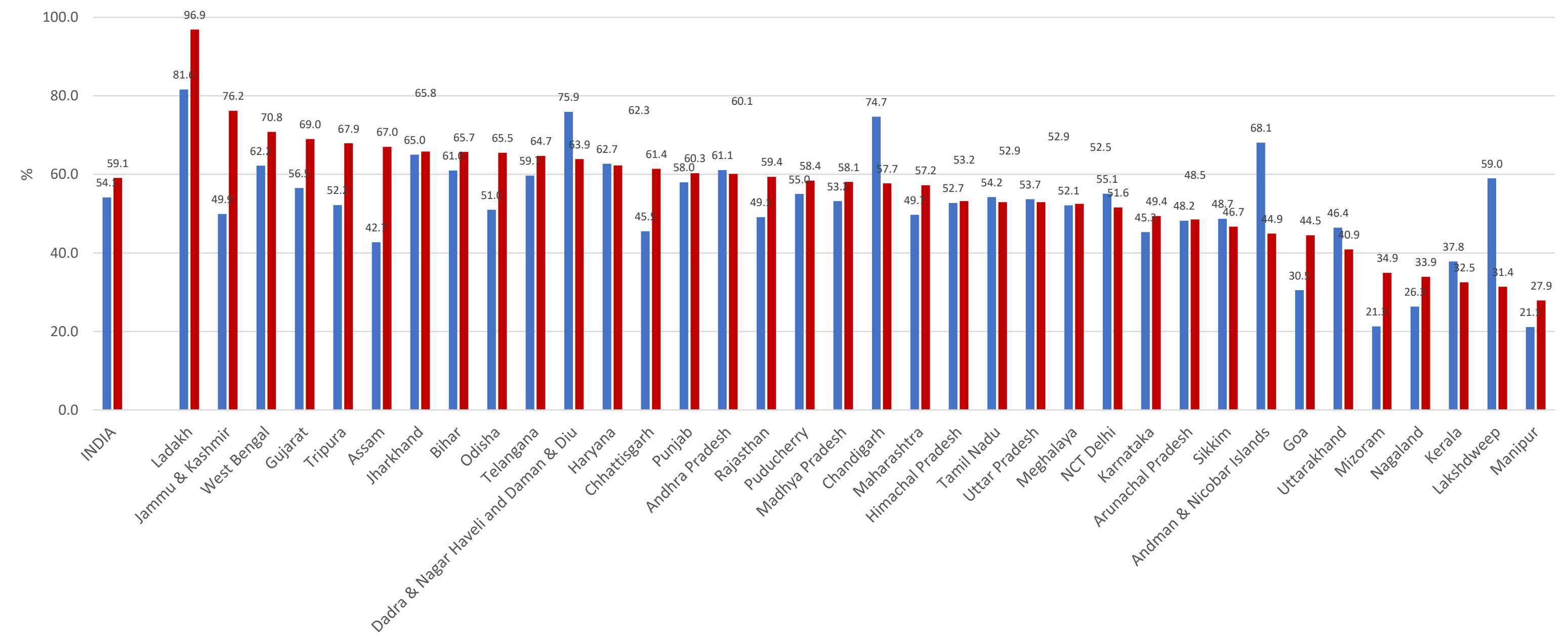

States

- NFHS 4(2015-16) NFHS 5(2019-21) 


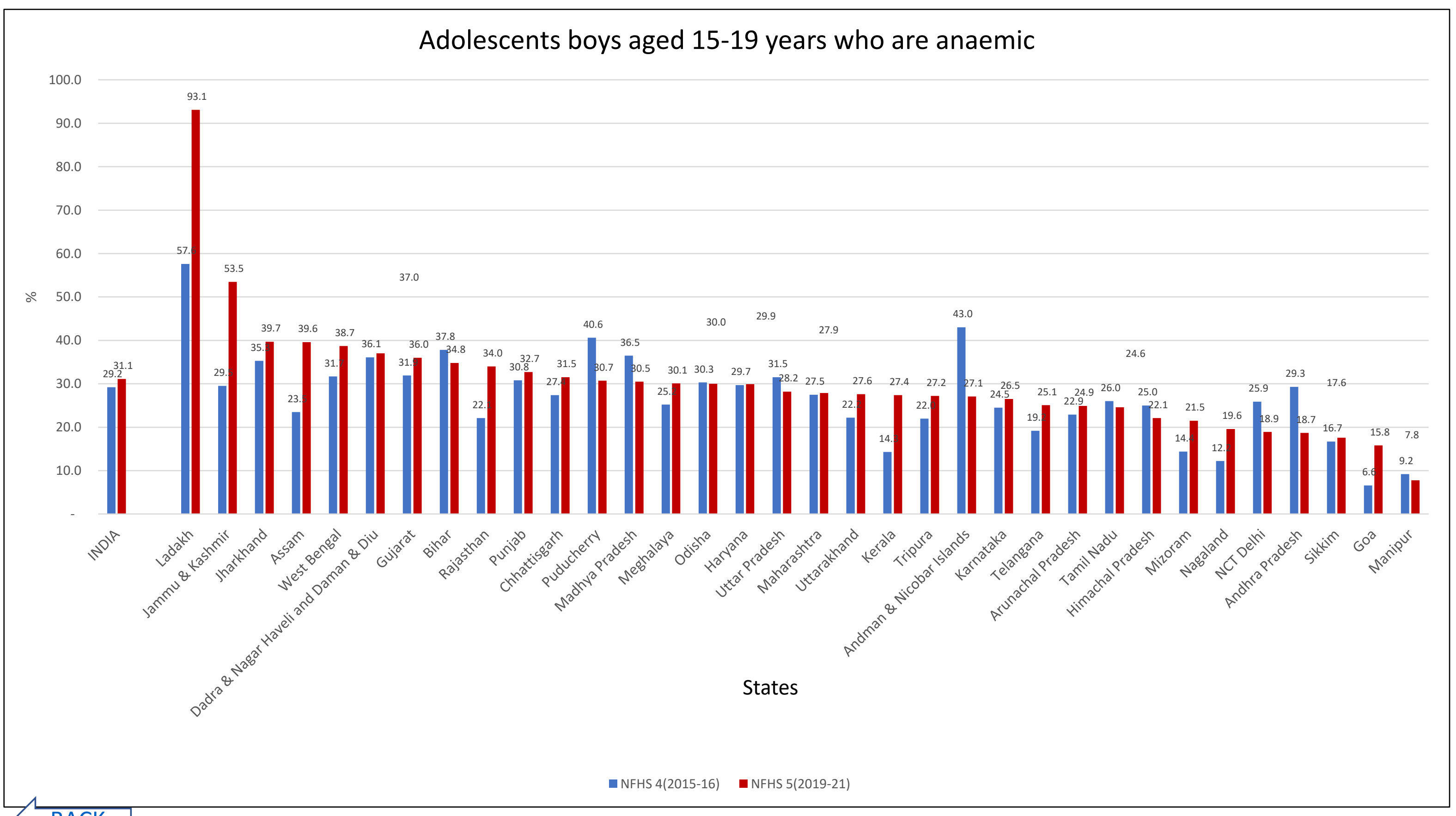


Pregnant women aged $15-49$ years who are anaemic

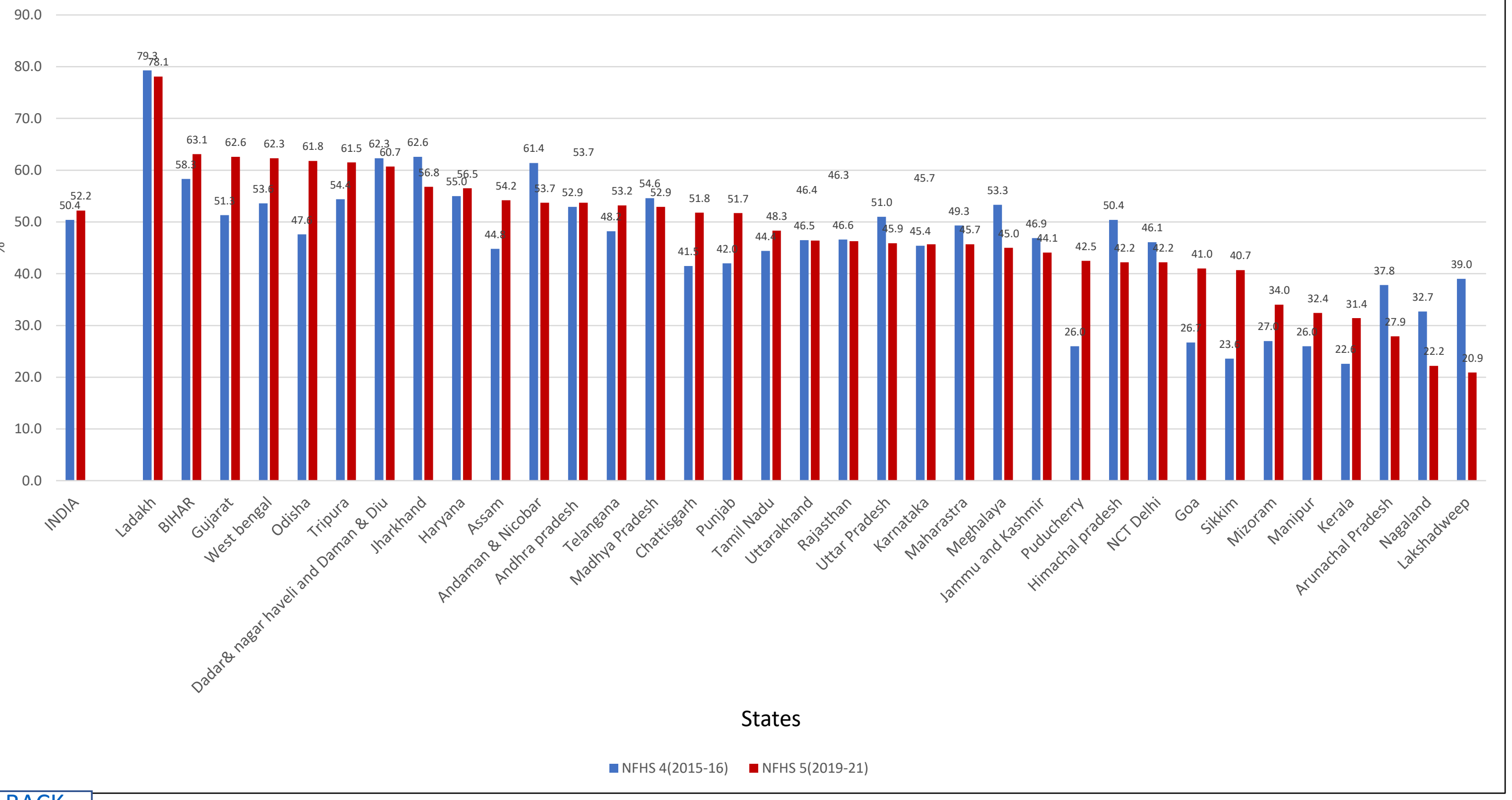




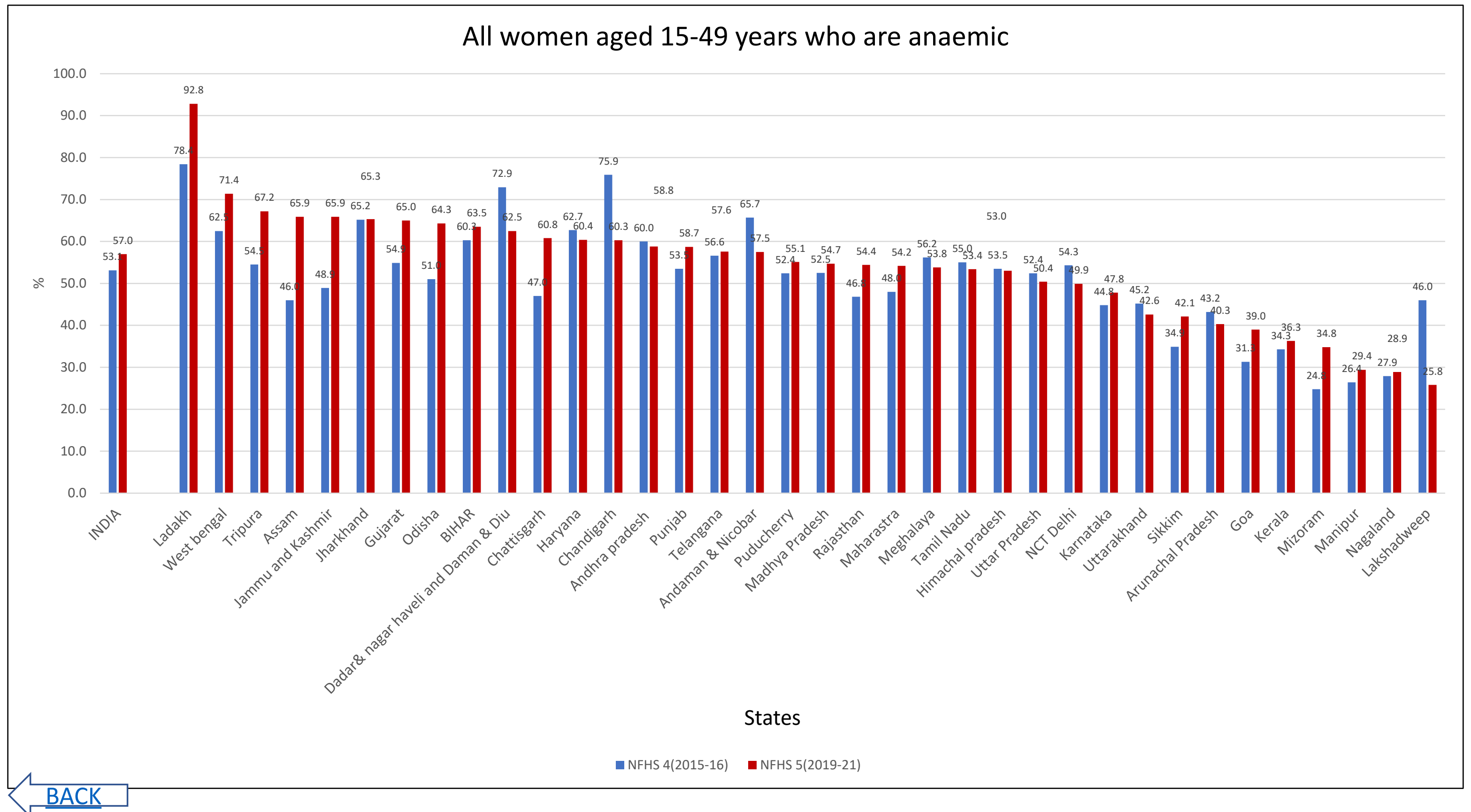


Men age $15-49$ years who are anaemic

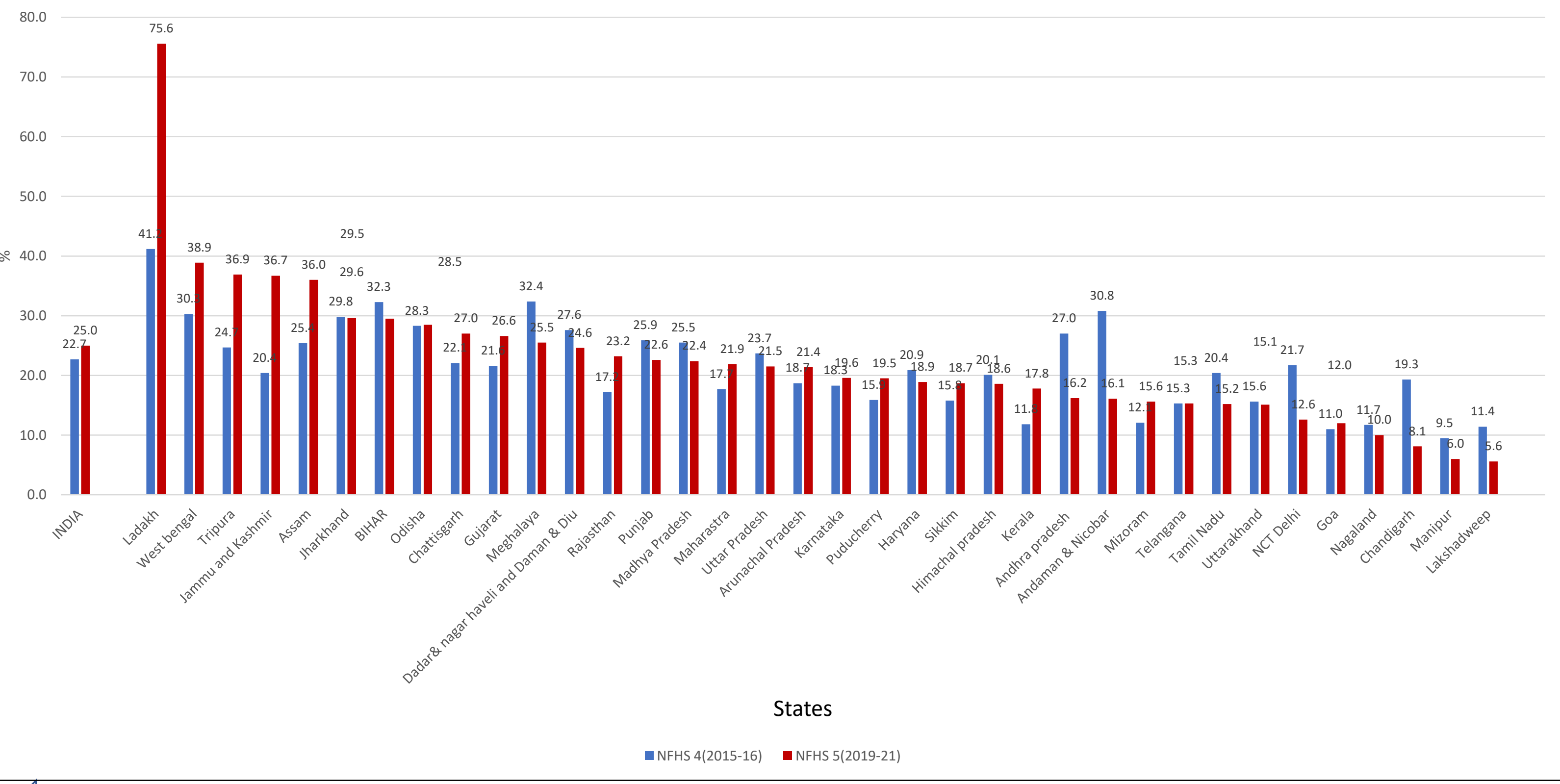




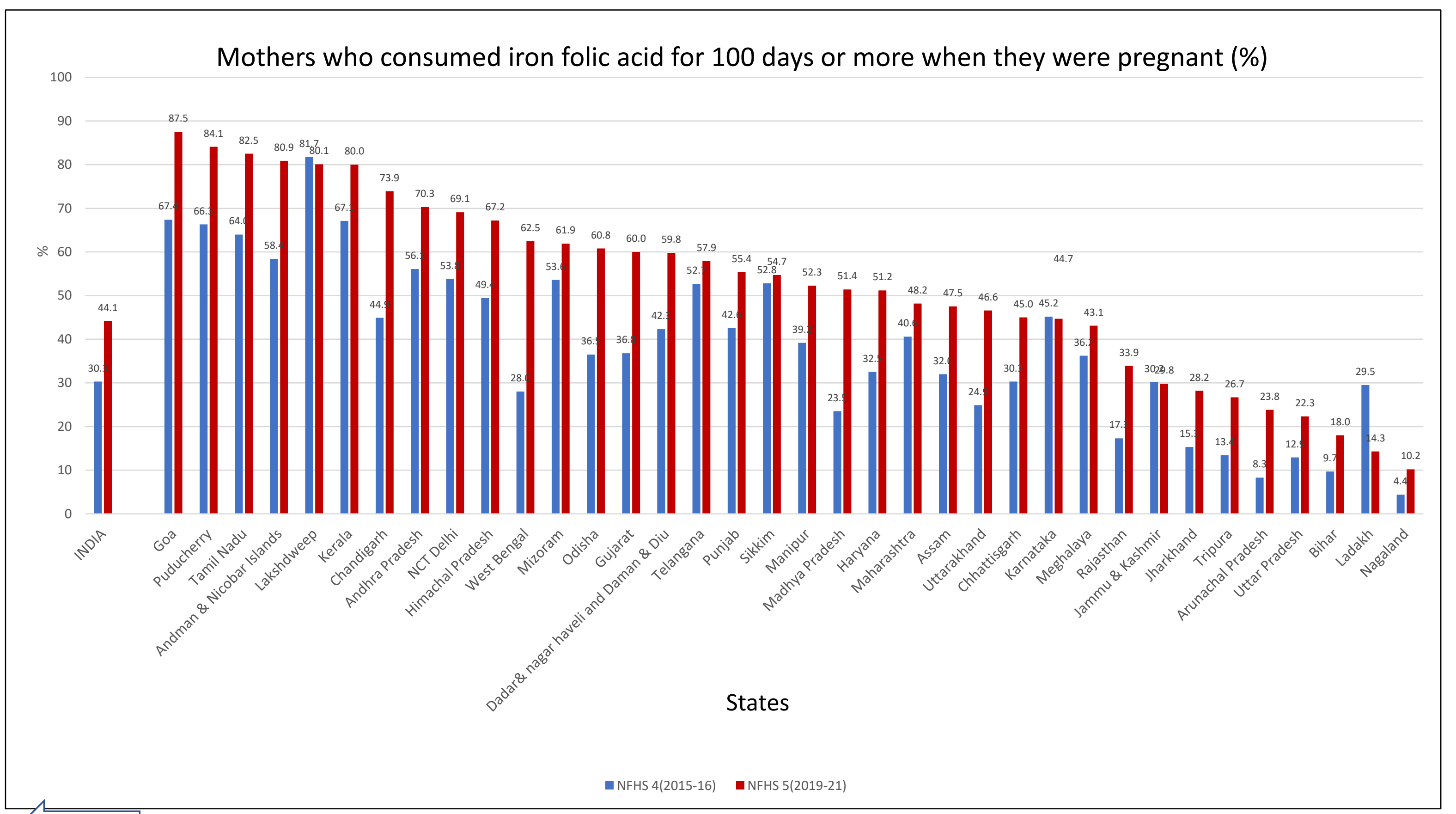


Mothers who consumed iron folic acid for 180 days or more when they were pregnant

80.0

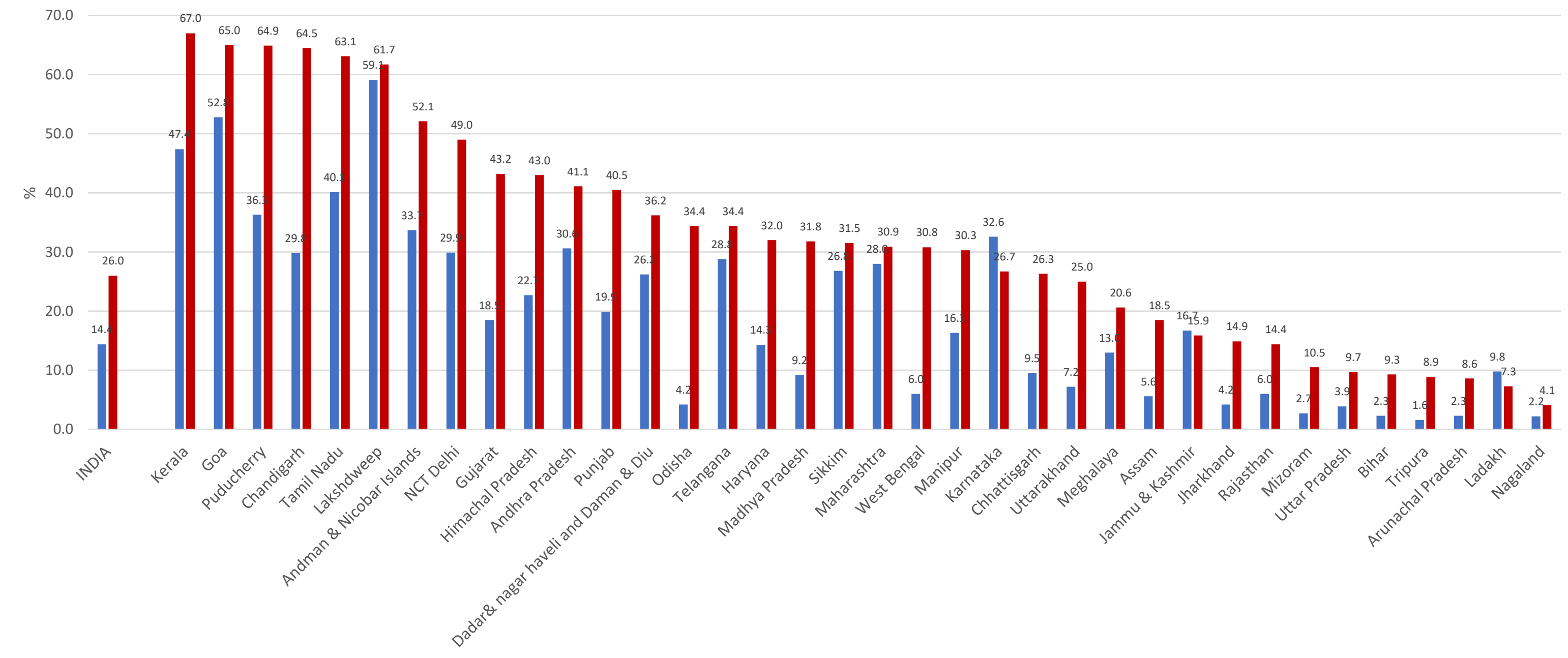

States 


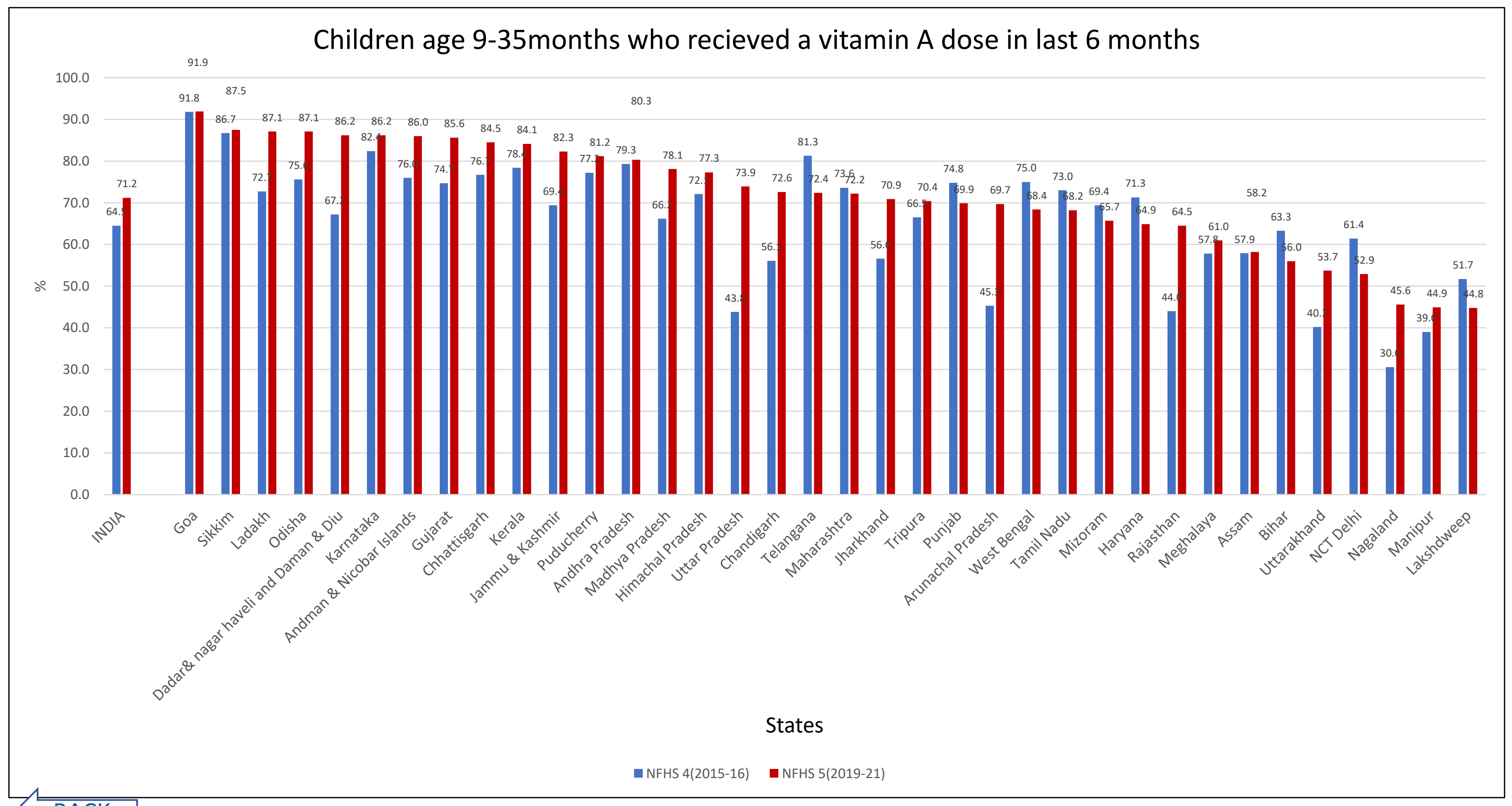




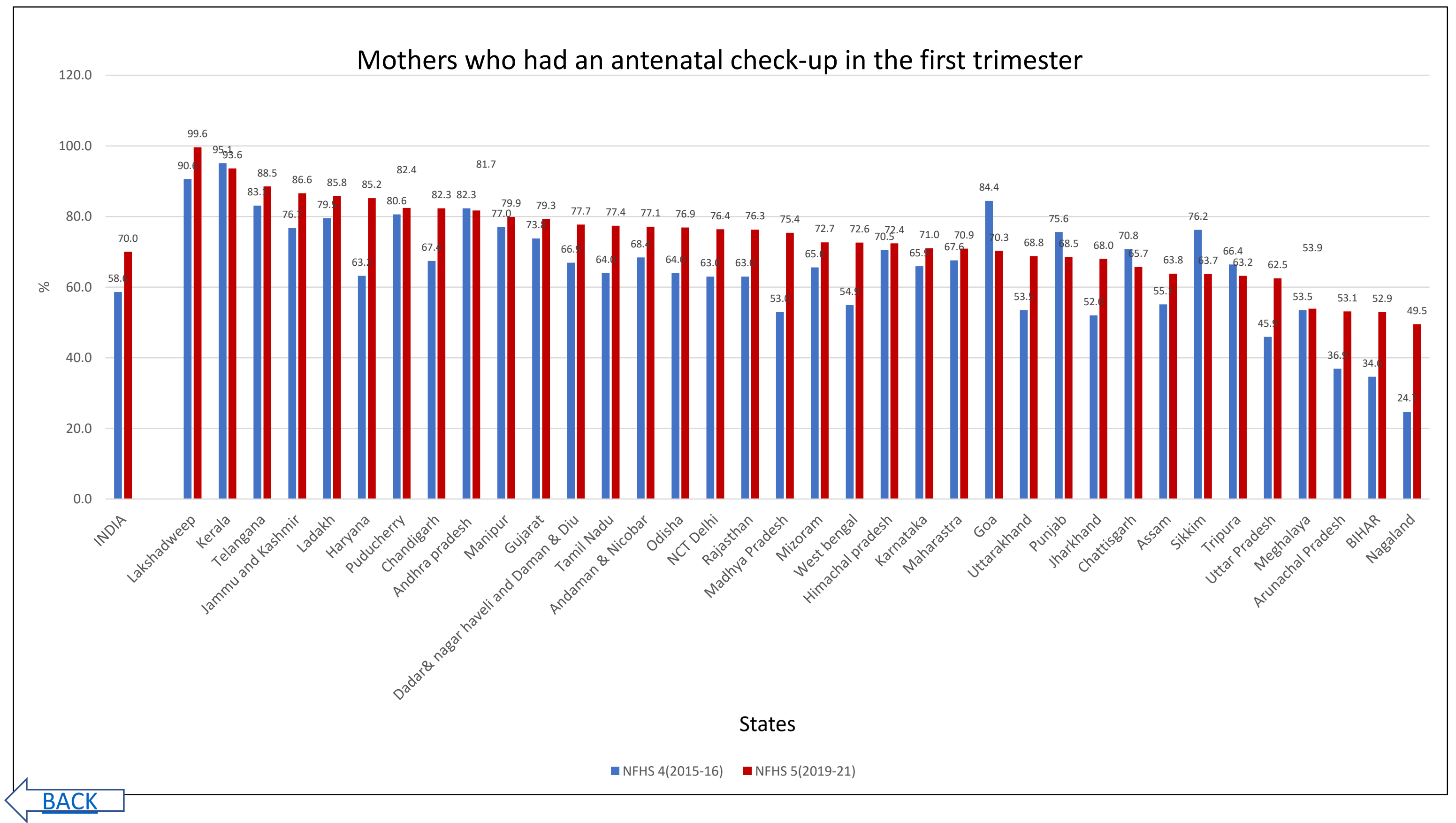


Mothers who had at least 4 antenatal care visit

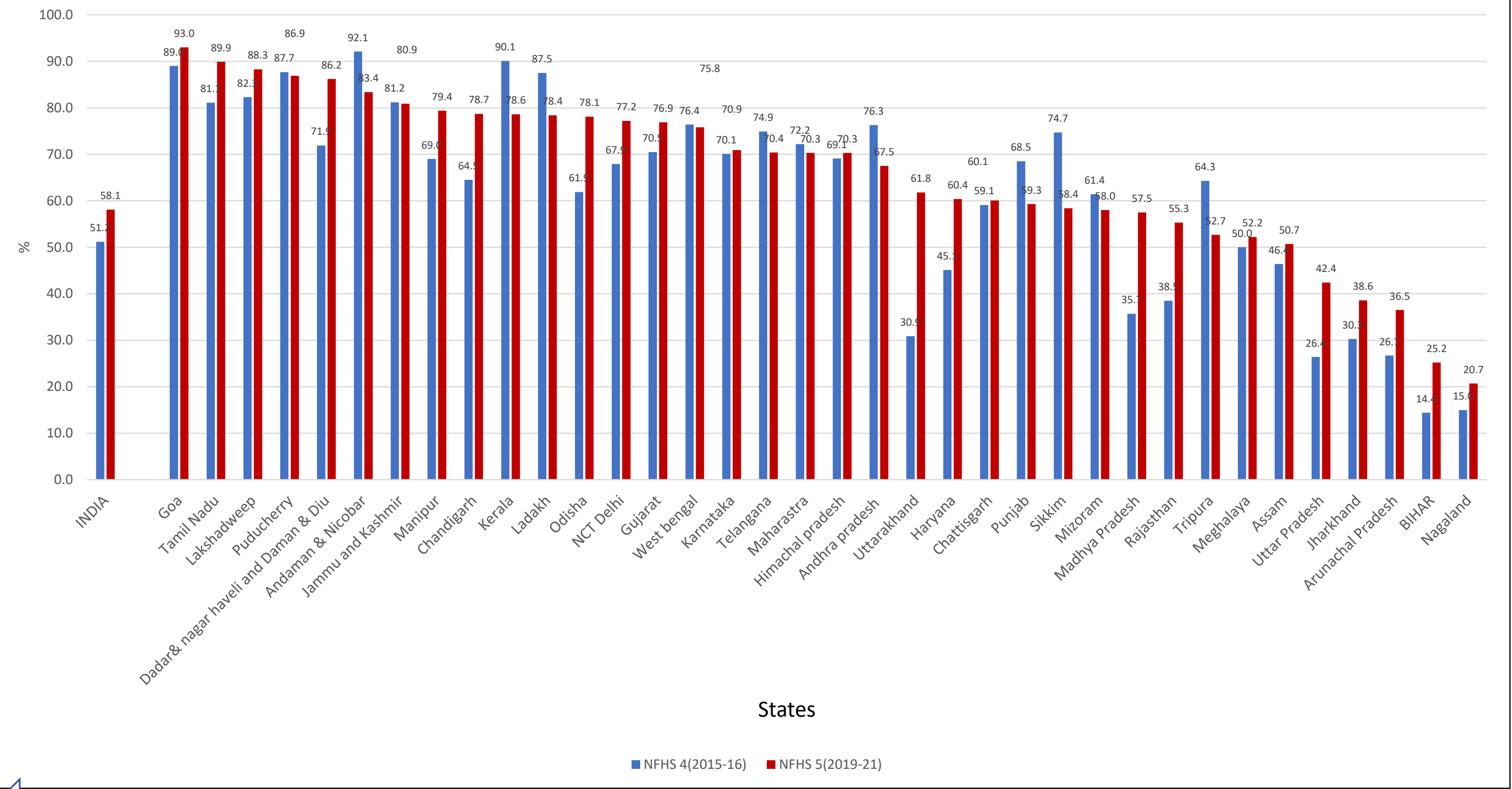


Mothers who recieved post natal care from a doctor/Nurse/LHV/ANM/midwife/other health personnel within two days of delivery

120.0 86.2

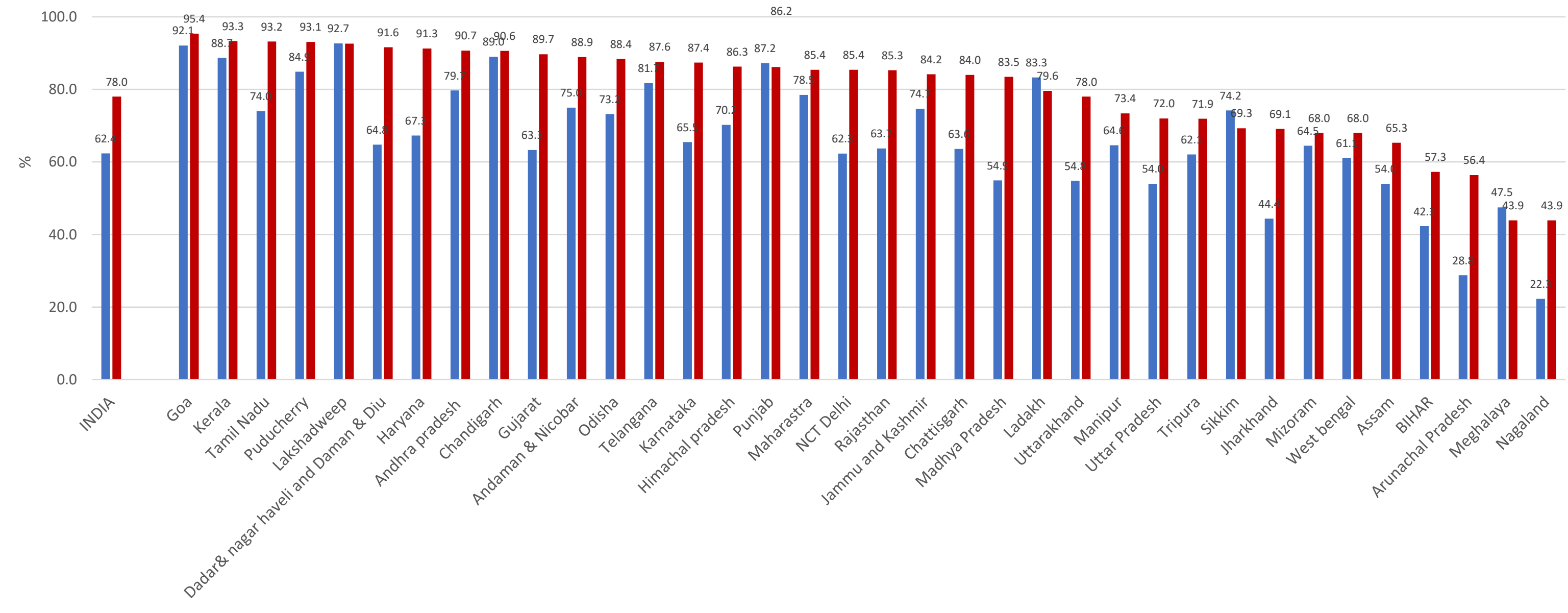

States 
Institutional births

$\begin{array}{lllll}120 & 99.8 & 99.6 & 99.6 & 99.6\end{array}$

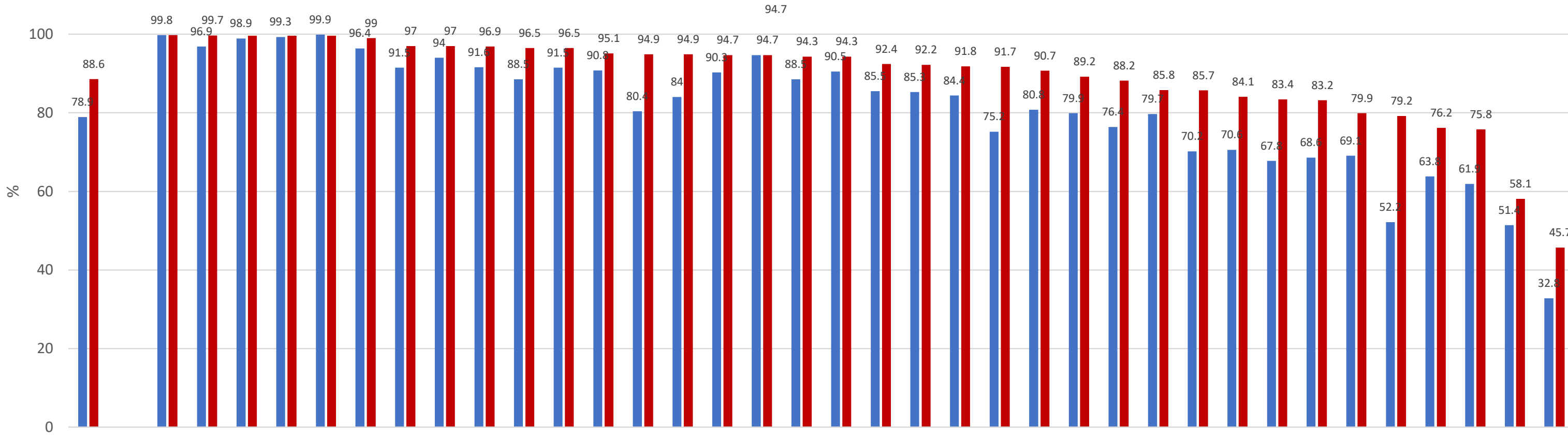

(1)

\section{States}


Births delivered by caesarean section

70.0

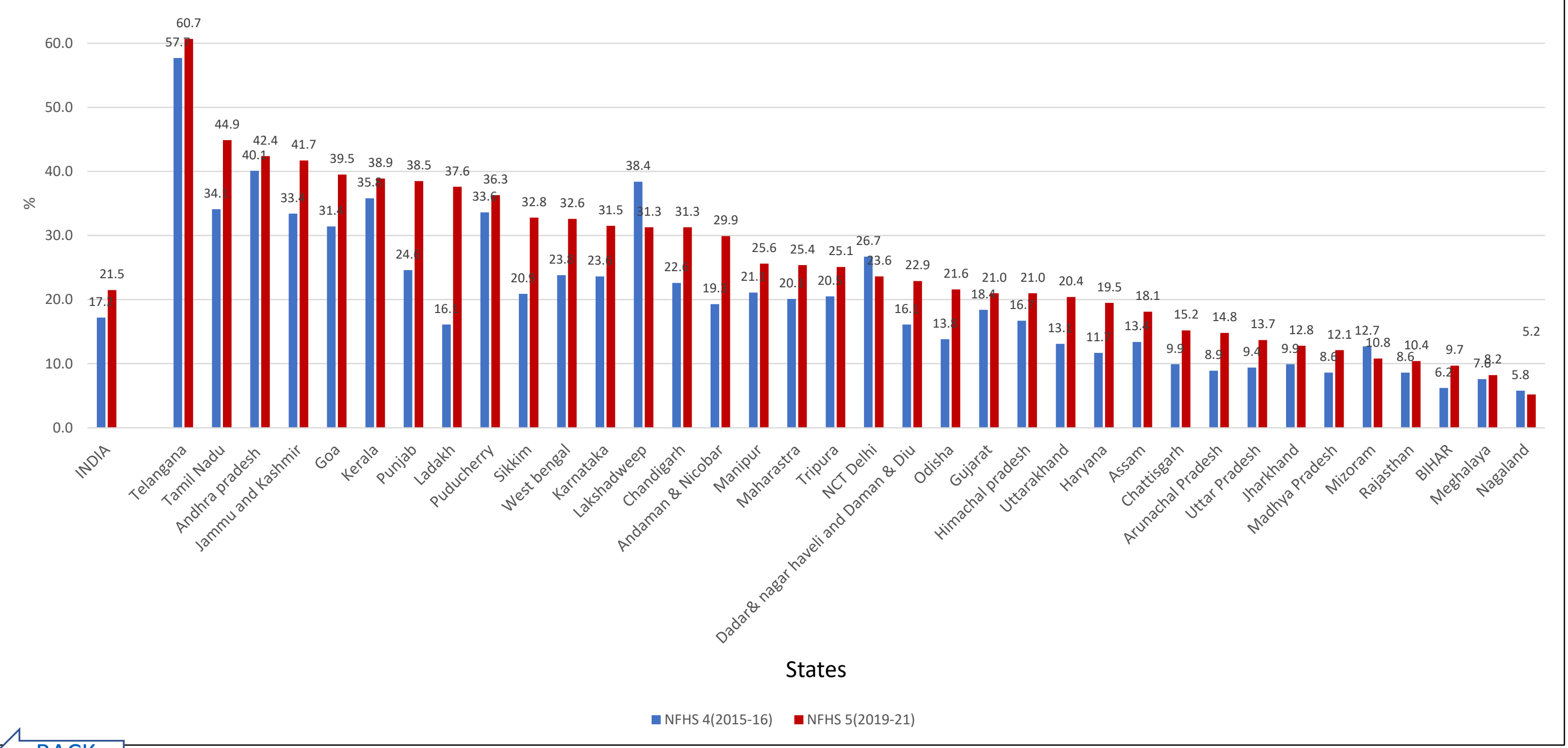




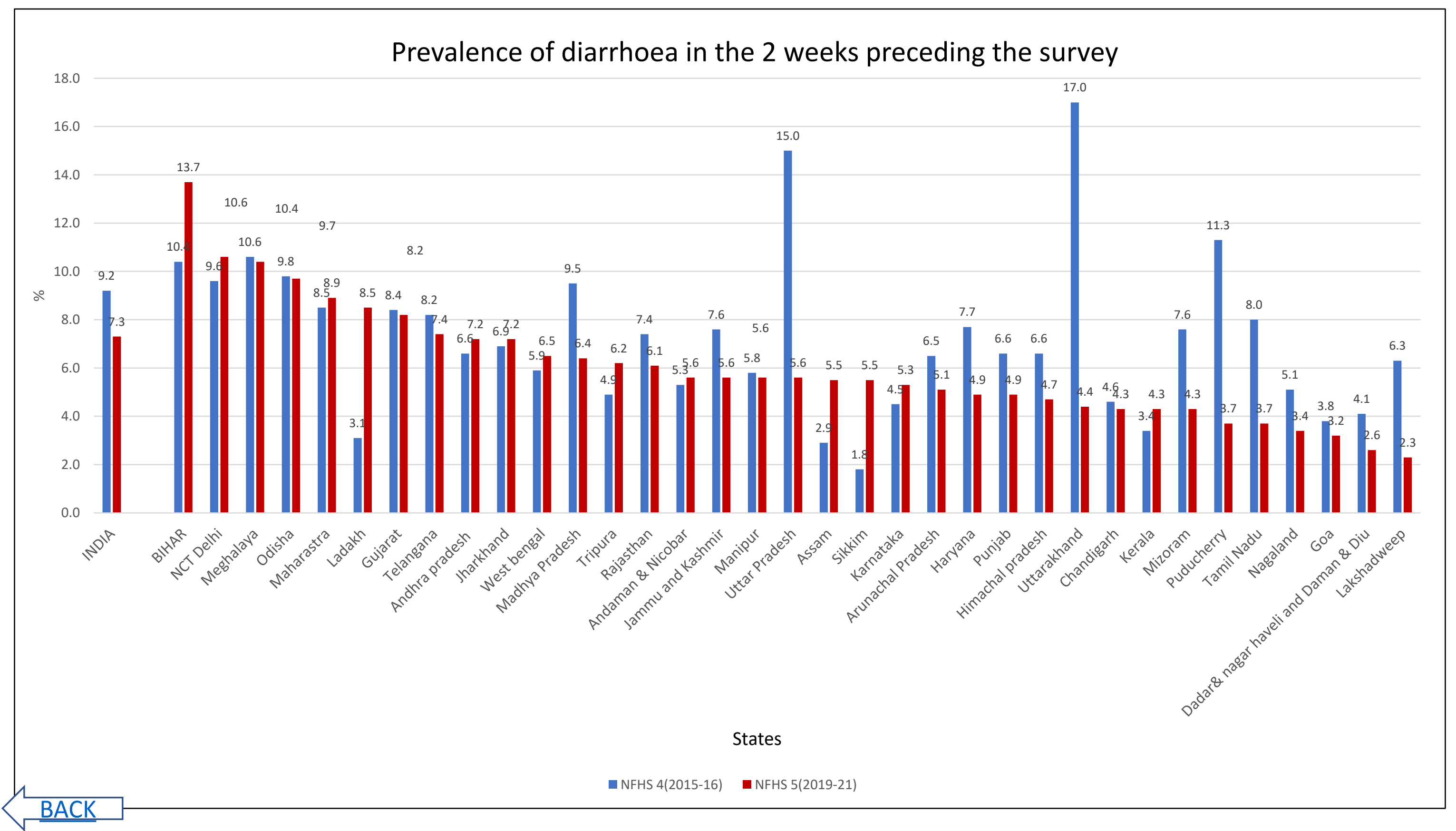


Prevalence of symptoms of acute respiratory infection (ARI) in the 2 weeks preceding the survey

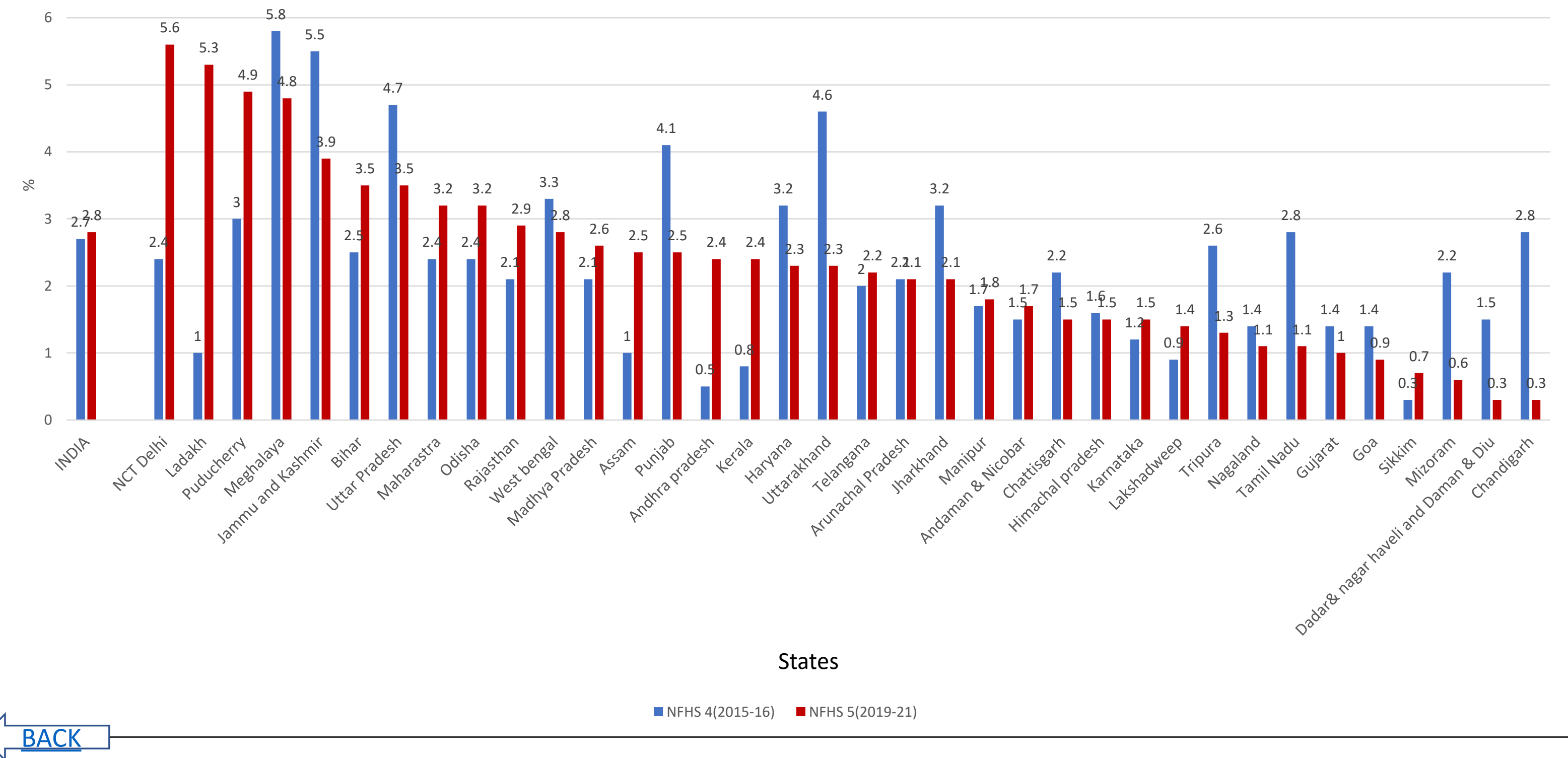


Children with diarrhoea in the 2 weeks preceding the survey who received oral rehydration salts (ORS)

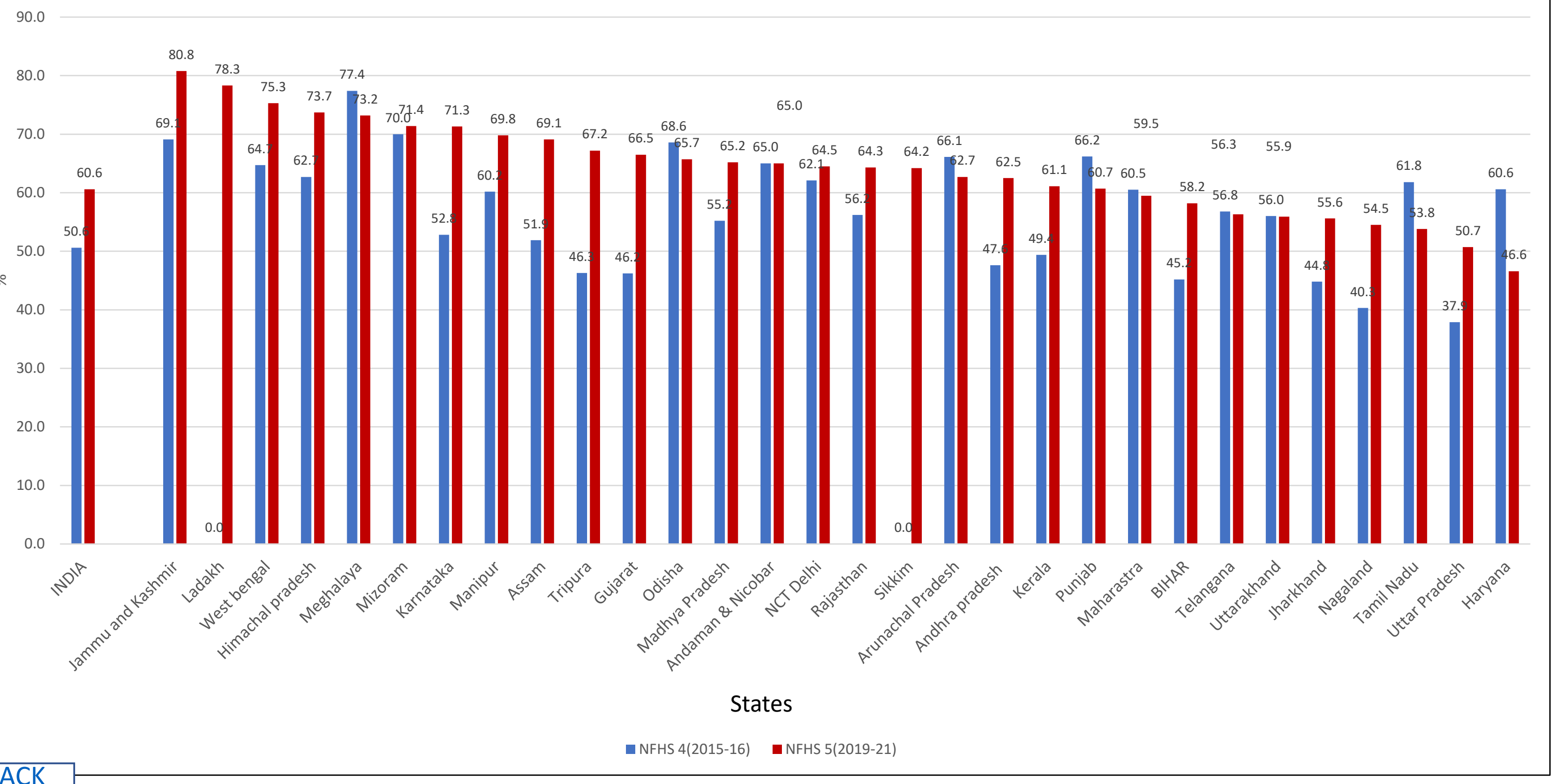




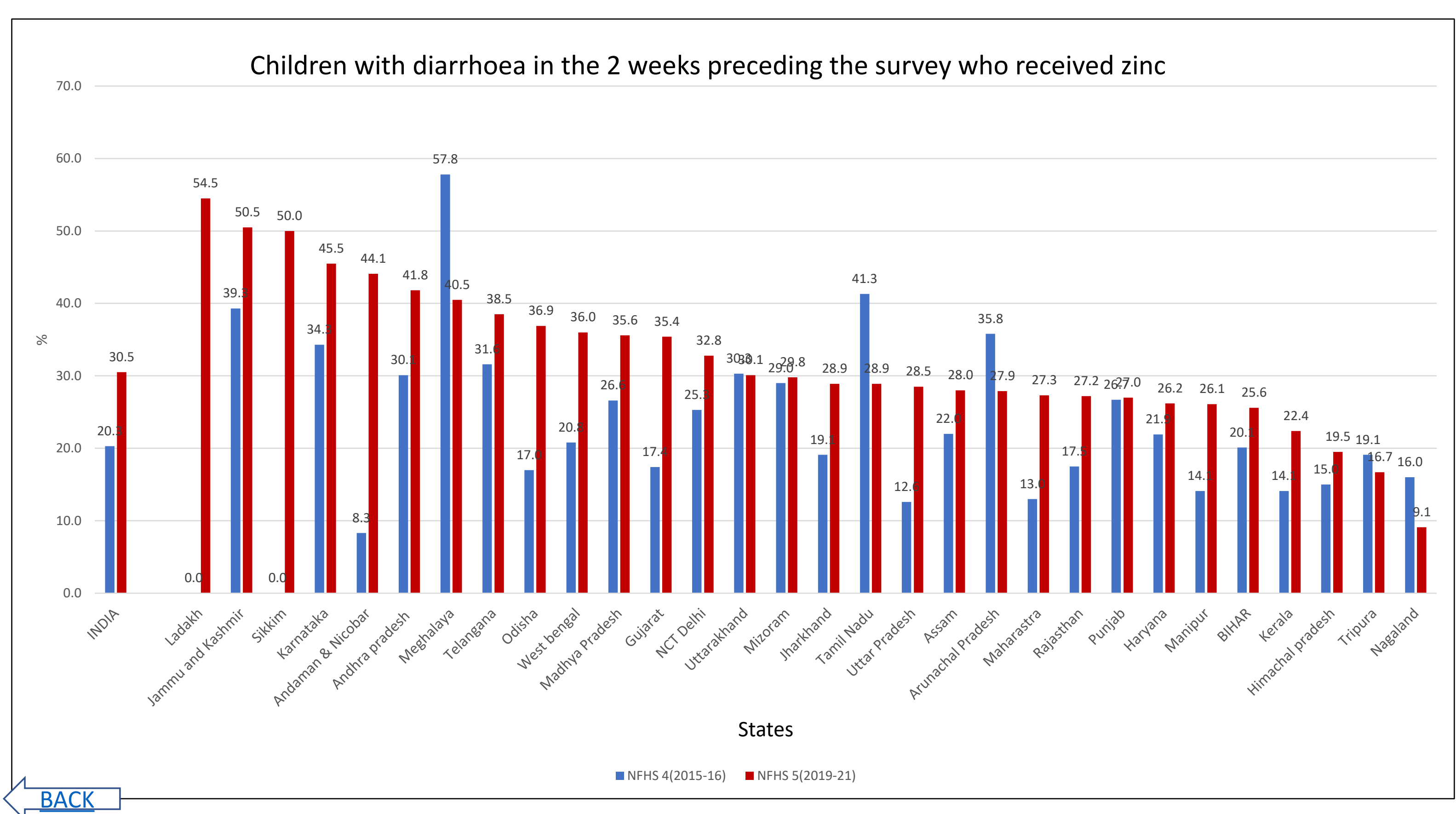


Children under age 3 years breastfed within one hour of birth

90.0

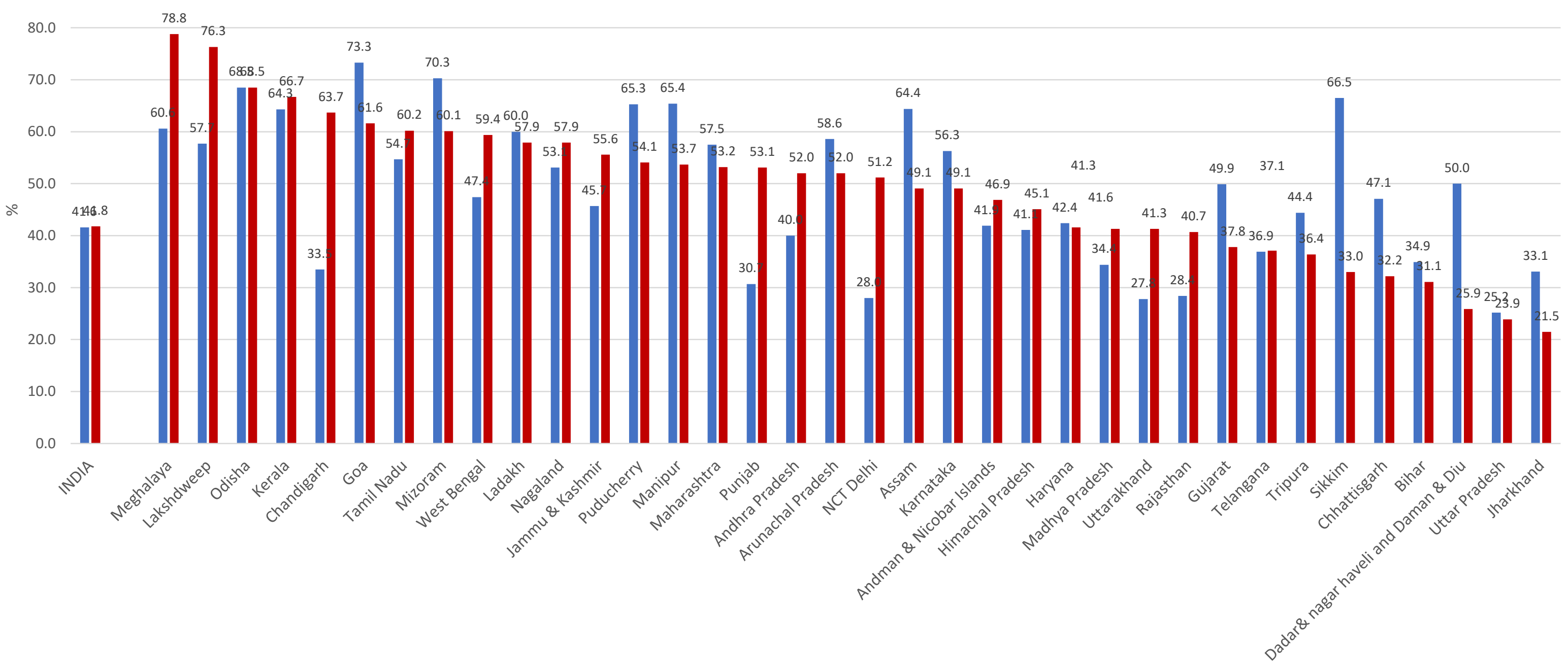

States 
Children under age 6 months exclusively breastfed

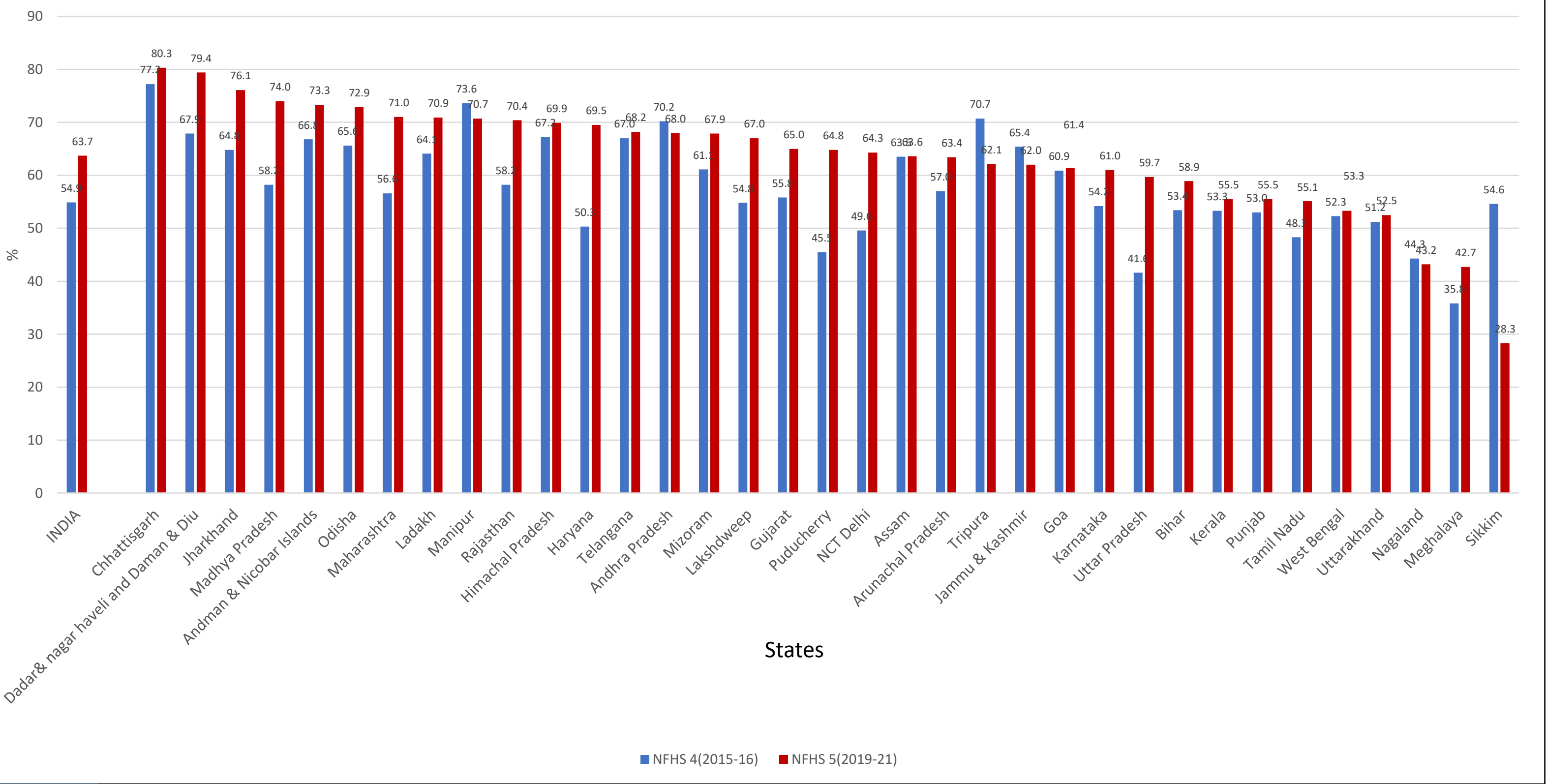




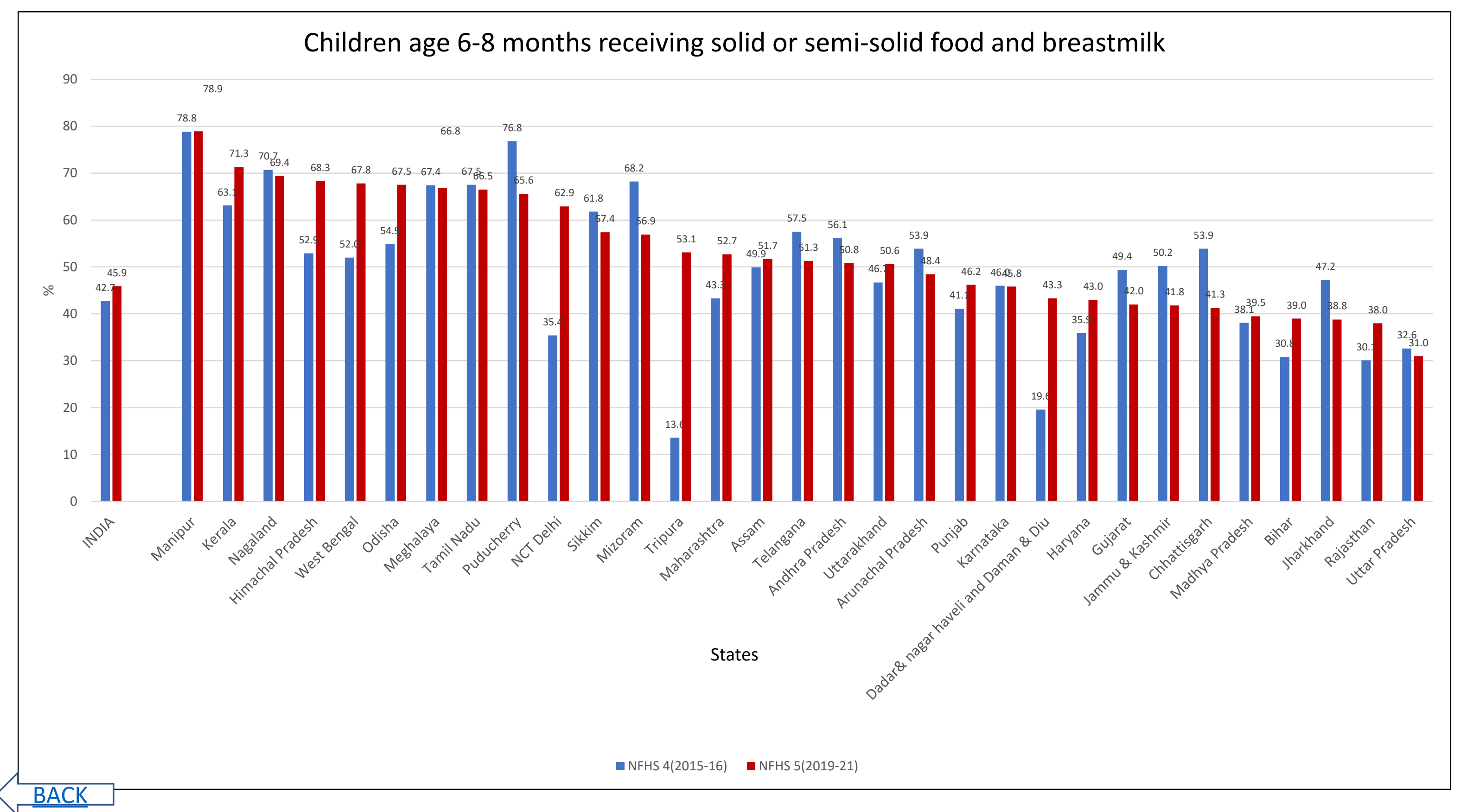




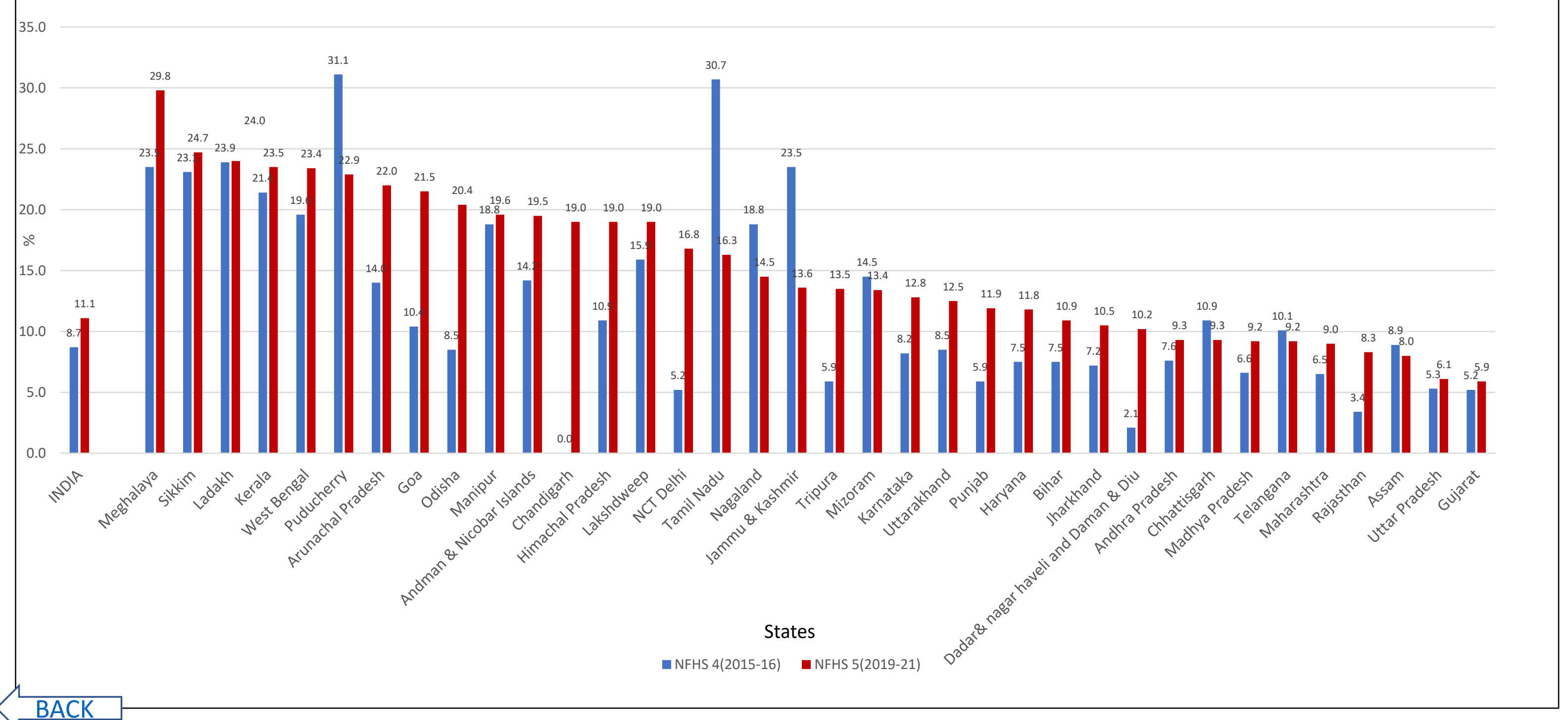


Children age 12-23 months fully vaccinated

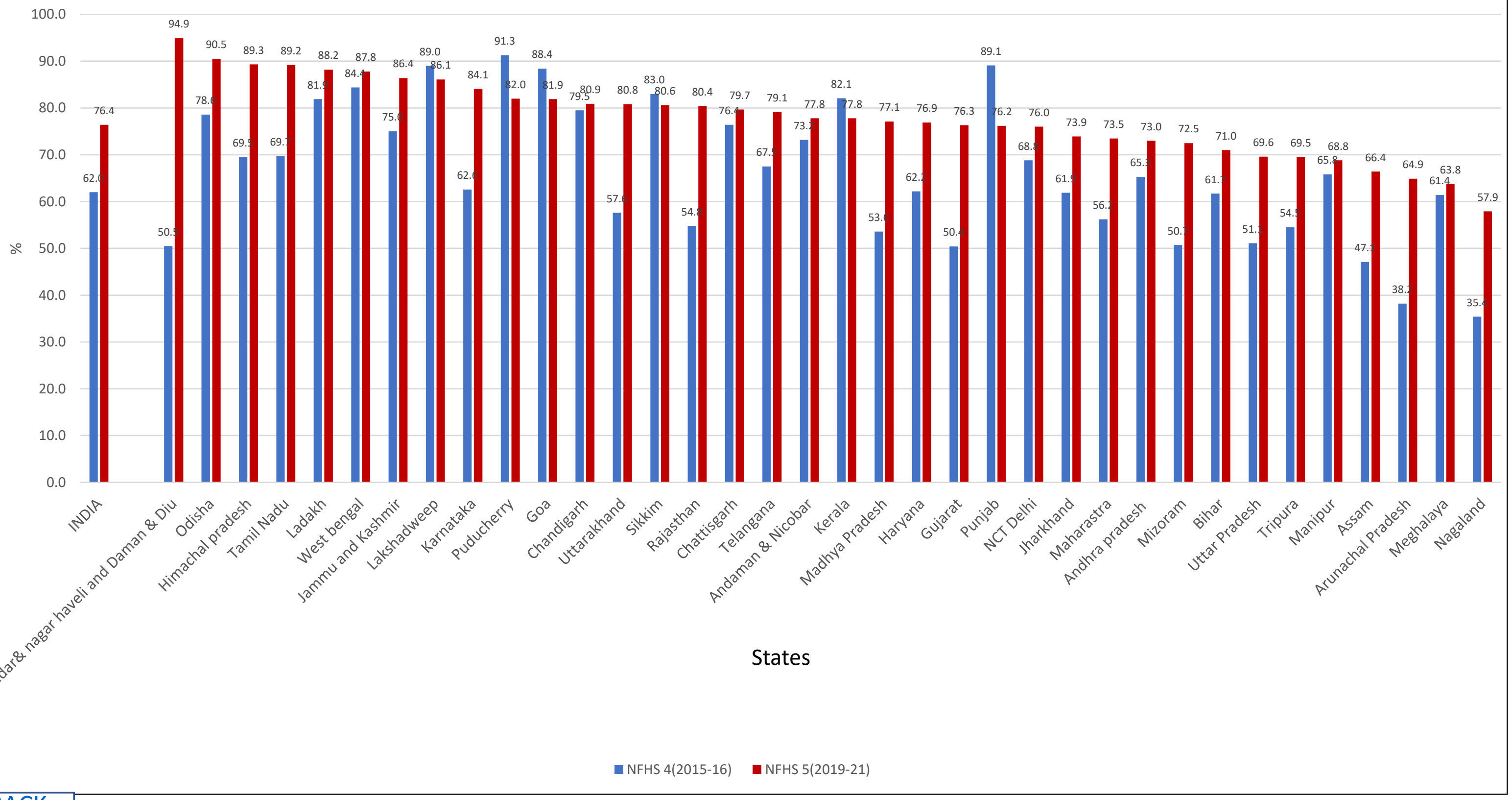




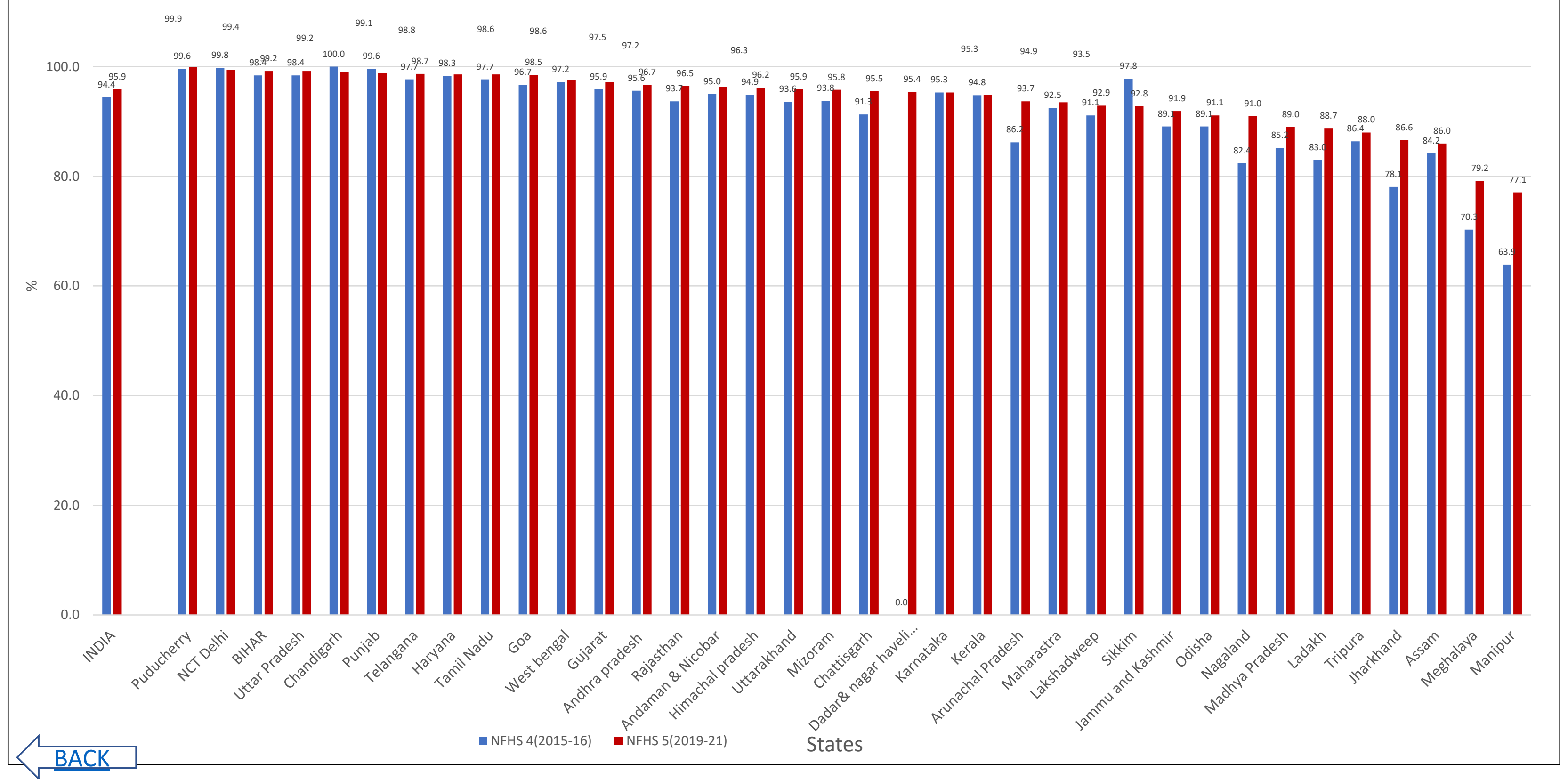


Population living in households with electricity

$99.9 \quad 99.9 \quad 99.8$
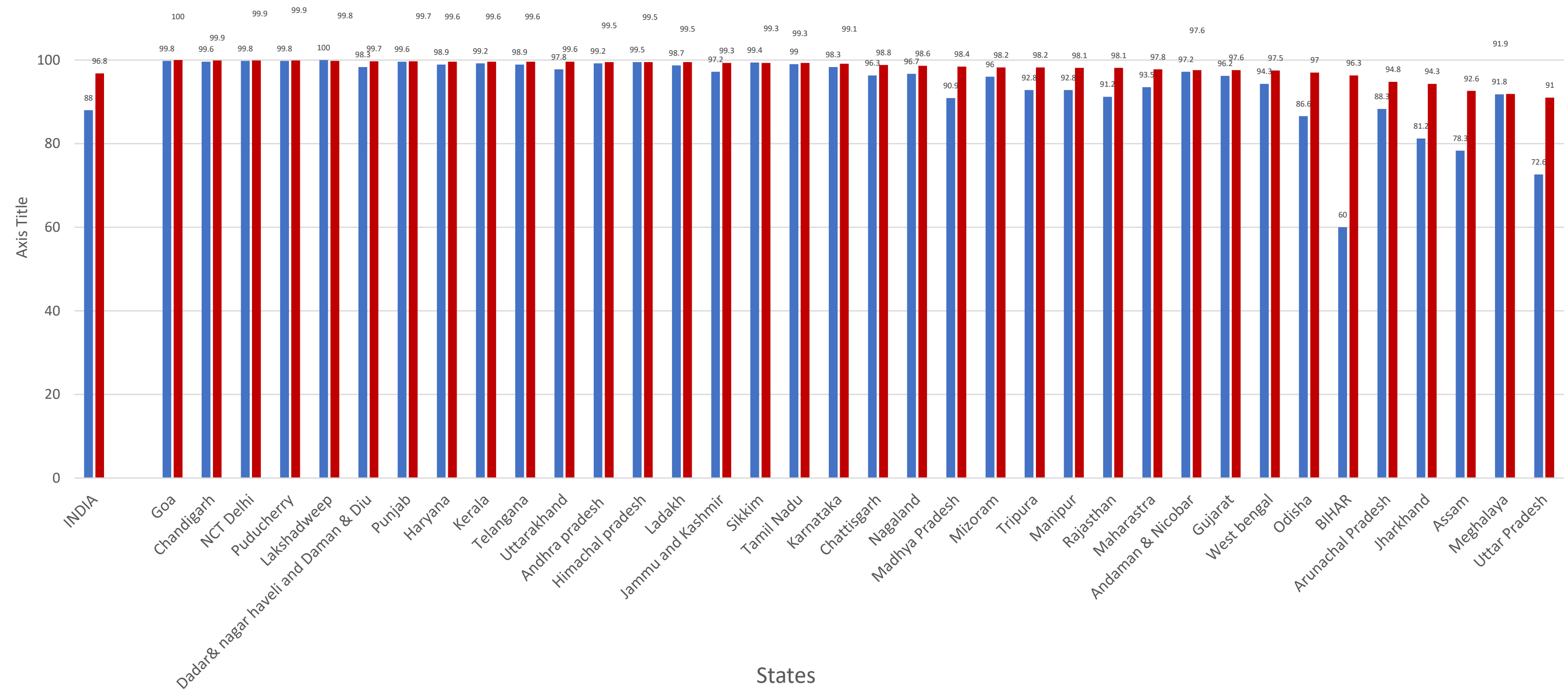


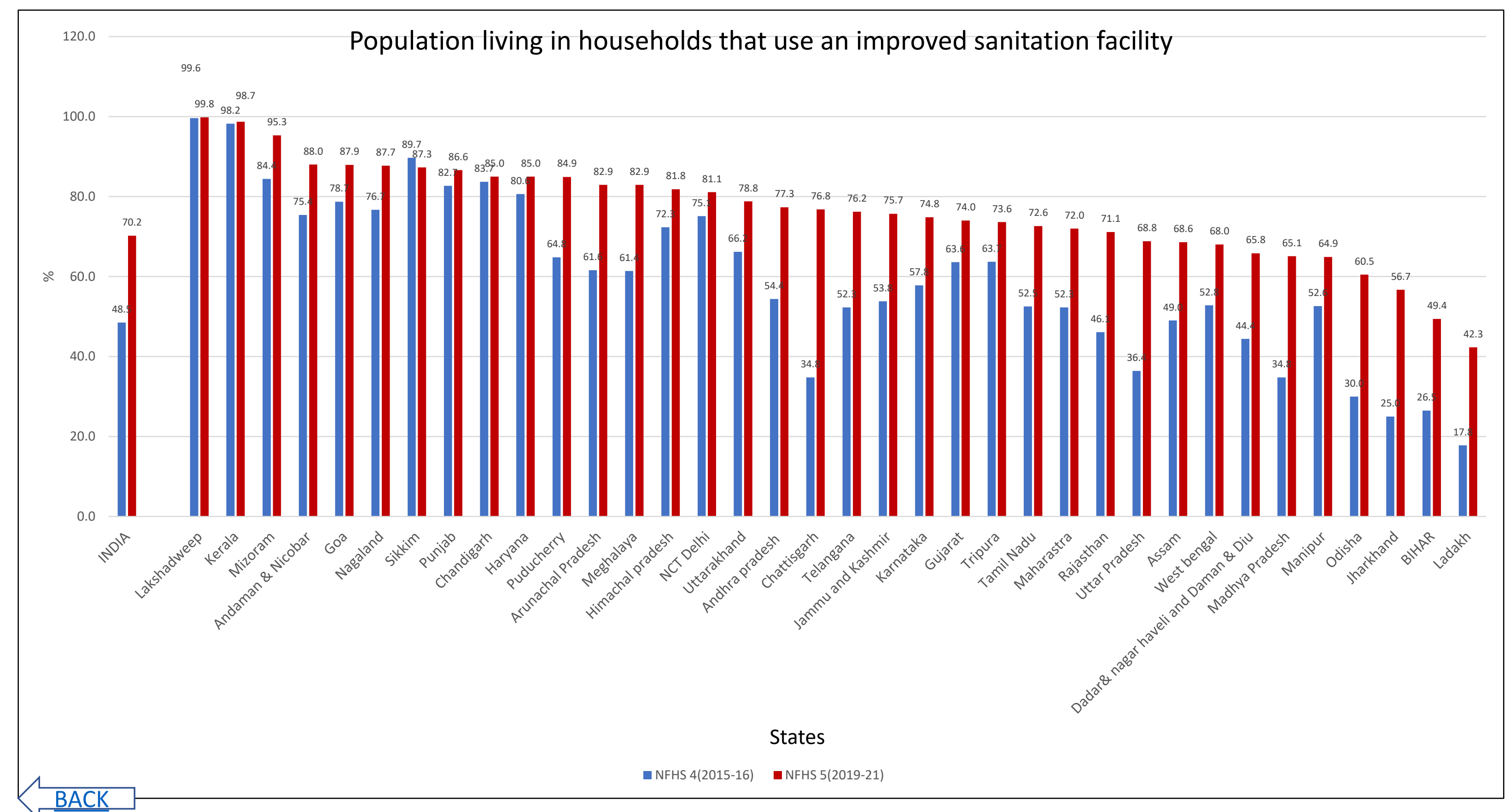


Sex ratio of the total population (females per 1000 males)

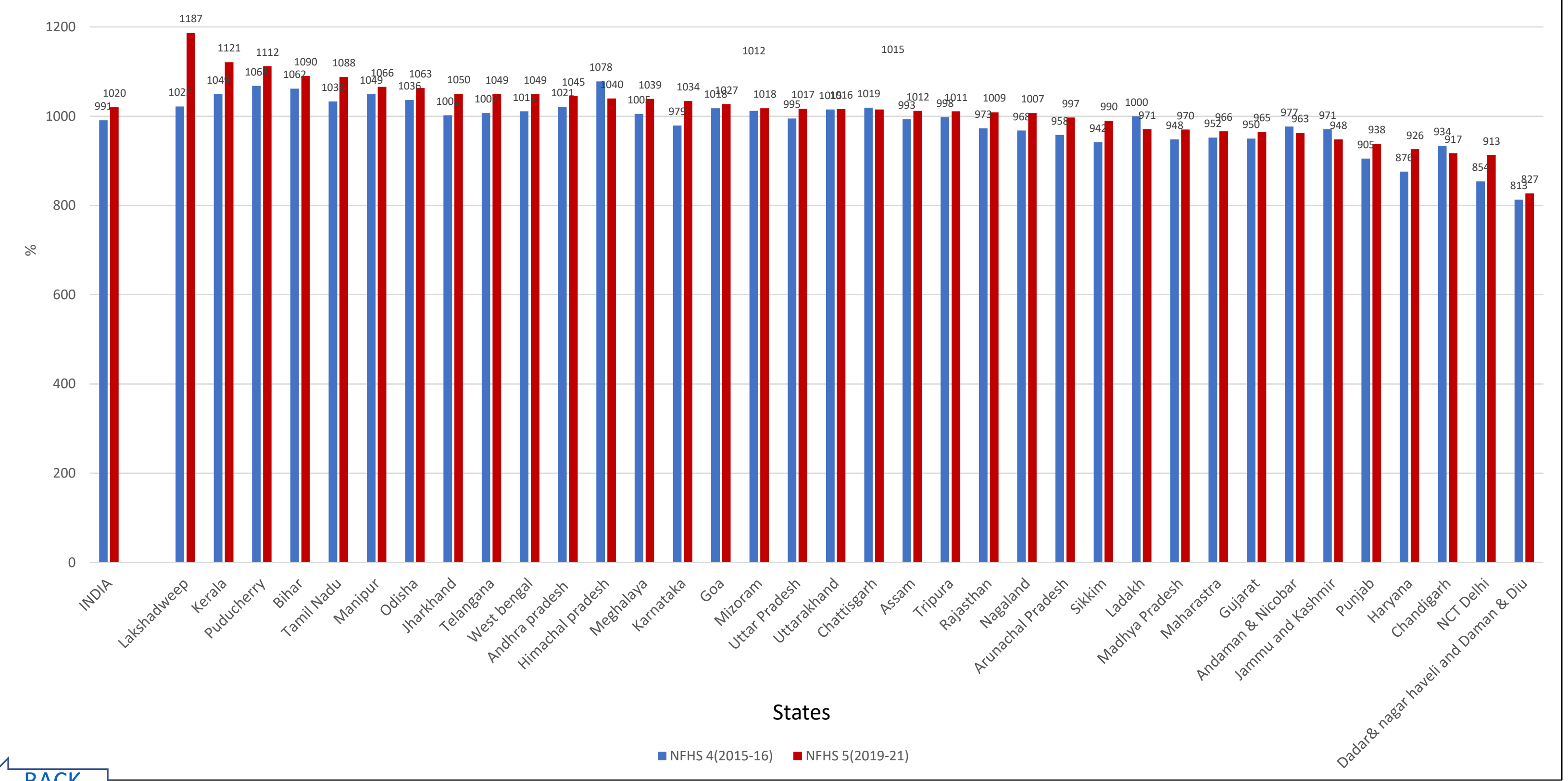


Women with 10 or more years of schooling

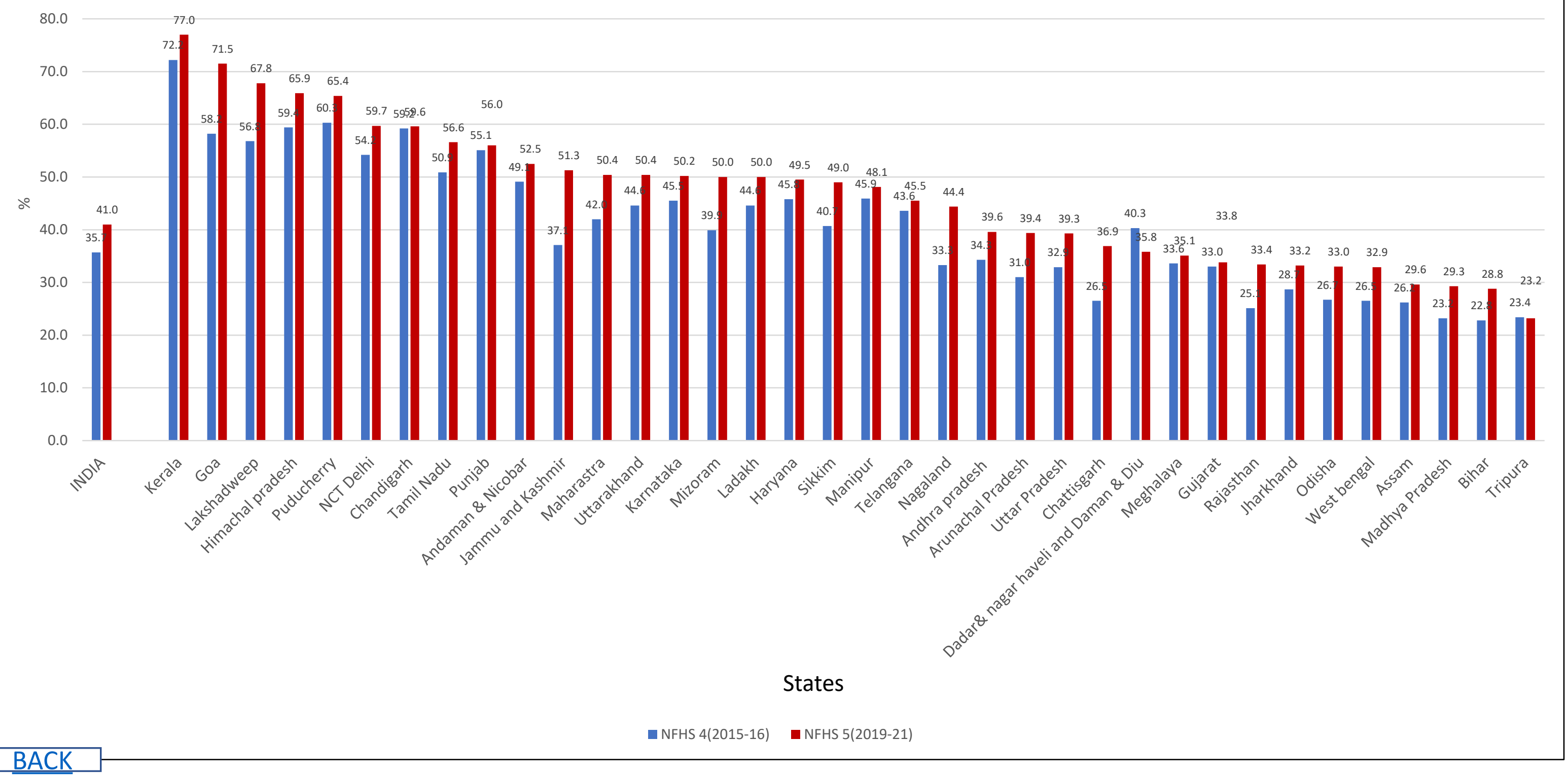


Women age $20-24$ years married before age 18 years

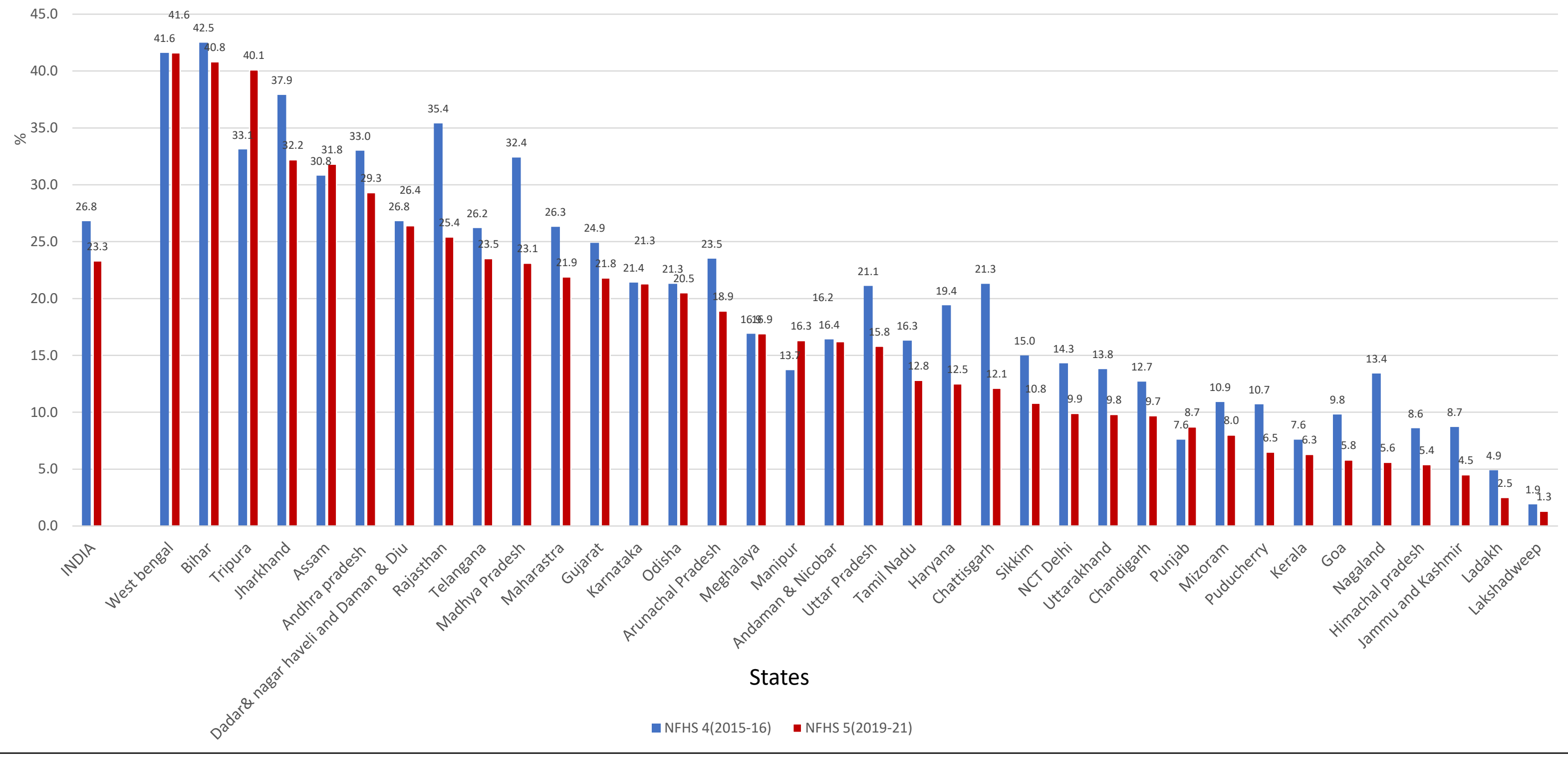




\section{Indicator definitions as per NFHS-5 India factsheet}

\begin{tabular}{|c|c|c|}
\hline S.no & Indicator & Definition \\
\hline 1 & Stunting & $\begin{array}{l}\text { Percentage of children below } 5 \text { yrs of age whose height for age z score is below }-2 \\
\text { standard deviations, based on the WHO standard }\end{array}$ \\
\hline 2 & Wasting & $\begin{array}{l}\text { Percentage of children below } 5 \text { yrs of age whose weight for height z score is below }-2 \\
\text { standard deviations, based on the WHO standard }\end{array}$ \\
\hline 3 & Severe Wasting & $\begin{array}{l}\text { Percentage of children below } 5 \text { yrs of age whose weight for height z score is below }-3 \\
\text { standard deviations, based on the WHO standard. }\end{array}$ \\
\hline 4 & Underweight & $\begin{array}{l}\text { Percentage of children below } 5 \text { yrs of age whose weight for height z score is below }-2 \\
\text { standard deviations, based on the WHO standard }\end{array}$ \\
\hline 5 & Overweight & $\begin{array}{l}\text { Percentage of children below } 5 \text { yrs of age whose weight for height z score is above }+2 \\
\text { standard deviations, based on the WHO standard. }\end{array}$ \\
\hline 6 & Diabetes & $\begin{array}{l}\text { Percentage of adults with random blood sugar level - high or very high }(>140 \mathrm{mg} / \mathrm{dl}) \\
\text { or taking medicine to control blood sugar level }\end{array}$ \\
\hline 7 & Hypertension & $\begin{array}{l}\text { Percenatge of adults with elevated blood pressure (Systolic } \geq 140 \mathrm{~mm} \text { of } \mathrm{Hg} \text { and/or } \\
\text { Diastolic } \geq 90 \mathrm{~mm} \text { of } \mathrm{Hg} \text { ) or taking medicine to control blood pressure }\end{array}$ \\
\hline
\end{tabular}




\begin{tabular}{|c|c|c|}
\hline S.no & Indicator & Definition \\
\hline 10 & Underweight among women & $\begin{array}{l}\text { Percentage of women whose Body Mass Index }(\mathrm{BMI}) \text { is below normal }(<18.5 \mathrm{~kg} / \mathrm{m} 2) \text {. } \\
\text { Excludes pregnant women and women with a birth in the preceding } 2 \text { months. }\end{array}$ \\
\hline 11 & Underweight among men & Percentage of men whose Body Mass Index (BMI) is below normal $(<18.5 \mathrm{~kg} / \mathrm{m} 2)$ \\
\hline 12 & Overweight among women & $\begin{array}{l}\text { Percentage of women who are overweight or obese (BMI } \geq 25.0 \mathrm{~kg} / \mathrm{m} 2) \text {. Excludes } \\
\text { pregnant women and women with a birth in the preceding } 2 \text { months }\end{array}$ \\
\hline 13 & Overweight among men & Men who are overweight or obese (BMI $\geq 25.0 \mathrm{~kg} / \mathrm{m} 2$ ) \\
\hline 14 & Anemia in children (6-59 months) & $\begin{array}{l}\text { Percentage of children age } 6-59 \text { months who are anaemic }(<11.0 \mathrm{~g} / \mathrm{dL}) \text {. Among children, } \\
\text { prevalence is adjusted for altitude }\end{array}$ \\
\hline 15 & Anemia in adolescent girls & All women age $15-19$ years who are anaemic \\
\hline 16 & Anemia in adolescent boys & All men age $15-19$ years who are anaemic $(<13.0 \mathrm{~g} / \mathrm{dL})$. \\
\hline 17 & Anemia in pregnant women & $\begin{array}{l}\text { Percentage of pregnant women age } 15-49 \text { years who are anaemic }(<11.0 \mathrm{~g} / \mathrm{dL}) \\
\text { Among adults, prevalence is adjusted for altitude and for smoking status, if known }\end{array}$ \\
\hline 18 & Anemia in all men & $\begin{array}{l}\text { Percentage of all Men age } 15-49 \text { years who are anaemic }(<13.0 \mathrm{~g} / \mathrm{dL}) \text {. Among adults, } \\
\text { prevalence is adjusted for altitude and for smoking status, if known }\end{array}$ \\
\hline 19 & Anemia in all women & $\begin{array}{l}\text { Percentage of all women age } 15-49 \text { years who are anaemic }(<12.0 \mathrm{~g} / \mathrm{dL}) \text {. Among adults, } \\
\text { prevalence is adjusted for altitude and for smoking status, if known }\end{array}$ \\
\hline
\end{tabular}




\section{S.no} Indicator

Iron Folic acid supplement for $100+$ days

21 Iron Folic acid supplement for 180 days

22 Vitamin A supplementation among children

23 ANC check-up in first trimester

$24 \quad$ Mothers who received PNC

25 Prevalence of Diarrhoea

26 Prevalence of ARI

$27 \quad$ Full Vaccination

$28 \quad$ Exclusive Breastfeeding

Introduction of semisolids

29 NEXT
Definition

Percentage of mothers who consumed iron folic acid for 100 days or more when they were pregnant

Percentage of mothers who consumed iron folic acid for 180 days or more when they were pregnant

Percentage of children age 9-35 months who received a vitamin A dose in the last 6 months

Percentage of mothers who had an antenatal check-up in the first trimester

Percentage of mothers who received postnatal care from a doctor/nurse/LHV/ANM/midwife/other health personnel within 2 days of delivery

Percentage of children less than 5 yrs of age who had diarrhoea in the 2 weeks preceding the survey

Percentage of children less than 5 yrs of age who had symptoms of acute respiratory infection (ARI) in the 2 weeks preceding the survey

Percentage of children age 12-23 months fully vaccinated (Vaccinated with BCG, measlescontaining vaccine (MCV)/MR/MMR/Measles, and 3 doses each of polio (excluding polio vaccine given at birth) and DPT or penta vaccine) based on information from either vaccination card or mother's recall.

Percentage of youngest children, living with mother, under age 6 months exclusively breastfed

Percentage of youngest children age 6-8 months, living with mother, receiving solid or semisolid food and breastmilk 


\begin{tabular}{|c|c|c|}
\hline S.no & Indicator & Definition \\
\hline 30 & $\begin{array}{l}\text { Adequate diet among } \\
\text { children }\end{array}$ & $\begin{array}{l}\text { Percentage of total children age 6-23 months receiving an adequate diet. Breastfed children receiving } 4 \\
\text { or more food groups and a minimum meal frequency, non-breastfed children fed with a minimum of } 3 \\
\text { Infant and Young Child Feeding Practices (fed with other milk or milk products at least twice a day, a } \\
\text { minimum meal frequency that is, receiving solid or semi-solid food at least twice a day for breastfed } \\
\text { infants 6-8 months and at least three times a day for breastfed children } 9-23 \text { months, and solid or semi- } \\
\text { solid foods from at least four food groups not including the milk or milk products food group). }\end{array}$ \\
\hline 31 & $\begin{array}{l}\text { Early initiation of } \\
\text { Breastfeeding }\end{array}$ & $\begin{array}{l}\text { Percentage of children under age } 3 \text { years breastfed within one hour of birth for the last child born in the } \\
3 \text { years before the survey. }\end{array}$ \\
\hline 32 & $\begin{array}{l}\text { HHs with improved } \\
\text { drinking water }\end{array}$ & $\begin{array}{l}\text { Percentage of population living in households with an improved drinking-water source - having piped } \\
\text { water into dwelling/yard/plot, piped to neighbour, public tap/standpipe, tube well or borehole, } \\
\text { protected dug well, protected spring, rainwater, tanker truck, cart with small tank, bottled water, } \\
\text { community RO plant. }\end{array}$ \\
\hline 33 & HHs with electricity & Percentage of Population living in households with electricity \\
\hline 34 & $\begin{array}{l}\text { HHs with improved } \\
\text { sanitation facility }\end{array}$ & $\begin{array}{l}\text { Percentage of Population living in households that use an improved sanitation facility - Flush to piped } \\
\text { sewer system, flush to septic tank, flush to pit latrine, flush to don't know where, ventilated improved } \\
\text { pit (VIP)/biogas latrine, pit latrine with slab, twin pit/composting toilet, which is not shared with any } \\
\text { other household. This indicator does not denote access to toilet facility }\end{array}$ \\
\hline 35 & $\begin{array}{l}\text { Women with } 10 \text { or more } \\
\text { years of schooling }\end{array}$ & Percentage of Women with 10 or more years of schooling \\
\hline 36 & $\begin{array}{l}\text { Women married before } 18 \\
\text { years }\end{array}$ & Percentage of women age $20-24$ years married before age 18 years \\
\hline
\end{tabular}


Thank you 